FINITE ELEMENT EXTERIOR CALCULUS, HOMOLOGICAL TECHNIQUES, AND APPLICATIONS

By

Douglas N. Arnold

Richard S. Falk

and

Ragnar Winther

IMA Preprint Series \# 2094

( February 2006)

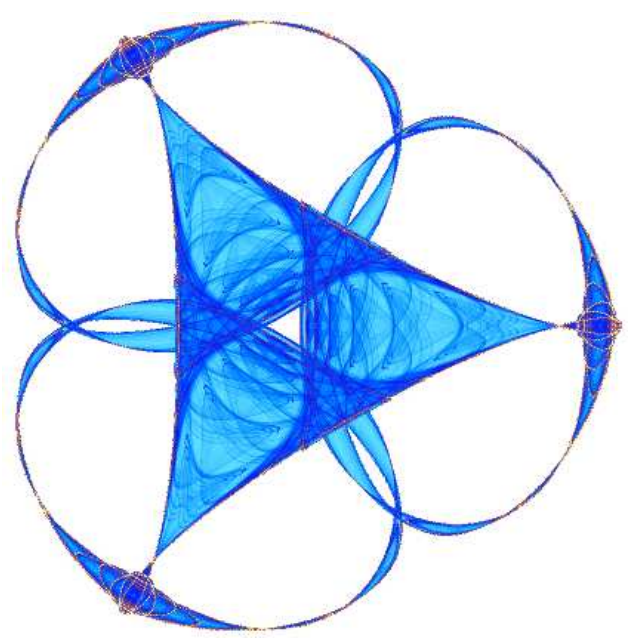

INSTITUTE FOR MATHEMATICS AND ITS APPLICATIONS

UNIVERSITY OF MINNESOTA 400 Lind Hall

207 Church Street S.E.

Minneapolis, Minnesota 55455-0436

Phone: 612/624-6066 Fax: 612/626-7370

URL: http://www.ima.umn.edu 


\title{
Finite element exterior calculus, homological techniques, and applications
}

\author{
Douglas N. Arnold \\ Institute for Mathematics and its Applications \\ and School of Mathematics, \\ University of Minnesota, Minneapolis, MN 55455, USA \\ E-mail: arnold@ima.umn.edu \\ Richard S. Falk \\ Department of Mathematics, \\ Rutgers University, Piscataway, NJ 08854, USA \\ E-mail: falk@math.rutgers.edu \\ Ragnar Winther \\ Centre of Mathematics for Applications \\ and Department of Informatics, \\ University of Oslo, 0316 Oslo, Norway \\ E-mail: ragnar.winther@cma.uio.no \\ Dedicated to Carme, Rena, and Rita.
}

Finite element exterior calculus is an approach to the design and understanding of finite element discretizations for a wide variety of systems of partial differential equations. This approach brings to bear tools from differential geometry, algebraic topology, and homological algebra to develop discretizations which are compatible with the geometric, topological, and algebraic structures which underlie well-posedness of the PDE problem being solved. In the finite element exterior calculus, many finite element spaces are revealed as spaces of piecewise polynomial differential forms. These connect to each other in discrete subcomplexes of elliptic differential complexes, and are also related to the continuous elliptic complex through projections which commute with the complex differential. Applications are made to the finite element discretization of a variety of problems, including the Hodge Laplacian, Maxwell's equations, the equations of elasticity, and elliptic eigenvalue problems, and also to preconditioners. 


\section{CONTENTS}

1 Introduction 2

Part 1: Exterior calculus, finite elements, and homology

2 Exterior algebra and exterior calculus 8

3 Polynomial differential forms and the Koszul complex 27

4 Polynomial differential forms on a simplex 41

5 Finite element differential forms and their cohomology 58

6 Differential forms with values in a vector space $\quad 74$

Part 2: Applications to discretization of differential equations

7 The Hodge Laplacian $\quad 78$

8 Eigenvalue problems $\quad 94$

9 The $H \Lambda$ projection and Maxwell's equations 101

10 Preconditioning $\quad 106$

11 The elasticity equations 121

$\begin{array}{ll}\text { References } & 149\end{array}$

\section{Introduction}

The finite element method is one of the greatest advances in numerical computing of the past century. It has become an indispensable tool for simulation of a wide variety of phenomena arising in science and engineering. A tremendous asset of finite elements is that they not only provide a methodology to develop numerical algorithms for simulation, but also a theoretical framework in which to assess the accuracy of the computed solutions. In this paper we survey and develop the finite element exterior calculus, a new theoretical approach to the design and understanding of finite element discretizations for a wide variety of systems of partial differential equations. This approach brings to bear tools from differential geometry, algebraic topology, and homological algebra to develop discretizations which are compatible with the geometric, topological, and algebraic structures which underlie well-posedness of the PDE problem being solved. Applications treated here include the finite element discretization of the Hodge Laplacian (which includes many problems as particular cases), Maxwell's equations of electromagnetism, the equations of elasticity, and elliptic eigenvalue problems, and the construction of preconditioners.

To design a finite element method to solve a problem in partial differential equations, the problem is first given a weak or variational formulation which characterizes the solution among all elements of a given space of functions on the domain of interest. A finite element method for this problem proceeds with the construction of a finite-dimensional subspace of the given 
function space where the solution is sought, and then the specification of a unique element of this subspace as the solution of an appropriate set of equations on this finite-dimensional space. In finite element methods, the subspace is constructed from a triangulation or simplicial decomposition of the given domain, using spaces of polynomials on each simplex, pieced together by a certain assembly process. Because the finite element space so constructed is a subspace of the space where the exact solution is sought, one can consider the difference between the exact and finite element solution and measure it via appropriate norms, seminorms, or functionals. Generally speaking, error bounds can be obtained in terms of three quantities: the approximation error, which measures the error in the best approximation of the exact solution possible within the finite element space, the consistency error, which measures the extent to which the equations used to select the finite element solution from the finite element space reflect the continuous problem, and the stability constant, which measures the well-posedness of the finite-dimensional problem. The approximation properties of finite element spaces are well understood, and the consistency of finite element methods is usually easy to control (in fact, for all the methods considered in this paper there is no consistency error in the sense that the exact solution will satisfy the natural extension of the finite element equations to solution space). In marked contrast, the stability of finite element procedures can be very subtle. For many important problems, the development of stable finite element methods remains extremely challenging or even out of reach, and in other cases it is difficult to assess the stability of methods of interest. Lack of stable methods not only puts some important problems beyond the reach of simulation, but has also led to spectacular and costly failures of numerical methods.

It should not be surprising that stability is a subtle matter. Establishing stability means proving the well-posedness of the discrete equations, uniformly in the discretization parameters. Proving the well-posedness of PDE problems is, of course, the central problem of the theory of PDEs. While there are PDE problems for which this is a simple matter, for many important problems it lies deep, and a great deal of mathematics, including analysis, geometry, topology, and algebra, has been developed to establish the well-posedness of various PDE problems. So it is to be expected that a great deal of mathematics bears as well on the stability of numerical methods for PDE. An important but insufficiently appreciated point is that approximability and consistency together with well-posedness of the continuous problem do not imply stability. For example, one may consider a PDE problem whose solution is characterized by a variational principle, i.e., as the unique critical point of some functional on some function space, and define a finite element method by seeking a critical point of the same functional (so there is no consistency error) on a highly accurate finite el- 
ement space (based on small elements and/or high-order polynomials, so there is arbitrary low approximability error), and yet such a method will very often be unstable and therefore not convergent. Analogously, in the finite difference methodology, one may start with a PDE problem stated in strong form and replace the derivatives in the equation by consistent finite differences, and yet obtain a finite difference method which is unstable.

As mentioned, the well-posedness of many PDE problems reflects geometrical, algebraic, topological, and homological structures underlying the problem, formalized by exterior calculus, Hodge theory, and homological algebra. In recent years there has been a growing realization that stability of numerical methods can be obtained by designing methods which are compatible with these structures in the sense that they reproduce or mimic them (not just approximate them) on the discrete level. See, for example, Arnold (2002), and the volume edited by Arnold, Bochev, Lehoucq, Nicolaides and Shashkov $(2006 a)$. In the present paper, the compatibility is mostly related to elliptic complexes which are associated with the PDEs under consideration, mostly the de Rham complex and its variants and another complex associated with the equations of linear elasticity. Our finite element spaces will arise as the spaces in finite-dimensional subcomplexes which inherit the cohomology and other features of the exact complexes. The inheritance will generally be established by cochain projections: projection operators from the infinite-dimensional spaces in the original elliptic complex which map onto the finite element subspaces and commute with the differential operators of the complex. Thus the main theme of the paper is the development of finite element subcomplexes of certain elliptic differential complexes and cochain projections onto them, and their implications and applications in numerical PDEs. We refer to this theme and the mathematical framework we construct to carry it out, as finite element exterior calculus.

We mention some of the computational challenges which motivated the development of finite element exterior calculus and which it has helped to address successfully. These are challenges both of understanding the poor behaviour of seemingly reasonable numerical methods, and of developing effective methods. In each case, the finite element exterior calculus provides an explanation for the difficulties experienced with naive methods, and also points to a practical finite element solution.

- The system $\sigma=\operatorname{grad} u, \operatorname{div} u=f$ (the Poisson equation written in first-order form) is among the simplest and most basic PDEs. But even in one dimension, the stability of finite element discretizations for it are hard to predict. For example, the use of classical continuous piecewise linear elements for both $\sigma$ and $u$ is unstable. See Arnold, Falk and Winther (2006b) for a discussion and numerical examples. This is one of many problems handled in a unified manner by the finite 
element exterior calculus approach to the Hodge Laplacian given in Section 7 .

- A standard variational form for the vector Poisson problem is to minimize the energy $\int\left[\left(|\operatorname{div} u|^{2}+|\operatorname{curl} u|^{2}\right) / 2-f u\right] \mathrm{d} x$ over vector fields satisfying the boundary condition, say, $u \cdot n=0$ on the boundary. This problem is well-posed, but a standard finite element method which proceeds by minimizing the energy over a piecewise polynomial subspace of the energy space, will inevitably converge to the wrong solution on some domains, e.g., nonconvex polygons or polyhedra. See the end of Section 2.2 for more on this.

- With naive choices of finite element spaces, the computed spectrums of some elliptic eigenvalue problems related to the vector Laplacian or Maxwell's equation, bear no relation to the true spectrum, or include spurious eigenvalues that converge to a point outside the true spectrum as the mesh is refined. See, e.g., Boffi (2006) for more on this and numerical examples. Application of the finite element exterior calculus makes the computation and numerical analysis of such eigenvalue problems straightforward, as explained in Section 8.

- The equations of elasticity are classically among the most important applications of the finite element method. A natural variational formulation for them is the Hellinger-Reissner principle. Despite four decades of searching, stable finite elements for this variational principle have proved elusive. The first such using polynomial shape functions were presented in Arnold and Winther (2002) for plane elasticity, using the ideas of finite element exterior calculus and spurring further development. New families of stable elements for elasticity in arbitrary dimensions are presented in Section 11.

- Multigrid methods based on the additive Schwartz smoothers used for standard finite element methods do not work when applied to some other problems involving the $H$ (curl)-inner product. But a different choice of the additive Schwartz smoother does lead to effective multigrid solvers. See Section 10.

The finite element exterior calculus is not the only compatible discretization approach in active development. It certainly has motivations and features in common with mimetic finite difference methods (Bochev and Hyman 2006), covolume methods (Nicolaides and Trapp 2006), and the discrete exterior calculus (Desbrun, Hirani, Leok and Marsden 2005). Moreover, there are coincidences of methods in simple cases. However, there are also major differences. In mimetic finite differences and the discrete exterior calculus, the fundamental object is a simplicial cochain, a function assigning a number to each simplex of dimension $k$ of the simplicial complex. There is, of course, a close relation between differential forms and 
simplicial cochains. A differential form of degree $k$ can be integrated over a simplex of dimension $k$ to give a number, and de Rham's theorem states that this map from the de Rham complex to the simplicial cochain complex induces an isomorphism on cohomology. It is less obvious how to go from a cochain to a differential form. An answer was provided by Whitney, who constructed a one-sided inverse of the de Rham map by associating to any $k$-cochain a piecewise linear differential $k$-form. Bossavit (1988) recognized that the Whitney forms coincided with low-order finite element spaces that had been developed for electromagnetism. In view of all this, there is a very close relationship between mimetic finite differences and discrete exterior calculus, on the one hand, and the Whitney form complex of the finite element exterior calculus. But the finite element exterior calculus described in this paper involves much more than the Whitney forms, namely two families of finite element spaces of differential forms, $\mathcal{P}_{r} \Lambda^{k}\left(\mathcal{T}_{h}\right)$ and $\mathcal{P}_{r}^{-} \Lambda^{k}\left(\mathcal{T}_{h}\right)$, with a rich structure of interconnections between them. Of these, the spaces $\mathcal{P}_{1}^{-} \Lambda^{k}\left(\mathcal{T}_{h}\right)$ are the Whitney forms and isomorphic to simplicial cochains, while the others do not naturally fit in the simplicial cochain formalism. As pointed out at the start of this Introduction, a great strength of finite element methods is that the discrete solution belongs to the same function space as the exact solution and so comparison is natural. For this reason we view the realization of finite elements as differential forms, rather than as discrete objects which mimic differential forms, as highly desirable.

Much of the foundation of finite element exterior calculus was developed by many people over a long period of time. Besides the work of Whitney and Bossavit already mentioned, we signal the contributions of Hiptmair (Hiptmair 1999a, Hiptmair 2001, Hiptmair 2002), especially to the aspects of the theory that are relevant for electromagnetic problems. Interest in the subject grew with the 2002 presentation at the International Congress of Mathematicians (Arnold 2002) where the strong connection between numerical stability and cochain projections of elliptic complexes was first emphasized. Many other references will be made throughout the paper, but the literature is too large to reference anywhere near all the relevant work.

This paper is more than a survey of an existing theory. We also present a number of results here which either are new, or appeared only recently in our other work. For example, we emphasize the relevance of the Koszul complex in constructing finite element subspaces of spaces of differential forms and determining their properties. The Koszul differential leads to the family of finite element spaces $\mathcal{P}_{r}^{-} \Lambda^{k}\left(\mathcal{T}_{h}\right)$, including the Whitney forms $(r=1)$.

The two families of spaces $\mathcal{P}_{r} \Lambda^{k}\left(\mathcal{T}_{h}\right)$ and $\mathcal{P}_{r}^{-} \Lambda^{k}\left(\mathcal{T}_{h}\right)$ include among them, for special values of $r$ and $k$, the Lagrange finite elements and most of the stable finite element spaces that have been used to solve mixed formulations of the Poisson or Maxwell's equations. A new aspect of this paper is the 
development of bases and degrees of freedom for all these spaces in a unified fashion that is not possible when the spaces are studied in isolation from each other. In particular, the degrees of freedom we present are in some cases more natural than the ones that have appeared previously in the literature.

As alluded to, a key feature of our approach is the arrangement of these spaces of finite element differential forms into finite dimensional subcomplexes of the de Rham complex. In fact, we show that for each polynomial degree there are $2^{n-1}$ such subcomplexes in $n$ dimensions which reproduce the same cohomology. Some of these complexes had been studied before, especially the celebrated complex of Whitney forms and its higher degree generalizations, but many are new. Some of these new complexes turn out to be essential for the discretization of the elasticity system.

Another direction which is new to this paper is the use of group representation theory to characterize the spaces of polynomial differential forms $\mathcal{P}_{r} \Lambda^{k}\left(\mathbb{R}^{n}\right)$ and $\mathcal{P}_{r}^{-} \Lambda^{k}\left(\mathbb{R}^{n}\right)$ which are used to construct the finite element differential forms. In Section 3.4, we show that these spaces are nearly the only ones with a certain affine invariance property.

The development of finite element methods with weakly imposed symmetry for the elasticity equations as presented here appeared only recently in our work. A key tool, not previously used in numerical analysis, is the BGG resolution. The presentation here is more general and simpler than the previous versions, and the treatment of traction boundary conditions first appears here.

A great strength of the exterior calculus approach is the way it unifies seemingly different problems in a common framework. A familiar example is the unification of the three main first-order differential operators of vector calculus, grad, div, and curl, as a single operator, the exterior derivative, d. In a similar way, the Hodge Laplacian, $\mathrm{d} \delta+\delta \mathrm{d}$, where $\delta$ is the formal adjoint of $d$, unifies many important second-order differential operators. By studying the numerical solution of source and eigenvalue properties and preconditioners for the Hodge Laplacian in generality, we simultaneously treat many different problems, including the standard finite element methods with Lagrange elements for the scalar Laplacian, mixed finite elements for the Laplacian, different mixed finite element formulations for the vector Laplacian, curl curl problems arising in electromagnetics in different formulations, and div-curl systems.

We also mention some more technical aspects of the current presentation that distinguish it from others that appear in the literature. We treat domains of full topological generality, that is, with arbitrary Betti numbers. Thus our goal is not to show that the finite element de Rham subcomplexes we construct are exact, but rather that they reproduce the de Rham cohomology of the domain. When we consider boundary value problems for the Hodge Laplacian, we must take into account the harmonic forms, and 
so well-posedness depends on a finite number of compatibility conditions of the data and uniqueness requires a finite number of side conditions, and we reproduce this situation on the discrete level.

Another technical aspect of the presentation is that we use not only the canonical commuting projection operators into the finite element spaces, but also smoothed variants of these, which still commute with the exterior derivative and which have better boundedness properties. These allow simpler and cleaner analysis in many places. The construction of such bounded cochain projections is a recent and active subject of research. We present an approach here inspired by Schöberl (2005) and Christiansen (2005), while recognizing that it is very possibly not the last word in this matter.

The contents of the paper are as follows. In the first part of the paper, we develop the finite element exterior calculus, starting with a review of the necessary exterior algebra, exterior calculus, and Hodge theory. We then turn to polynomial differential forms which are intimately related to the Koszul complex. Here we develop the polynomial spaces $\mathcal{P}_{r} \Lambda^{k}\left(\mathbb{R}^{n}\right)$ and $\mathcal{P}_{r}^{-} \Lambda^{k}\left(\mathbb{R}^{n}\right)$ and find bases and degrees of freedom for them, and also characterize them through affine invariance. We then assemble these spaces into finite element differential forms, which are the main objects of interest, and derive the finite element de Rham subcomplexes and cochain projections which are the key tools of finite element exterior calculus. With these we easily present the basic finite element discrete Hodge theory.

In the second part of the paper, we apply these tools to concrete problems: discretization of the Hodge Laplacian, eigenvalue problems, Maxwell's equations, and preconditioning. The final application, and the most substantial, is to mixed discretizations of elasticity. Here we use some additional tools: vector-valued differential forms and the BGG resolution.

\section{PART ONE}

\section{Exterior calculus, finite elements, and homology}

\section{Exterior algebra and exterior calculus}

In this section we recall the basic objects and results of exterior algebra and exterior calculus. This material can be found, in varying degrees of generality and in varying notation, in many sources, including Arnold (1978), Bott and Tu (1982), Federer (1969), Jänich (2001), Lang (1995), and Taylor (1996). 


\subsection{Exterior algebra}

Alternating algebraic forms on a vector space

Let $V$ be a real vector space of dimension $n$. For each positive integer $k$, we denote by $\mathrm{Alt}^{k} V$ the space of alternating $k$-linear maps $V \times \cdots \times V \rightarrow \mathbb{R}$. We refer to such maps as alternating algebraic $k$-forms on $V$ or simply algebraic $k$-forms. Thus, an algebraic $k$-form on $V$ assigns to a $k$-tuple $\left(v_{1}, \ldots, v_{k}\right)$ of elements of $V$ a real number $\omega\left(v_{1}, \ldots, v_{k}\right)$, with the mapping linear in each argument, and reversing sign when any two arguments are interchanged. It is natural to set $\mathrm{Alt}^{0} V=\mathbb{R}$. Note that $\operatorname{Alt}^{1} V$ is just the dual space $V^{*}$ of $V$, the space of covectors.

Exterior product

Given $\omega \in \mathrm{Alt}^{j} V$ and $\eta \in \mathrm{Alt}^{k} V$, we define their exterior product or wedge product $\omega \wedge \eta \in \mathrm{Alt}^{j+k} V$ by

$$
\begin{aligned}
(\omega & \wedge \eta)\left(v_{1}, \ldots, v_{j+k}\right) \\
& =\sum_{\sigma}(\operatorname{sign} \sigma) \omega\left(v_{\sigma(1)}, \ldots, v_{\sigma(j)}\right) \eta\left(v_{\sigma(j+1)}, \ldots, v_{\sigma(j+k)}\right), \quad v_{i} \in V
\end{aligned}
$$

where the sum is over all permutations $\sigma$ of $\{1, \ldots, j+k\}$, for which $\sigma(1)<$ $\sigma(2)<\cdots \sigma(j)$ and $\sigma(j+1)<\sigma(j+2)<\cdots \sigma(j+k)$. The exterior product is bilinear and associative, and satisfies the anti-commutativity condition

$$
\eta \wedge \omega=(-1)^{j k} \omega \wedge \eta, \quad \omega \in \operatorname{Alt}^{j} V, \eta \in \operatorname{Alt}^{k} V .
$$

This can be summarized by the statement that the direct sum Alt $V:=$ $\bigoplus_{k} \mathrm{Alt}^{k} V$ is a anti-commutative graded algebra called the Grassmann algebra or exterior algebra of $V^{*}$. (In the context of graded algebras, anticommutativity is often referred to simply as commutativity.)

\section{Pullback}

A linear transformation of vector spaces $L: V \rightarrow W$ induces a linear transformation $L^{*}:$ Alt $W \rightarrow$ Alt $V$ called the pullback, given by

$$
L^{*} \omega\left(v_{1}, \ldots, v_{k}\right)=\omega\left(L v_{1}, \ldots, L v_{k}\right), \quad \omega \in \operatorname{Alt}^{k} W, \quad v_{1}, \ldots, v_{k} \in V .
$$

The pullback acts contravariantly: if $U \stackrel{K}{\rightarrow} V \stackrel{L}{\rightarrow} W$, then Alt $W \stackrel{L^{*}}{\longrightarrow}$ Alt $V \stackrel{K^{*}}{\longrightarrow}$ Alt $U$, and $K^{*} \circ L^{*}=(L \circ K)^{*}$. The pullback also respects the algebra structure in the sense that $L^{*}(\omega \wedge \eta)=L^{*} \omega \wedge L^{*} \eta$.

A particular case is when $L$ is the inclusion $i_{V}$ of a subspace $V$ into $W$. Then the pullback defines a surjection $i_{V}^{*}$ of Alt $W$ onto Alt $V$. If in addition $W$ has an inner product, so that the orthogonal projection $\pi_{V}: W \rightarrow V$ is defined, then its pullback defines an injection of Alt $V$ into Alt $W$ and the pullback of the composition $W \stackrel{\pi_{V}}{\longrightarrow} V \stackrel{i}{\rightarrow} W$ associates to each $\omega \in \mathrm{Alt}^{k} W$ 
its tangential part with respect to $V$, given by

$$
\left(\pi_{V}^{*} i_{V}^{*} \omega\right)\left(v_{1}, \ldots, v_{k}\right)=\omega\left(\pi_{V} v_{1}, \ldots, \pi_{V} v_{k}\right) .
$$

Thus the tangential part of $\omega$ vanishes if and only the image of $\omega$ in Alt $^{k} V$ vanishes. We may also speak of the normal part of $\omega$ with respect to $V$, defined to be $\omega-\pi_{V}^{*} i_{V}^{*} \omega$. (For $k>1$, this is not generally the same as the tangential part with respect to the orthogonal complement of $V$.)

\section{Bases}

Let $v_{1}, \ldots, v_{n}$ be some basis of $V$. Then an algebraic $k$-form $\omega$ is uniquely determined by its values $\omega\left(v_{\sigma(1)}, \ldots, v_{\sigma(k)}\right)$ for each increasing map $\sigma$ : $\{1, \ldots, k\} \rightarrow\{1, \ldots, n\}$, and these values may be assigned arbitrarily. Thus

$$
\operatorname{dim} \operatorname{Alt}^{k} V=\left(\begin{array}{l}
n \\
k
\end{array}\right)
$$

and, in particular, $\mathrm{Alt}^{k} V=0$ for $k>n$.

Associated to the given basis is the dual basis $\mu_{1}, \ldots, \mu_{n}$ for $V^{*}$, defined by $\mu_{i}\left(v_{j}\right)=\delta_{i j}$. For $\sigma, \rho:\{1, \ldots, k\} \rightarrow\{1, \ldots, n\}$ increasing, we have

$$
\mu_{\sigma(1)} \wedge \cdots \wedge \mu_{\sigma(k)}\left(v_{\rho(1)}, \ldots, v_{\rho(k)}\right)= \begin{cases}1, & \text { if } \sigma=\rho, \\ 0, & \text { otherwise }\end{cases}
$$

so the $\left(\begin{array}{l}n \\ k\end{array}\right)$ algebraic $k$-forms $\mu_{\sigma(1)} \wedge \cdots \wedge \mu_{\sigma(k)}$ form a basis for Alt $^{k} V$ naturally associated to the given basis of $V$.

\section{Interior product}

To an algebraic $k$-form $\omega$ and a vector $v \in V$, we may associate an algebraic $(k-1)$-form $\omega\lrcorner v$ called the interior product, or the contraction of $\omega$ by $v$, defined by

$$
\omega\lrcorner v\left(v_{1}, \ldots, v_{k-1}\right)=\omega\left(v, v_{1}, \ldots, v_{k-1}\right) .
$$

(We take $\omega\lrcorner v$ to be 0 if $\omega$ is an algebraic 0 -form.) Since the forms are alternating, repeated contraction vanishes:

$$
(\omega\lrcorner v)\lrcorner v=0, \quad \omega \in \mathrm{Alt}^{k} V .
$$

Moreover, it is easy to check that

$$
\left.(\omega \wedge \eta)\lrcorner v=(\omega\lrcorner v) \wedge \eta+(-1)^{k} \omega \wedge(\eta\lrcorner v\right), \quad \omega \in \mathrm{Alt}^{k} V, \eta \in \mathrm{Alt}^{l} V .
$$

Inner product, orientation, and volume form

If the vector space $V$ is endowed with an inner product, then $\mathrm{Alt}^{k} V$ is naturally endowed with an inner product by the formula

$$
\langle\omega, \eta\rangle=\sum_{\sigma} \omega\left(v_{\sigma(1)}, \ldots, v_{\sigma(k)}\right) \eta\left(v_{\sigma(1)}, \ldots, v_{\sigma(k)}\right), \quad \omega, \eta \in \mathrm{Alt}^{k} V
$$


where the sum is over increasing sequences $\sigma:\{1, \ldots, k\} \rightarrow\{1, \ldots, n\}$ and $v_{1}, \ldots, v_{n}$ is any orthonormal basis (the right-hand side being independent of the choice of orthonormal basis). The space $\operatorname{Alt}^{n} V, n=\operatorname{dim} V$ has dimension 1, and we uniquely determine an element in it by giving its value on a single ordered basis. Its value on any list of $n$ vectors is then the same value multiplied by the determinant of the matrix expressing the vectors in terms of the specified basis. In particular, we determine an algebraic $n$-form, unique up to sign, by requiring it to take the value 1 on some orthonormal basis. It will then take the values \pm 1 on all orthonormal bases. We fix the sign by orienting the vector space, i.e., by designating one ordered basis as positively oriented (and then all bases will be positively or negatively oriented according to the sign of the determinant of the change-of-basis matrix). The resulting uniquely determined algebraic $n$-form is called the volume form on the oriented vector space $V$.

\section{Bivectors}

When we treat the equations of elasticity, we will make use of the space $V \wedge V$ of bivectors, which we now define. (There is an entire algebra of multivectors, in analogy to the exterior algebra of multi-covectors, but we shall not have need of this.)

For $v$ and $w$ elements of a vector space $V$, define $v \wedge w=v \otimes w-w \otimes v \in$ $V \otimes V$, and let $V \wedge V$ denote the subspace of $V \otimes V$ spanned by elements of the form $v \wedge w$. If $v_{1}, \ldots, v_{n}$ denotes a basis of $V$, then the vectors $v_{i} \wedge v_{j}$, $i<j$, form a basis for $V \wedge V$, and so $\operatorname{dim} V \wedge V=\left(\begin{array}{l}n \\ 2\end{array}\right)$. The mapping $v \otimes w \mapsto(v \wedge w) / 2$ defines a linear operator skw $: V \otimes V \rightarrow V \wedge V$.

The space $V \otimes V$ is identified with $\mathcal{L}\left(V^{*}, V\right)$, the space of linear operators from $V^{*}$ to $V$, by $(v \otimes w)(f)=f(w) v$. If we assume that $V$ has an inner product, then $V^{*}$ is identified with $V$, and so $V \otimes V$ with $\mathcal{L}(V, V)$. The subspace $V \wedge V$ is then the subspace of linear operators which are skewsymmetric with respect to the inner product and skw is the map which takes a linear operator to its skew-symmetric part.

\section{The Hodge star operation}

Let $V$ be an oriented inner product space with volume form vol. Given $\omega \in \mathrm{Alt}^{k} V$, we obtain a linear map $L_{\omega}: \mathrm{Alt}^{n-k} V \rightarrow \mathbb{R}$, by composing the map $\mu \mapsto \omega \wedge \mu$ with the canonical isomorphism of $\Lambda^{n} V$ onto $\mathbb{R}$ (given by $c$ vol $\mapsto c)$. By the Riesz representation theorem, there exists an element $\star \omega \in \mathrm{Alt}^{n-k} V$ such that $L_{\omega}(\mu)=\langle\star \omega, \mu\rangle$. In other words,

$$
\omega \wedge \mu=\langle\star \omega, \mu\rangle \text { vol, } \omega \in \mathrm{Alt}^{k} V, \mu \in \mathrm{Alt}^{n-k} V .
$$

The linear map $\omega \mapsto \star \omega$ taking $\mathrm{Alt}^{k} V$ into $\mathrm{Alt}^{n-k} V$ is the Hodge star operator; there is one for each $k$ with $0 \leq k \leq n$. From the definition we 
find that

$$
\omega\left(e_{\sigma(1)}, \ldots, e_{\sigma(k)}\right)=(\operatorname{sign} \sigma) \star \omega\left(e_{\sigma(k+1)}, \ldots, e_{\sigma(n)}\right),
$$

for any positively oriented orthonormal basis $e_{1}, \ldots, e_{k}$ and any permutation $\sigma$.

Applying this, we see that

$$
\star(\star \omega)=(-1)^{k(n-k)} \omega, \quad \omega \in \mathrm{Alt}^{k} V,
$$

and, consequently, that the Hodge star operator is an isometry.

Let $W$ be a subspace of $V$, and let $\omega=\omega_{\|}+\omega_{\perp}$ denote the decomposition of an algebraic form into its tangential and normal parts with respect to $W$. By taking an orthonormal basis of $W$ and extending it to an orthonormal basis of $V$, we find that

$$
(\star \omega)_{\|}=\star\left(\omega_{\perp}\right), \quad(\star \omega)_{\perp}=\star\left(\omega_{\|}\right) .
$$

In particular, the image of $\star \omega$ in $\mathrm{Alt}^{k} W$ (under the pullback of the inclusion) vanishes if and only if the normal component of $\omega$ vanishes.

There is also a Hodge star operation for multivectors. In two dimensions it takes bivectors to scalars (by $e_{1} \wedge e_{2} \mapsto 1$ ) and in three dimensions it takes bivectors to vectors (via $e_{1} \wedge e_{2} \mapsto e_{3}$, etc.)

The case $V=\mathbb{R}^{n}$

Finally, we consider the case $V=\mathbb{R}^{n}$, and note that we have a natural identification of $\mathrm{Alt}^{0} \mathbb{R}^{n}$ and $\mathrm{Alt}^{n} \mathbb{R}^{n}$ with $\mathbb{R}$. In fact, $\mathrm{Alt}^{0} \mathbb{R}^{n}$ is defined to equal $\mathbb{R}$, while the general element $\omega \in \mathrm{Alt}^{n} \mathbb{R}^{n}$ can be written

$$
\omega\left(v_{1}, \ldots, v_{n}\right)=c \operatorname{det}\left[v_{1}|\cdots| v_{n}\right],
$$

for some $c \in \mathbb{R}$, so $\omega \leftrightarrow c$ is the desired identification. For $n>1$, we also have natural identifications of $\mathrm{Alt}^{1} \mathbb{R}^{n}$ and $\mathrm{Alt}^{n-1} \mathbb{R}^{n}$ with $\mathbb{R}^{n}$. The identification of $\mathrm{Alt}^{1} \mathbb{R}^{n}$ with $\mathbb{R}^{n}$ is the usual Riesz identification of $V^{*}$ with $V$ based on an inner product on $V$ (for $\mathbb{R}^{n}$ we use the Euclidean inner product, of course). The identification of $\mathrm{Alt}^{n-1} \mathbb{R}^{n}$ with $\mathbb{R}^{n}$ identifies a vector $v \in \mathbb{R}^{n}$ with the algebraic $(n-1)$-form

$$
\left(v_{1}, \ldots, v_{n-1}\right) \mapsto \operatorname{det}\left[v\left|v_{1}\right| \cdots \mid v_{n-1}\right] .
$$

The canonical basis $e_{1}, \ldots, e_{n}$ of $\mathbb{R}^{n}$ gives rise to a dual basis of $\left(\mathbb{R}^{n}\right)^{*}$ which will often be denoted $\mathrm{d} x_{1}, \ldots, \mathrm{d} x_{n}$ (the reason for this notation will be made clear in Section 2.3). Thus $\mathrm{d} x_{i}\left(e_{j}\right)=\delta_{i j}$ and $\mathrm{d} x_{i}(v)=e_{i} \cdot v=v_{i}$.

For $n=3$, each of the spaces $\mathrm{Alt}^{k} \mathbb{R}^{3}$ may be identified either with $\mathbb{R}$ $(k=0,3)$ or with $\mathbb{R}^{3}(k=1,2)$. Thus all the operations on exterior forms correspond to operations on scalars and/or vectors. The correspondences are listed in Table 2.1.

A similar set of correspondences exist for $n=2$, although in this case, for 
Table 2.1. Correspondence between alternating algebraic forms on $\mathbb{R}^{3}$ and scalars/vectors.

\begin{tabular}{|c|c|}
\hline \multicolumn{2}{|c|}{ correspondence } \\
\hline $\operatorname{Alt}^{0} \mathbb{R}^{3}=\mathbb{R}$ & $c \leftrightarrow c$ \\
\hline $\operatorname{Alt}^{1} \mathbb{R}^{3} \stackrel{\cong}{\longrightarrow} \mathbb{R}^{3}$ & $u_{1} \mathrm{~d} x_{1}+u_{2} \mathrm{~d} x_{2}+u_{3} \mathrm{~d} x_{3} \leftrightarrow u$ \\
\hline $\operatorname{Alt}^{2} \mathbb{R}^{3} \stackrel{\cong}{\longrightarrow} \mathbb{R}^{3}$ & $\begin{aligned} u_{3} \mathrm{~d} x_{1} \wedge \mathrm{d} x_{2}-u_{2} \mathrm{~d} x_{1} \wedge \mathrm{d} x_{3} \\
+u_{1} \mathrm{~d} x_{2} \wedge \mathrm{d} x_{3} \leftrightarrow u\end{aligned}$ \\
\hline $\mathrm{Alt}^{3} \mathbb{R}^{3} \stackrel{\cong}{\longrightarrow} \mathbb{R}$ & $c \leftrightarrow c \mathrm{~d} x_{1} \wedge \mathrm{d} x_{2} \wedge \mathrm{d} x_{3}$ \\
\hline
\end{tabular}

exterior product

\begin{tabular}{l|l}
\hline$\wedge: \operatorname{Alt}^{1} \mathbb{R}^{3} \times \mathrm{Alt}^{1} \mathbb{R}^{3} \rightarrow \mathrm{Alt}^{2} \mathbb{R}^{3}$ & $\times: \mathbb{R}^{3} \times \mathbb{R}^{3} \rightarrow \mathbb{R}^{3}$ \\
$\wedge: \mathrm{Alt}^{1} \mathbb{R}^{3} \times \mathrm{Alt}^{2} \mathbb{R}^{3} \rightarrow \mathrm{Alt}^{3} \mathbb{R}^{3}$ & $\cdot: \mathbb{R}^{3} \times \mathbb{R}^{3} \rightarrow \mathbb{R}$ \\
\hline \multicolumn{1}{c}{ pullback by a linear map $L: \mathbb{R}^{3} \rightarrow \mathbb{R}^{3}$} \\
\hline \multicolumn{2}{c}{ id $: \mathbb{R} \rightarrow \mathbb{R}$} \\
$L^{*}: \mathrm{Alt}^{0} \mathbb{R}^{3} \rightarrow \mathrm{Alt}^{0} \mathbb{R}^{3}$ & $L^{T}: \mathbb{R}^{3} \rightarrow \mathbb{R}^{3}$ \\
$L^{*}: \mathrm{Alt}^{1} \mathbb{R}^{3} \rightarrow \mathrm{Alt}^{1} \mathbb{R}^{3}$ & $(\operatorname{det} L) L^{-1}: \mathbb{R}^{3} \rightarrow \mathbb{R}^{3}$ \\
$L^{*}: \mathrm{Alt}^{2} \mathbb{R}^{3} \rightarrow \mathrm{Alt}^{2} \mathbb{R}^{3}$ & $(\operatorname{det} L): \mathbb{R} \rightarrow \mathbb{R} \quad(c \mapsto c \operatorname{det} L)$ \\
$L^{*}: \mathrm{Alt}^{3} \mathbb{R}^{3} \rightarrow \mathrm{Alt}^{3} \mathbb{R}^{3}$ & \\
\hline
\end{tabular}

interior product with a vector $v \in \mathbb{R}^{3}$

\begin{tabular}{l|l}
\lrcorner $v: \mathrm{Alt}^{1} \mathbb{R}^{3} \rightarrow \mathrm{Alt}^{0} \mathbb{R}^{3}$ & $v \cdot: \mathbb{R}^{3} \rightarrow \mathbb{R}$ \\
\lrcorner $v: \mathrm{Alt}^{2} \mathbb{R}^{3} \rightarrow \mathrm{Alt}^{1} \mathbb{R}^{3}$ & $v \times: \mathbb{R}^{3} \rightarrow \mathbb{R}^{3}$ \\
\lrcorner $v: \mathrm{Alt}^{3} \mathbb{R}^{3} \rightarrow \mathrm{Alt}^{2} \mathbb{R}^{3}$ & $v: \mathbb{R} \rightarrow \mathbb{R}^{3} \quad(c \mapsto c v)$
\end{tabular}

inner product and volume form

inner product on $\mathrm{Alt}^{k} \mathbb{R}^{3}$ induced $\quad$ dot product on $\mathbb{R}$ and $\mathbb{R}^{3}$ by dot product on $\mathbb{R}^{3}$

\begin{tabular}{l|l}
$\mathrm{vol}=\mathrm{d} x_{1} \wedge \mathrm{d} x_{2} \wedge \mathrm{d} x_{3}$ & $\left(v_{1}, v_{2}, v_{3}\right) \mapsto \operatorname{det}\left[v_{1}\left|v_{2}\right| v_{3}\right]$
\end{tabular}

Hodge star
$\star: \mathrm{Alt}^{0} \mathbb{R}^{3} \rightarrow \mathrm{Alt}^{3} \mathbb{R}^{3}$
id $: \mathbb{R} \rightarrow \mathbb{R}$
$\star: \mathrm{Alt}^{1} \mathbb{R}^{3} \rightarrow \mathrm{Alt}^{2} \mathbb{R}^{3}$
id $: \mathbb{R}^{3} \rightarrow \mathbb{R}^{3}$ 
operations involving $\mathrm{Alt}^{1} \mathbb{R}^{2}$ there are two possibilities since there are two different identifications of $\mathrm{Alt}^{1} \mathbb{R}^{2}$ with $\mathbb{R}^{2}$ (as 1 -forms or as $(n-1)$-forms).

\subsection{Exterior calculus on manifolds}

\section{Manifolds}

The natural setting for exterior calculus is a (sufficiently) smooth manifold $\Omega$ of finite dimension $n$, with or without boundary. At each point $x \in \Omega$, the tangent space $T_{x} \Omega$ is a vector space of dimension $n$. The tangent bundle consists of all pairs $(x, v)$ with $x \in \Omega, v \in T_{x} \Omega$. It is a smooth manifold of dimension $2 n$. The sections of this bundle, that is the maps $x \in \Omega \mapsto v(x) \in T_{x} \Omega$, are the vector fields on $\Omega$.

If $\phi: \Omega \rightarrow \Omega^{\prime}$ is a smooth map of manifolds, and $x \in \Omega$, then the tangent map $D \phi_{x}$ is a linear map from $T_{x} \Omega$ to $T_{\phi(x)} \Omega^{\prime}$. In case $\Omega^{\prime}=\mathbb{R}$, so $\phi$ is a smooth scalar-valued function on $\Omega$, we write $\partial_{v} \phi(x)$ for $D \phi_{x}(v), x \in \Omega$, $v \in T_{x} \Omega$, the directional derivative of $\phi$ at $x$ in the direction given by $v$. In case $\Omega \subset \mathbb{R}$, so $\phi$ is a curve in $\Omega^{\prime}$, we write $\mathrm{d} \phi(t) / \mathrm{d} t=D \phi_{t}(1)$.

\section{Differential forms}

Applying the exterior algebra construction to the tangent spaces, we obtain the exterior forms bundle, a smooth manifold whose elements are pairs $(x, \mu)$ with $x \in \Omega, \mu \in \mathrm{Alt}^{k} T_{x} \Omega$. A differential $k$-form is a section of this bundle, i.e., a map $\omega$ which associates to each $x \in \Omega$ an element $\omega_{x} \in \operatorname{Alt}^{k} T_{x} \Omega$. Thus, if $\omega$ is a differential $k$-form on $\Omega, x \in \Omega$, and $v_{1}, \ldots, v_{k} \in T_{x} \Omega$, then $\omega_{x}\left(v_{1}, \ldots, v_{k}\right) \in \mathbb{R}$. If the map

$$
x \in \Omega \mapsto \omega_{x}\left(v_{1}(x), \ldots, v_{k}(x)\right) \in \mathbb{R},
$$

is smooth (infinitely differentiable) whenever the $v_{i}$ are smooth vector fields, then we say that $\omega$ is a smooth differential $k$-form. We denote by $\Lambda^{k}(\Omega)$ the space of all smooth differential $k$-forms on $\Omega$. Note that $\Lambda^{0}(\Omega)=C^{\infty}(\Omega)$ and $\Lambda^{1}(\Omega)$ is the space of smooth covector fields.

\section{Exterior product}

For each $k, \Lambda^{k}(\Omega)$ is not only an (infinite-dimensional) vector space, but also a module with respect to the ring $C^{\infty}(\Omega)$ of smooth functions on $\Omega$ : if $\omega \in \Lambda^{k}(\Omega)$ and $f \in C^{\infty}(\Omega)$, the product $f \omega$ belongs to $\Lambda^{k}(\Omega)$. The exterior product of algebraic forms may be applied pointwise to define the exterior product of differential forms:

$$
(\omega \wedge \eta)_{x}=\omega_{x} \wedge \eta_{x} .
$$

In this way we obtain the anti-commutative graded algebra

$$
\Lambda(\Omega)=\bigoplus_{k} \Lambda^{k}(\Omega)
$$




\section{$C^{m}$ spaces of differentiable differential forms}

We may also consider spaces of differential forms with less smoothness. If the map (2.3) merely belongs to $C^{m}(\Omega)$ for some $m \geq 0$ whenever the $v_{i}$ are smooth vector fields, then we say that $\omega$ is a $C^{m}$ differential $k$-form, the space of all such we denote by $C^{m} \Lambda^{k}(\Omega)$.

\section{Integration of differential forms}

Differential forms can be integrated and differentiated without recourse to any additional structure, such as a measure or a metric, on the manifold $\Omega$. If $f$ is an oriented, piecewise smooth $k$-dimensional submanifold of the manifold $\Omega$, and $\omega$ is a continuous $k$-form, then the integral $\int_{f} \omega$ is welldefined. Thus, for example, 0 -forms can be evaluated at points, 1-forms can be integrated over directed curves, and 2 -forms can be integrated over oriented surfaces.

\section{Exterior differentiation}

The exterior derivative $\mathrm{d}: \Lambda(\Omega) \rightarrow \Lambda(\Omega)$ is a graded linear operator of degree +1 , i.e., $\mathrm{d}$ maps $\Lambda^{k}(\Omega)$ into $\Lambda^{k+1}(\Omega)$ for each $k \geq 0$. We give a formula for $\Omega$ a domain in $\mathbb{R}^{n}$. In this case, we may identify each tangent space $T_{x} \Omega$ with $\mathbb{R}^{n}$, and hence for given $\omega \in \Lambda^{k}(\Omega)$ and vectors $v_{1}, \ldots, v_{k}$, obtain a smooth mapping $\Omega \rightarrow \mathbb{R}$ given by

$$
x \mapsto \omega_{x}\left(v_{1}, \ldots, v_{k}\right) .
$$

We then define

$$
\begin{aligned}
\mathrm{d} \omega_{x}\left(v_{1}, \ldots, v_{k+1}\right)=\sum_{j=1}^{k+1}(-1)^{j+1} \partial_{v_{j}} \omega_{x}\left(v_{1}, \ldots, \hat{v}_{j}, \ldots, v_{k+1}\right), & \\
& \omega \in \Lambda^{k}, v_{1}, \ldots, v_{k+1} \in V,
\end{aligned}
$$

where the hat is used to indicate a suppressed argument. For $\Omega$ a general manifold, a similar but more involved expression can be used to define exterior differentiation. In this case the vectors $v_{i}$ must be replaced by smoothly varying vector fields, and additional terms arise due to the noncommutativity of the vector fields. See Lang (1995, Chapter V, Section 3).

We recall two key properties of exterior differentiation. It is a differential: $\mathrm{d} \circ \mathrm{d}=0$; and it satisfies a Leibniz rule with respect to the wedge product:

$$
\mathrm{d}(\omega \wedge \eta)=\mathrm{d} \omega \wedge \eta+(-1)^{j} \omega \wedge \mathrm{d} \eta, \quad \omega \in \Lambda^{j}(\Omega), \quad \eta \in \Lambda^{k}(\Omega) .
$$

\section{Pullback}

A smooth map $\phi: \Omega \rightarrow \Omega^{\prime}$ between manifolds, provides a pullback of differential forms from $\Omega^{\prime}$ to $\Omega$. Namely, if $\omega$ is a differential $k$-form on $\Omega^{\prime}$, 
we define the pullback $\phi^{*} \omega \in \Lambda^{k}(\Omega)$ by

$$
\left(\phi^{*} \omega\right)_{x}=\left(D \phi_{x}\right)^{*} \omega_{\phi(x)}
$$

i.e.,

$$
\left(\phi^{*} \omega\right)_{x}\left(v_{1}, \ldots, v_{k}\right)=\omega_{\phi(x)}\left(D \phi_{x}\left(v_{1}\right), \ldots, D \phi_{x}\left(v_{k}\right)\right) .
$$

The pullback respects exterior products and differentiation:

$$
\phi^{*}(\omega \wedge \eta)=\phi^{*} \omega \wedge \phi^{*} \eta, \quad \phi^{*}(\mathrm{~d} \omega)=\mathrm{d}\left(\phi^{*} \omega\right), \quad \omega, \eta \in \Lambda\left(\Omega^{\prime}\right) .
$$

If $\phi$ is an orientation-preserving diffeomorphism, then we also have

$$
\int_{\Omega} \phi^{*} \omega=\int_{\Omega^{\prime}} \omega, \quad \omega \in \Lambda^{n}\left(\Omega^{\prime}\right) .
$$

If $\Omega^{\prime}$ is a submanifold of $\Omega$, then the pullback of the inclusion $\Omega^{\prime} \hookrightarrow \Omega$ is the trace map $\operatorname{Tr}_{\Omega, \Omega^{\prime}}: \Lambda(\Omega) \rightarrow \Lambda\left(\Omega^{\prime}\right)$. If the domain $\Omega$ is clear from context, we may write $\operatorname{Tr}_{\Omega^{\prime}}$ instead of $\operatorname{Tr}_{\Omega, \Omega^{\prime}}$, and we usually abbreviate $\operatorname{Tr}_{\Omega, \partial \Omega}$ as simply Tr. Note that, if $\Omega^{\prime}$ is a submanifold of positive codimension and $k>0$, then the vanishing of $\operatorname{Tr}_{\Omega, \Omega^{\prime}} \omega$ on $\Omega^{\prime}$ for $\omega \in \Lambda^{k}(\Omega)$ does not imply that $\omega_{x} \in \operatorname{Alt}^{k} T_{x} \Omega$ vanishes for $x \in \Omega^{\prime}$, only that it vanishes when applied to $k$-tuples of vectors tangent to $\Omega^{\prime}$, or, in other words, that the tangential part of $\omega_{x}$ with respect to $T_{x} \Omega^{\prime}$ vanishes.

\section{Stokes' theorem and integration by parts}

Integration of differential forms and exterior differentiation are related via Stokes' theorem. If $\Omega$ is an oriented $n$-manifold with boundary $\partial \Omega$ (endowed with the induced orientation), then

$$
\int_{\Omega} \mathrm{d} \omega=\int_{\partial \Omega} \operatorname{Tr} \omega, \quad \omega \in \Lambda^{k-1}(\Omega) .
$$

Combining with the Leibniz rule, we get the integration by parts formula

$$
\int_{\Omega} \mathrm{d} \omega \wedge \eta=(-1)^{k-1} \int_{\Omega} \omega \wedge \mathrm{d} \eta+\int_{\partial \Omega} \operatorname{Tr} \omega \wedge \operatorname{Tr} \eta
$$

if $\omega \in \Lambda^{k}(\Omega), \eta \in \Lambda^{n-k-1}(\Omega)$.

\section{Interior product}

Clearly we may form the interior product of a differential $k$-form $\omega$ with a vector field $v$, to obtain a $(k-1)$-form: $\left.(\omega\lrcorner v)_{x}=\omega_{x}\right\lrcorner v_{x}$.

\section{Inner product}

Suppose $\Omega$ is an oriented Riemannian manifold, so that each tangent space is endowed with an inner product, and so also are the spaces $\operatorname{Alt}^{k} T_{x} \Omega$. Moreover, there is a unique volume form, vol in $\Lambda^{n}(\Omega)$ such that at each 
$x \in \Omega, \operatorname{vol}_{x}$ is the volume form associated with the oriented inner product space $T_{x} \Omega$. Therefore we can define the integral of any function $f \in \Lambda^{0}(\Omega)$ simply as $\int_{\Omega} f$ vol. In particular, we can define the $L^{2}$ inner product of any two differential $k$-forms on $\Omega$ as the integral of their pointwise inner product:

$$
\langle\omega, \eta\rangle_{L^{2} \Lambda^{k}}=\int_{\Omega}\left\langle\omega_{x}, \eta_{x}\right\rangle \mathrm{vol}=\int \omega \wedge \star \eta .
$$

The completion of $\Lambda^{k}(\Omega)$ in the corresponding norm defines the Hilbert space $L^{2} \Lambda^{k}(\Omega)$.

\section{Sobolev spaces of differential forms}

On an oriented Riemannian manifold, we may also define the Sobolev spaces $H^{s}(\Omega)$ and $W^{s, p}(\Omega)$ of functions with $s \geq 0$ derivatives in $L^{2}(\Omega)$ and $L^{p}(\Omega)$, respectively. We may then define the spaces $H^{s} \Lambda^{k}(\Omega)$ consisting of differential forms for which the quantities in (2.3) belong to $H^{s}(\Omega)$. These Sobolev spaces are Hilbert spaces.

For a differential form to belong to $H^{s} \Lambda^{k}(\Omega)$, all its partial derivatives of order at most $s$ (in some coordinate system) must be square integrable. A different notion is obtained by only considering the exterior derivatives. We define another Hilbert space:

$$
H \Lambda^{k}(\Omega)=\left\{\omega \in L^{2} \Lambda^{k}(\Omega) \mid \mathrm{d} \omega \in L^{2} \Lambda^{k+1}(\Omega)\right\} .
$$

The norm is defined by

$$
\|\omega\|_{H \Lambda^{k}}^{2}=\|\omega\|_{H \Lambda}^{2}:=\|\omega\|_{L^{2} \Lambda^{k}}^{2}+\|\mathrm{d} \omega\|_{L^{2} \Lambda^{k+1}}^{2} .
$$

The space $H \Lambda^{0}(\Omega)$ coincides with $H^{1} \Lambda^{0}(\Omega)$ (or simply $H^{1}(\Omega)$ ), while the space $H \Lambda^{n}(\Omega)$ coincides with $L^{2} \Lambda^{n}(\Omega)$. For $0<k<n, H \Lambda^{k}(\Omega)$ is contained strictly between $H^{1} \Lambda^{k}(\Omega)$ and $L^{2} \Lambda^{k}(\Omega)$.

The de Rham complex

The de Rham complex is the sequence of spaces and mappings

$$
0 \rightarrow \Lambda^{0}(\Omega) \stackrel{\mathrm{d}}{\rightarrow} \Lambda^{1}(\Omega) \stackrel{\mathrm{d}}{\rightarrow} \cdots \stackrel{\mathrm{d}}{\rightarrow} \Lambda^{n}(\Omega) \rightarrow 0 .
$$

Since $\mathrm{d} \circ \mathrm{d}=0$, we have

$$
\left.\mathcal{R}\left(\mathrm{d}: \Lambda^{k-1}(\Omega) \rightarrow \Lambda^{k}(\Omega)\right) \subset \mathcal{N}\left(\mathrm{d}: \Lambda^{k}(\Omega) \rightarrow \Lambda^{k+1}(\Omega)\right)\right),
$$

for $k=0,1, \ldots, n$, which is to say that this sequence is a cochain complex. The de Rham cohomology spaces are the quotient spaces. They are finitedimensional vector spaces whose dimensions are the Betti numbers of the manifold $\Omega$. For a bounded connected region in $\mathbb{R}^{3}$, the zeroth Betti number is 1, the first Betti number is the genus (number of handles), the second 
Betti number is one less than the number of connected components of the boundary (number of holes), and the third Betti number is 0 .

For an oriented Riemannian manifold, we obtain the same cohomology spaces from the $L^{2}$ de Rham complex ${ }^{1}$ :

$$
0 \rightarrow H \Lambda^{0}(\Omega) \stackrel{\mathrm{d}}{\rightarrow} H \Lambda^{1}(\Omega) \stackrel{\mathrm{d}}{\rightarrow} \cdots \stackrel{\mathrm{d}}{\rightarrow} H \Lambda^{n}(\Omega) \rightarrow 0 .
$$

The $k$ th cohomology space is isomorphic to the space of $\mathfrak{H}^{k}(\Omega)$ of harmonic $k$-forms on $\Omega$ :

$$
\mathfrak{H}^{k}(\Omega)=\left\{\omega \in H \Lambda^{k}(\Omega) \mid \mathrm{d} \omega=0,\langle\omega, \mathrm{d} \eta\rangle_{L^{2} \Lambda^{k}}=0 \forall \eta \in H \Lambda^{k-1}\right\} .
$$

The isomorphism simply associates to a harmonic form $\omega$ the cohomology class it represents.

In the case of a contractible domain $\Omega$, all the cohomology spaces vanish, except the one of lowest order, which is the constants. In other words, the extended de Rham complex

$$
0 \rightarrow \mathbb{R} \stackrel{\subset}{\rightarrow} \Lambda^{0}(\Omega) \stackrel{\mathrm{d}}{\rightarrow} \Lambda^{1}(\Omega) \stackrel{\mathrm{d}}{\rightarrow} \cdots \stackrel{\mathrm{d}}{\rightarrow} \Lambda^{n}(\Omega) \rightarrow 0
$$

is exact.

The coderivative operator

The coderivative operator $\delta: \Lambda^{k}(\Omega) \rightarrow \Lambda^{k-1}(\Omega)$ is defined

$$
\star \delta \omega=(-1)^{k} \mathrm{~d} \star \omega, \quad \omega \in \Lambda^{k}(\Omega) .
$$

It follows directly from $(2.4),(2.5)$, and (2.7), that

$$
\langle\mathrm{d} \omega, \eta\rangle=\langle\omega, \delta \eta\rangle+\int_{\partial \Omega} \operatorname{Tr} \omega \wedge \operatorname{Tr} \star \eta, \quad \omega \in \Lambda^{k}(\Omega), \eta \in \Lambda^{k+1}(\Omega) .
$$

Thus $\delta: \Lambda^{k+1}(\Omega) \rightarrow \Lambda^{k}(\Omega)$ is the formal adjoint of $\mathrm{d}: \Lambda^{k}(\Omega) \rightarrow \Lambda^{k+1}(\Omega)$ with respect to the $L^{2}$-inner product: we have

$$
\langle\mathrm{d} \omega, \eta\rangle=\langle\omega, \delta \eta\rangle
$$

if $\omega$ or $\eta$ vanish near the boundary.

In analogy with $H \Lambda^{k}(\Omega)$, we define the space

$$
H^{*} \Lambda^{k}(\Omega)=\left\{\omega \in L^{2} \Lambda^{k}(\Omega) \mid \delta \omega \in L^{2} \Lambda^{k-1}(\Omega)\right\} .
$$

Clearly $H^{*} \Lambda^{k}(\Omega)=\star H \Lambda^{k-n}(\Omega)$, and so the dual complex

$$
0 \leftarrow H^{*} \Lambda^{0}(\Omega) \stackrel{\delta}{\leftarrow} H^{*} \Lambda^{n}(\Omega) \stackrel{\delta}{\leftarrow} \cdots \stackrel{\delta}{\leftarrow} H^{*} \Lambda^{n}(\Omega) \leftarrow 0
$$

contains the same information as the de Rham complex.

${ }^{1}$ The $L^{2}$ de Rham complex is often written $0 \rightarrow L^{2} \Lambda^{0}(\Omega) \stackrel{\mathrm{d}}{\rightarrow} \cdots \stackrel{\mathrm{d}}{\rightarrow} L^{2} \Lambda^{n}(\Omega) \rightarrow 0$ where the $\mathrm{d}$ are taken as unbounded operators with the $H \Lambda^{k}(\Omega)$ spaces as domains. This is an equivalent notion. 


\section{Boundary traces}

Using the theory of traces in Sobolev space, we find that the trace operator $\operatorname{Tr}: \Lambda^{k}(\Omega) \rightarrow \Lambda^{k}(\partial \Omega)$ extends by continuity to a mapping of $H^{1} \Lambda^{k}(\Omega)$ onto the Sobolev space $H^{1 / 2} \Lambda^{k}(\partial \Omega)$. Of course, the trace cannot be extended to all of $L^{2} \Lambda(\Omega)$. However, we can give a meaning to the trace of $\omega \in H \Lambda(\Omega)$ as follows. Given $\rho \in H^{1 / 2} \Lambda^{k}(\partial \Omega)$, let $\nwarrow \rho \in H^{1 / 2} \Lambda^{n-k-1}(\partial \Omega)$ denote the Hodge star of $\rho$ with respect to the boundary. Then we can find $\eta \in$ $H^{1} \Lambda^{n-k-1}(\Omega)$ with $\operatorname{Tr} \eta=\bar{\star} \rho$ and

$$
\|\eta\|_{H^{1}} \leq c\|\bar{\star} \rho\|_{H^{1 / 2}} \leq c\|\rho\|_{H^{1 / 2}} \text {. }
$$

Now for $\omega \in \Lambda^{k}(\Omega),(2.4)$ gives

$$
\begin{aligned}
\langle\operatorname{Tr} \omega, \rho\rangle & =\int_{\partial \Omega}(\operatorname{Tr} \omega) \wedge \mp \rho=\int_{\partial \Omega} \operatorname{Tr} \omega \wedge \operatorname{Tr} \eta \\
& =\int_{\Omega}\left[\mathrm{d} \omega \wedge \eta+(-1)^{k} \omega \wedge \delta \eta\right] \leq c\|\omega\|_{H \Lambda}\|\eta\|_{H^{1}} \\
& \leq c\|\omega\|_{H \Lambda}\|\rho\|_{H^{1 / 2}} .
\end{aligned}
$$

It follows that we can extend $\operatorname{Tr}$ to a bounded operator on $H \Lambda^{k}(\Omega)$ with values in $H^{-1 / 2} \Lambda^{k}(\partial \Omega)$, the dual of $H^{1 / 2} \Lambda^{k}(\partial \Omega)$. We may then define

$$
\stackrel{\circ}{H} \Lambda^{k}(\Omega)=\left\{\omega \in H \Lambda^{k}(\Omega) \mid \operatorname{Tr} \omega=0\right\} .
$$

If $\omega \in H^{*} \Lambda^{k}(\Omega)$, then $\star \omega \in H \Lambda^{n-k}(\Omega)$, so $\operatorname{Tr}(\star \omega)$ is well-defined. Clearly,

$$
\stackrel{\circ}{H}^{*} \Lambda^{k}(\Omega):=\star \stackrel{\circ}{H} \Lambda^{n-k}(\Omega)=\left\{\omega \in H^{*} \Lambda^{k}(\Omega) \mid \operatorname{Tr}(\star \omega)=0\right\} .
$$

We recall that for $\omega$ smooth, $\operatorname{Tr} \omega$ vanishes at some $x \in \partial \Omega$ if and only if the tangential part of $\omega_{x}$ vanishes, and $\operatorname{Tr}(\star \omega)$ vanishes if and only if the normal part vanishes.

We can use the coderivative operator and the trace operator to characterize the orthogonal complement of the range of $\mathrm{d}$ and $\delta$. From the adjoint equation (2.8), we see that

$$
\begin{aligned}
\left\{\omega \in L^{2} \Lambda^{k}(\Omega) \mid\langle\omega, \mathrm{d} \eta\rangle_{L^{2} \Lambda^{k}}=0 \forall \eta\right. & \left.\in H \Lambda^{k-1}(\Omega)\right\} \\
& =\left\{\omega \in \stackrel{\stackrel{\circ}{H}}{*}^{*}(\Omega) \mid \delta \omega=0\right\} .
\end{aligned}
$$

and

$$
\begin{aligned}
\left\{\omega \in L^{2} \Lambda^{k}(\Omega) \mid\langle\omega, \delta \eta\rangle_{L^{2} \Lambda^{k}}=0 \forall \eta \in\right. & \left.H \Lambda^{k+1}(\Omega)\right\} \\
& =\left\{\omega \in \stackrel{\circ}{H} \Lambda^{k}(\Omega) \mid \mathrm{d} \omega=0\right\} .
\end{aligned}
$$

The first then gives us an expression for the harmonic forms:

$$
\mathfrak{H}^{k}=\left\{\omega \in H \Lambda^{k}(\Omega) \cap \stackrel{\circ}{H}^{*} \Lambda^{k}(\Omega) \mid \mathrm{d} \omega=0, \delta \omega=0\right\} .
$$

In words, the harmonic functions are determined by the differential equations $\mathrm{d} \omega=0$ and $\delta \omega=0$ together with the boundary conditions $\operatorname{Tr} \star \omega=0$. 
Cohomology with boundary conditions

Let $\Lambda^{k}(\Omega)$ denote the subspace of $\Lambda^{k}(\Omega)$ consisting of smooth $k$-forms with compact support. Since pullbacks commute with exterior differentiation, $\operatorname{Tr} \mathrm{d} \omega=\mathrm{d} \operatorname{Tr} \omega$, and so $\mathrm{d} \Lambda^{k}(\Omega) \subset \Lambda^{k+1}(\Omega)$. The de Rham complex with compact support is then

$$
0 \rightarrow \AA^{0}(\Omega) \stackrel{\mathrm{d}}{\rightarrow} \AA^{1}(\Omega) \stackrel{\mathrm{d}}{\rightarrow} \cdots \stackrel{\mathrm{d}}{\rightarrow} \AA^{n}(\Omega) \rightarrow 0 .
$$

Since the closure of $\AA^{k}(\Omega)$ in $H \Lambda^{k}(\Omega)$ is $\stackrel{\circ}{H} \Lambda^{k}(\Omega)$, the $L^{2}$ version of the complex (2.13), with the same cohomology, is

$$
0 \rightarrow \stackrel{\circ}{H} \Lambda^{0}(\Omega) \stackrel{\mathrm{d}}{\rightarrow} \stackrel{\circ}{H} \Lambda^{1}(\Omega) \stackrel{\mathrm{d}}{\rightarrow} \cdots \stackrel{\mathrm{d}}{\rightarrow} \stackrel{\circ}{H} \Lambda^{n}(\Omega) \rightarrow 0 .
$$

The cohomology space is again isomorphic to a space of harmonic forms, in this case,

$$
\stackrel{\circ}{\mathfrak{H}}^{k}(\Omega)=\left\{\omega \in \stackrel{\circ}{H} \Lambda^{k}(\Omega) \mid \mathrm{d} \omega=0,\langle\omega, \mathrm{d} \eta\rangle_{L^{2} \Lambda^{k}}=0 \forall \eta \in \stackrel{\circ}{H} \Lambda^{k-1}\right\} .
$$

In analogy to (2.10), (2.11), and (2.12), we have

$$
\begin{aligned}
& \left\{\omega \in L^{2} \Lambda^{k}(\Omega) \mid\langle\omega, \mathrm{d} \eta\rangle_{L^{2} \Lambda^{k}}=0 \forall \eta \in \stackrel{\circ}{H} \Lambda^{k-1}(\Omega)\right\} \\
& \quad=\left\{\omega \in H^{*} \Lambda^{k}(\Omega) \mid \delta \omega=0\right\}, \\
& \left\{\omega \in L^{2} \Lambda^{k}(\Omega) \mid\langle\omega, \delta \eta\rangle_{L^{2} \Lambda^{k}}=0 \forall \eta \in \stackrel{\circ}{H}_{*} \Lambda^{k+1}(\Omega)\right\} \\
& \quad=\left\{\omega \in H \Lambda^{k}(\Omega) \mid \mathrm{d} \omega=0\right\}, \\
& \stackrel{\circ}{\mathfrak{H}}^{k}=\left\{\omega \in \stackrel{\circ}{H} \Lambda^{k}(\Omega) \cap H^{*} \Lambda^{k}(\Omega) \mid \mathrm{d} \omega=0, \delta \omega=0\right\} .
\end{aligned}
$$

It is a simple untangling of the definitions to see that $\star \mathfrak{H}^{k}(\Omega)=\stackrel{\mathfrak{H}}{ }^{n-k}(\Omega)$. Thus there is an isomorphism between the $k$ th de Rham cohomology space and the $(n-k)$ th cohomology space with boundary conditions. This is called Poincaré duality.

For a contractible domain $\Omega$, the only nonvanishing cohomology space is now that of highest order, and the extended de Rham complex

$$
0 \rightarrow \AA^{0}(\Omega) \stackrel{\mathrm{d}}{\rightarrow} \AA^{1}(\Omega) \stackrel{\mathrm{d}}{\rightarrow} \cdots \stackrel{\mathrm{d}}{\rightarrow} \AA^{n}(\Omega) \stackrel{\int}{\rightarrow} \mathbb{R} \rightarrow 0
$$

is exact.

\section{Homological algebra}

The language of homological algebra was invented to clarify the common algebraic structures behind a variety of constructions in different branches of mathematics, for example the de Rham cohomology of differential geometry and simplicial homology in algebraic topology. Here we introduce some of the basic definitions of homological algebra which will be useful below, 
including cochain maps, cochain projections, and cochain homotopies. More details can be found in many places, e.g., Hilton and Stammbach (1997).

A cochain complex is a sequence of real vector spaces (or more generally modules or Abelian groups, but we will only use vector spaces) and maps:

$$
\cdots \rightarrow V_{k-1} \stackrel{\mathrm{d}_{k-1}}{\longrightarrow} V_{k} \stackrel{\mathrm{d}_{k}}{\longrightarrow} V_{k+1} \rightarrow \cdots
$$

with $\mathrm{d}_{k+1} \circ \mathrm{d}_{k}=0$. Equivalently, we may think of a cochain complex as the graded algebra $V=\bigoplus V_{k}$ equipped with a graded linear operator $\mathrm{d}: V \rightarrow V$ of degree +1 satisfying $\mathrm{d} \circ \mathrm{d}=0$. A chain complex is the same thing except that the indices decrease, and all the definitions below apply mutatis mutandi to chain complexes. The de Rham complex $(\Lambda(\Omega)$, d) and its variants (2.6), (2.13), and (2.14) are examples of cochain complexes, while the dual complex (2.9) and its variants are chain complexes. All the complexes we consider are nonnegative in that $V_{k}=0$ for $k<0$, and bounded in that $V_{n}=0$ for $n$ sufficiently large.

Given a cochain complex $V$, the elements of $\mathcal{N}\left(\mathrm{d}_{k}\right)$ are called the $k$ cocycles and the elements of $\mathcal{R}\left(\mathrm{d}_{k}\right)$ the $k$-coboundaries. The quotient space $H^{k}(V):=\mathcal{N}\left(\mathrm{d}_{k}\right) / \mathcal{R}\left(\mathrm{d}_{k}\right)$ is the $k$ th cohomology space.

Given two cochain complexes $V$ and $V^{\prime}$, a set of maps $f_{k}: V_{k} \rightarrow V_{k}^{\prime}$ satisfying $\mathrm{d}_{k}^{\prime} f_{k}=f_{k+1} \mathrm{~d}_{k}$ (i.e., is a graded linear map $f: V \rightarrow V^{\prime}$ of degree 0 satisfying $\mathrm{d}^{\prime} f=f \mathrm{~d}$ ) is called a cochain map. When $f$ is a cochain map, $f_{k}$ maps $k$-cochains to $k$-cochains and $k$-coboundaries to $k$-coboundaries, and hence induces a map $H^{k}(f): H^{k}(V) \rightarrow H^{k}\left(V^{\prime}\right)$.

If $V$ is a cochain complex and $V^{\prime}$ a subcomplex (i.e., $V_{k}^{\prime} \subset V_{k}$ and $\mathrm{d} V_{k}^{\prime} \subset$ $\left.V_{k+1}^{\prime}\right)$, then the inclusion $i: V^{\prime} \rightarrow V$ is a cochain map and so induces a map of cohomology $H^{k}\left(V^{\prime}\right) \rightarrow H^{k}(V)$. If there exists a cochain projection of $V$ onto $V^{\prime}$, i.e., a cochain map $\pi$ such that $\pi_{k}: V_{k} \rightarrow V_{k}^{\prime}$ restricts to the identity on $V_{k}^{\prime}$, then $\pi \circ i=\operatorname{id}_{V^{\prime}}$, so $H^{k}(\pi) \circ H^{k}(i)=\operatorname{id}_{H^{k}\left(V^{\prime}\right)}$. We conclude that in this case $H^{k}(i)$ is injective and $H^{k}(\pi)$ is surjective. In particular, if one of the cohomology spaces $H^{k}(V)$ vanishes, then so does $H^{k}\left(V^{\prime}\right)$. We shall use this property frequently.

Given a cochain map $f: V \rightarrow V^{\prime}$, a graded linear map $\kappa: V \rightarrow V^{\prime}$ of degree -1 (i.e., a sequence of maps $\kappa_{k}: V_{k} \rightarrow V_{k-1}^{\prime}$ ) is called a contracting cochain homotopy for $f$, if

$$
f_{k}=\mathrm{d}_{k-1}^{\prime} \kappa_{k}+\kappa_{k+1} \mathrm{~d}_{k}: V_{k} \rightarrow V_{k+1}
$$

If there exists a contracting homotopy for $f$, then $f$ induces the zero map on cohomology (since if $z$ is a $k$-cocycle, then $f z=\mathrm{d}^{\prime} \kappa z+\kappa \mathrm{d} z=\mathrm{d}^{\prime} \kappa z$ is a $k$-coboundary). In particular, if the identity cochain map on $V$ admits a contracting homotopy, then the cohomology of $V$ vanishes. We shall see an example of this when we discuss the Koszul complex (Theorem 3.1). 
Cycles and boundaries of the de Rham complex

We have four variants of the $L^{2}$ de Rham complex: (2.6), the dual version (2.9), and versions each of these incorporating boundary conditions, namely (2.14) and the dual analogue. For each of these we have the corresponding spaces of cycles and boundaries (for brevity we use the term cycles and boundaries to refer as well to cocycles and coboundaries). We denote these by

$$
\begin{array}{ll}
\mathfrak{Z}^{k}=\left\{\omega \in H \Lambda^{k}(\Omega) \mid \mathrm{d} \omega=0\right\}, & \mathfrak{Z}^{* k}=\left\{\omega \in H^{*} \Lambda^{k}(\Omega) \mid \delta \omega=0\right\}, \\
\mathfrak{\mathfrak { Z }}^{k}=\left\{\omega \in \stackrel{\circ}{H} \Lambda^{k}(\Omega) \mid \mathrm{d} \omega=0\right\}, & \mathfrak{Z}^{* k}=\left\{\omega \in \stackrel{\circ}{H}^{*} \Lambda^{k}(\Omega) \mid \delta \omega=0\right\},
\end{array}
$$

and

$$
\begin{array}{ll}
\mathfrak{B}^{k}=\mathrm{d} H \Lambda^{k-1}(\Omega), & \mathfrak{B}^{* k}=\delta \Lambda^{k+1}(\Omega), \\
\stackrel{\circ}{\mathfrak{B}}^{k}=\mathrm{d} \stackrel{\circ}{H} \Lambda^{k-1}(\Omega), & \stackrel{\circ}{\mathfrak{B}}^{* k}=\delta \AA^{k+1}(\Omega) .
\end{array}
$$

Each of the spaces of cycles is obviously closed in the space $H \Lambda^{k}(\Omega)$ or $H^{*} \Lambda^{k}(\Omega)$, as appropriate. Each is closed in $L^{2} \Lambda^{k}(\Omega)$ as well. For example, we show that $\mathfrak{Z}^{k}$ is $L^{2}$ closed. Suppose that $\omega_{n} \in \mathfrak{Z}^{k}$ and $\omega_{n}$ converges to some $\omega$ in $L^{2} \Lambda^{k}(\Omega)$. We must show that $\omega \in \stackrel{\circ}{H} \Lambda^{k}(\Omega)$ and $\mathrm{d} \omega=0$. Now convergence of $\omega_{n}$ to $\omega$ in $L^{2}$ implies convergence of $\mathrm{d} \omega_{n}$ to $\mathrm{d} \omega$ in $H^{-1}$. Since $\mathrm{d} \omega_{n}=0$, we conclude that $\mathrm{d} \omega=0$, so $\omega \in H \Lambda^{k}(\Omega)$. Since we have convergence of both $\omega_{n}$ and $\mathrm{d} \omega_{n}$, we have that $\omega_{n}$ converges to $\omega$ in $H \Lambda^{k}(\Omega)$, and so $\omega$ must belong to $\mathfrak{Z}^{k}$.

The spaces of boundaries are all closed in $L^{2} \Lambda^{k}(\Omega)$ as well. This will follow from Poincaré's inequality, in the next subsubsection.

For any of these subspaces of $L^{2} \Lambda^{k}(\Omega)$, we use the superscript $\perp$ to denote its orthogonal complement in that space. Note that the orthogonal complement of $\mathfrak{Z}^{k}$ in $H \Lambda^{k}(\Omega)$ is just $H \Lambda^{k}(\Omega) \cap \mathfrak{Z}^{k \perp}$, and a similar relationship holds for all the other spaces. Equation (2.10) can be rewritten as $\mathfrak{B}^{k \perp}=\mathfrak{Z}^{* k \perp}$. In fact, it is easy to verify using (2.8) that

$$
\begin{array}{ll}
\mathfrak{Z}^{k \perp} \subset \mathfrak{B}^{k \perp}=\mathfrak{\mathfrak { Z }}^{* k}, & \mathfrak{Z}^{* k \perp} \subset \mathfrak{B}^{* k \perp}=\mathfrak{\mathfrak { Z }}^{k}, \\
\mathfrak{\mathfrak { Z }}^{k \perp} \subset \mathfrak{\mathfrak { B }}^{k \perp}=\mathfrak{Z}^{* k}, & \mathfrak{Z}^{* k \perp} \subset \mathfrak{\mathfrak { B }}^{* k \perp}=\mathfrak{Z}^{k} .
\end{array}
$$

Compactness and Poincaré's inequality

If $\Omega$ is a smoothly bounded oriented Riemannian manifold with boundary, then the space $H \Lambda^{k}(\Omega) \cap \stackrel{\circ}{H}^{*} \Lambda(\Omega)$ is a subspace of $H^{1} \Lambda^{k}(\Omega)$, and there holds the estimate (Gaffney 1951)

$$
\|\omega\|_{H^{1}} \leq c(\|\mathrm{~d} \omega\|+\|\delta \omega\|+\|\omega\|) .
$$

(A similar result holds for $\stackrel{\circ}{H} \Lambda^{k}(\Omega) \cap H^{*} \Lambda^{k}(\Omega)$.) We can then apply Rellich's lemma to conclude that $H \Lambda^{k}(\Omega) \cap \stackrel{\circ}{H}^{*} \Lambda(\Omega)$ is compactly embedded in $L^{2} \Lambda^{k}(\Omega)$. 
For polyhedral $\Omega$, the space $H \Lambda^{k}(\Omega) \cap \stackrel{\circ}{H}^{*} \Lambda(\Omega)$ may not be embedded in $H^{1} \Lambda^{k}(\Omega)$. However the following theorem, which is proved in Picard (1984) for manifolds with Lipschitz boundary, states that the compact embedding into $L^{2}$ still holds.

Theorem 2.1. The embeddings of $H \Lambda^{k}(\Omega) \cap \stackrel{\circ}{H}^{*} \Lambda^{k}(\Omega)$ and $\stackrel{\circ}{H} \Lambda^{k}(\Omega) \cap$ $H^{*} \Lambda^{k}(\Omega)$ into $L^{2} \Lambda^{k}(\Omega)$ are compact.

From this we obtain Poincaré's inequality by a standard compactness argument.

Theorem 2.2. There exists a constant $c$ such that

$$
\|\omega\| \leq c(\|\mathrm{~d} \omega\|+\|\delta \omega\|)
$$

for $\omega \in H \Lambda^{k}(\Omega) \cap \stackrel{\circ}{H}^{*} \Lambda^{k}(\Omega) \cap \mathfrak{H}^{k \perp}$ or $\stackrel{\circ}{H} \Lambda^{k}(\Omega) \cap H^{*} \Lambda^{k}(\Omega) \cap \stackrel{\circ}{\mathfrak{H}}^{k \perp}$.

Proof. We will give the proof for $H \Lambda^{k}(\Omega) \cap \stackrel{\circ}{H}^{*} \Lambda^{k}(\Omega) \cap \mathfrak{H}^{k \perp}$, the other case being similar. If the result were not true, we could find a sequence of $\omega_{n} \in$ $H \Lambda^{k}(\Omega) \cap \stackrel{\circ}{H}^{*} \Lambda^{k}(\Omega) \cap \mathfrak{H}^{k \perp}$ such that $\left\|\omega_{n}\right\|=1$ while $\mathrm{d} \omega_{n} \rightarrow 0$ and $\delta \omega_{n} \rightarrow 0$ in $L^{2}$. The sequence is certainly bounded in $H \Lambda^{k}(\Omega) \cap \stackrel{\circ}{H}^{*} \Lambda^{k}(\Omega)$, and, so precompact in $L^{2} \Lambda^{k}(\Omega)$ by Theorem 2.1. Passing to a subsequence, we have $\omega_{n}$ converges in $L^{2} \Lambda^{k}(\Omega)$ to some $\omega \in L^{2} \Lambda^{k}(\Omega)$. Clearly $\mathrm{d} \omega=0, \delta \omega=0$, and the convergence holds in $H \Lambda^{k}(\Omega)$. Thus $\omega \in H \Lambda^{k}(\Omega) \cap \stackrel{\circ}{H}^{*} \Lambda^{k}(\Omega)$ and so $\omega \in \mathfrak{H}^{k}$ by $(2.12)$, but also in $\mathfrak{H}^{k \perp}$, and so $\omega=0$. But $\|\omega\|=\lim \left\|\omega_{n}\right\|=1$, which is a contradiction.

A special, but very useful, case is if $\omega \in \mathfrak{Z}^{k \perp}$. Then $\omega \in H \Lambda^{k}(\Omega) \cap$ $\stackrel{\circ}{H}^{*} \Lambda^{k}(\Omega) \cap \mathfrak{H}^{k \perp}$, so

$$
\|\omega\| \leq c\|\mathrm{~d} \omega\|, \quad \omega \in \mathfrak{Z}^{k \perp} .
$$

Of course the analogous result for the coderivative holds as well.

Theorem 2.3. The spaces of boundaries, $\mathfrak{B}^{k}, \mathfrak{B}^{* k}, \stackrel{\circ}{\mathfrak{B}}^{k}$, and $\mathfrak{\mathfrak { B }}^{* k}$, are closed in $L^{2} \Lambda^{k}(\Omega)$.

Proof. We prove that $\stackrel{\circ}{\mathfrak{B}}^{k}$ is closed, the other cases being similar. Suppose $\omega_{n} \in \stackrel{\circ}{\mathfrak{B}}^{k}$ and $\omega_{n} \rightarrow \omega$ in $L^{2} \Lambda^{k}(\Omega)$. There exist $\eta_{n} \in \mathfrak{Z}^{k \perp}$ with $\omega_{n}=$ $\mathrm{d} \eta_{n}$. From the fact that the sequence $\mathrm{d} \eta_{n}$ is convergent in $L^{2} \Lambda^{k}(\Omega)$ and Poincaré's inequality, we find that $\eta_{n}$ is Cauchy with respect to the $L^{2}$-norm and so $\eta_{n}$ converges to some $\eta \in L^{2}$. Necessarily $\mathrm{d} \eta=\omega$, so $\eta_{n}$ converges to $\eta$ in $H \Lambda^{k}(\Omega)$, so $\eta \in \stackrel{\circ}{H} \Lambda^{k}(\Omega)$ and $\omega=\mathrm{d} \eta \in \dot{\mathfrak{B}}^{k}$.

\section{Hodge decomposition}

At this point, the Hodge decomposition (or really two Hodge decompositions, with different boundary conditions), is just a matter of gathering results. 
From the first equality in (2.15), we have an orthogonal decomposition

$$
L^{2} \Lambda^{k}(\Omega)=\mathfrak{B}^{k} \oplus \dot{\mathfrak{Z}}^{* k} .
$$

But $\dot{\mathfrak{B}}^{* k}$ is a closed subspace of $\dot{\mathfrak{Z}}^{* k}$ whose orthogonal complement is, by the second equality in (2.16),

$$
\stackrel{\mathfrak{Z}}{ }^{* k} \cap \mathfrak{Z}^{k}=\mathfrak{H}^{k} .
$$

Thus

$$
L^{2} \Lambda^{k}(\Omega)=\mathfrak{B}^{k} \oplus \mathfrak{H}^{k} \oplus \stackrel{\circ}{\mathfrak{B}}^{* k},
$$

which is the first Hodge decomposition. The second follows analogously. The following equation summarizes both, together with the relations of the various spaces discussed:

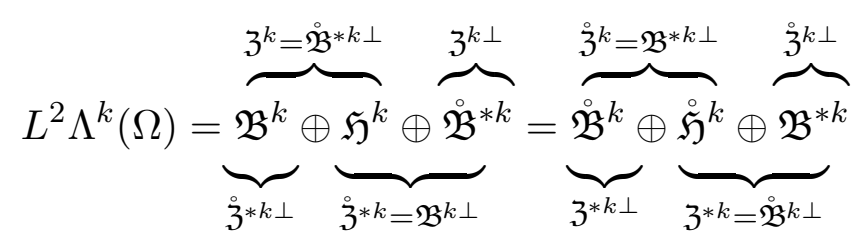

The Hodge Laplacian

The differential operator $\delta \mathrm{d}+\mathrm{d} \delta$ maps $k$-forms to $k$-forms. It is called the Hodge Laplacian. Here we briefly consider boundary value problems for the Hodge Laplacian, taking a variational approach.

Consider first the problem of minimizing the quadratic functional

$$
\mathcal{J}(u)=\frac{1}{2}\langle\mathrm{~d} u, \mathrm{~d} u\rangle+\frac{1}{2}\langle\delta u, \delta u\rangle-\langle f, u\rangle
$$

over $u \in H \Lambda^{k}(\Omega) \cap \stackrel{\circ}{H}^{*} \Lambda^{k}(\Omega)$ where $f \in L^{2} \Lambda^{k}(\Omega)$ is given. Here we run into a problem if the space of harmonic forms $\mathfrak{H}^{k}$ is not 0 . If the $L^{2}$ projection $P_{\mathfrak{H}^{k}} f$ is not zero, then $\mathcal{J}$ admits no minimum, since we could take $u=c P_{\mathfrak{H}^{k}} f$ for $c$ arbitrarily large. If we insist that $f$ be orthogonal to $\mathfrak{H}^{k}$, then there exists a minimizer, but it is not unique, because the addition of a harmonic function to $u$ will not change $\mathcal{J}(u)$. To avoid this difficulty we can work in the orthogonal complement of $\mathfrak{H}^{k}$. That is, we assume that $f \in \mathfrak{H}^{k \perp}$, and seek $u \in X:=H \Lambda^{k}(\Omega) \cap \stackrel{\circ}{H}^{*} \Lambda^{k}(\Omega) \cap \mathfrak{H}^{k \perp}$ minimizing $\mathcal{J}(u)$. Now $X$ is a Hilbert space with norm

$$
\|u\|_{X}=\|u\|_{H \Lambda}+\|u\|_{H^{*} \Lambda} .
$$

By Poincaré's inequality, Theorem 2.2, the square root of the quadratic part of $\mathcal{J}(u)$ defines an equivalent norm:

$$
\|u\|_{X} \approx\|\mathrm{d} u\|+\|\delta u\|, \quad u \in X .
$$

Therefore it is easy to prove that there exists a unique minimizer $u \in X$ 
which satisfies the Euler-Lagrange equations

$$
\langle\mathrm{d} u, \mathrm{~d} v\rangle+\langle\delta u, \delta v\rangle=\langle f, v\rangle, \quad v \in X .
$$

In fact, these equations are satisfied for all $v \in H \Lambda^{k}(\Omega) \cap \stackrel{\circ}{H}^{*} \Lambda^{k}(\Omega)$, since they are trivially satisfied for $v \in \mathfrak{H}^{k}$ (using the fact that $f \perp \mathfrak{H}^{k}$ ). Thus, in view of (2.8), the solution $u$ satisfies the differential equation $(\delta \mathrm{d}+\mathrm{d} \delta) u=f$ and also the boundary condition $\operatorname{Tr}(\star \mathrm{d} u)=0$ in a weak sense. We have also imposed the boundary condition $\operatorname{Tr}(\star u)=0$ and orthogonality to $\mathfrak{H}^{k}$, i.e., we have well-posedness, in an appropriate sense, of the following boundary value problem: given $f \in L^{2} \Lambda^{k}(\Omega)$ orthogonal to $\mathfrak{H}^{k}$, find $u$ such that

$$
(\delta \mathrm{d}+\mathrm{d} \delta) u=f \text { in } \Omega, \quad \operatorname{Tr}(\star u)=0, \operatorname{Tr}(\star \mathrm{d} u)=0 \text { on } \partial \Omega, \quad u \perp \mathfrak{H}^{k} .
$$

A slightly different way to handle the harmonic functions, which we shall follow when we discuss mixed formulations in Section 7, is to impose the orthogonality condition via a Lagrange multiplier $p \in \mathfrak{H}^{k}$. Then the weak problem is to find $u \in H \Lambda^{k}(\Omega) \cap \stackrel{\circ}{H}^{*} \Lambda^{k}(\Omega)$ and $p \in \mathfrak{H}^{k}$ such that

$$
\begin{aligned}
\langle\mathrm{d} u, \mathrm{~d} v\rangle+\langle\delta u, \delta v\rangle+\langle p, v\rangle & =\langle f, v\rangle, & & v \in H \Lambda^{k}(\Omega) \cap \stackrel{\circ}{H}^{*} \Lambda^{k}(\Omega), \\
\langle u, q\rangle & =0, & & q \mathfrak{H}^{k} .
\end{aligned}
$$

The differential equation is now $(\delta \mathrm{d}+\mathrm{d} \delta) u+p=f$, which has a solution for any $f$. We get $p=P_{\mathfrak{H}^{k} f}$ and so $p$ vanishes if $f \perp \mathfrak{H}^{k}$.

Returning to the first approach, we could have also chosen the space $X$ to be $\stackrel{\circ}{H} \Lambda^{k}(\Omega) \cap H^{*} \Lambda^{k}(\Omega) \cap \dot{\mathfrak{H}}^{k \perp}$. In this case we obtain the same differential equation, but the weakly imposed boundary condition $\operatorname{Tr}(\delta u)=0$ and the strongly imposed boundary condition $\operatorname{Tr}(u)=0$, and the orthogonality assumed on $f$ and imposed on $u$ is to $\mathfrak{H}^{k}$ rather than to $\mathfrak{H}^{k}$.

The finite element solution of this problem might seem straightforward, at least when the harmonic functions vanish, but for some domains it most definitely is not. Take, for instance, the case where $\Omega$ is a contractible but nonconvex polyhedron in three dimensions and the form degree $k=1$ or 2. It is shown in Costabel (1991) that the norm on the space $X=$ $H \Lambda^{k}(\Omega) \cap \stackrel{\circ}{H}^{*} \Lambda^{k}(\Omega)$ is equivalent to the $H^{1} \Lambda^{k}(\Omega)$-norm when restricted to $X^{1}:=H^{1} \Lambda^{k}(\Omega) \cap H^{*} \Lambda^{k}(\Omega)$, but that $X^{1}$ is a closed infinite-codimensional subspace of $X$, and that except for very nongeneric data, the solution $u$ to the Hodge Laplacian problem will belong to $X$ but not to $X^{1}$. Now if we triangulate the domain and use piecewise smooth (e.g., finite element) forms in $X$ to approximate the solution, we will not converge to the solution. For a piecewise smooth form which belongs to $H \Lambda^{k}(\Omega) \cap H^{*} \Lambda^{k}(\Omega)$ always belongs to $H^{1}$, and so if it belongs to $X$, it belongs to $X^{1}$. Thus our approximate solutions will remain in a closed subspace which does not contain the exact solution, and so cannot converge to it. 
The mixed formulation we present in Section 7 will not suffer from this (very serious) defect.

\subsection{Exterior calculus on $\mathbb{R}^{n}$}

\section{Global coordinates}

Suppose that $\Omega$ is an open subset of $\mathbb{R}^{n}$. Then we have the global coordinate functions $x_{i}, i=1, \ldots, n$. Moreover, each of the tangent spaces $T_{x} \Omega$ may be identified naturally with $\mathbb{R}^{n}$, and so with each other. This simplifies matters greatly, especially from the computational point of view. Note that the exterior derivative of the coordinate function $x_{i}$ is the functional that takes a vector $v \in \mathbb{R}^{n}$ to its $i$ th component $v_{i} \in \mathbb{R}$. This explains the notation $\mathrm{d} x_{i}$ introduced for this functional in Section 2.1.

A general element of $\Lambda^{k}(\Omega)$ may be written

$$
\omega_{x}=\sum_{1 \leq \sigma(1)<\cdots<\sigma(k) \leq n} a_{\sigma} \mathrm{d} x_{\sigma(1)} \wedge \cdots \wedge \mathrm{d} x_{\sigma(k)},
$$

where the $a_{\sigma} \in C^{\infty}(\Omega)$. If we allow instead $a_{\sigma} \in C^{p}(\Omega), a_{\sigma} \in L^{2}(\Omega)$, $a_{\sigma} \in H^{s}(\Omega)$, etc., we obtain the spaces $C^{p} \Lambda(\Omega), L^{2} \Lambda(\Omega), H^{s} \Lambda(\Omega)$, etc.

The volume form is simply $\mathrm{d} x_{1} \wedge \cdots \wedge \mathrm{d} x_{n}$.

In terms of the global coordinates, the exterior derivative also has a simple expression:

$$
\mathrm{d} \sum a_{\sigma} \mathrm{d} x_{\sigma(1)} \wedge \cdots \wedge \mathrm{d} x_{\sigma(k)}=\sum_{\sigma} \sum_{i=1}^{n} \frac{\partial a_{\sigma}}{\partial x_{i}} \mathrm{~d} x_{i} \wedge \mathrm{d} x_{\sigma(1)} \wedge \cdots \wedge \mathrm{d} x_{\sigma(k)} .
$$

\section{Proxy fields}

Based on the identification of $\mathrm{Alt}^{0} \mathbb{R}^{n}$ and $\mathrm{Alt}^{n} \mathbb{R}^{n}$ with $\mathbb{R}$ and of $\mathrm{Alt}^{1} \mathbb{R}^{n}$ and $\mathrm{Alt}^{n-1} \mathbb{R}^{n}$ with $\mathbb{R}^{n}$, we may identify each 0 -form and $n$-form with a scalar-valued function and, for $n>1$, each 1 -form and $(n-1)$-form with a vector field. The associated fields are called proxy fields for the forms. For $n=2$ we have two different identifications of $\Lambda^{1}(\Omega)$ with $C^{\infty}\left(\Omega ; \mathbb{R}^{2}\right)$, i.e., two ways to associate a proxy field to a 1-form.

Interpreted in terms of the proxy fields, the exterior derivative operators $\mathrm{d}: \Lambda^{0}(\Omega) \rightarrow \Lambda^{1}(\Omega)$ and $\mathrm{d}: \Lambda^{n-1}(\Omega) \rightarrow \Lambda^{n}(\Omega)$ become grad $: C^{\infty}(\Omega) \rightarrow$ $C^{\infty}\left(\Omega ; \mathbb{R}^{n}\right)$ and $\operatorname{div}: C^{\infty}\left(\Omega ; \mathbb{R}^{n}\right) \rightarrow C^{\infty}(\Omega)$, respectively.

Table 2.2 summarizes correspondences between differential forms and their proxy fields in the case $\Omega \subset \mathbb{R}^{3}$ (The last column refers to the Koszul differential, introduced in the next section.)

For $\Omega \subset \mathbb{R}^{3}$, the de Rham complex becomes

$$
0 \rightarrow C^{\infty}(\Omega) \stackrel{\text { grad }}{\longrightarrow} C^{\infty}\left(\Omega ; \mathbb{R}^{3}\right) \stackrel{\text { curl }}{\longrightarrow} C^{\infty}\left(\Omega ; \mathbb{R}^{3}\right) \stackrel{\text { div }}{\longrightarrow} C^{\infty}(\Omega) \rightarrow 0,
$$


Table 2.2. Correspondences between a differential forms $\omega$ on $\Omega \subset \mathbb{R}^{3}$ and scalar/vector fields $w$ on $\Omega$. In the integrals, $f$ denotes a submanifold of dimension $k$, and $\mathcal{H}_{k}$ denotes the $k$-dimensional Hausdorff measure (Lebesgue measure for $k=3$ ). The unit tangent $t$ for $k=1$ and unit normal $n$ for $k=2$ are determined by the orientation of $f$.

\begin{tabular}{c|ccccc}
\hline \hline$k$ & $\Lambda^{k}(\Omega)$ & $H \Lambda^{k}(\Omega)$ & $\mathrm{d} \omega$ & $\int_{f} \omega$ & $\kappa \omega$ \\
\hline 0 & $C^{\infty}(\Omega)$ & $H^{1}(\Omega)$ & $\operatorname{grad} w$ & $w(f)$ & 0 \\
1 & $C^{\infty}\left(\Omega ; \mathbb{R}^{3}\right)$ & $H\left(\operatorname{curl}, \Omega ; \mathbb{R}^{3}\right)$ & $\operatorname{curl} w$ & $\int_{f} w \cdot t \mathrm{~d} \mathcal{H}_{1}$ & $x \mapsto x \cdot w(x)$ \\
2 & $C^{\infty}\left(\Omega ; \mathbb{R}^{3}\right)$ & $H\left(\operatorname{div}, \Omega ; \mathbb{R}^{3}\right)$ & $\operatorname{div} w$ & $\int_{f} w \cdot n \mathrm{~d} \mathcal{H}_{2}$ & $x \mapsto x \times w(x)$ \\
3 & $C^{\infty}(\Omega)$ & $L^{2}(\Omega)$ & 0 & $\int_{f} w \mathrm{~d} \mathcal{H}_{3}$ & $x \rightarrow x w(x)$ \\
\hline
\end{tabular}

and the $L^{2}$ de Rham complex

$$
0 \rightarrow H^{1}(\Omega) \stackrel{\text { grad }}{\longrightarrow} H\left(\operatorname{curl}, \Omega ; \mathbb{R}^{3}\right) \stackrel{\text { curl }}{\longrightarrow} H\left(\operatorname{div}, \Omega ; \mathbb{R}^{3}\right) \stackrel{\text { div }}{\longrightarrow} L^{2}(\Omega) \rightarrow 0 .
$$

For $\Omega \subset \mathbb{R}^{2}$, the de Rham complex becomes

$$
0 \rightarrow C^{\infty}(\Omega) \stackrel{\text { grad }}{\longrightarrow} C^{\infty}\left(\Omega ; \mathbb{R}^{2}\right) \stackrel{\text { rot }}{\longrightarrow} C^{\infty}(\Omega) \rightarrow 0,
$$

or

$$
0 \rightarrow C^{\infty}(\Omega) \stackrel{\text { curl }}{\longrightarrow} C^{\infty}\left(\Omega ; \mathbb{R}^{2}\right) \stackrel{\text { div }}{\longrightarrow} C^{\infty}(\Omega) \rightarrow 0,
$$

depending on which of the two identification we choose for $\Lambda^{1}\left(\mathbb{R}^{2}\right)$.

For $\Omega$ any bounded domain in $\mathbb{R}^{n}$ with Lipschitz boundary, it is known that the divergence operator maps $H^{1}\left(\Omega ; \mathbb{R}^{n}\right)$ onto $L^{2}(\Omega)$ and $\stackrel{\circ}{H}^{1}\left(\Omega ; \mathbb{R}^{n}\right)$ onto the orthogonal complement of the constants in $L^{2}(\Omega)$ (see Girault and Raviart (1986, Corollary 2.4)). Since we will need this result in the final section of the paper, we state it here in the language of differential forms.

Theorem 2.4. Let $\Omega$ be a bounded domain in $\mathbb{R}^{n}$ with a Lipschitz boundary. Then, for all $\mu \in L^{2} \Lambda^{n}(\Omega)$ there exists $\eta \in H^{1} \Lambda^{n-1}(\Omega)$ satisfying $\mathrm{d} \eta=\mu$. If, in addition, $\int_{\Omega} \mu=0$, then we can choose $\eta \in \stackrel{\circ}{H}^{1} \Lambda^{n-1}(\Omega)$.

\section{Polynomial differential forms and the Koszul complex}

In this section we consider spaces of polynomial differential forms, which lead to a variety of subcomplexes of the de Rham complex. These will be used in later sections to construct finite element spaces of differential forms. A key tool will be the Koszul differential and the associated Koszul complex. The material in the first two subsections can be extracted from the literature, to which some references are given, but the goals, context, and level of generality are often quite different from ours, so we intend the presentation 
here to be self-contained. In Section 3.3 we introduce the spaces $\mathcal{P}_{r}^{-} \Lambda^{k}$, which will be of great importance later. (We introduced these spaces under the name $\mathcal{P}_{r-1}^{+} \Lambda^{k}$ in Arnold et al. (2006b), but have changed the indexing in order to have the graded multiplication property (3.16).) In Section 3.4 we determine all finite-dimensional spaces of polynomial differential forms which are invariant under affine transformations. To the best of our knowledge, this result is new. In the following subsection, we exhibit a wide variety of polynomial subcomplexes of the de Rham complex (essentially $2^{n-1}$ of them associated to each polynomial degree). These will lead to finite element de Rham subcomplexes in the following sections. Some of these have appeared in the literature previously, but the systematic derivation of all of them first appeared in Arnold et al. (2006b).

\subsection{Polynomial differential forms}

Let $\mathcal{P}_{r}\left(\mathbb{R}^{n}\right)$ and $\mathcal{H}_{r}\left(\mathbb{R}^{n}\right)$ denote the spaces of polynomials in $n$ variables of degree at most $r$ and of homogeneous polynomial functions of degree $r$, respectively. We interpret these spaces to be the zero space if $r<0$. The space of all polynomial functions is $\mathcal{P}\left(\mathbb{R}^{n}\right)=\bigoplus_{r=0}^{\infty} \mathcal{H}_{r}\left(\mathbb{R}^{n}\right)$, a commutative graded algebra. We can then define spaces of polynomial differential forms, $\mathcal{P}_{r} \Lambda^{k}\left(\mathbb{R}^{n}\right), \mathcal{H}_{r} \Lambda^{k}\left(\mathbb{R}^{n}\right)$, etc. For brevity, we will at times suppress $\mathbb{R}^{n}$ from the notation and write simply $\mathcal{P}_{r}, \mathcal{H}_{r}, \mathcal{P}_{r} \Lambda^{k}$, etc.

We note for future reference that

$$
\begin{aligned}
\operatorname{dim} \mathcal{P}_{r} \Lambda^{k}\left(\mathbb{R}^{n}\right) & =\operatorname{dim} \mathcal{P}_{r}\left(\mathbb{R}^{n}\right) \cdot \operatorname{dim} \mathrm{Alt}^{k} \mathbb{R}^{n} \\
& =\left(\begin{array}{c}
n+r \\
n
\end{array}\right)\left(\begin{array}{l}
n \\
k
\end{array}\right)=\left(\begin{array}{c}
r+k \\
r
\end{array}\right)\left(\begin{array}{l}
n+r \\
n-k
\end{array}\right),
\end{aligned}
$$

and $\operatorname{dim} \mathcal{H}_{r} \Lambda^{k}\left(\mathbb{R}^{n}\right)=\operatorname{dim} \mathcal{P}_{r} \Lambda^{k}\left(\mathbb{R}^{n-1}\right)$.

The space of polynomial differential forms

$$
\mathcal{P} \Lambda=\bigoplus_{r=0}^{\infty} \bigoplus_{k=0}^{n} \mathcal{H}_{r} \Lambda^{k}
$$

is called the Koszul algebra (Guillemin and Sternberg 1999, Chapter 3.1).

For each polynomial degree $r \geq 0$ we get a homogeneous polynomial subcomplex of the de Rham complex:

$$
0 \rightarrow \mathcal{H}_{r} \Lambda^{0} \stackrel{\mathrm{d}}{\longrightarrow} \mathcal{H}_{r-1} \Lambda^{1} \stackrel{\mathrm{d}}{\longrightarrow} \cdots \stackrel{\mathrm{d}}{\longrightarrow} \mathcal{H}_{r-n} \Lambda^{n} \rightarrow 0 .
$$

We shall verify below the exactness of this sequence. More precisely, the cohomology vanishes if $r>0$ and also for $r=0$ except in the lowest degree, where the cohomology space is $\mathbb{R}$ (reflecting the fact that the constants are killed by the gradient).

Taking the direct sum of the homogeneous polynomial de Rham com- 
plexes over all polynomial degrees gives the polynomial de Rham complex:

$$
0 \rightarrow \mathcal{P}_{r} \Lambda^{0} \stackrel{\mathrm{d}}{\longrightarrow} \mathcal{P}_{r-1} \Lambda^{1} \stackrel{\mathrm{d}}{\longrightarrow} \cdots \stackrel{\mathrm{d}}{\longrightarrow} \mathcal{P}_{r-n} \Lambda^{n} \rightarrow 0
$$

for which the cohomology space is $\mathbb{R}$ in the lowest degree, and vanishes otherwise.

It is easy to see that if $\phi: \mathbb{R}^{n} \rightarrow \mathbb{R}^{n}$ is a linear map, then

$$
\phi^{*}\left(\mathcal{H}_{r} \Lambda^{k}\right) \subset \mathcal{H}_{r} \Lambda^{k}, \quad \phi^{*}\left(\mathcal{P}_{r} \Lambda^{k}\right) \subset \mathcal{P}_{r} \Lambda^{k},
$$

and if $\phi: \mathbb{R}^{n} \rightarrow \mathbb{R}^{n}$ is an affine map, then

$$
\phi^{*}\left(\mathcal{P}_{r} \Lambda^{k}\right) \subset \mathcal{P}_{r} \Lambda^{k}
$$

\subsection{The Koszul complex}

Let $x \in \mathbb{R}^{n}$. Since there is a natural identification of $\mathbb{R}^{n}$ with the tangent space $T_{0} \mathbb{R}^{n}$ at the origin, there is a vector in $T_{0} \mathbb{R}^{n}$ corresponding to $x$. (The origin is chosen for convenience here, but we could use any other point instead.) Then translation map $y \mapsto y+x$ induces an isomorphism from $T_{0} \mathbb{R}^{n}$ to $T_{x} \mathbb{R}^{n}$, and so there is an element $X(x) \in T_{x} \mathbb{R}^{n}$ corresponding to $x$. (Essentially $X(x)$ is the vector based at $x$ which points opposite to the origin, and whose length is $|x|$. The interior product with the vector field $X, \kappa:=\lrcorner X$, maps $\Lambda^{k}\left(\mathbb{R}^{n}\right)$ to $\Lambda^{k-1}\left(\mathbb{R}^{n}\right)$ by the formula

$$
(\kappa \omega)_{x}\left(v_{1}, \ldots, v_{k-1}\right)=\omega_{x}\left(X(x), v_{1}, \ldots, v_{k-1}\right) .
$$

From the similar properties for the interior product of algebraic forms, we have that

$$
\kappa \circ \kappa=0
$$

and

$$
\kappa(\omega \wedge \eta)=(\kappa \omega) \wedge \eta+(-1)^{k} \omega \wedge(\kappa \eta), \quad \omega \in \Lambda^{k}, \eta \in \Lambda^{l} .
$$

In terms of coordinates, if $\omega_{x}=\sum_{\sigma} a_{\sigma}(x) \mathrm{d} x_{\sigma(1)} \wedge \cdots \wedge \mathrm{d} x_{\sigma(k)}$, then

$$
(\kappa \omega)_{x}=\sum_{\sigma} \sum_{i=1}^{k}(-1)^{i+1} a_{\sigma}(x) x_{\sigma(i)} \mathrm{d} x_{\sigma(1)} \wedge \cdots \wedge \widehat{\mathrm{d} x}_{\sigma(i)} \wedge \cdots \wedge \mathrm{d} x_{\sigma(k)} .
$$

Note that $\kappa$ maps $\mathcal{H}_{r} \Lambda^{k}$ to $\mathcal{H}_{r+1} \Lambda^{k-1}$, i.e., $\kappa$ increases polynomial degree and decreases form degree, the exact opposite of the exterior derivative $d$.

Another useful formula is the pullback of $\kappa \omega$ under a linear or affine map. First suppose that $\phi: \mathbb{R}^{n} \rightarrow \mathbb{R}^{n}$ is linear. Then if $\omega$ is a $k$-form on $\mathbb{R}^{n}$, we have

$$
\phi^{*} \kappa \omega=\kappa \phi^{*} \omega
$$


Indeed,

$$
\begin{aligned}
\left(\phi^{*} \kappa \omega\right)_{x}\left(v_{1}, \ldots, v_{k-1}\right) & =(\kappa \omega)_{\phi x}\left(\phi v_{1}, \ldots, \phi v_{k-1}\right)=\omega_{\phi x}\left(\phi x, \phi v_{1}, \ldots, \phi v_{k-1}\right) \\
& =\left(\phi^{*} \omega\right)_{x}\left(x, v_{1}, \ldots, v_{k-1}\right)=\left(\kappa \phi^{*} \omega\right)_{x}\left(v_{1}, \ldots, v_{k-1}\right) .
\end{aligned}
$$

For the case of an affine mapping $\phi x=\psi x+b$, with $\psi$ linear and $b \in \mathbb{R}^{n}$ a similar computation gives

$$
\begin{aligned}
\left(\phi^{*} \kappa \omega\right)_{x}\left(v_{1}, \ldots, v_{k-1}\right) & =(\kappa \omega)_{\phi x}\left(\psi v_{1}, \ldots, \psi v_{k-1}\right) \\
& =\omega_{\phi x}\left(\psi x+b, \psi x, \psi v_{1}, \ldots, \psi v_{k-1}\right) \\
& =\omega_{\phi x}\left(\psi x, \psi v_{1}, \ldots, \psi v_{k-1}\right)+\omega_{\phi x}\left(b, \psi v_{1}, \ldots, \psi v_{k-1}\right) \\
& =\left(\kappa \phi^{*} \omega\right)_{x}\left(v_{1}, \ldots, v_{k-1}\right)+\mu_{x}\left(v_{1}, \ldots, v_{k-1}\right)
\end{aligned}
$$

where $\mu_{x}\left(v_{1}, \ldots, v_{k-1}\right)=\omega_{\phi x}\left(b, \psi v_{1}, \ldots, \psi v_{k-1}\right)$, so $\mu \in \mathcal{P}_{r} \Lambda^{k-1}$. Thus

$$
\phi^{*} \kappa \omega-\kappa \phi^{*} \omega \in \mathcal{P}_{r} \Lambda^{k-1}, \quad \omega \in \mathcal{H}_{r} \Lambda^{k}
$$

The operator $\kappa$ maps the Koszul algebra $\mathcal{P} \Lambda$ to itself. There it is called the Koszul operator (Guillemin and Sternberg 1999, Chapter 3.1), and gives rise to the homogeneous Koszul complex (Loday 1992, Chapter 3.4.6),

$$
0 \rightarrow \mathcal{H}_{r-n} \Lambda^{n} \stackrel{\kappa}{\longrightarrow} \mathcal{H}_{r-n+1} \Lambda^{n-1} \stackrel{\kappa}{\longrightarrow} \cdots \stackrel{\kappa}{\longrightarrow} \mathcal{H}_{r} \Lambda^{0} \rightarrow 0 .
$$

We show below that this complex is exact for $r>0$. Adding over polynomial degrees, we obtain the Koszul complex (for any $r \geq 0$ ),

$$
0 \rightarrow \mathcal{P}_{r-n} \Lambda^{n} \stackrel{\kappa}{\longrightarrow} \mathcal{P}_{r-n+1} \Lambda^{n-1} \stackrel{\kappa}{\longrightarrow} \cdots \stackrel{\kappa}{\longrightarrow} \mathcal{P}_{r} \Lambda^{0} \rightarrow 0
$$

for which all the cohomology spaces vanish, except the right most, which is equal to $\mathbb{R}$.

To prove the exactness of the homogeneous polynomial de Rham and Koszul complexes, we establish a key connection between the exterior derivative and the Koszul differential. In the language of homological algebra, this says that the Koszul operator is a contracting homotopy for the homogeneous polynomial de Rham complex.

\section{Theorem 3.1.}

$$
(\mathrm{d} \kappa+\kappa \mathrm{d}) \omega=(r+k) \omega, \quad \omega \in \mathcal{H}_{r} \Lambda^{k} .
$$

Proof. It suffices to prove the result for $\omega=f \mathrm{~d} x_{\sigma(1)} \wedge \cdots \wedge \mathrm{d} x_{\sigma(k)}$ where $1 \leq \sigma(1)<\cdots<\sigma(k) \leq n$, and $f \in \mathcal{H}_{r}$. To simplify notation, we may as well assume that $\sigma(i)=i$, so $\omega=f \mathrm{~d} x_{1} \wedge \cdots \wedge \mathrm{d} x_{k}$. Now

$$
\begin{aligned}
\kappa \mathrm{d} \omega & =\kappa\left(\sum_{i=1}^{n} \frac{\partial f}{\partial x_{i}} \mathrm{~d} x_{i} \wedge \mathrm{d} x_{1} \wedge \cdots \wedge \mathrm{d} x_{k}\right) \\
& =\sum_{i=1}^{n} \kappa\left(\frac{\partial f}{\partial x_{i}} \mathrm{~d} x_{i} \wedge \mathrm{d} x_{1} \wedge \cdots \wedge \mathrm{d} x_{k}\right)
\end{aligned}
$$


Finite ELEMENT EXTERIOR CALCULUS

$$
\begin{aligned}
& =\sum_{i=1}^{n} \frac{\partial f}{\partial x_{i}}\left[x_{i} \mathrm{~d} x_{1} \wedge \cdots \wedge \mathrm{d} x_{k}\right. \\
& \left.\quad+\sum_{j=1}^{k}(-1)^{j} x_{j} \mathrm{~d} x_{i} \wedge \mathrm{d} x_{1} \wedge \cdots \wedge \widehat{\mathrm{d}}_{j} \wedge \cdots \wedge \mathrm{d} x_{k}\right] \\
& =r \omega+\sum_{i=1}^{n} \sum_{j=1}^{k}(-1)^{j} \frac{\partial f}{\partial x_{i}} x_{j} \mathrm{~d} x_{i} \wedge \mathrm{d} x_{1} \wedge \cdots \wedge \widehat{\mathrm{d}}_{j} \wedge \cdots \wedge \mathrm{d} x_{k} .
\end{aligned}
$$

In the last step we have used Euler's identity $\sum_{i} x_{i} \partial f / \partial x_{i}=r f$ for $f \in \mathcal{H}_{r}$.

On the other hand,

$$
\begin{aligned}
\mathrm{d} \kappa \omega & =\mathrm{d}\left[\sum_{j=1}^{k}(-1)^{j-1} f x_{j} \mathrm{~d} x_{1} \wedge \cdots \wedge \widehat{\mathrm{d}}_{j} \wedge \cdots \wedge \mathrm{d} x_{k}\right] \\
& =\sum_{j=1}^{k} \sum_{i=1}^{n}(-1)^{j-1} \frac{\partial f x_{j}}{\partial x_{i}} \mathrm{~d} x_{i} \wedge \mathrm{d} x_{1} \wedge \cdots \wedge \widehat{\mathrm{d}}_{j} \wedge \cdots \wedge \mathrm{d} x_{k} \\
& =\sum_{j=1}^{k} \sum_{i=1}^{n}(-1)^{j-1}\left(f \delta_{i j}+\frac{\partial f}{\partial x_{i}} x_{j}\right) \mathrm{d} x_{i} \wedge \mathrm{d} x_{1} \wedge \cdots \wedge \widehat{\mathrm{d}}_{j} \wedge \cdots \wedge \mathrm{d} x_{k} \\
& =k \omega+\sum_{j=1}^{k} \sum_{i=1}^{n}(-1)^{j-1} \frac{\partial f}{\partial x_{i}} x_{j} \mathrm{~d} x_{i} \wedge \mathrm{d} x_{1} \wedge \cdots \wedge \widehat{\mathrm{d}}_{j} \wedge \cdots \wedge \mathrm{d} x_{k} .
\end{aligned}
$$

Adding these two expressions gives the desired result.

Remark. An alternative proof of the theorem is based on the homotopy formula of differential geometry (see Lang (1995, Chapter V, Proposition 5.3) or Taylor (1996, Chapter 1, Proposition 13.1)), which states that for any vector field $v$ on a manifold

$$
\mathrm{d}(\omega\lrcorner v)+(\mathrm{d} \omega)\lrcorner v=\mathcal{L}_{v} \omega .
$$

Here $\mathcal{L}_{v} \omega$ denotes the Lie derivative of $\omega$ with respect to $v$, defined by

$$
\mathcal{L}_{v} \omega=\left.\frac{\mathrm{d}}{\mathrm{d} t}\left(\alpha_{t}^{*} \omega\right)\right|_{t=0}
$$

where $x \mapsto \alpha_{t}(x) \in \Omega$ is the flow defined for $(x, t)$ in a neighbourhood of $\Omega \times\{0\}$ in $\Omega \times \mathbb{R}$ by $\mathrm{d} \alpha_{t}(x) / \mathrm{d} t=v\left(\alpha_{t}(x)\right)$ and $\alpha_{0}(x)=x$. For the vector field $v=X$, it is easy to check that the flow is simply $\alpha_{t}(x)=e^{t} x$, so

$$
\left(\alpha_{t}^{*} \omega\right)_{x}\left(v_{1}, \ldots, v_{k}\right)=\omega_{e^{t} x}\left(e^{t} v_{1}, \ldots, e^{t} v_{k}\right)=e^{(r+k) t} \omega_{x}\left(v_{1}, \ldots, v_{k}\right),
$$

for $\omega \in \mathcal{H}_{r} \Lambda^{k}$. Differentiating with respect to $t$ and setting $t=0$, we obtain the desired result. 
As a simple consequence of Theorem 3.9, we prove the injectivity of $\mathrm{d}$ on the range of $\kappa$ and vice versa.

Theorem 3.2. If $\mathrm{d} \kappa \omega=0$ for some $\omega \in \mathcal{P} \Lambda$, then $\kappa \omega=0$. If $\kappa \mathrm{d} \omega=0$ for some $\omega \in \mathcal{P} \Lambda$, then $\mathrm{d} \omega=0$.

Proof. We may assume that $\omega \in \mathcal{H}_{r} \Lambda^{k}$ for some $r, k \geq 0$. If $r=k=0$, the result is trivial, so we may assume that $r+k>0$. Then

$$
(r+k) \kappa \omega=\kappa(\mathrm{d} \kappa+\kappa \mathrm{d}) \omega=0,
$$

if $\mathrm{d} \kappa \omega=0$, so $\kappa \omega=0$ in this case. Similarly,

$$
(r+k) \mathrm{d} \omega=\mathrm{d}(\mathrm{d} \kappa+\kappa \mathrm{d}) \omega=0,
$$

if $\kappa \mathrm{d} \omega=0$.

Another easy application of (3.9) is to establish the claimed cohomology of the Koszul complex and polynomial de Rham complex. Suppose that $\omega \in \mathcal{H}_{r} \Lambda^{k}$ for some $r, k \geq 0$ with $r+k>0$, and that $\kappa \omega=0$. From (3.9), we see that $\omega=\kappa \eta$ with $\eta=\mathrm{d} \omega /(r+k) \in \mathcal{H}_{r-1} \Lambda^{k+1}$. This establishes the exactness of the homogeneous Koszul complex (3.8) (except when $r=0$ and the sequence reduces to $0 \rightarrow \mathbb{R} \rightarrow 0$ ). A similar argument establishes the exactness of (3.2).

Another immediate but important consequence of (3.9) is a direct sum decomposition of $\mathcal{H}_{r} \Lambda^{k}$ for $r, k \geq 0$ with $r+k>0$ :

$$
\mathcal{H}_{r} \Lambda^{k}=\kappa \mathcal{H}_{r-1} \Lambda^{k+1} \oplus \mathrm{d} \mathcal{H}_{r+1} \Lambda^{k-1}
$$

Indeed, if $\omega \in \mathcal{H}_{r} \Lambda^{k}$, then $\eta=\mathrm{d} \omega /(r+k) \in \mathcal{H}_{r-1} \Lambda^{k+1}$ and $\mu=\kappa \omega /(r+k) \in$ $\mathcal{H}_{r+1} \Lambda^{k-1}$ and $\omega=\kappa \eta+\mathrm{d} \mu$, so $\mathcal{H}_{r} \Lambda^{k}=\kappa \mathcal{H}_{r-1} \Lambda^{k+1}+\mathrm{d} \mathcal{H}_{r+1} \Lambda^{k-1}$. Also, if $\omega \in \kappa \mathcal{H}_{r-1} \Lambda^{k+1} \cap \mathrm{d} \mathcal{H}_{r+1} \Lambda^{k-1}$, the $\mathrm{d} \omega=\kappa \omega=0$ (since $\mathrm{d} \circ \mathrm{d}=\kappa \circ \kappa=0$ ), and so, by (3.9), $\omega=0$. This shows that the sum is direct. Since $\mathcal{P}_{r} \Lambda^{k}=$ $\bigoplus_{j=0}^{r} \mathcal{H}_{j} \Lambda^{k}$, we also have

$$
\mathcal{P}_{r} \Lambda^{k}=\kappa \mathcal{P}_{r-1} \Lambda^{k+1} \oplus \mathrm{d} \mathcal{P}_{r+1} \Lambda^{k-1} .
$$

We now use the exactness of the Koszul complex to compute the dimension of the summands in (3.10).

Theorem 3.3. Let $r \geq 0,1 \leq k \leq n$, for integers $r, k$, and $n$. Then

$$
\operatorname{dim} \kappa \mathcal{H}_{r} \Lambda^{k}\left(\mathbb{R}^{n}\right)=\operatorname{dim} \mathrm{d} \mathcal{H}_{r+1} \Lambda^{k-1}\left(\mathbb{R}^{n}\right)=\left(\begin{array}{c}
n+r \\
n-k
\end{array}\right)\left(\begin{array}{c}
r+k-1 \\
k-1
\end{array}\right) .
$$

Proof. Applying $\kappa$ to both sides of (3.10), we have

$$
\kappa \mathcal{H}_{r} \Lambda^{k}\left(\mathbb{R}^{n}\right)=\kappa \mathrm{d} \mathcal{H}_{r+1} \Lambda^{k-1}\left(\mathbb{R}^{n}\right) .
$$

But $\kappa$ is injective on the range of $\mathrm{d}$ by Theorem 3.2. Thus the first equality of (3.12) holds. 
We turn to the proof of the dimension formula

$$
\operatorname{dim} \kappa \mathcal{H}_{r} \Lambda^{k}\left(\mathbb{R}^{n}\right)=\left(\begin{array}{c}
n+r \\
n-k
\end{array}\right)\left(\begin{array}{c}
r+k-1 \\
k-1
\end{array}\right) .
$$

From the exactness properties of the Koszul complex, we know that the Koszul operator is injective on $\mathcal{H}_{0} \Lambda^{k}\left(\mathbb{R}^{n}\right)$ for all $k \geq 1$ and on $\mathcal{H}_{r} \Lambda^{n}\left(\mathbb{R}^{n}\right)$ for all $r \geq 0$, so the formula is trivially verified in these cases. Now the range of $\kappa$ acting on $\mathcal{H}_{r} \Lambda^{k}\left(\mathbb{R}^{n}\right)$ is equal to the dimension of $\mathcal{H}_{r} \Lambda^{k}\left(\mathbb{R}^{n}\right)$ minus the dimension of the null space of $\kappa$ on that space. By the exactness of the Koszul complex, the null space is $\kappa \mathcal{H}_{r-1} \Lambda^{k+1}\left(\mathbb{R}^{n}\right)$. By (3.1)

$$
\operatorname{dim} \mathcal{H}_{r} \Lambda^{k}\left(\mathbb{R}^{n}\right)=\operatorname{dim} \mathcal{P}_{r}\left(\mathbb{R}^{n}\right) \Lambda^{k}\left(\mathbb{R}^{n-1}\right)=\left(\begin{array}{c}
n+r-1 \\
n-1
\end{array}\right)\left(\begin{array}{l}
n \\
k
\end{array}\right) .
$$

Thus

$$
\operatorname{dim} \kappa \mathcal{H}_{r} \Lambda^{k}\left(\mathbb{R}^{n}\right)=\left(\begin{array}{c}
n+r-1 \\
n-1
\end{array}\right)\left(\begin{array}{l}
n \\
k
\end{array}\right)-\operatorname{dim} \kappa \mathcal{H}_{r-1} \Lambda^{k+1}\left(\mathbb{R}^{n}\right) .
$$

The dimension formula (3.13) follows from this equation and a backward induction on $k$, the case $k=n$ being known. Indeed suppose that (3.12) holds for all $r$ with $k$ replaced by $k+1$. Substituting this (with $r$ replaced by $r-1$ ) into (3.14) and using the binomial identity

$$
\left(\begin{array}{c}
n+r-1 \\
n-1
\end{array}\right)\left(\begin{array}{l}
n \\
k
\end{array}\right)-\left(\begin{array}{c}
n+r-1 \\
n-k-1
\end{array}\right)\left(\begin{array}{c}
r+k-1 \\
k
\end{array}\right)=\left(\begin{array}{c}
n+r \\
n-k
\end{array}\right)\left(\begin{array}{c}
r+k-1 \\
k-1
\end{array}\right)
$$

we obtain the result.

\subsection{The space $\mathcal{P}_{r}^{-} \Lambda^{k}$}

Let $r \geq 1$. Obviously, $\mathcal{P}_{r} \Lambda^{k}=\mathcal{P}_{r-1} \Lambda^{k}+\mathcal{H}_{r} \Lambda^{k}$. In view of (3.10) we may define a space of $k$-forms intermediate between $\mathcal{P}_{r-1} \Lambda^{k}$ and $\mathcal{P}_{r} \Lambda^{k}$ by

$$
\mathcal{P}_{r}^{-} \Lambda^{k}=\mathcal{P}_{r-1} \Lambda^{k}+\kappa \mathcal{H}_{r-1} \Lambda^{k+1}=\mathcal{P}_{r-1} \Lambda^{k}+\kappa \mathcal{P}_{r-1} \Lambda^{k+1} .
$$

Note that the first sum is direct, while the second need not be. An equivalent definition is

$$
\mathcal{P}_{r}^{-} \Lambda^{k}=\left\{\omega \in \mathcal{P}_{r} \Lambda^{k} \mid \kappa \omega \in \mathcal{P}_{r} \Lambda^{k-1}\right\} .
$$

Note that $\mathcal{P}_{r}^{-} \Lambda^{0}=\mathcal{P}_{r} \Lambda^{0}$ and $\mathcal{P}_{r}^{-} \Lambda^{n}=\mathcal{P}_{r-1} \Lambda^{n}$, but for $0<k<n, \mathcal{P}_{r}^{-} \Lambda^{k}$ is contained strictly between $\mathcal{P}_{r-1} \Lambda^{k}$ and $\mathcal{P}_{r} \Lambda^{k}$. For $r \leq 0$, we set $\mathcal{P}_{r}^{-} \Lambda^{k}=0$.

From (3.12), we have

$$
\begin{aligned}
\operatorname{dim} \mathcal{P}_{r}^{-} \Lambda^{k}\left(\mathbb{R}^{n}\right) & =\operatorname{dim} \mathcal{P}_{r-1} \Lambda^{k}+\operatorname{dim} \kappa \mathcal{H}_{r-1} \Lambda^{k+1} \\
& =\left(\begin{array}{c}
n+r-1 \\
n
\end{array}\right)\left(\begin{array}{l}
n \\
k
\end{array}\right)+\left(\begin{array}{l}
n+r-1 \\
n-k-1
\end{array}\right)\left(\begin{array}{c}
r+k-1 \\
k
\end{array}\right) \\
& =\left(\begin{array}{c}
r+k-1 \\
k
\end{array}\right)\left(\begin{array}{l}
n+r \\
n-k
\end{array}\right),
\end{aligned}
$$


where the last step is a simple identity.

Analogous to the obvious closure relation

$$
\mathcal{P}_{r} \Lambda^{k} \wedge \mathcal{P}_{s} \Lambda^{l} \subset \mathcal{P}_{r+s} \Lambda^{k+l},
$$

the $\mathcal{P}_{r}^{-} \Lambda^{k}$ spaces satisfy

$$
\mathcal{P}_{r}^{-} \Lambda^{k} \wedge \mathcal{P}_{s}^{-} \Lambda^{l} \subset \mathcal{P}_{r+s}^{-} \Lambda^{k+l},
$$

first proved in Christiansen (2005). To prove (3.16), it suffices to show that

$$
\kappa \mathcal{H}_{r-1} \Lambda^{k+1} \wedge \kappa \mathcal{H}_{s-1} \Lambda^{l+1} \subset \kappa \mathcal{H}_{r+s-1} \Lambda^{k+l-1} .
$$

By the exactness of the Koszul complex, it is enough to show that $\kappa(\kappa \omega \wedge$ $\kappa \mu)=0$. But this follows immediately from (3.5) and (3.6). Taking $l=0$ and noting that $\mathcal{P}_{s}^{-} \Lambda^{l}=\mathcal{P}_{s}$, we get

$$
p \in \mathcal{P}_{s}, \omega \in \mathcal{P}_{r}^{-} \Lambda^{k} \Longrightarrow p \omega \in \mathcal{P}_{r+s}^{-} \Lambda^{k} .
$$

We close by noting a simple consequence of Lemma 3.2.

Theorem 3.4. If $\omega \in \mathcal{P}_{r}^{-} \Lambda^{k}$ and $\mathrm{d} \omega=0$, then $\omega \in \mathcal{P}_{r-1}^{-} \Lambda^{k}$.

Proof. Write $\omega=\omega_{1}+\kappa \omega_{2}$ with $\omega_{1} \in \mathcal{P}_{r-1} \Lambda^{k}$ and $\omega_{2} \in \mathcal{P}_{r-1} \Lambda^{k+1}$. Then

$$
\mathrm{d} \omega=0 \Longrightarrow \mathrm{d} \kappa \omega_{2}=0 \Longrightarrow \kappa \omega_{2}=0 \Longrightarrow \omega \in \mathcal{P}_{r-1} \Lambda^{k} .
$$

\subsection{Invariant spaces of polynomial differential forms}

We have already noted in (3.4) that the spaces $\mathcal{P}_{r} \Lambda^{k}$ of polynomial differential forms are affine-invariant, i.e., mapped into themselves by the pullback of affine transformations of $\mathbb{R}^{n}$. This is a stronger property than linearinvariance (invariance under the pullback of linear transformations). For example, $\mathcal{H}_{r} \Lambda^{k}$ is linear-invariant, but not affine-invariant. Let us explain the significance of affine invariance for finite element spaces of differential forms. In the next section we define the space $\mathcal{P}_{r} \Lambda^{k}(T)$ for an $n$-simplex $T$ to be the space of restrictions of polynomials in $\mathcal{P}_{r} \Lambda^{k}$ to the simplex. In the following section we define the finite element space $\mathcal{P}_{r} \Lambda^{k}\left(\mathcal{T}_{h}\right)$ for a simplicial complex $\mathcal{T}_{h}$ consisting of piecewise polynomial differential forms which restrict to $\mathcal{P}_{r} \Lambda^{k}(T)$ on each $T \in \mathcal{T}_{h}$. Another possible construction would be to select a single reference simplex $\hat{T}$, and define $\mathcal{P}_{r} \Lambda^{k}(\hat{T})$, and then to define $\mathcal{P}_{r} \Lambda^{k}(T)=\Phi^{*}\left(\mathcal{P}_{r} \Lambda^{k}(\hat{T})\right)$ for any other simplex $T$, where $\Phi: \hat{T} \rightarrow T$ is an affine isomorphism. Affine invariance shows that these two definitions of $\mathcal{P}_{r} \Lambda^{k}(T)$ are the same (and so the space does not depend on the choice of affine isomorphism $\Phi$ of $\hat{T}$ on $T$ ).

It is relatively easy matter to see that the only finite-dimensional affineinvariant spaces of polynomial 0-forms (i.e., ordinary polynomial functions) are the spaces $\mathcal{P}_{r} \Lambda^{0}$ for $r=0,1,2, \ldots$ (and this will follow from the techniques below). Similarly, the only finite-dimensional affine-invariant spaces 
of polynomial $n$-forms are the spaces $\mathcal{P}_{r} \Lambda^{n}$. However, for $0<k<n$, there are other affine-invariant spaces of polynomial $k$-forms. Specifically, the spaces $\mathcal{P}_{r}^{-} \Lambda^{k}, r=1,2, \ldots$, are affine-invariant. In this subsection, we shall determine all the affine-invariant subspaces of polynomial $k$-forms.

First we note that the decomposition of $\mathcal{H}_{r} \Lambda^{k}$ given in (3.10) is a decomposition into subspaces which are linear-invariant (but not affine-invariant). Indeed, if $\phi: \mathbb{R}^{n} \rightarrow \mathbb{R}^{n}$ is linear, then from the relations $\phi^{*} \mathrm{~d}=\mathrm{d} \phi^{*}$ (which holds for any transformation $\phi$ ), and $\phi^{*} \kappa=\kappa \phi^{*}$ (which holds for $\phi$ linear), and the invariance under linear transformations of the spaces of the homogeneous forms, we have

$$
\begin{aligned}
\phi^{*} \mathrm{~d} \mathcal{H}_{r+1} \Lambda^{k-1} & =\mathrm{d} \phi^{*} \mathcal{H}_{r+1} \Lambda^{k-1} \subset \mathrm{d} \mathcal{H}_{r+1} \Lambda^{k-1}, \\
\phi^{*} \kappa \mathcal{H}_{r-1} \Lambda^{k+1} & =\kappa \phi^{*} \mathcal{H}_{r-1} \Lambda^{k+1} \subset \kappa \mathcal{H}_{r-1} \Lambda^{k+1}
\end{aligned}
$$

This establishes the invariance of the summands.

The same argument shows that the space $\mathrm{d} \mathcal{P}_{r+1} \Lambda^{k-1}$ is invariant under affine transformations:

$$
\phi^{*} \mathrm{~d} \mathcal{P}_{r+1} \Lambda^{k-1}=\mathrm{d} \phi^{*} \mathcal{P}_{r+1} \Lambda^{k-1} \subset \mathrm{d} \mathcal{P}_{r+1} \Lambda^{k-1} .
$$

For the range of $\kappa$, we can only get a weaker result using (3.7), namely

$$
\phi^{*} \kappa \mathcal{P}_{r-1} \Lambda^{k+1} \subset \kappa \phi^{*} \mathcal{P}_{r-1} \Lambda^{k+1}+\mathcal{P}_{r-1} \Lambda^{k} \subset \kappa \mathcal{P}_{r-1} \Lambda^{k+1}+\mathcal{P}_{r-1} \Lambda^{k} .
$$

We now combine these results to find several affine-invariant subspaces of $\mathcal{P}_{r} \Lambda^{k}$. Below we shall show that these are the only such subspaces.

Theorem 3.5. Let $0<k<n, r \geq 0,-1 \leq s \leq r$, for integers $k, n, r$, and $s$. Then the space

$$
X(r, s, k, n):=\mathrm{d} \mathcal{P}_{r+1} \Lambda^{k-1}\left(\mathbb{R}^{n}\right)+\kappa \mathcal{P}_{s} \Lambda^{k+1}\left(\mathbb{R}^{n}\right)
$$

is an affine-invariant subspace of $\mathcal{P} \Lambda^{k}\left(\mathbb{R}^{n}\right)$. Furthermore:

- $($ case $s=r) X(r, r, k, n)=\mathcal{P}_{r+1}^{-} \Lambda^{k}\left(\mathbb{R}^{n}\right)$;

- $($ case $s=r-1) X(r, r-1, k, n)=\mathcal{P}_{r} \Lambda^{k}\left(\mathbb{R}^{n}\right)$;

- (case $s<r-1)$ if $-1 \leq s<r-1$, then

$$
\begin{aligned}
X(r, s, k, n) & =\mathcal{P}_{s+1} \Lambda^{k}\left(\mathbb{R}^{n}\right)+\mathrm{d} \mathcal{P}_{r+1} \Lambda^{k-1}\left(\mathbb{R}^{n}\right) \\
& =\left\{\omega \in \mathcal{P}_{r} \Lambda^{k}\left(\mathbb{R}^{n}\right) \mid \mathrm{d} \omega \in \mathcal{P}_{s} \Lambda^{k}\left(\mathbb{R}^{n}\right)\right\} .
\end{aligned}
$$

This space is contained strictly between $\mathcal{P}_{s+1} \Lambda^{k}$ and $\mathcal{P}_{r} \Lambda^{k}$, but does not contain $\mathcal{P}_{s+2} \Lambda^{k}$.

Proof. From (3.18), (3.19), (3.11), and the fact that $s \leq r$, we have

$$
\begin{aligned}
\phi^{*} X(r, s, k, n) & \subset \mathrm{d} \mathcal{P}_{r+1} \Lambda^{k-1}+\kappa \mathcal{P}_{s} \Lambda^{k+1}+\mathcal{P}_{s} \Lambda^{k} \\
& =\mathrm{d} \mathcal{P}_{r+1} \Lambda^{k-1}+\kappa \mathcal{P}_{s} \Lambda^{k+1}+\mathrm{d} \mathcal{P}_{s+1} \Lambda^{k-1}+\kappa \mathcal{P}_{s-1} \Lambda^{k-1} \\
& =\mathrm{d} \mathcal{P}_{r+1} \Lambda^{k-1}+\kappa \mathcal{P}_{s} \Lambda^{k+1}=X(r, s, k, n),
\end{aligned}
$$


which is the claimed invariance. The bulleted points are then simple observations.

Thus, for $0<k<n$, there are three distinct types of finite-dimensional affine-invariant spaces of polynomial differential $k$-forms (and, as we shall soon show, that these are the only ones):

- the spaces $\mathcal{P}_{r} \Lambda^{k}$ of all polynomial $k$-forms up to a given degree, $r$;

- the reduced spaces $\mathcal{P}_{r}^{-} \Lambda^{k}$; and

- the spaces consisting of all $\omega \in \mathcal{P}_{r} \Lambda^{k}$ for which the exterior derivative $\mathrm{d} \omega$ is constrained to belong to $\mathcal{P}_{s} \Lambda^{k}$ for some $-1 \leq s<r-1$ (with $s=-1$ corresponding to the constraint $\mathrm{d} \omega=0)$.

In this paper we will investigate spaces of piecewise polynomial differential forms for which the pieces belong to one of the spaces $\mathcal{P}_{r} \Lambda^{k}$ or $\mathcal{P}_{r}^{-} \Lambda^{k}$, i.e., to spaces of the first or second kind listed. The third class of spaces will not be considered. Up until now, these spaces have not been widely used as mixed finite element spaces, and it is not clear that there is a motivation to do so. However, a vector-valued analogue of these spaces played a major role in the development of stable mixed finite elements for elasticity in Arnold and Winther (2002).

In the remainder of this subsection, we show that the spaces $X(r, s, k, n)$ given in Theorem 3.5 are the only finite-dimensional affine-invariant subspaces of $\mathcal{P} \Lambda^{k}$. This result will not be needed later, and so the reader uninterested in the proof may safely skip ahead to Section 3.5.

Theorem 3.6. Let $0<k<n$, and suppose that $X \subset \mathcal{P} \Lambda^{k}$ is a nonzero finite-dimensional subspace satisfying $\phi^{*} X \subset X$ for all affine maps $\phi: \mathbb{R}^{n} \rightarrow$ $\mathbb{R}^{n}$. Then $X=X(r, s, k, n)$ for some integers $r, s$, with $r \geq 0,-1 \leq s \leq r$.

The proof will be based on the representation theory of the general linear group, for which we will first summarize the main results needed. These results may be gleaned from Fulton and Harris (1991), especially Section 6.1. Via the pullback, the group $\operatorname{GL}\left(\mathbb{R}^{n}\right)$ acts on $\mathcal{P} \Lambda^{k}$ and on its subspace $\mathcal{H}_{r} \Lambda^{k}$. From equation (6.9) of Fulton and Harris (1991) in the case $\lambda=(r)$ and $m=k$, and the accompanying discussion, we find that $\mathcal{H}_{r} \Lambda^{k}$ (which is $\mathrm{Sym}^{r} V \otimes \bigwedge^{k} V$ in the notation of Fulton and Harris (1991)) has precisely two nonzero proper invariant subspaces under this action. Since we have already established that $\mathrm{d} \mathcal{H}_{r+1} \Lambda^{k-1}$ and $\kappa \mathcal{H}_{r-1} \Lambda^{k+1}$ are such subspaces, the decomposition (3.10) is the decomposition of $\mathcal{H}_{r} \Lambda^{k}$ into irreducible linear-invariant subspaces. Moreover, all the nonzero spaces $\mathrm{d} \mathcal{H}_{r+1} \Lambda^{k-1}, \kappa \mathcal{H}_{r-1} \Lambda^{k+1}$ (for varying $r$ and $k$ ) are inequivalent as representations, because, as explained in Fulton and Harris (1991), they are the images of projections associated with different partitions (or different Young diagrams). This means that there does not exist a linear isomorphism between any two of them which 
commutes with the pullback action. Consequently, we may write down the decomposition of $\mathcal{P} \Lambda^{k}=\bigoplus_{r=0} \mathcal{H}_{r} \Lambda^{k}$ into irreducible linear-invariant subspaces,

$$
\mathcal{P} \Lambda^{k}=\bigoplus_{j=1}^{\infty} \mathrm{d} \mathcal{H}_{j} \Lambda^{k-1} \oplus \bigoplus_{i=0}^{\infty} \kappa \mathcal{H}_{i} \Lambda^{k+1}
$$

and from this decomposition we can read off all the finite-dimensional linearinvariant subspaces of $\mathcal{P}_{r} \Lambda^{k}$ : they are just the sums of some finite number of the summands appearing in (3.20).

The next step is to determine which of the linear-invariant subspaces is actually affine-invariant. We shall do this by considering the effect of the pullback by the translation operation. First we introduce some notation. Let

$$
\pi_{r}^{\mathrm{d}}: \mathcal{P} \Lambda^{k} \rightarrow \mathrm{d} \mathcal{H}_{r+1} \Lambda^{k-1}, \quad \pi_{s}^{\kappa}: \mathcal{P} \Lambda^{k} \rightarrow \kappa \mathcal{H}_{s-1} \Lambda^{k+1}
$$

denote the projections determined by the decomposition (3.20). Denote by $\tau: \mathbb{R}^{n} \rightarrow \mathbb{R}^{n}$ the unit translation in the $x_{1}$ direction:

$$
\tau(x)=\left(x_{1}+1, x_{2}, \ldots, x_{n}\right) .
$$

The proof of Theorem 3.6 will follow easily from the next lemma.

Lemma 3.7. Let $0<k<n$.

(1) For any $r \geq 1$, there exists $\omega \in \mathrm{d} \mathcal{H}_{r+1} \Lambda^{k-1}$ such that $\pi_{r-1}^{\mathrm{d}}\left(\tau^{*} \omega\right) \neq 0$.

(2) For any $s \geq 2$, there exists $\omega \in \kappa \mathcal{H}_{s-1} \Lambda^{k+1}$ such that $\pi_{s-1}^{\kappa}\left(\tau^{*} \omega\right) \neq 0$.

(3) For any $s \geq 1$, there exists $\omega \in \kappa \mathcal{H}_{s-1} \Lambda^{k+1}$ such that $\pi_{s-1}^{\mathrm{d}}\left(\tau^{*} \omega\right) \neq 0$.

Proof. For the proof we will exhibit such forms $\omega$ explicitly, and verify the result by direct computation.

(1) Let

$$
\omega=(r+1)^{-1} \mathrm{~d}\left(x_{1}^{r+1} \mathrm{~d} x_{2} \wedge \cdots \wedge \mathrm{d} x_{k}\right)=x_{1}^{r} \mathrm{~d} x_{1} \wedge \cdots \wedge \mathrm{d} x_{k} \in \mathrm{d} \mathcal{H}_{r+1} \Lambda^{k-1} .
$$

Then

$$
\begin{aligned}
\tau^{*} \omega & =\left(x_{1}+1\right)^{r} \mathrm{~d} x_{1} \wedge \cdots \wedge \mathrm{d} x_{k} \\
& =x_{1}^{r} \mathrm{~d} x_{1} \wedge \cdots \wedge \mathrm{d} x_{k}+r x_{1}^{r-1} \mathrm{~d} x_{1} \wedge \cdots \wedge \mathrm{d} x_{k}+\cdots,
\end{aligned}
$$

where we have expanded by polynomial degree. The term of degree $r-1$ is

$$
r x_{1}^{r-1} \mathrm{~d} x_{1} \wedge \cdots \wedge \mathrm{d} x_{k}=d\left(x_{1}^{r} \mathrm{~d} x_{2} \wedge \cdots \wedge \mathrm{d} x_{k}\right) \in \mathrm{d} \mathcal{H}_{r} \Lambda^{k-1} .
$$

Therefore $\pi_{r-1}^{\mathrm{d}}\left(\tau^{*} \omega\right)=r x_{1}^{r-1} \mathrm{~d} x_{1} \wedge \cdots \wedge \mathrm{d} x_{k} \neq 0$. 
(2) Let

$$
\begin{aligned}
\omega & =\kappa\left(x_{1}^{s-1} \mathrm{~d} x_{1} \wedge \cdots \wedge \mathrm{d} x_{k+1}\right) \\
& =x_{1}^{s-1} \sum_{j=1}^{k+1}(-1)^{j+1} x_{j} \mathrm{~d} x_{1} \wedge \cdots \wedge \widehat{\mathrm{d}}_{j} \wedge \cdots \wedge \mathrm{d} x_{k+1} \in \kappa \mathcal{H}_{s-1} \Lambda^{k+1} .
\end{aligned}
$$

Then

$$
\begin{aligned}
\tau^{*} \omega=\left(x_{1}+1\right)^{s} \mathrm{~d} x_{2} & \wedge \cdots \wedge \mathrm{d} x_{k+1} \\
& +\left(x_{1}+1\right)^{s-1} \sum_{j=2}^{k+1}(-1)^{j+1} x_{j} \mathrm{~d} x_{1} \wedge \cdots \wedge \widehat{\mathrm{d} x}_{j} \wedge \cdots \wedge \mathrm{d} x_{k+1} .
\end{aligned}
$$

Letting $\mu=\pi_{s-1}\left(\tau^{*} \omega\right)$, where $\pi_{s-1}: \mathcal{P} \Lambda^{k} \rightarrow \mathcal{H}_{s-1} \Lambda^{k}$ is the projection onto homogeneous polynomial forms of degree $s-1$, we get

$$
\begin{aligned}
& \mu=s x_{1}^{s-1} \mathrm{~d} x_{2} \wedge \cdots \wedge \mathrm{d} x_{k+1} \\
&+(s-1) x_{1}^{s-2} \sum_{j=2}^{k+1}(-1)^{j+1} x_{j} \mathrm{~d} x_{1} \wedge \cdots \wedge \widehat{\mathrm{d}}_{j} \wedge \cdots \wedge \mathrm{d} x_{k+1} .
\end{aligned}
$$

Then

$$
\begin{aligned}
\mathrm{d} \mu= & s(s-1) x_{1}^{s-1} \mathrm{~d} x_{1} \wedge \cdots \wedge \mathrm{d} x_{k+1} \\
& \quad+(s-1) x_{1}^{s-2} \sum_{j=2}^{k+1}(-1)^{j+1} \mathrm{~d} x_{j} \wedge \cdots \wedge \widehat{\mathrm{d}}_{j} \wedge \cdots \wedge \mathrm{d} x_{k+1} \\
= & \left(s^{2}-1\right) x_{1}^{s-1} \mathrm{~d} x_{1} \wedge \cdots \wedge \mathrm{d} x_{k+1} \neq 0 .
\end{aligned}
$$

Thus $\mu \in \mathcal{H}_{s-1} \Lambda^{k}$, but $\mu \notin \mathrm{d} \mathcal{H}_{s} \Lambda^{k+1}$. Therefore $\pi_{s-1}^{\mathrm{d}}\left(\tau^{*} \omega\right)=\pi_{s-1}^{\mathrm{d}} \mu \neq 0$.

(3) Let

$\omega=\kappa\left(x_{n}^{s} \mathrm{~d} x_{1} \wedge \cdots \wedge \mathrm{d} x_{k+1}\right)=x_{n}^{s} \sum_{j=1}^{k+1}(-1)^{j+1} x_{j} \mathrm{~d} x_{1} \wedge \cdots \wedge \widehat{\mathrm{d}}_{j} \wedge \cdots \wedge \mathrm{d} x_{k+1}$.

Then $\tau^{*} \omega=\omega+\mu$ where $\mu=x_{n}^{s} \mathrm{~d} x_{2} \wedge \cdots \wedge \mathrm{d} x_{k+1}$. Now

$$
\kappa \mu=x_{n}^{s} \sum_{j=2}^{k+1}(-1)^{j} x_{j} \mathrm{~d} x_{2} \wedge \cdots \wedge \widehat{\mathrm{d}}_{j} \wedge \cdots \wedge \mathrm{d} x_{k+1} \neq 0 .
$$

Thus $\mu \in \mathcal{H}_{s} \Lambda^{k-1}$ but $\mu \notin \kappa \mathcal{H}_{s-1} \Lambda^{k}$, so $\pi_{s+1}^{\mathrm{d}} \tau^{*} \omega=\pi_{s+1}^{\mathrm{d}} \tau^{*} \mu \neq 0$.

Proof of Theorem 3.6. Finally, we complete the proof of Theorem 3.6. If $X$ is an affine-invariant subspace of $\mathcal{P} \Lambda^{k}$, it is a fortiori linear-invariant, and so a sum of finitely many summands from (3.20). Also $\tau^{*} X \subset X$. Suppose that 
$\mathrm{d} \mathcal{H}_{r+1} \Lambda^{k-1}$ is the highest degree summand in the range of $\mathrm{d}$. It follows from the first statement of the lemma that $\mathrm{d} \mathcal{H}_{r} \Lambda^{k-1}$ must then be a summand as well, and so, by induction, $\mathrm{d} \mathcal{P}_{r+1} \Lambda^{k-1} \subset X$. Similarly, if $\kappa \mathcal{H}_{s} \Lambda^{k+1}$ is the highest degree summand in the range of $\kappa$, the second statement of the lemma ensures that $\kappa \mathcal{P}_{s} \Lambda^{k+1} \subset X$. Finally, the third statement of the lemma ensures that $r \geq s$. Thus $X=\mathrm{d} \mathcal{P}_{r+1} \Lambda^{k-1}+\kappa \mathcal{P}_{s} \Lambda^{k+1}=X(r, s, k, n)$. This completes the proof.

\subsection{Exact sequences of polynomial differential forms}

We have seen the polynomial de Rham complex (3.3) is a subcomplex of the de Rham complex on $\mathbb{R}^{n}$ for which cohomology vanishes except for the constants at the lowest order. In other words, the sequence

$$
\mathbb{R} \hookrightarrow \mathcal{P}_{r} \Lambda^{0} \stackrel{\mathrm{d}}{\longrightarrow} \mathcal{P}_{r-1} \Lambda^{1} \stackrel{\mathrm{d}}{\longrightarrow} \cdots \stackrel{\mathrm{d}}{\longrightarrow} \mathcal{P}_{r-n} \Lambda^{n} \rightarrow 0
$$

is exact for any $r \geq 0$ (some of the spaces vanish if $r<n$ ). Such a complex, namely one which begins with the inclusion of $\mathbb{R}$, has vanishing cohomology, and terminates at 0 , is called a resolution of $\mathbb{R}$.

As we shall soon verify, the complex

$$
\mathbb{R} \hookrightarrow \mathcal{P}_{r}^{-} \Lambda^{0} \stackrel{\mathrm{d}}{\longrightarrow} \mathcal{P}_{r}^{-} \Lambda^{1} \stackrel{\mathrm{d}}{\longrightarrow} \cdots \stackrel{\mathrm{d}}{\longrightarrow} \mathcal{P}_{r}^{-} \Lambda^{n} \rightarrow 0
$$

is another resolution of $\mathbb{R}$, for any $r>0$. Note that in this complex, involving the $\mathcal{P}_{r}^{-} \Lambda^{k}$ spaces, the polynomial degree $r$ is held fixed, while in (3.21), the polynomial degree decreases as the form order increases. Recall that the 0th order spaces in these complexes, $\mathcal{P}_{r} \Lambda^{0}$ and $\mathcal{P}_{r}^{-} \Lambda^{0}$, coincide. In fact, the complex (3.21) is a subcomplex of (3.22), and these two are the extreme cases of a set of $2^{n-1}$ different resolutions of $\mathbb{R}$, each a subcomplex of the next, and all of which have the space $\mathcal{P}_{r} \Lambda^{0}$ in the 0 th order.

To prove all this, we first prove a simple lemma.

\section{Lemma 3.8.}

(1) For $r \geq 1, \mathrm{~d} \mathcal{P}_{r}^{-} \Lambda^{k} \subset \mathrm{d} \mathcal{P}_{r} \Lambda^{k} \subset \mathcal{P}_{r-1} \Lambda^{k+1} \subset \mathcal{P}_{r}^{-} \Lambda^{k+1}$.

(2) The following four restrictions of $\mathrm{d}$ each have the same kernel:

$$
\begin{array}{ll}
\mathrm{d}: \mathcal{P}_{r} \Lambda^{k} \rightarrow \mathcal{P}_{r-1} \Lambda^{k+1}, & \mathrm{~d}: \mathcal{P}_{r} \Lambda^{k} \rightarrow \mathcal{P}_{r}^{-} \Lambda^{k+1}, \\
\mathrm{~d}: \mathcal{P}_{r+1}^{-} \Lambda^{k} \rightarrow \mathcal{P}_{r} \Lambda^{k+1}, & \mathrm{~d}: \mathcal{P}_{r+1}^{-} \Lambda^{k} \rightarrow \mathcal{P}_{r+1}^{-} \Lambda^{k+1}
\end{array}
$$

(3) The following four restrictions of $d$ each have the same image:

$$
\begin{array}{ll}
\mathrm{d}: \mathcal{P}_{r} \Lambda^{k} \rightarrow \mathcal{P}_{r-1} \Lambda^{k+1}, & \mathrm{~d}: \mathcal{P}_{r} \Lambda^{k} \rightarrow \mathcal{P}_{r}^{-} \Lambda^{k+1} \\
\mathrm{~d}: \mathcal{P}_{r}^{-} \Lambda^{k} \rightarrow \mathcal{P}_{r-1} \Lambda^{k+1}, & \mathrm{~d}: \mathcal{P}_{r}^{-} \Lambda^{k} \rightarrow \mathcal{P}_{r}^{-} \Lambda^{k+1}
\end{array}
$$

Proof. The first statement is clear. To prove the second, we need to show that if $\omega \in \mathcal{P}_{r+1}^{-} \Lambda^{k}$ with $\mathrm{d} \omega=0$, then $\omega \in \mathcal{P}_{r} \Lambda^{k}$. We write $\omega=\omega_{1}+\kappa \omega_{2}$, with $\omega_{1} \in \mathcal{P}_{r} \Lambda^{k}$ and $\omega_{2} \in \mathcal{H}_{r} \Lambda^{k+1}$. Then $\mathrm{d} \omega=0$ if and only if $\mathrm{d} \omega_{1}=0$ 
and $\mathrm{d} \kappa \omega_{2}=0$. But $\mathrm{d}$ is injective on the range of $\kappa$, so $\kappa \omega_{2}=0$, so $\omega=\omega_{1} \in \mathcal{P}_{r} \Lambda^{k}$. Finally, to prove the third statement, it suffices to note that $\mathcal{P}_{r} \Lambda^{k}=\mathcal{P}_{r}^{-} \Lambda^{k}+\mathrm{d} \mathcal{P}_{r+1} \Lambda^{k-1}$, so $\mathrm{d} \mathcal{P}_{r} \Lambda^{k}=\mathrm{d} \mathcal{P}_{r}^{-} \Lambda^{k}$.

We now exhibit $2^{n-1}$ resolutions of $\mathbb{R}$, subcomplexes of the de Rham complex, beginning $\mathbb{R} \hookrightarrow \mathcal{P}_{r} \Lambda^{0}$. In view of the lemma, we may continue the complex with the map $\mathrm{d}: \mathcal{P}_{r} \Lambda^{0} \rightarrow \mathcal{P}_{r-1} \Lambda^{1}$ or $\mathrm{d}: \mathcal{P}_{r} \Lambda^{0} \rightarrow \mathcal{P}_{r}^{-} \Lambda^{1}$. The former is a subcomplex of the latter. With either choice, the cohomology vanishes at the first position. (In the former case we use the cohomology of (3.21), and in the latter we get the same result thanks to the lemma.)

Next, if we made the first choice, we can continue the complex with either $\mathrm{d}: \mathcal{P}_{r-1} \Lambda^{1} \rightarrow \mathcal{P}_{r-2} \Lambda^{2}$ or $\mathrm{d}: \mathcal{P}_{r-1} \Lambda^{1} \rightarrow \mathcal{P}_{r-1}^{-} \Lambda^{2}$. Or, if we made the second choice, we can continue with either $\mathrm{d}: \mathcal{P}_{r}^{-} \Lambda^{1} \rightarrow \mathcal{P}_{r-1} \Lambda^{2}$ or $\mathrm{d}: \mathcal{P}_{r}^{-} \Lambda^{1} \rightarrow \mathcal{P}_{r}^{-} \Lambda^{2}$. In any case, we may use the lemma and the exactness of (3.21) to see that the second cohomology space vanishes. Continuing in this way at each order, $k=1, \ldots, n-1$, we have two choices for the space of $k$-forms (but only one choice for $k=n$, since $\mathcal{P}_{r-1} \Lambda^{n}$ coincides with $\mathcal{P}_{r}^{-} \Lambda^{n}$ ), and so we obtain $2^{n-1}$ complexes. These form a totally ordered set with respect to subcomplexes. For $r \geq n$ these are all distinct (but for small $r$ some coincide because the later spaces vanish).

In the case $n=3$, the four complexes so obtained are:

$$
\begin{aligned}
& \mathbb{R} \hookrightarrow \mathcal{P}_{r} \Lambda^{0} \stackrel{\mathrm{d}}{\longrightarrow} \mathcal{P}_{r-1} \Lambda^{1} \stackrel{\mathrm{d}}{\longrightarrow} \mathcal{P}_{r-2} \Lambda^{2} \stackrel{\mathrm{d}}{\longrightarrow} \mathcal{P}_{r-3} \Lambda^{3} \rightarrow 0, \\
& \mathbb{R} \hookrightarrow \mathcal{P}_{r} \Lambda^{0} \stackrel{\mathrm{d}}{\longrightarrow} \mathcal{P}_{r-1} \Lambda^{1} \stackrel{\mathrm{d}}{\longrightarrow} \mathcal{P}_{r-1}^{-} \Lambda^{2} \stackrel{\mathrm{d}}{\longrightarrow} \mathcal{P}_{r-2} \Lambda^{3} \rightarrow 0, \\
& \mathbb{R} \hookrightarrow \mathcal{P}_{r} \Lambda^{0} \stackrel{\mathrm{d}}{\longrightarrow} \mathcal{P}_{r}^{-} \Lambda^{1} \stackrel{\mathrm{d}}{\longrightarrow} \mathcal{P}_{r-1} \Lambda^{2} \stackrel{\mathrm{d}}{\longrightarrow} \mathcal{P}_{r-2} \Lambda^{3} \rightarrow 0, \\
& \mathbb{R} \hookrightarrow \mathcal{P}_{r} \Lambda^{0} \stackrel{\mathrm{d}}{\longrightarrow} \mathcal{P}_{r}^{-} \Lambda^{1} \stackrel{\mathrm{d}}{\longrightarrow} \mathcal{P}_{r}^{-} \Lambda^{2} \stackrel{\mathrm{d}}{\longrightarrow} \mathcal{P}_{r-1} \Lambda^{3} \rightarrow 0 .
\end{aligned}
$$

\subsection{Change of origin}

We defined the Koszul differential as $\kappa=\lrcorner X$, where $X(x)$ is the translation to $x$ of the vector pointing from the origin in $\mathbb{R}^{n}$ to $x$. The choice of the origin as a base point is arbitrary - any point in $\mathbb{R}^{n}$ could be used. That is, if $y \in \mathbb{R}^{n}$, we can define a vector field $X_{y}$ by assigning to each point $x$ the translation to $x$ of the vector pointing from $y$ to $x$, and then define a Koszul operator $\left.\kappa_{y}=\right\lrcorner X_{y}$. It is easy to check that for $\omega \in \mathcal{P}_{r-1} \Lambda^{k+1}$ and any two points $y, y^{\prime} \in \mathbb{R}^{n}$, the difference $\kappa_{y} \omega-\kappa_{y^{\prime}} \omega \in \mathcal{P}_{r-1} \Lambda^{k}$. Hence the space

$$
\mathcal{P}_{r}^{-} \Lambda^{k}=\mathcal{P}_{r-1} \Lambda^{k}+\kappa_{y} \mathcal{P}_{r-1} \Lambda^{k+1}
$$

does not depend on the particular choice of the point $y$. This observation is important, because it allows us to define $\mathcal{P}_{r}^{-} \Lambda^{k}(V)$ for any affine subspace 
$V$ of $\mathbb{R}^{n}$. We simply set

$$
\mathcal{P}_{r}^{-} \Lambda^{k}(V)=\mathcal{P}_{r-1} \Lambda^{k}(V)+\kappa_{y} \mathcal{P}_{r-1} \Lambda^{k+1}(V),
$$

where $y$ is any point of $V$. Note that if $\omega \in \mathcal{P}_{r}^{-} \Lambda^{k}\left(\mathbb{R}^{n}\right)$, then the trace of $\omega$ on $V$ belongs to $\mathcal{P}_{r}^{-} \Lambda^{k}(V)$.

\section{Polynomial differential forms on a simplex}

Having introduced the spaces of polynomial differential forms $\mathcal{P}_{r} \Lambda^{k}\left(\mathbb{R}^{n}\right)$ and $\mathcal{P}_{r}^{-} \Lambda^{k}\left(\mathbb{R}^{n}\right)$, we now wish to create finite element spaces of differential forms. These will be obtained using a triangulation of the domain and assembling spaces of polynomial differential forms on each of the simplices in the triangulation. First, for each simplex $T$ of the triangulation, we specify a space of shape functions. This will be either $\mathcal{P}_{r} \Lambda^{k}(T)$ or $\mathcal{P}_{r}^{-} \Lambda^{k}(T)$, where these denote the spaces of forms obtained by restricting the forms in $\mathcal{P}_{r} \Lambda^{k}\left(\mathbb{R}^{n}\right)$ and $\mathcal{P}_{r}^{-} \Lambda^{k}\left(\mathbb{R}^{n}\right)$, respectively, to $T$. It is also necessary to specify how these pieces are assembled to obtain a global space - in other words to specify the degree of interelement continuity. To this end, we need to specify a set of degrees of freedom for the shape spaces associated to a simplex - that is, a basis for the dual space - in which each degree of freedom is associated with a particular subsimplex. When a subsimplex is shared by more than one simplex in the triangulation, we will insist that the degrees of freedom associated with that subsimplex be single-valued, and this will determine the interelement continuity. This assembly process is an important part of the architecture of finite element codes, and the specification of a geometrically structured set of degrees of freedom distinguishes a finite element space from an arbitrary piecewise polynomial space. The association of the degrees of freedom to subsimplices gives a decomposition of the dual space of the shape functions on $T$ into a direct sum of subspaces indexed by all the subsimplices of $T$. It is really this geometric decomposition of the dual space that determines the interelement continuity rather than the particular choice of degrees of freedom, since we may choose any convenient basis for each space in the decomposition and obtain the same assembled finite element space.

For computation with finite elements we also need a basis, not only for the dual space, but also for the space of shape functions itself, which similarly decomposes the spaces into subspaces indexed by the subsimplices. This way the necessary discretization matrices can be computed simplex by simplex and assembled into a global matrix. In this section we define such bases and decompositions for $\mathcal{P}_{r} \Lambda^{k}(T)$ and $\mathcal{P}_{r}^{-} \Lambda^{k}(T)$. There have been several papers which have discussed bases of these spaces for use in computation, in particular cases, e.g., Webb (1999), Hiptmair (2001), Ainsworth and Coyle (2003), Gopalakrishnan, García-Castillo and Demkowicz (2005). In our 
presentation, we emphasize the fact, first noted in Arnold et al. (2006b), that the construction of the degrees of freedom and basis for the $\mathcal{P}_{r} \Lambda^{k}$ spaces requires the use $\mathcal{P}_{r}^{-} \Lambda^{k}$ spaces on the subsimplices, and vice versa. Therefore, the two families of spaces must be studied together to get optimal results.

\subsection{Simplices and barycentric coordinates}

For $1 \leq k \leq n$, let $\Sigma(k, n)$ denote the set of increasing maps $\{1, \ldots, k\} \rightarrow$ $\{1, \ldots, n\}$ and for $0 \leq k \leq n$, let $\Sigma_{0}(k, n)$ denote the set of increasing maps $\{0, \ldots, k\} \rightarrow\{0, \ldots, n\}$. These sets have cardinality $\left(\begin{array}{l}n \\ k\end{array}\right)$ and $\left(\begin{array}{l}n+1 \\ k+1\end{array}\right)$ respectively. For $\sigma \in \Sigma(k, n)$ with $k<n$ we define $\sigma^{*} \in \Sigma(n-k, n)$ such that $\mathcal{R}(\sigma) \cup \mathcal{R}\left(\sigma^{*}\right)=\{1, \ldots, n\}$ and, similarly, for $\sigma \in \Sigma_{0}(k, n)$ with $k<n$, we define $\sigma^{*} \in \Sigma_{0}(n-k-1, n)$ such that $\mathcal{R}(\sigma) \cup \mathcal{R}\left(\sigma^{*}\right)=\{0, \ldots, n\}$. For $\sigma \in \Sigma(k, n)$ we define $(0, \sigma) \in \Sigma_{0}(k, n)$ by $(0, \sigma)(0)=0,(0, \sigma)(j)=\sigma(j)$, $j=1, \ldots, k$.

Let $x_{0}, x_{1}, \ldots, x_{n}$ be $n+1$ points in general position in $\mathbb{R}^{n}$ ordered so that the vectors $x_{1}-x_{0}, \ldots, x_{n}-x_{0}$ give a positively oriented frame. Then the closed convex hull of these points, which we denote by $\left[x_{0}, \ldots, x_{n}\right]$, is the $n$-simplex with the vertices $x_{i}$. Call this simplex $T$. For each $\sigma \in \Sigma_{0}(k, n)$, the set $f_{\sigma}=\left[x_{\sigma(0)}, \ldots, x_{\sigma(k)}\right]$ is a subsimplex of dimension $k$. There are $\left(\begin{array}{l}n+1 \\ k+1\end{array}\right)$ subsimplices of dimension $k$, with the vertices being the subsimplices of dimension 0 , and $T$ itself being the only subsimplex of dimension $n$. For $k<n, f_{\sigma^{*}}$ is the $(n-k-1)$-dimensional subsimplex of $T$ opposite to the $k$-subsimplex $f_{\sigma}$. We denote the set of subsimplices of dimension $k$ of $T$ by $\Delta_{k}(T)$, and the set of all subsimplices of $T$ by $\Delta(T)$.

We denote by $\lambda_{0}, \ldots, \lambda_{n}$ the barycentric coordinate functions, so $\lambda_{i} \in$ $\mathcal{P}_{1}\left(\mathbb{R}^{n}\right)$ is determined by the equations $\lambda_{i}\left(x_{j}\right)=\delta_{i j}, 0 \leq i, j \leq n$. The $\lambda_{i}$ form a basis for $\mathcal{P}_{1}\left(\mathbb{R}^{n}\right)$ and satisfy $\sum_{i} \lambda_{i} \equiv 1$. We have

$$
T=\left\{x \in \mathbb{R}^{n} \mid \lambda_{i}(x) \geq 0, \quad i=0, \ldots, n\right\},
$$

and for the subsimplices

$$
f_{\sigma}=\left\{x \in T \mid \lambda_{i}(x)=0, i \in \mathcal{R}\left(\sigma^{*}\right)\right\} .
$$

In particular, the subsimplices of codimension one, or faces, of $T$ are

$$
F_{i}:=\left[x_{0}, \ldots, \hat{x}_{i}, \ldots, x_{n}\right]=\left\{x \in T \mid \lambda_{i}(x)=0\right\}, \quad i=0, \ldots, n .
$$

For a subsimplex $f=f_{\sigma}$, the functions $\lambda_{\sigma(0)}, \ldots, \lambda_{\sigma(k)}$ are the barycentric coordinates of $f$. Note that they are defined on all of $\mathbb{R}^{n}$. Their restrictions to $f$ depends only on $f$, but their values off $f$ depend on all the vertices of $T$. There is an isomorphism between the space $\mathcal{P}_{r}(f)$ of polynomial functions on $f$ of degree at most $r$, i.e., the restrictions of functions in $\mathcal{P}_{r}\left(\mathbb{R}^{n}\right)$ to $f$, and the space $\mathcal{H}_{r}\left(\mathbb{R}^{k+1}\right)$ of homogeneous polynomials of degree $r$ in $k+1$ 
variables. Namely each $p \in \mathcal{P}_{r}(f)$ may be expressed as

$$
p(x)=q\left(\lambda_{\sigma(0)}(x), \ldots, \lambda_{\sigma(k)}(x)\right), \quad x \in f,
$$

for a unique $q \in \mathcal{H}_{r}\left(\mathbb{R}^{k+1}\right)$. The right-hand side is defined for all $x \in \mathbb{R}^{n}$, and so provides a way to extend functions from $\mathcal{P}_{r}(f)$ to $\mathcal{P}_{r}\left(\mathbb{R}^{n}\right)$. We shall call this extension $E_{f, T}(p)$. Note that $E_{f, T}(p)$ vanishes on all subsimplices of $T$ disjoint from $f$.

The bubble function associated to a subsimplex $f=f_{\sigma}$ of $T$ is given by the product

$$
b_{f}=\lambda_{\sigma}:=\lambda_{\sigma(0)} \lambda_{\sigma(1)} \cdots \lambda_{\sigma(k)} \in \mathcal{P}_{k}\left(\mathbb{R}^{n}\right) .
$$

It vanishes on any subsimplex of $T$ which does not contain $f$, and, in particular, on all the subsimplices of dimension less than $k$. Thus its restriction to $f$ belongs to $\stackrel{\circ}{\mathcal{P}}_{k+1}(f)$, the subspace of $\mathcal{P}_{k+1}(f)$ consisting of polynomials functions on $f$ which vanish on the boundary of $f$.

The vectors $t_{i}:=x_{i}-x_{0}, i=1, \ldots, n$, form a basis for $\mathbb{R}^{n}$. The dual basis functions are the 1 -forms $\mathrm{d} \lambda_{i} \in \mathrm{Alt}^{1} \mathbb{R}^{n}$. Note that $\mathrm{d} \lambda_{0}=-\sum_{i=1}^{n} \mathrm{~d} \lambda_{i}$ is not included in the basis. Similarly, for any face $f=f_{\sigma}$ the restrictions of $\mathrm{d} \lambda_{\sigma(1)}, \ldots, \mathrm{d} \lambda_{\sigma(k)}$ to the tangent space $V$ of $f$ at any point of $f(V$ is independent of the point), give a basis for $\operatorname{Alt}^{1} V$.

The algebraic $k$-forms $(\mathrm{d} \lambda)_{\sigma}:=\mathrm{d} \lambda_{\sigma(1)} \wedge \cdots \wedge \mathrm{d} \lambda_{\sigma(k)}, \sigma \in \Sigma(k, n)$, form a basis for $\mathrm{Alt}^{k}$. Therefore, a differential $k$-form $\omega$ can be expressed in the form

$$
\omega=\sum_{\sigma \in \Sigma(k, n)} a_{\sigma}(\mathrm{d} \lambda)_{\sigma},
$$

for some coefficient functions $a_{\sigma}: T \rightarrow \mathbb{R}$. The coefficient functions in this expansion are uniquely determined and can be recovered using the dual basis:

$$
a_{\sigma}(x)=\omega_{x}\left(t_{\sigma(1)}, \ldots, t_{\sigma(k)}\right) .
$$

For any simplex $f$, let $|f|=\int_{f}$ vol $_{f}$ denote its $k$-dimensional volume. Then $\operatorname{vol}_{T}\left(t_{1}, \ldots, t_{n}\right)=n !|T|$, while $\mathrm{d} \lambda_{1} \wedge \cdots \wedge \mathrm{d} \lambda_{n}\left(t_{1}, \ldots, t_{n}\right)=1$. Thus $\mathrm{d} \lambda_{1} \wedge \cdots \wedge \mathrm{d} \lambda_{n}=1 /(n !|T|)$ vol $_{T}$. Similarly, if $f$ is the $k$-simplex with vertices $x_{\sigma(0)}, \ldots, x_{\sigma(k)}$, then

$$
\mathrm{d} \lambda_{\sigma(1)} \wedge \cdots \wedge \mathrm{d} \lambda_{\sigma(k)}= \pm \frac{1}{k !|f|} \operatorname{vol}_{f} .
$$

It follows that

$$
\int_{f} \lambda_{\sigma(j)} \mathrm{d} \lambda_{\sigma(1)} \wedge \cdots \wedge \mathrm{d} \lambda_{\sigma(k)}= \pm \frac{1}{k !|f|} \int_{f} \lambda_{\sigma(j)} \operatorname{vol}_{f}=\frac{1}{(k+1) !}, j=0, \ldots, k .
$$




\subsection{Degrees of freedom and basis for $\mathcal{P}_{r}(T)$}

Before proceeding to the case of general $\mathcal{P}_{r} \Lambda^{k}(T)$ and $\mathcal{P}_{r}^{-} \Lambda^{k}(T)$, we consider some simple cases. First we consider the familiar case of $\mathcal{P}_{r}(T)=\mathcal{P}_{r} \Lambda^{0}(T)=$ $\mathcal{P}_{r}^{-} \Lambda^{0}(T)$, which will correspond to the Lagrange finite element spaces.

It is well known (and is easily shown, and will follow from the more general result below) that an element of $\mathcal{P}_{r}(T)$ vanishes if it vanishes at the vertices, its moments of degree at most $r-2$ vanish on each edge, its moments of degree at most $r-3$ vanish on each 2 -subsimplex, etc.Let us associate to each $f \in \Delta(T)$, a subspace of the dual space of $\mathcal{P}_{r}(T)^{*}$ by

$$
W(f):=\left\{\phi \in \mathcal{P}_{r}(T)^{*} \mid \phi(p)=\int_{f} p q \operatorname{vol}_{f} \text { for some } q \in \mathcal{P}_{r-\operatorname{dim} f-1}(f)\right\} .
$$

In case $\operatorname{dim} f=0$, i.e, $f=\left\{x_{i}\right\}$ for some vertex $x_{i}$, then $\mathcal{P}_{r}(f)=\mathbb{R}$ and $\int_{f} p \mathrm{vol}_{f}=p\left(x_{i}\right)$, so $W(f)$ is the span of this evaluation functional at the vertex. Thus if $\phi(p)=0$ for all $p \in \sum_{f} W(f)$, then $p$ vanishes. Therefore, the $W(f) \operatorname{span} \mathcal{P}_{r}(T)^{*}$, i.e., $\sum_{f \in \Delta(f)} W(f)=\mathcal{P}_{r}(T)^{*}$. Obviously $\mathcal{P}_{r-\operatorname{dim} f-1}(f)$ maps onto $W(f)$ for each $f$. But it is easy to check that

$$
\sum_{f \in \Delta(T)} \operatorname{dim} \mathcal{P}_{r-\operatorname{dim} f-1}(f)=\operatorname{dim} \mathcal{P}_{r}(T)^{*},
$$

from which we conclude that $\mathcal{P}_{r-\operatorname{dim} f-1}(f) \cong W(f)$ and

$$
\mathcal{P}_{r}(T)^{*}=\bigoplus_{f \in \Delta(T)} W(f)
$$

This is the desired geometrical decomposition of the dual space of $\mathcal{P}_{r}(T)$. As mentioned, it is then easy to generate a set of degrees of freedom by choosing a convenient basis for each of the spaces $\mathcal{P}_{r-\operatorname{dim} f-1}(f)$, for example the monomials of degree $r-\operatorname{dim} f-1$ in the barycentric coordinates for $f$.

Next we give the geometrical decomposition of $\mathcal{P}_{r}(T)$ itself. We start with the monomial basis in barycentric coordinates (sometimes called the Bernstein basis): the basis functions are the polynomials $\lambda^{I}=\lambda_{0}^{i_{0}} \cdots \lambda_{n}^{i_{n}}$ where $I=\left(i_{0}, \ldots, i_{n}\right) \in \mathbb{N}^{n+1}$ is a multi-index for which the $i_{j}$ sum to $r$. We then associate the monomial $\lambda^{I}$ to the simplex $f$ whose vertices are the $x_{j}$ for which $i_{j}>0$. Thus $\lambda_{0}^{r}$ is associated to the vertex $x_{0}$, the $r-1$ monomials $\lambda_{0}^{r-1} \lambda_{1}, \lambda_{0}^{r-2} \lambda_{1}^{2}, \ldots, \lambda_{0} \lambda_{1}^{r-1}$ are associated to the edge $\left[x_{0}, x_{1}\right]$, etc.

Define $V(f)$ to be the span of the monomials so associated with $f$. Obviously

$$
\mathcal{P}_{r}(T)=\bigoplus_{f \in \Delta(T)} V(f)
$$


and it is easy to see that $\mathcal{P}_{r-\operatorname{dim} f-1}(f) \cong V(f)=E_{f, T} \stackrel{\circ}{\mathcal{P}}_{r}(f)$ with the isomorphism given by $p \mapsto b_{f} E_{f, T}(p)$.

\subsection{Degrees of freedom and basis for the Whitney forms}

A differential $k$-form on $T$ can be integrated over a $k$-simplex $f \in \Delta_{k}(T)$ and thus associates to each such $f$ a real number. (In the language of algebraic topology, a differential form determines a simplicial $k$-cochain.) Given any simplicial $k$-cochain, i.e., any choice of real numbers, one for each $f \in$ $\Delta_{k}(T)$, Whitney showed how to define a differential form corresponding to that cochain. Namely, he associated to $f=f_{\sigma} \in \Delta_{k}(T)$ a differential $k$-form on $T$, which we shall call the Whitney form associated to the subsimplex $f$, given by

$$
\phi_{\sigma}:=\sum_{i=0}^{k}(-1)^{i} \lambda_{\sigma(i)} \mathrm{d} \lambda_{\sigma(0)} \wedge \cdots \wedge \widehat{\mathrm{d}}_{\sigma(i)} \wedge \cdots \wedge \mathrm{d} \lambda_{\sigma(k)}
$$

(Whitney 1957, equation (12), p. 139). Now if $f^{\prime}=f_{\rho}$ is a $k$-subsimplex different from $f$, then for some $i, \sigma(i) \notin \mathcal{R}(\rho)$, and so the trace of $\lambda_{\sigma(i)}$ and $\mathrm{d} \lambda_{\sigma(i)}$ both vanish on $f^{\prime}$. Thus $\operatorname{Tr}_{f^{\prime}} \phi_{\sigma}=0$. On the other hand, it follows from (4.2) that $\int_{f} \phi_{\sigma}= \pm 1 / k$ !. Thus the Whitney $k$-forms corresponding to the $k$-subsimplices of $T$ span a subspace of $\mathcal{P}_{1} \Lambda^{k}(T)$ which is isomorphic to the space of $k$-dimensional simplicial cochains.

The next result asserts that the space spanned by the Whitney $k$-forms is precisely the space $\mathcal{P}_{1}^{-} \Lambda^{k}(T)$.

Theorem 4.1. The Whitney $k$-forms $\phi_{\sigma}$ corresponding to $f_{\sigma} \in \Delta_{k}(T)$ form a basis for $\mathcal{P}_{1}^{-} \Lambda^{k}(T)$.

Proof. It is enough to show that $\phi_{\sigma} \in \mathcal{P}_{1}^{-} \Lambda^{k}(T)$, since we have already seen that the $\phi_{\sigma}$ are linearly independent, and that their number equals $\left(\begin{array}{l}n+1 \\ k+1\end{array}\right)=\operatorname{dim} \mathcal{P}_{1}^{-} \Lambda^{k}(T)$; see (3.15). Now for each $i, \kappa \mathrm{d} \lambda_{i}=\lambda_{i}-\lambda_{i}(0)$, so $\kappa \mathrm{d} \lambda_{i}$ differs from $\lambda_{i}$ by a constant. Combining this with the Leibniz rule for $\kappa$ (3.6) we conclude that $\kappa\left(\mathrm{d} \lambda_{\sigma(0)} \wedge \cdots \mathrm{d} \lambda_{\sigma(k)}\right)$ differs from $\phi_{\sigma}$ by a constant $k$-form. Thus $\phi_{\sigma} \in \mathcal{P}_{0} \Lambda^{k}(T)+\kappa \mathcal{P}_{0} \Lambda^{k+1}(T)=\mathcal{P}_{1}^{-} \Lambda^{k}(T)$.

The trace of a function in $\mathcal{P}_{1}^{-} \Lambda^{k}(T)$ on a $k$-dimensional face $f$ belongs to $\mathcal{P}_{1}^{-} \Lambda^{k}(f)=\mathcal{P}_{0} \Lambda^{k}(f)$, a 1-dimensional space. Thus, for $f=f_{\sigma}, \operatorname{Tr}_{f} \phi_{\sigma}$ is a nonzero constant multiple of vol $_{f}$, while on the other $k$-dimensional subsimplices the trace of $\phi_{\sigma}$ vanishes.

Based on the Whitney forms, we again have geometrical decompositions

$$
\mathcal{P}_{1}^{-} \Lambda^{k}(T)=\bigoplus_{f \in \Delta(T)} V(f), \quad \mathcal{P}_{1}^{-} \Lambda^{k}(T)^{*}=\bigoplus_{f \in \Delta(T)} W(f),
$$

where now $V(f)$ and $W(f)$ vanish unless $\operatorname{dim} f=k$ and for $f=f_{\sigma} \in \Delta_{k}(T)$, 
$V(f)$ and $W(f)$ are 1-dimensional:

$$
V(f)=\mathbb{R} \phi_{\sigma}, \quad W(f)=\mathbb{R}\left[\int_{f} \operatorname{Tr}_{f}(\cdot)\right] .
$$

\subsection{A basis for $\mathcal{P}_{r}^{-} \Lambda^{k}(T)$}

In this subsection we display a first basis for $\mathcal{P}_{r}^{-} \Lambda^{k}(T)$, analogous to the basis (4.1) for $\mathcal{P}_{r} \Lambda^{k}(T)$. This basis is not well adapted for numerical computation, because it does not admit an appropriate geometric decomposition. Such a basis, which is more difficult to obtain, will be constructed in Section 4.7. We begin with two lemmas.

Lemma 4.2. Let $x$ be a vertex of $T$. Then the Whitney forms corresponding to the $k$-subsimplices that contain $x$ are linearly independent over the ring of polynomials $\mathcal{P}(T)$.

Proof. Without loss of generality, we may assume that the vertex $x=x_{0}$, so we must prove that if

$$
\omega=\sum_{\substack{\sigma \in \Sigma_{0}(k, n) \\ \sigma(0)=0}} p_{\sigma} \phi_{\sigma}
$$

vanishes on $T$ for some polynomials $p_{\sigma} \in \mathcal{P}(T)$, then all the $a_{\sigma}=0$. From the definition (4.3), we see that if $\sigma(0)=0$, then

$$
\phi_{\sigma}\left(x_{0}\right)=\mathrm{d} \lambda_{\sigma(1)} \wedge \cdots \wedge \mathrm{d} \lambda_{\sigma(k)} .
$$

Thus the values of the Whitney forms $\phi_{\sigma}$ at the vertex $x_{0}$ form a basis for $\mathrm{Alt}^{1} \mathbb{R}^{n}$. By continuity, we conclude that there is a neighbourhood $N$ of $x_{0}$ such that for $x \in N$ the algebraic $k$-forms $\phi_{\sigma}(x), \sigma \in \Sigma(k, n), \sigma(0)=0$, are linearly independent. Since

$$
\sum_{\substack{\sigma \in \Sigma_{0}(k, n) \\ \sigma(0)=0}} p_{\sigma}(x) \phi_{\sigma}(x)=\omega(x)=0,
$$

we conclude that for each $\sigma, p_{\sigma}(x)$ vanishes for all $x \in N$, whence $p_{\sigma} \equiv 0$.

Lemma 4.3. Suppose that

$$
\omega=\sum_{\sigma \in \Sigma_{0}(k, n)} p_{\sigma} \phi_{\sigma}
$$

where

$$
p_{\sigma}(x)=a_{\sigma}\left(\lambda_{\sigma(0)}(x), \lambda_{\sigma(0)+1}(x), \ldots, \lambda_{n}(x)\right),
$$

for some polynomial $a_{\sigma}$ in $n-\sigma_{0}+1$ variables. If $\omega$ vanishes, then each of the $a_{\sigma}$ vanishes. 
Proof. If one of the $a_{\sigma} \neq 0$, choose $\rho \in \Sigma_{0}(k, n)$ such that $a_{\rho} \neq 0$ but $a_{\sigma}=0$ if $\sigma(0)>j:=\rho(0)$. Let $f=\left[x_{j}, x_{j+1}, \ldots, x_{n}\right]$. Then $\operatorname{Tr}_{f} \phi_{\sigma}=0$ if $\sigma(0)<j$ and $a_{\sigma}=0$ if $\sigma(0)>j$, so

$$
\operatorname{Tr}_{f} \omega=\sum_{\substack{\sigma \in \Sigma_{0}(k, n) \\ \sigma(0)=j}} \operatorname{Tr}_{f}\left(p_{\sigma}\right) \operatorname{Tr}_{f} \phi_{\sigma} .
$$

Now $\operatorname{Tr}_{f} \phi_{\sigma}$ is the Whitney form on $f$ associated to the subsimplex $f_{\sigma}$ of $f$, so the preceding lemma (applied to $f$ in place of $T$ ) implies that $\operatorname{Tr}_{f}\left(p_{\sigma}\right)=0$ for all $\sigma$ with $\sigma(0)=j$, and, in particular, for $\sigma=\rho$. Since $p_{\rho}=a_{\rho}\left(\lambda_{j}, \ldots, \lambda_{n}\right)$, this implies that $a_{\rho}=0$, a contradiction.

Theorem 4.4. For each $\sigma \in \Sigma_{0}(k, n)$ let $a_{\sigma} \in \mathcal{H}_{r-1}\left(\mathbb{R}^{n-\sigma(0)+1}\right)$. Then the $k$-form

$$
\omega=\sum_{\sigma \in \Sigma_{0}(k, n)} a_{\sigma}\left(\lambda_{\sigma(0)}, \lambda_{\sigma(0)+1}, \ldots, \lambda_{n}\right) \phi_{\sigma}
$$

belongs to $\mathcal{P}_{r}^{-} \Lambda^{k}(T)$. Moreover, each $\omega \in \mathcal{P}_{r}^{-} \Lambda^{k}(T)$ can be written in the form (4.4) for a unique choice of polynomials $a_{\sigma} \in \mathcal{H}_{r-1}\left(\mathbb{R}^{n-\sigma(0)+1}\right)$.

Proof. For $a_{\sigma} \in \mathcal{H}_{r-1}\left(\mathbb{R}^{n-\sigma(0)+1}\right), a_{\sigma}\left(\lambda_{\sigma(0)}, \lambda_{\sigma(0)+1}, \ldots, \lambda_{n}\right) \in \mathcal{P}_{r-1}(T)$, so $\omega \in \mathcal{P}_{r}^{-} \Lambda^{k}(T)$ by $(3.17)$.

Thus (4.4) defines a linear mapping

$$
\bigoplus_{\sigma \in \Sigma_{0}(k, n)} \mathcal{H}_{r-1}\left(\mathbb{R}^{n-\sigma(0)+1}\right) \rightarrow \mathcal{P}_{r}^{-} \Lambda^{k}(T) .
$$

By the preceding lemma, we know that this map is injective, so to complete the proof of the theorem it suffices to check that

$$
\sum_{\sigma \in \Sigma_{0}(k, n)} \operatorname{dim} \mathcal{H}_{r-1}\left(\mathbb{R}^{n-\sigma(0)+1}\right)=\operatorname{dim} \mathcal{P}_{r}^{-} \Lambda^{k}(T)=\left(\begin{array}{c}
r+k-1 \\
k
\end{array}\right)\left(\begin{array}{c}
n+r \\
r+k
\end{array}\right) .
$$

To see this, observe that for a fixed $j, 0 \leq j \leq n-k$, we have by (3.1)

$$
\begin{aligned}
& \sum_{\substack{\sigma \in \Sigma_{0}(k, n) \\
\sigma(0)=j}} \operatorname{dim} \mathcal{H}_{r-1}\left(\mathbb{R}^{n-\sigma(0)+1}\right) \\
& \quad=\operatorname{dim} \mathcal{P}_{r-1} \Lambda^{k}\left(\mathbb{R}^{n-j}\right)=\left(\begin{array}{c}
r+k-1 \\
k
\end{array}\right)\left(\begin{array}{c}
n-j+r-1 \\
r+k-1
\end{array}\right) .
\end{aligned}
$$

Therefore, we have

$$
\begin{aligned}
\sum_{\sigma \in \Sigma_{0}(k, n)} & \operatorname{dim} \mathcal{H}_{r-1}\left(\mathbb{R}^{n-\sigma(0)+1}\right) \\
& =\left(\begin{array}{c}
r+k-1 \\
k
\end{array}\right) \sum_{j=0}^{n-k}\left(\begin{array}{c}
n-j+r-1 \\
r+k-1
\end{array}\right)=\left(\begin{array}{c}
r+k-1 \\
k
\end{array}\right)\left(\begin{array}{c}
n+r \\
r+k
\end{array}\right) .
\end{aligned}
$$


Here, we have used the identity

$$
\sum_{j=0}^{s}\left(\begin{array}{c}
m+j \\
m
\end{array}\right)=\left(\begin{array}{c}
m+s+1 \\
m+1
\end{array}\right),
$$

which is easily established by induction on $s$.

Of course the theorem implies that we obtain a basis for $\mathcal{P}_{r}^{-} \Lambda^{k}(T)$, by choosing any convenient basis, e.g., the monomial basis, for each of the spaces $\mathcal{H}_{r-1}\left(\mathbb{R}^{n-\sigma(0)+1}\right)$.

\subsection{Geometrical decomposition of $\mathcal{P}_{r} \Lambda^{k}(T)^{*}$}

We now turn to the general case of $\mathcal{P}_{r} \Lambda^{k}(T)$ and $\mathcal{P}_{r}^{-} \Lambda^{k}(T)$, providing a geometrical decomposition of each space and its dual, as was done for $\mathcal{P}_{r} \Lambda^{0}(T)$ and $\mathcal{P}_{1}^{-} \Lambda^{k}(T)$ in Sections 4.2 and 4.3. In this subsection, we construct the decomposition of $\mathcal{P}_{r} \Lambda^{k}(T)^{*}$ in which the summand associated to a subsimplex is isomorphic to a space of polynomial differential forms (of the $\mathcal{P}_{s}^{-}$ type) on the subsimplex. As a consequence, we obtain (via choice of bases for the summands), a set of degrees of freedom for $\mathcal{P}_{r} \Lambda^{k}(T)$, with each degree of freedom associated to a subsimplex of $T$. This will allow the construction of finite element differential forms based on the $\mathcal{P}_{r} \Lambda^{k}$ spaces in the next section.

The decomposition, which is given in Theorem 4.10, will be built up in a sequence of results.

Lemma 4.5. An element $\omega \in \mathcal{P}_{r} \Lambda^{k}(T)$ has vanishing trace on the faces $F_{1}, \ldots, F_{n}$ (but not necessarily on $F_{0}$ ) if and only if it can be written in the form

$$
\omega=\sum_{\sigma \in \Sigma(k, n)} p_{\sigma} \lambda_{\sigma^{*}}(\mathrm{~d} \lambda)_{\sigma}
$$

for some $p_{\sigma} \in \mathcal{P}_{r-n+k}(T)$.

Proof. Both $\lambda_{i}$ and the trace of $\mathrm{d} \lambda_{i}$ vanish on the face $F_{i}$, so all the forms $\lambda_{\sigma^{*}}(\mathrm{~d} \lambda)_{\sigma}$ vanish on each $F_{i}, i=1, \ldots, n$. Thus if $\omega$ has the form (4.5), then its traces vanish as claimed.

On the other hand, suppose the $\omega \in \mathcal{P}_{r} \Lambda^{k}(T)$ has such vanishing traces. Write $\omega$ as in (4.1) with the $a_{\sigma} \in \mathcal{P}_{r}(T)$. We must show that $\lambda_{\sigma^{*}}$ is a divisor of $a_{\sigma}$. For $1 \leq i \leq k$, and $1 \leq j \leq n-k$, the vertices $x_{0}$, and $x_{\sigma(i)}$ belong to the face $F_{\sigma^{*}(j)}$. Therefore the vector $t_{\sigma(i)}$ is tangent to the face, and so

$$
p_{\sigma}(x)=\omega_{x}\left(t_{\sigma(1)}, \ldots, t_{\sigma(k)}\right)
$$

must vanish on $F_{\sigma^{*}(j)}$. Thus $\lambda_{\sigma^{*}(j)}$ divides $p_{\sigma}(x)$ for each $1 \leq j \leq n-k$, i.e., $\lambda_{\sigma^{*}}$ divides $p_{\sigma}$ as claimed. 
Lemma 4.6. Let $\omega \in \mathcal{P}_{r} \Lambda^{k}(T)$. Suppose that $\operatorname{Tr}_{F_{i}} \omega=0$ for $i=1, \ldots, n$ and that

$$
\int \omega \wedge \eta=0, \quad \eta \in \mathcal{P}_{r-n+k} \Lambda^{n-k}(T) .
$$

Then $\omega=0$.

Proof. Write $\omega$ as in (4.5), and set

$$
\eta=\sum_{\sigma \in \Sigma(k, n)}( \pm)_{\sigma} p_{\sigma}(\mathrm{d} \lambda)_{\sigma^{*}} \in \mathcal{P}_{r-n+k} \Lambda^{n-k}(T),
$$

where $( \pm)_{\sigma}$ is the sign of the permutation $\left(\sigma(1), \ldots, \sigma(k), \sigma^{*}(1), \ldots, \sigma^{*}(n-\right.$ $k)$ ). Then

$$
0=\int \omega \wedge \mu=\left(\sum_{\sigma} \int p_{\sigma}^{2} \lambda_{\sigma^{*}}\right) \mathrm{d} \lambda_{1} \wedge \cdots \wedge \mathrm{d} \lambda_{n} .
$$

Since $\lambda_{\sigma^{*}}>0$ on the interior of $T$, we conclude that all the $p_{\sigma} \equiv 0$ and so $\omega=0$.

Lemma 4.7. Let $\omega \in \stackrel{\circ}{\mathcal{P}}_{r} \Lambda^{k}(T)$. Suppose that

$$
\int \omega \wedge \eta=0, \quad \eta \in \mathcal{P}_{r-n+k}^{-} \Lambda^{n-k}(T) .
$$

Then $\omega=0$.

Proof. If $\omega \in \stackrel{\circ}{\mathcal{P}}_{r} \Lambda^{k}(T)$, then $\mathrm{d} \omega \in \stackrel{\circ}{\mathcal{P}}_{r-1} \Lambda^{k+1}(T)$, and

$$
\int \mathrm{d} \omega \wedge \mu= \pm \int \omega \wedge \mathrm{d} \mu, \quad \mu \in \Lambda^{n-k-1} .
$$

Now if $\mu \in \mathcal{P}_{r-n+k} \Lambda^{n-k-1}(T)$, then

$$
\mathrm{d} \mu \in \mathcal{P}_{r-n+k-1} \Lambda^{n-k}(T) \subset \mathcal{P}_{r-n+k}^{-} \Lambda^{n-k}(T),
$$

so

$$
\int \mathrm{d} \omega \wedge \mu=0, \quad \eta \in \mathcal{P}_{r-n+k} \Lambda^{n-k-1}(T) .
$$

Applying Lemma 4.6, we conclude that $\mathrm{d} \omega=0$, and hence, by (4.8), that

$$
\int \omega \wedge \eta=0, \quad \eta \in \Lambda^{n-k-1}(T) .
$$

Together with the hypothesis (4.7), we find that the hypothesis (4.6) of the previous lemma is fulfilled, and so $\omega$ vanishes.

Now for some $0 \leq k \leq n, r \geq 1$, let $\omega \in \mathcal{P}_{r} \Lambda^{k}(T)$ and $f \in \Delta_{k}(T)$. The trace of $\omega$ on $\partial f$ certainly vanishes (since it is a $k$-form on a manifold of 
dimension $k-1)$, so if $\int_{f} \operatorname{Tr}_{f} \omega \wedge \eta=0$ for all $\eta \in \mathcal{P}_{r}^{-} \Lambda^{0}(f)$, then, by the previous lemma applied to $f, \operatorname{Tr}_{f} \omega=0$. Therefore, if we assume that

$$
\int_{f} \operatorname{Tr}_{f} \omega \wedge \eta=0, \quad \eta \in \mathcal{P}_{r}^{-} \Lambda^{0}(f), \quad f \in \Delta_{k}(T)
$$

we conclude that $\operatorname{Tr}_{f} \omega=0$ for all $f \in \Delta_{k}(T)$. If we then assume also that

$$
\int_{f} \operatorname{Tr}_{f} \omega \wedge \eta=0, \quad \eta \in \mathcal{P}_{r-1}^{-} \Lambda^{1}(f), \quad f \in \Delta_{k+1}(T),
$$

we may apply the lemma again to conclude that $\operatorname{Tr}_{f} \omega=0$ for all $f \in$ $\Delta_{k+1}(T)$. Continuing in this way, we obtain the following theorem.

Theorem 4.8. Let $0 \leq k \leq n, r \geq 1$. Suppose that $\omega \in \mathcal{P}_{r} \Lambda^{k}(T)$ satisfies

$$
\int_{f} \operatorname{Tr}_{f} \omega \wedge \eta=0, \quad \eta \in \mathcal{P}_{r+k-\operatorname{dim} f}^{-} \Lambda^{\operatorname{dim} f-k}(f), \quad f \in \Delta(T) .
$$

Then $\omega=0$.

Note that since $\mathcal{P}_{s}^{-} \Lambda^{k}$ vanishes, if $s \leq 1$ or $k<0$, the only subsimplices that contribute in (4.9) have $k \leq \operatorname{dim} f \leq \min (n, r+k-1)$.

The association of $\eta$ to the linear functional $\omega \mapsto \int_{f} \operatorname{Tr}_{f} \omega \wedge \eta$, gives a surjection of $\mathcal{P}_{r+k-\operatorname{dim} f}^{-} \Lambda^{\operatorname{dim} f-k}(f)$ onto

$$
\begin{aligned}
W(f):=\left\{\phi \in \mathcal{P}_{r} \Lambda^{k}(T)^{*} \mid\right. & \\
& \left.\phi(\omega)=\int_{f} \operatorname{Tr}_{f} \omega \wedge \eta \text { for some } \eta \in \mathcal{P}_{r+k-\operatorname{dim} f}^{-} \Lambda^{\operatorname{dim} f-k}(f)\right\} .
\end{aligned}
$$

The following theorem, a simple dimension count, will imply that all these surjections are isomorphisms, and that the $W(f)$ span.

Theorem 4.9. Let $0 \leq k \leq n, r \geq 1$. Then

$$
\sum_{f \in \Delta(T)} \operatorname{dim} \mathcal{P}_{r+k-\operatorname{dim} f}^{-} \Lambda^{\operatorname{dim} f-k}(f)=\operatorname{dim} \mathcal{P}_{r} \Lambda^{k}(T)
$$

Proof. Using the convention that $\left(\begin{array}{c}m \\ l\end{array}\right)=0$ for $l<0$ or $l>m$, the fact that the number of simplices of dimension $j$ is $\left(\begin{array}{c}n+1 \\ j+1\end{array}\right)$, and the dimension formula (3.15), we see that the left-hand side equals

$$
\begin{aligned}
& \sum_{j}\left(\begin{array}{c}
n+1 \\
j+1
\end{array}\right)\left(\begin{array}{c}
r-1 \\
j-k
\end{array}\right)\left(\begin{array}{c}
r+k \\
k
\end{array}\right) \\
& =\left(\begin{array}{c}
r+k \\
k
\end{array}\right) \sum_{j=0}^{n-k}\left(\begin{array}{c}
n+1 \\
j+k+1
\end{array}\right)\left(\begin{array}{c}
r-1 \\
j
\end{array}\right)
\end{aligned}
$$




$$
\begin{aligned}
& =\left(\begin{array}{c}
r+k \\
k
\end{array}\right) \sum_{j=0}^{n-k}\left(\begin{array}{c}
n+1 \\
n-k-j
\end{array}\right)\left(\begin{array}{c}
r-1 \\
j
\end{array}\right) \\
& =\left(\begin{array}{c}
r+k \\
k
\end{array}\right)\left(\begin{array}{l}
n+r \\
n-k
\end{array}\right)=\operatorname{dim} \mathcal{P}_{r} \Lambda^{k}(T) .
\end{aligned}
$$

In the last step we have used the binomial identity

$$
\sum_{j}\left(\begin{array}{c}
l \\
p-j
\end{array}\right)\left(\begin{array}{c}
m \\
j
\end{array}\right)=\left(\begin{array}{c}
l+m \\
p
\end{array}\right),
$$

which can be deduced by matching coefficients of $x^{p}$ after binomial expansion of the equation $(x+1)^{l}(x+1)^{m}=(x+1)^{l+m}$.

Combining Theorems 4.8 and 4.11 , we have the main result of this subsection.

Theorem 4.10. Let $0 \leq k \leq n, r \geq 1$. For each $f \in \Delta(T)$, define $W(f)$ by (4.10). (Note that $W(f)=0$ unless $k \leq \operatorname{dim} f \leq \min (n, r+k-1)$.) Then $W(f) \cong \mathcal{P}_{r+k-\operatorname{dim} f}^{-} \Lambda^{\operatorname{dim} f-k}(f)$ and

$$
\mathcal{P}_{r} \Lambda^{k}(T)^{*}=\bigoplus_{f \in \Delta(T)} W(f) .
$$

We also have, as a corollary of the above, an isomorphism of $\stackrel{\circ}{\mathcal{P}}_{r} \Lambda^{k}(T)^{*}$ with $\mathcal{P}_{r+k-n}^{-} \Lambda^{n-k}(T)$; see Lemma 4.7. Thus

$$
\operatorname{dim} \stackrel{\circ}{\mathcal{P}}_{r} \Lambda^{k}(T)^{*}=\left(\begin{array}{c}
r-1 \\
n-k
\end{array}\right)\left(\begin{array}{c}
r+k \\
k
\end{array}\right) .
$$

\subsection{Geometrical decomposition of $\mathcal{P}_{r}^{-} \Lambda^{k}(T)^{*}$}

In a very similar manner, we obtain a geometrical decomposition of the dual of $\mathcal{P}_{r}^{-} \Lambda^{k}(T)$. First we prove an analogue of Lemma 4.7.

Lemma 4.11. Let $\omega \in \stackrel{\circ}{\mathcal{P}}_{r}^{-} \Lambda^{k}(T)$. Suppose that

$$
\int \omega \wedge \eta=0, \quad \eta \in \mathcal{P}_{r-n+k-1} \Lambda^{n-k}(T) .
$$

Then $\omega=0$.

Proof. $\quad$ Since $\omega \in \stackrel{\circ}{\mathcal{P}}_{r}^{-} \Lambda^{k}(T), \mathrm{d} \omega \in \stackrel{\circ}{\mathcal{P}}_{r-1} \Lambda^{k+1}(T)$. Therefore

$$
\int \mathrm{d} \omega \wedge \mu= \pm \int \omega \wedge \mathrm{d} \mu, \quad \mu \in \Lambda^{n-k-1} .
$$

Now if $\mu \in \mathcal{P}_{r-n+k} \Lambda^{n-k-1}(T)$, then $\mathrm{d} \mu$ belongs to $\mathcal{P}_{r-n+k-1} \Lambda^{n-k}(T)$ which is contained in $\mathcal{P}_{r-n+k}^{-} \Lambda^{n-k}(T)$, so $\int \mathrm{d} \omega \wedge \mu=0$. Thus $\int \mathrm{d} \omega \wedge \mu=0$ for $\mu \in \mathcal{P}_{r-n+k} \Lambda^{n-k-1}(T)$. Applying Lemma 4.6 with $r$ replaced by $r-1$ and 
$k$ replaced by $k+1$, we conclude that $\mathrm{d} \omega=0$. Theorem 3.4 then implies that $\omega \in \mathcal{P}_{r-1} \Lambda^{k}(T)$, and we can apply Lemma 4.6 again, this time with $r$ replaced by $r-1$, to conclude that $\omega$ vanishes.

Just as for Theorem 4.8, a finite induction based on this result gives the desired decomposition.

Theorem 4.12. Let $0 \leq k \leq n, r \geq 1$. Suppose that $\omega \in \mathcal{P}_{r}^{-} \Lambda^{k}(T)$ satisfies

$$
\int_{f} \operatorname{Tr}_{f} \omega \wedge \eta=0, \quad \eta \in \mathcal{P}_{r+k-\operatorname{dim} f-1} \Lambda^{\operatorname{dim} f-k}(f), \quad f \in \Delta(T) .
$$

Then $\omega=0$.

Note that since $\mathcal{P}_{s}^{-} \Lambda^{k}$ vanishes if $s \leq 1$ or $k<0$, again, the only subsimplices that contribute have $k \leq \operatorname{dim} f \leq \min (n, r+k-1)$.

The dimension count is a simple calculation like that of Theorem 4.9.

Theorem 4.13. Let $0 \leq k \leq n, r \geq 1$. Then

$$
\sum_{f \in \Delta(T)} \operatorname{dim} \mathcal{P}_{r+k-\operatorname{dim} f-1} \Lambda^{\operatorname{dim} f-k}(f)=\operatorname{dim} \mathcal{P}_{r}^{-} \Lambda^{k}(T) .
$$

Combining Theorems 4.12 and 4.12, we get the desired decomposition.

Theorem 4.14. Let $0 \leq k \leq n, r \geq 1$. For each $f \in \Delta(T)$, define

$$
\begin{aligned}
W(f):=\left\{\phi \in \mathcal{P}_{r}^{-} \Lambda^{k}(T)^{*} \mid\right. & \\
& \left.\phi(\omega)=\int_{f} \operatorname{Tr}_{f} \omega \wedge \eta \text { for some } \eta \in \mathcal{P}_{r+k-\operatorname{dim} f-1} \Lambda^{\operatorname{dim} f-k}(f)\right\} .
\end{aligned}
$$

(Note that $W(f)=0$ unless $k \leq \operatorname{dim} f \leq \min (n, r+k-1)$.) Then $W(f) \cong$ $\mathcal{P}_{r+k-\operatorname{dim} f-1} \Lambda^{\operatorname{dim} f-k}(f)$ and

$$
\mathcal{P}_{r}^{-} \Lambda^{k}(T)^{*}=\bigoplus_{f \in \Delta(T)} W(f) .
$$

Moreover, $\stackrel{\circ}{\mathcal{P}}_{r}^{-} \Lambda^{k}(T)^{*} \cong \mathcal{P}_{r+k-n-1} \Lambda^{n-k}(T)$; see Lemma 4.11. Therefore,

$$
\operatorname{dim} \stackrel{\circ}{\mathcal{P}}_{r}^{-} \Lambda^{k}(T)=\left(\begin{array}{l}
n \\
k
\end{array}\right)\left(\begin{array}{c}
r+k-1 \\
n
\end{array}\right) \text {. }
$$

\subsection{Geometrical decomposition of $\mathcal{P}_{r}^{-} \Lambda^{k}$}

Finally we give geometrical decompositions of the spaces themselves. From these we can easily obtain bases for use in computation. In this subsection we consider the spaces $\mathcal{P}_{r}^{-} \Lambda^{k}(T)$, and in the next, the spaces $\mathcal{P}_{r} \Lambda^{k}(T)$. Since we have already treated the case $k=0$, we assume that $1 \leq k \leq n$.

Our main result will be the following decomposition. 
Theorem 4.15. Let $k, r \geq 1$. Then

$$
\mathcal{P}_{r}^{-} \Lambda^{k}(T)=\bigoplus_{f \in \Delta(T)} V(f),
$$

where $V(f)=0$ if $\operatorname{dim} f<k$ or $\operatorname{dim} f \geq r+k$ and

$$
V(f) \cong \mathcal{P}_{r+k-\operatorname{dim} f-1} \Lambda^{\operatorname{dim} f-k}(f)
$$

otherwise.

We will build up to the proof with a number of preliminary results. First we obtain a representation of forms in $\mathcal{P}_{r}^{-} \Lambda^{k}(T)$ with vanishing trace. In it we use the notation $(0, \rho)$ to denote the extension of a sequence $\rho:\{1, \ldots, k\} \rightarrow\{1, \ldots, n\}$ to the sequence $(0, \rho):\{0, \ldots, k\} \rightarrow\{0, \ldots, n\}$ determined by $(0, \rho)(0)=0$. Note that for any $\rho \in \Sigma(k, n)$ the elements $\lambda_{\rho^{*}} \phi_{(0, \rho)}$ of $\mathcal{P}_{n-k+1}^{-} \Lambda^{k}(T)$ have vanishing trace.

Theorem 4.16. For $1 \leq k \leq n, r \geq n+1-k$, the map

$$
\sum_{\rho \in \Sigma(k, n)} a_{\rho}(\mathrm{d} \lambda)_{\rho^{*}} \mapsto \sum_{\rho \in \Sigma(k, n)} a_{\rho} \lambda_{\rho^{*}} \phi_{(0, \rho)},
$$

where the $a_{\rho} \in \mathcal{P}_{r+k-n-1}(T)$, defines an isomorphism of $\mathcal{P}_{r+k-n-1} \Lambda^{n-k}(T)$ onto $\stackrel{\circ}{\mathcal{P}}_{r}^{-} \Lambda^{k}(T)$.

Proof. The map is an injection according to Lemma 4.2, and Theorem 4.12 implies that $\operatorname{dim} \stackrel{\circ}{\mathcal{P}}_{r}^{-} \Lambda^{k}(T) \leq \operatorname{dim} \mathcal{P}_{r+k-n-1} \Lambda^{n-k}(T)$. The result follows.

Corollary 4.17. Let $f=f_{\sigma} \in \Delta(T)$ with $k \leq \operatorname{dim} f \leq r+k-1$. Then the map

$$
\sum_{\rho \in \Sigma(k, \operatorname{dim} f)} a_{\rho}(\mathrm{d} \lambda)_{\sigma \circ \rho^{*}} \mapsto \sum_{\rho \in \Sigma(k, \operatorname{dim} f)} a_{\rho} \lambda_{\sigma \circ \rho^{*}} \phi_{\sigma \circ(0, \rho)},
$$

where the $a_{\rho} \in \mathcal{P}_{r+k-\operatorname{dim} f-1}(f)$, defines an isomorphism

$$
\mathcal{P}_{r+k-\operatorname{dim} f-1} \Lambda^{\operatorname{dim} f-k}(f) \cong \stackrel{\circ}{\mathcal{P}}_{r}^{-} \Lambda^{k}(f) .
$$

Proof. This is just the theorem applied to $f$ rather than $T$.

From the unique representation

$$
\omega=\sum_{\rho \in \Sigma(k, \operatorname{dim} f)} a_{\rho} \lambda_{\sigma \circ \rho^{*}} \phi_{\sigma \circ(0, \rho)},
$$

we obtain an extension operator

$$
E_{f, T}^{k}: \stackrel{\mathcal{P}}{r}_{r}^{-} \Lambda^{k}(f) \rightarrow \mathcal{P}_{r}^{-} \Lambda^{k}(T) .
$$

Namely, we write the coefficients $a_{\rho}$ in terms of the barycentric coordinates 
on $f$, so the entire expression is in terms of these barycentric coordinates, and so extends to the whole of $\mathbb{R}^{n}$. We then obviously have

$$
\operatorname{Tr}_{f}\left(E_{f, T}^{k} \omega\right)=\omega, \quad \omega \in \stackrel{\circ}{\mathcal{P}}_{r}^{-} \Lambda^{k}(f) .
$$

Proposition 4.18. If $\omega \in \stackrel{\circ}{\mathcal{P}}_{r}^{-} \Lambda^{k}(f)$, then $\operatorname{Tr}_{F}\left(E_{f, T}^{k} \omega\right)$ vanishes for each face $F$ opposite a vertex on $f$.

Proof. Let $x_{i}$ be a vertex of $f$ and $F$ the face opposite (on which $\lambda_{i}=0$ ). For each $\rho \in \Sigma(k, \operatorname{dim} f), i$ is either in $\mathcal{R}\left(\sigma \circ \rho^{*}\right)$ or in $\mathcal{R}(\sigma \circ(0, \rho))$. In the first case, $\operatorname{Tr}_{F}\left(\lambda_{\sigma \circ \rho^{*}}\right)$ vanishes and in the latter, $\operatorname{Tr}_{F}\left(\phi_{\sigma \circ(0, \rho)}\right)$ vanishes. Thus each term in the expression for $E_{f, T}^{k} \omega$ has vanishing trace on $F$.

Corollary 4.19. Let $f, g \in \Delta(T)$ with dimensions at least $k$. If $f \nsubseteq g$, then

$$
\operatorname{Tr}_{g}\left(E_{f, T}^{k} \omega\right)=0, \quad \omega \in \stackrel{\circ}{\mathcal{P}}_{r}^{-} \Lambda^{k}(f) .
$$

Proof. There exists a vertex of $f$ which does not belong to $g$, and so $g$ is contained in the face opposite this vertex.

Proposition 4.20. For each $f \in \Delta(T)$ with $\operatorname{dim} f \geq k$, let $\omega_{f} \in \stackrel{\circ}{\mathcal{P}}_{r}^{-} \Lambda^{k}(f)$. If

$$
\sum_{\substack{f \in \Delta(T) \\ \operatorname{dim} f \geq k}} E_{f, T}^{k} \omega_{f}=0
$$

then each $\omega_{f}=0$.

Proof. If some $\omega_{f}$ does not vanish, there is a face $g$ of minimal dimension such that $\omega_{g} \neq 0$, so that $g$ does not contain any of the other faces $f$ for which $\omega_{f} \neq 0$. Taking the trace of the sum on $g$ and invoking the corollary, we conclude that $\omega_{g}=0$, a contradiction.

We can now prove Theorem 4.15. We define

$$
V(f)=E_{f, T}^{k} \stackrel{\circ}{\mathcal{P}}_{r}^{-} \Lambda^{k}(f),
$$

for $f$ with $k \leq \operatorname{dim} f<r+k$. By the previous proposition, the sum of the $V(f)$ is direct, and we need only prove that it equals all of $\mathcal{P}_{r}^{-} \Lambda^{k}(T)$. But we have already shown, in Theorem 4.14, that the dimensions of $V(f)$ sum to the dimension of $\mathcal{P}_{r}^{-} \Lambda^{k}(T)$, and this completes the proof.

\subsection{Geometrical decomposition of $\mathcal{P}_{r} \Lambda^{k}$}

In complete analogy with Theorem 4.15 above, we will establish a corresponding geometrical decomposition for the spaces $\mathcal{P}_{r} \Lambda^{k}(T)$. 
Theorem 4.21. Let $k, r \geq 1$. Then

$$
\mathcal{P}_{r} \Lambda^{k}(T)=\bigoplus_{f \in \Delta(T)} V(f)
$$

where $V(f)=0$ if $\operatorname{dim} f<k$ or $\operatorname{dim} f \geq r+k$ and

$$
V(f) \cong \mathcal{P}_{r+k-\operatorname{dim} f}^{-} \Lambda^{\operatorname{dim} f-k}(f)
$$

otherwise.

As above, we start the construction by defining an isomorphism onto the subspace of forms in $\mathcal{P}_{r} \Lambda^{k}(T)$ with vanishing trace, $\mathcal{P}_{r} \Lambda^{k}(T)$. Recall that for $\rho \in \Sigma_{0}(n-k, n), \rho^{*} \in \Sigma_{0}(k-1, n)$ is defined such that $\lambda_{\rho}(\mathrm{d} \lambda)_{\rho^{*}} \in$ $\dot{\mathcal{P}}_{n-k+1} \Lambda^{k}(T)$. In the definition of the isomorphism below we will also use the representation (4.4) of any element $\mathcal{P}_{r}^{-} \Lambda^{k}(T)$.

Theorem 4.22. For $1 \leq k \leq n, r \geq n-k+1$, the map

$$
\sum_{\rho \in \Sigma_{0}(n-k, n)} a_{\rho} \phi_{\rho} \mapsto \sum_{\rho \in \Sigma_{0}(n-k, n)} a_{\rho} \lambda_{\rho}(\mathrm{d} \lambda)_{\rho^{*}},
$$

where $a_{\rho}=a_{\rho}\left(\lambda_{\rho(0)}, \lambda_{\rho(0)+1}, \ldots, \lambda_{n}\right) \in \mathcal{P}_{r+k-n-1}(T)$, defines an isomorphism of $\mathcal{P}_{r+k-n}^{-} \Lambda^{n-k}(T)$ onto $\stackrel{\circ}{\mathcal{P}}_{r} \Lambda^{k}(T)$.

Proof. As Theorem 4.8 implies that $\operatorname{dim} \stackrel{\circ}{\mathcal{P}}_{r} \Lambda^{k}(T) \leq \operatorname{dim} \mathcal{P}_{r+k-n}^{-} \Lambda^{n-k}(T)$, it is enough to show that the map is injective. However, if

$$
\sum_{\rho \in \Sigma_{0}(n-k, n)} a_{\rho} \lambda_{\rho}(\mathrm{d} \lambda)_{\rho^{*}} \equiv 0
$$

and the coefficients $a_{\rho}$ are of the form given above, then we must have

$$
\sum_{\substack{\rho \in \Sigma_{0}(n-k, n) \\ \rho(0)=0}} a_{\rho} \lambda_{\rho}(\mathrm{d} \lambda)_{\rho^{*}} \equiv 0,
$$

since none of the other terms will have $\lambda_{0}$ as factor. Furthermore the set $\left\{(\mathrm{d} \lambda)_{\rho^{*}}\right\}$, where $\rho$ is taken to be in $\Sigma_{0}(n-k, n)$ with $\rho(0)=0$, is a basis for Alt $\mathbb{R}^{n}$. Therefore, all $a_{\rho}$, with $\rho(0)=0$, are identically equal to zero. A simple inductive argument on $\rho(0)$ now implies that all $a_{\rho}$ are identically equal to zero. This completes the proof.

If we apply this result to a subsimplex $f$ instead of $T$, we immediately obtain the following.

Corollary 4.23. Let $f=f_{\sigma} \in \Delta(T)$ with $k \leq \operatorname{dim} f \leq r+k-1$. Then the map

$$
\sum_{\rho \in \Sigma_{0}(\operatorname{dim} f-k, \operatorname{dim} f)} a_{\rho} \phi_{\sigma \circ \rho} \mapsto \sum_{\rho \in \Sigma_{0}(\operatorname{dim} f-k, \operatorname{dim} f)} a_{\rho} \lambda_{\sigma \circ \rho}(\mathrm{d} \lambda)_{\sigma \circ \rho^{*}},
$$


where $a_{\rho}=a_{\rho}\left(\lambda_{\sigma \circ \rho(0)}, \lambda_{\sigma(\rho(0)+1)}, \ldots, \lambda_{\sigma(\operatorname{dim} f)}\right) \in \mathcal{P}_{r+k-\operatorname{dim} f-1}(f)$, defines an isomorphism of $\mathcal{P}_{r+k-\operatorname{dim} f}^{-} \Lambda^{\operatorname{dim} f-k}(f)$ onto $\stackrel{\circ}{\mathcal{P}}_{r} \Lambda^{k}(f)$.

From the unique representation

$$
\sum_{\rho \in \Sigma_{0}(\operatorname{dim} f-k, \operatorname{dim} f)} a_{\rho} \lambda_{\sigma \circ \rho}(\mathrm{d} \lambda)_{\sigma \circ \rho^{*}},
$$

where $a_{\rho}=a_{\rho}\left(\lambda_{\sigma \circ \rho(0)}, \lambda_{\sigma(\rho(0)+1)}, \ldots, \lambda_{\sigma(\operatorname{dim} f)}\right) \in \mathcal{P}_{r+k-\operatorname{dim} f-1}(f)$, we obtain an extension operator

$$
E_{f, T}^{k}: \stackrel{\circ}{\mathcal{P}}_{r} \Lambda^{k}(f) \rightarrow \mathcal{P}_{r} \Lambda^{k}(T),
$$

which is analogous, but not identical, to the extension operator introduced in Section 4.7. Furthermore, as above we can show that if $f, g \in \Delta(T)$, with dimensions at least $k$ and $f \nsubseteq g$, then

$$
\operatorname{Tr}_{g}\left(E_{f, T}^{k} \omega\right)=0, \quad \omega \in \stackrel{\circ}{\mathcal{P}}_{r} \Lambda^{k}(f) .
$$

We can therefore again conclude that the sum of

$$
V(f)=E_{f, T}^{k} \stackrel{\circ}{\mathcal{P}}_{r} \Lambda^{k}(f), \quad f \in \Delta(T), \operatorname{dim} f \geq k
$$

is direct. Finally, it follows from Theorem 4.10 that

$$
\operatorname{dim} \mathcal{P}_{r} \Lambda^{k}(T)=\sum_{\substack{f \in \Delta(T) \\ \operatorname{dim} f \geq k}} \operatorname{dim} V(f)
$$

Hence, Theorem 4.21 is established.

\subsection{The canonical projection operators}

In this subsection, we shall define projection operators $\Pi$ from the spaces $C^{0} \Lambda^{k}(T)$ of continuous differential forms on $T$ onto the spaces $\mathcal{P}_{r} \Lambda^{k}(T)$ and $\mathcal{P}_{r}^{-} \Lambda^{k}(T)$, and show the commutativity property $\mathrm{d} \Pi=\Pi \mathrm{d}$. By Theorems 4.8 and 4.12 , for $0 \leq k \leq n, r \geq 1, \omega \in \mathcal{P}_{r} \Lambda^{k}(T)$ is uniquely determined by the quantities

$$
\int_{f} \operatorname{Tr}_{f} \omega \wedge \eta, \quad \eta \in \mathcal{P}_{r+k-\operatorname{dim} f}^{-} \Lambda^{\operatorname{dim} f-k}(f), \quad f \in \Delta(T),
$$

and $\omega \in \mathcal{P}_{r}^{-} \Lambda^{k}(T)$ is uniquely determined by the quantities

$$
\int_{f} \operatorname{Tr}_{f} \omega \wedge \eta, \quad \eta \in \mathcal{P}_{r+k-\operatorname{dim} f-1} \Lambda^{\operatorname{dim} f-k}(f), \quad f \in \Delta(T) .
$$

Hence, for $\omega \in \Lambda^{k}(T)$, we define the projection operators $\Pi_{r}^{k}$ mapping $\Lambda^{k}(T)$ to $\mathcal{P}_{r} \Lambda^{k}(T)$ and $\Pi_{r-}^{k}$ mapping $\Lambda^{k}(T)$ to $\mathcal{P}_{r}^{-} \Lambda^{k}(T)$ by the relations

$$
\int_{f} \operatorname{Tr}_{f}\left(\omega-\Pi_{r}^{k} \omega\right) \wedge \eta=0, \quad \eta \in \mathcal{P}_{r+k-\operatorname{dim} f}^{-} \Lambda^{\operatorname{dim} f-k}(f), \quad f \in \Delta(T),
$$


and

$$
\int_{f} \operatorname{Tr}_{f}\left(\omega-\Pi_{r-}^{k} \omega\right) \wedge \eta=0, \quad \eta \in \mathcal{P}_{r+k-\operatorname{dim} f-1} \Lambda^{\operatorname{dim} f-k}(f), \quad f \in \Delta(T) .
$$

An easy consequence of the definitions is that the projection operators commute with affine transformations. That is, if $\Phi: T^{\prime} \rightarrow T$ is an affine map, then the following diagrams commute:

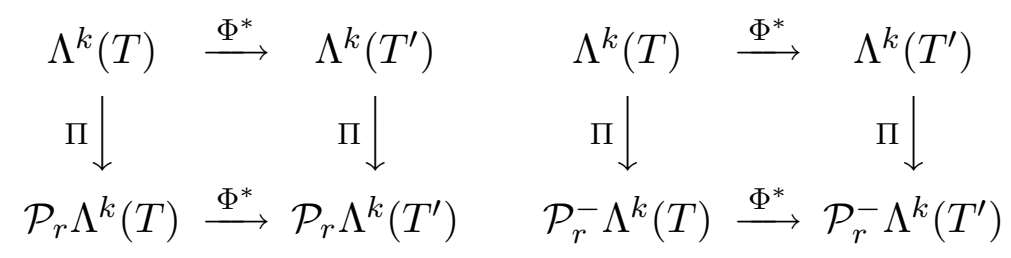

This follows from the facts that the pullback by an affine isomorphism respects the trace operation, wedge product, and integral, and that the $\mathcal{P}_{r} \Lambda^{k}$ and $\mathcal{P}_{r}^{-} \Lambda^{k}$ spaces are affine-invariant.

We now show that these projection operators commute with $\mathrm{d}$. More specifically, we establish the following lemma.

Lemma 4.24. The following four diagrams commute:

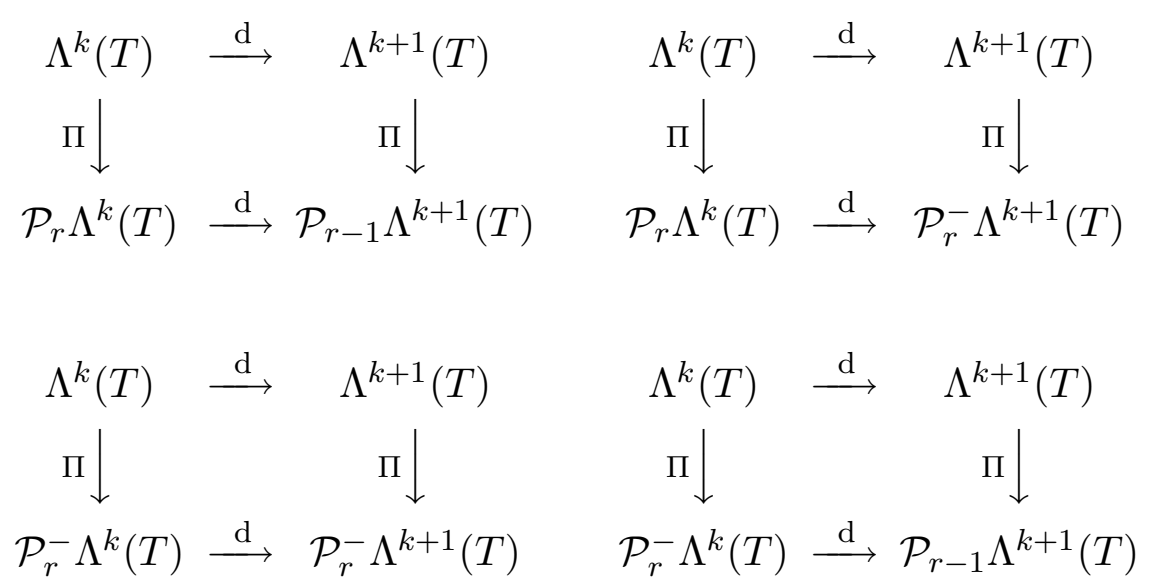

Proof. Let $\Pi_{r}^{k}$ denote the projection onto $\mathcal{P}_{r} \Lambda^{k}(T)$. Then the first diagram asserts that $\mu:=\mathrm{d} \Pi_{r}^{k} \omega-\Pi_{r-1}^{k+1} \mathrm{~d} \omega=0$. From Lemma 3.8, we know that $\mathrm{d} \Pi_{r}^{k} \omega-\Pi_{r-1}^{k+1} \mathrm{~d} \omega \in \mathcal{P}_{r-1} \Lambda^{k+1}(T)$. Thus $\mu=0$ if

$$
\int_{f} \operatorname{Tr}_{f} \mu \wedge \eta=0, \quad \eta \in \mathcal{P}_{r+k-\operatorname{dim} f}^{-} \Lambda^{\operatorname{dim} f-k-1}(f), \quad f \in \Delta(T) .
$$

But, by Stokes' theorem and the fact that $\operatorname{Tr}_{f} \mathrm{~d}=\mathrm{d}_{f} \operatorname{Tr}_{f}$, we get

$$
\begin{aligned}
\int_{f} \operatorname{Tr}_{f} \mathrm{~d} \Pi_{r}^{k} \omega \wedge \eta & =\int_{f} \mathrm{~d}_{f} \operatorname{Tr}_{f} \Pi_{r}^{k} \omega \wedge \eta \\
& =(-1)^{k-1} \int_{f} \operatorname{Tr}_{f} \Pi_{r}^{k} \omega \wedge \mathrm{d}_{f} \eta+\int_{\partial f} \operatorname{Tr} \Pi_{r}^{k} \omega \wedge \operatorname{Tr} \eta
\end{aligned}
$$




$$
\begin{aligned}
& =(-1)^{k-1} \int_{f} \operatorname{Tr}_{f} \omega \wedge \mathrm{d}_{f} \eta+\int_{\partial f} \operatorname{Tr} \omega \wedge \operatorname{Tr} \eta \\
& =\int_{f} \mathrm{~d}_{f} \operatorname{Tr}_{f} \omega \wedge \eta=\int_{f} \operatorname{Tr}_{f} \Pi_{r-1}^{k+1} \mathrm{~d} \omega \wedge \eta .
\end{aligned}
$$

A similar argument is used to establish the other three identities.

\section{Finite element differential forms and their cohomology}

Throughout this section, we assume that $\Omega$ is a bounded polyhedral domain in $\mathbb{R}^{n}$ which is partitioned into a finite set of $n$-simplices $\mathcal{T}$. These $n$ simplices determine a simplicial decomposition of $\Omega$. That is, their union is the closure of $\Omega$, and the intersection of any two is either empty or a common subsimplex of each. Adopting the terminology of the two-dimensional case, we will refer to $\mathcal{T}$ as a triangulation of $\Omega$. In this section we will define two families of spaces of finite element differential forms with respect to the triangulation $\mathcal{T}$, denoted $\mathcal{P}_{r} \Lambda^{k}(\mathcal{T})$ and $\mathcal{P}_{r}^{-} \Lambda^{k}(\mathcal{T})$, which are subspaces of the corresponding Sobolev space $H \Lambda^{k}(\Omega)$. In particular, we will show how these finite element spaces lead to a number of finite element subcomplexes of the de Rham complex.

\subsection{Finite element differential forms}

\section{Interelement continuity}

Recall that $\Delta_{j}(T)$ denotes the set of all $j$-dimensional subsimplices of the simplex $T$. Furthermore, we let $\Delta_{j}(\mathcal{T})$ be the set of all $j$-dimensional subsimplices generated by $\mathcal{T}$, i.e.,

$$
\Delta_{j}(\mathcal{T})=\bigcup_{T \in \mathcal{T}} \Delta_{j}(T)
$$

It follows from Stokes' theorem that if $\omega \in L^{2} \Lambda^{k}(\Omega)$ is piecewise smooth with respect to the triangulation $\mathcal{T}$, then $\omega \in H \Lambda^{k}(\Omega)$ if and only if $\operatorname{Tr} \omega$ is single-valued at all $f \in \Delta_{n-1}(\mathcal{T})$. In other words, if $T_{1}, T_{2} \in \mathcal{T}$ have the common face $f \in \Delta_{n-1}(\mathcal{T})$, then

$$
\operatorname{Tr}_{T_{1}, f} \omega=\operatorname{Tr}_{T_{2}, f} \omega
$$

where $\operatorname{Tr}_{T, f}$ denotes the trace on $f$ derived from $\left.\omega\right|_{T}$. However, if $\operatorname{Tr}_{T_{1}, f} \omega=$ $\operatorname{Tr}_{T_{2}, f} \omega$, then clearly $\operatorname{Tr}_{T_{1}, g} \omega=\operatorname{Tr}_{T_{2}, g} \omega$ for all $g \in \Delta_{j}(f), k \leq j \leq n-1$. Hence, we have the following characterization of piecewise smooth functions in $H \Lambda^{k}(\Omega)$.

Lemma 5.1. Let $\omega \in L^{2} \Lambda^{k}(\Omega)$ be piecewise smooth with respect to the triangulation $\mathcal{T}$. The following statements are equivalent:

(1) $\omega \in H \Lambda^{k}(\Omega)$, 
(2) $\operatorname{Tr} \omega$ is single-valued for all $f \in \Delta_{n-1}(\mathcal{T})$,

(3) $\operatorname{Tr} \omega$ is single-valued for all $f \in \Delta_{j}(\mathcal{T}), k \leq j \leq n-1$.

Shape functions and degrees of freedom

For $r \geq 1$, the spaces $\mathcal{P}_{r} \Lambda^{k}(\mathcal{T})$ and $\mathcal{P}_{r}^{-} \Lambda^{k}(\mathcal{T})$ are defined using the polynomial spaces $\mathcal{P}_{r} \Lambda^{k}(T)$ and $\mathcal{P}_{r}^{-} \Lambda^{k}(T)$, respectively, as shape functions, and enforcing just enough interelement continuity to ensure inclusion in $H \Lambda^{k}(\Omega)$. That is, we define

$$
\begin{aligned}
\mathcal{P}_{r} \Lambda^{k}(\mathcal{T}) & =\left\{\omega \in H \Lambda^{k}(\Omega)|\omega|_{T} \in \mathcal{P}_{r} \Lambda^{k}, T \in \mathcal{T}\right\}, \\
\mathcal{P}_{r}^{-} \Lambda^{k}(\mathcal{T}) & =\left\{\omega \in H \Lambda^{k}(\Omega)|\omega|_{T} \in \mathcal{P}_{r}^{-} \Lambda^{k}, T \in \mathcal{T}\right\} .
\end{aligned}
$$

Hence, all elements of these spaces have to satisfy the continuity requirements specified in Lemma 5.1.

Degrees of freedom for these spaces are easily determined from the case of a single simplex treated in Section 4 . For the space $\mathcal{P}_{r} \Lambda^{k}(\mathcal{T})$ we use

$$
\int_{f} \operatorname{Tr}_{f} \omega \wedge \nu, \quad \nu \in \mathcal{P}_{r-j+k}^{-} \Lambda^{j-k}(f), \quad f \in \Delta_{j}(\mathcal{T}),
$$

for $k \leq j \leq \min (n, r+k-1)$. By Lemma 5.1, the quantities in (5.1) are well-defined for $\omega \in \mathcal{P}_{r} \Lambda^{k}\left(\mathcal{T}_{h}\right)$. In view of Section 4.8, the quantities corresponding to $f \subset T$ determine $\left.\omega\right|_{T} \in \mathcal{P}_{r} \Lambda^{k}(T)$. Therefore, if we choose any basis for the test spaces $\mathcal{P}_{r-j+k}^{-} \Lambda^{j-k}(f)$ and assign arbitrary values to $\int_{f} \operatorname{Tr}_{f} \omega \wedge \nu$ for basis elements $\nu$ and for all $f \in \Delta_{j}(\mathcal{T})$, we determine a unique piecewise polynomial $\omega$. Moreover, if $F \in \Delta_{n-1}(T)$, then $\operatorname{Tr}_{F} \omega \in \mathcal{P}_{r} \Lambda^{k}(F)$ and so is determined by the quantities in (5.1) corresponding to $f \subset F$. Thus $\operatorname{Tr}_{F} \omega$ is single-valued and so $\omega \in H \Lambda^{k}(\Omega)$. This establishes that we have a set of degrees of freedom $\mathcal{P}_{r} \Lambda^{k}(\mathcal{T}$ ) (and so this is truly a finite element space).

Analogously, the degrees of freedom for the space $\mathcal{P}_{r}^{-} \Lambda^{k}(\mathcal{T})$ are given by

$$
\int_{f} \operatorname{Tr}_{f} \omega \wedge \nu, \quad \nu \in \mathcal{P}_{r-j+k-1} \Lambda^{j-k}(f), \quad f \in \Delta_{j}(\mathcal{T}),
$$

for $k \leq j \leq \min (n, r+k-1)$.

In 2 and 3 dimensions we may use proxy fields to identify these spaces of finite element differential forms with finite element spaces of scalar and vector functions. In the tables below, we summarize the correspondences between spaces of finite element differential forms and classical finite element spaces: the Lagrange elements (Ciarlet 1978), the Raviart-Thomas introduced in two dimensions by Raviart and Thomas (1977) and generalized to three dimensions by Nédélec (1980); the Brezzi-Douglas-Marini elements introduced by Brezzi, Douglas and Marini (1985) and generalized to three dimensions by Nédélec (1986) and Brezzi, Douglas, Durán and 
Table 5.1. Correspondences between finite element differential forms and the classical finite element spaces for $n=2$.

\begin{tabular}{c|cl}
\hline \hline$k$ & $\Lambda_{h}^{k}(\Omega)$ & \multicolumn{1}{c}{ Classical finite element space } \\
\hline 0 & $\mathcal{P}_{r} \Lambda^{0}(\mathcal{T})$ & Lagrange elements of degree $\leq r$ \\
1 & $\mathcal{P}_{r} \Lambda^{1}(\mathcal{T})$ & Brezzi-Douglas-Marini $H($ div) elements of degree $\leq r$ \\
2 & $\mathcal{P}_{r} \Lambda^{2}(\mathcal{T})$ & discontinuous elements of degree $\leq r$ \\
\hline 0 & $\mathcal{P}_{r}^{-} \Lambda^{0}(\mathcal{T})$ & Lagrange elements of degree $\leq r$ \\
1 & $\mathcal{P}_{r}^{-} \Lambda^{1}(\mathcal{T})$ & Raviart-Thomas $H($ div $)$ elements of order $r-1$ \\
2 & $\mathcal{P}_{r}^{-} \Lambda^{2}(\mathcal{T})$ & discontinuous elements of degree $\leq r-1$ \\
\hline
\end{tabular}

Table 5.2. Correspondences between finite element differential forms and the classical finite element spaces for $n=3$.

\begin{tabular}{c|cl}
\hline \hline$k$ & $\Lambda_{h}^{k}(\Omega)$ & \multicolumn{1}{c}{ Classical finite element space } \\
\hline 0 & $\mathcal{P}_{r} \Lambda^{0}(\mathcal{T})$ & Lagrange elements of degree $\leq r$ \\
1 & $\mathcal{P}_{r} \Lambda^{1}(\mathcal{T})$ & Nédélec 2nd-kind $H($ curl) elements of degree $\leq r$ \\
2 & $\mathcal{P}_{r} \Lambda^{2}(\mathcal{T})$ & Nédélec 2nd-kind $H($ div $)$ elements of degree $\leq r$ \\
3 & $\mathcal{P}_{r} \Lambda^{3}(\mathcal{T})$ & discontinuous elements of degree $\leq r$ \\
\hline 0 & $\mathcal{P}_{r}^{-} \Lambda^{0}(\mathcal{T})$ & Lagrange elements of degree $\leq r$ \\
1 & $\mathcal{P}_{r}^{-} \Lambda^{1}(\mathcal{T})$ & Nédélec 1st-kind $H($ curl) elements of order $r-1$ \\
2 & $\mathcal{P}_{r}^{-} \Lambda^{2}(\mathcal{T})$ & Nédélec 1st-kind $H($ div $)$ elements of order $r-1$ \\
3 & $\mathcal{P}_{r}^{-} \Lambda^{3}(\mathcal{T})$ & discontinuous elements of degree $\leq r-1$ \\
\hline
\end{tabular}

Fortin (1987); and the spaces of discontinuous elements of degree r, i.e., all piecewise polynomials of degree no greater than $r$.

\subsection{The canonical projections}

In Section 4.9, we defined for each simplex $T$ a projection $\Pi_{T}: C^{0} \Lambda^{k}(T) \rightarrow$ $\mathcal{P}_{r} \Lambda^{k}(T)$. We can then define $\Pi=\Pi_{\mathcal{T}}: C^{0} \Lambda^{k}(\Omega) \rightarrow \mathcal{P}_{r} \Lambda^{k}(\mathcal{T})$ by

$$
\left.\left(\Pi_{\mathcal{T}} \omega\right)\right|_{T}=\Pi_{T}\left(\left.\omega\right|_{T}\right)
$$

(Note that the degrees of freedom used to determine $\Pi_{T}$ ensure that the traces of $\Pi_{\mathcal{T}} \omega$ on faces are single-valued.) Equivalently, $\Pi_{\mathcal{T}} \omega$ is determined 
by the equations

$$
\int_{f} \operatorname{Tr}_{f} \Pi_{\mathcal{T}} \omega \wedge \nu=\int_{f} \operatorname{Tr}_{f} \omega \wedge \nu, \quad \nu \in \mathcal{P}_{r-j+k}^{-} \Lambda^{j-k}(f), \quad f \in \Delta_{j}(\mathcal{T}),
$$

for $k \leq j \leq \min (n, r+k-1)$. Of course, an analogous operator $\Pi=$ $\Pi_{\mathcal{T}}: C^{0} \Lambda^{k}(\Omega) \rightarrow \mathcal{P}_{r}^{-} \Lambda^{k}(\mathcal{T})$ is defined similarly. From Lemma 4.24, we get commutativity of the projections with the exterior derivative.

Theorem 5.2. The following four diagrams commute:

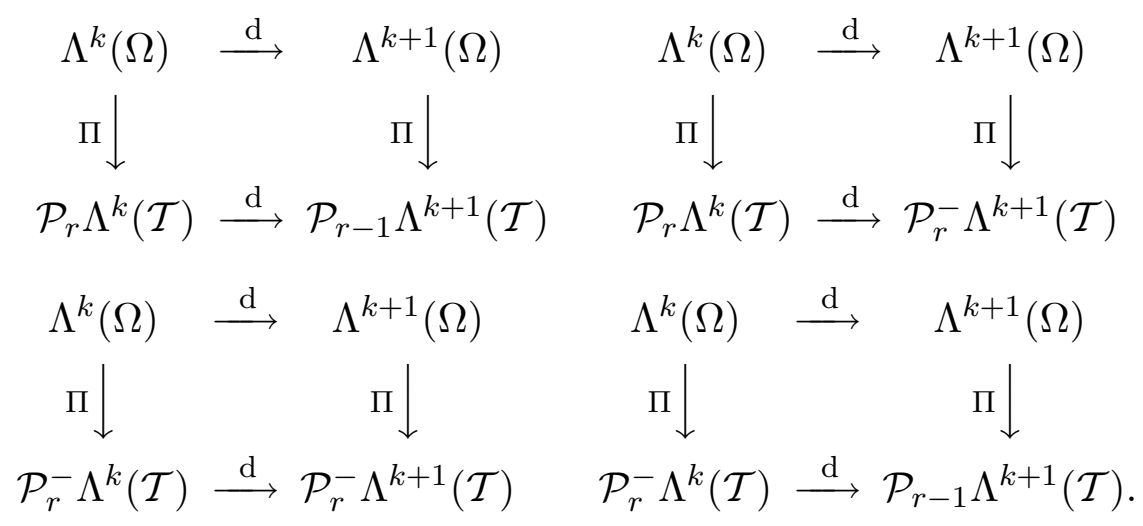

\subsection{Error estimates}

Now we consider error bounds for $\|\omega-\Pi \omega\|$. To this end we need to consider not just a single triangulation $\mathcal{T}$ but a family of triangulations $\mathcal{T}_{h}$ of $\Omega$ indexed by the discretization parameter

$$
h=\max _{T \in \mathcal{T}_{h}} \operatorname{diam} T .
$$

We assume that the discretization parameter runs over a set of positive values bounded by some $h_{\max }$ and accumulating at zero.

In order to obtain error estimates we will assume that there exists a constant $C_{\text {mesh }}>0$, called the mesh regularity constant, for which

$$
|h|^{n} \leq C_{\text {mesh }}|T|, \quad T \in \mathcal{T}_{h}
$$

where $|T|$ denotes the volume of the simplex $T$. This assumption has two consequences. First, it enforces the shape regularity of the triangulation family, meaning that each simplex is Lipschitz diffeomorphic to a ball with uniform bounds on the Lipschitz constants of the diffeormorphism and its inverse. Second it implies that the family $\left\{\mathcal{T}_{h}\right\}$ is quasiuniform in the sense that ratio of diameters of elements in $\mathcal{T}_{h}$ are bounded uniformly in $h$. The quasiuniformity property is too restrictive for many applications, and often it can be avoided by more sophisticated analysis, using, instead of (5.3), the weaker assumption $|\operatorname{diam} T|^{n} \leq C|T|$. However, here we shall assume quasiuniformity in order to avoid some technical difficulties, and so use (5.3). 
We now state bounds for the error in the canonical projection of a differential form, in terms of the $W_{p}^{s} \Lambda^{k}(\Omega)$ seminorm of the form and a power of the mesh parameter $h$.

Theorem 5.3. Denote by $\Pi_{h}$ the canonical projection of $\Lambda^{k}(\Omega)$ onto either $\mathcal{P}_{r} \Lambda^{k}\left(\mathcal{T}_{h}\right)$ or $\mathcal{P}_{r+1}^{-} \Lambda^{k}\left(\mathcal{T}_{h}\right)$. Let $1 \leq p \leq \infty$ and $(n-k) / p<s \leq r+1$. Then $\Pi_{h}$ extends boundedly to $W_{p}^{s} \Lambda^{k}(\Omega)$, and there exists a constant $c$ independent of $h$, such that

$$
\left\|\omega-\Pi_{h} \omega\right\|_{L^{p} \Lambda^{k}(\Omega)} \leq C h^{s}|\omega|_{W_{p}^{s} \Lambda^{k}(\Omega)}, \quad \omega \in W_{p}^{s} \Lambda^{k}(\Omega) .
$$

Proof. We shall show the result element by element. That is, we shall show that $\Pi_{T}$ extends boundedly to $W_{p}^{s} \Lambda^{k}(T)$ and

$$
\left\|\omega-\Pi_{T} \omega\right\|_{L^{p} \Lambda^{k}(T)} \leq C h^{s}|\omega|_{W_{p}^{s} \Lambda^{k}(T)}, \quad \omega \in \Lambda^{k}(T),
$$

for each element $T \in \mathcal{T}_{h}$, with the constant independent of $T$ and $h$.

Since $s>(n-k) / p$, the Sobolev embedding theorem implies that $\omega \in$ $W_{p}^{s}(T)$ admits traces on subsimplices of dimension $k$ and so the operator $\Pi_{T}$ is bounded on $W_{p}^{s}(T)$ with the bound

$$
\left\|\left(I-\Pi_{T}\right) \omega\right\|_{L^{p} \Lambda^{k}(T)} \leq c\|\omega\|_{W_{p}^{s} \Lambda^{k}(T)},
$$

for some constant $c$ (which may depend on $T$ ). But $I-\Pi_{T}$ annihilates polynomial forms of degree $r$ or less, and $s \leq r+1$, and so the BrambleHilbert lemma (Brenner and Scott 2002, Lemma 4.3.8) implies that

$$
\left\|\left(I-\Pi_{T}\right) \omega\right\|_{L^{p} \Lambda^{k}(T)} \leq c(T)|\omega|_{W_{p}^{s} \Lambda^{k}(T)},
$$

where now the Sobolev seminorm appears on the right-hand side. We choose $c(T)$ to be the least constant $c$ for which (5.5) holds. Then $c(T)$ is a continuous function of $T$, or, more precisely, of its vertices, which belong to the open set

$$
\left\{\left(x_{0}, \ldots, x_{n}\right) \in\left(\mathbb{R}^{n}\right)^{n+1} \mid \operatorname{det}\left[x_{1}-x_{0}, \ldots, x_{n}-x_{0}\right] \neq 0\right\} .
$$

To get from (5.5) to (5.4) we use scaling. Let $x_{0}$ denote the first vertex of $T$, and define $\Phi(x)=\left(x-x_{0}\right) / h$, an invertible affine map depending on $T$ and $h$ which maps $T$ to $\hat{T}:=\Phi(T)$. See Figure 5.1. For $\omega \in W_{p}^{s} \Lambda^{k}(T)$, define $\hat{\omega}=\Phi^{-1 *} \omega \in W_{p}^{s} \Lambda^{k}(\hat{T})$. It is easy to check that $\Pi_{\hat{T}} \hat{\omega}=\Phi^{-1 *}\left(\Pi_{T} \omega\right)$ and so that

$$
\begin{gathered}
\left\|\left(I-\Pi_{\hat{T}}\right) \hat{\omega}\right\|_{L^{p} \Lambda^{k}(\hat{T})}=h^{k-n / p}\left\|\left(I-\Pi_{T}\right) \omega\right\|_{L^{p} \Lambda^{k}(T)} \\
|\hat{\omega}|_{W_{p}^{s} \Lambda^{k}(\hat{T})}=h^{s+k-n / p}|\omega|_{W_{p}^{s} \Lambda^{k}(T)} .
\end{gathered}
$$




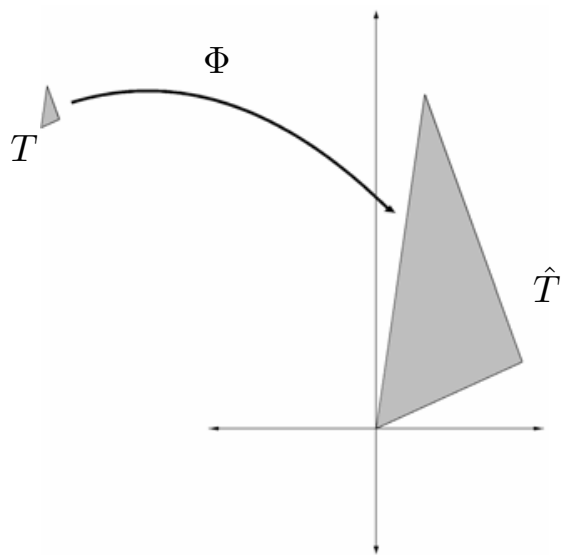

Figure 5.1. Scaling of a simplex by dilation and translation.

Therefore

$$
\begin{aligned}
\left\|\left(I-\Pi_{T}\right) \omega\right\|_{L^{p} \Lambda^{k}(T)} & =h^{n / p-k}\left\|\left(I-\Pi_{\hat{T}}\right) \hat{\omega}\right\|_{L^{p} \Lambda^{k}(\hat{T})} \\
& \leq c(\hat{T}) h^{n / p-k}|\hat{\omega}|_{W_{p}^{s} \Lambda^{k}(\hat{T})} \\
& =c(\hat{T}) h^{s}\left\|\left(I-\Pi_{T}\right) \omega\right\|_{L^{p} \Lambda^{k}(T)}
\end{aligned}
$$

whence

$$
c(T)=c(\hat{T}) h^{s}
$$

But the mesh regularity assumption implies that there exists positive constants $K_{1}$ and $K_{2}$ depending only on $C_{\text {mesh }}$ such that

$$
\operatorname{diam} \hat{T} \leq K_{1}, \quad|\hat{T}| \geq K_{2} .
$$

Together with the fact that $\hat{T}$ has a vertex at the origin, these conditions confine the vertices to a compact set, and so there is a constant $C$, independent of $T \in \mathcal{T}_{h}$ and $h$ so that $c(\hat{T}) \leq C$. Combining with (5.5) and (5.6), we obtain (5.4) and hence the theorem.

Remark. This proof uses the standard elements of localization, Sobolev embedding, the Bramble-Hilbert lemma, and scaling, but is a little unusual in that we scale by dilation and translation only, and thus we get a family of scaled elements $\hat{T}$, which is, in an appropriate sense, compact. The more standard proof uses affine scaling to a fixed reference element and so avoids the compactness argument. We have chosen to use dilation and compactness here, because we will use the same technique again when bounding smoothed projections.

\subsection{Projections and smoothing}

The estimates just obtained for the canonical projections are not sufficient for our needs. For example, they do not furnish bounds for the projection 
into $\mathcal{P}_{r} \Lambda^{1}\left(\mathcal{T}_{h}\right)$ in terms of the $H^{1}$-norm in three dimensions $(s=1, p=2$, $k=1, n=3$ is not allowed). They provide no estimate in terms of any $p=2$ based Sobolev norm for the projection into $\mathcal{P}_{1} \Lambda^{0}\left(\mathcal{T}_{h}\right)$ (i.e., the nodal interpolant) in more than three dimensions.

The difficulty with the canonical projection is that, due to its dependence on traces on subsimplices, it is not bounded on spaces with insufficient smoothness. This same problem shows up in that it is not easy to use the canonical projections in a cochain projection from a version of the de Rham sequence onto a discrete subcomplex. If the spaces in the de Rham sequence are smooth enough that the projection operators are bounded on them, they are generally too smooth to include the finite element spaces.

In finite element theory the Clément interpolant (Clément 1975) is often invoked to overcome problems of this sort. In our situation, the Clément interpolant would be defined by assigning to a given form in $\omega \in L^{2} \Lambda^{k}(\Omega)$ a finite element differential form $\check{\Pi}_{h} \omega$ specified by the degrees of freedom in the finite element space. For a degree of freedom associated with a subsimplex $f$, the Clément interpolant takes the value of this degree of freedom not from $\omega$, but rather from the $L^{2}$-projection of $\omega$ into the space of polynomials forms of degree $r$ on the union of the simplices in $\mathcal{T}_{h}$ containing $f$. This construction yields an operator which is bounded on $L^{2}$ and satisfies optimal order error bounds:

$$
\left\|\omega-\check{\Pi}_{h} \omega\right\|_{L^{p} \Lambda^{k}(\Omega)} \leq c h^{s}\|\omega\|_{W_{p}^{s} \Lambda^{k}(\Omega)}, \quad 0 \leq s \leq r+1 .
$$

However $\check{\Pi}_{h}$ is not a projection (it does not leave the finite element subspace fixed), and it does not commute with the exterior derivative. And so it, too, is not sufficient for our purposes.

To overcome these difficulties, in this subsection we will construct an alternative set of projection operators $\tilde{\Pi}_{h}$ which are bounded from $L^{2} \Lambda^{k}(\Omega)$ to the finite element space. The approach we take is highly influenced by recent work by Schöberl (2005) and Christiansen (2005), partially unpublished. Briefly, to project a form $\omega$ we will first extend it to a slightly larger domain and then regularize. Regularization commutes with exterior differentiation, and the extension is chosen so that it commutes with exterior differentiation as well. Next we use the canonical projection to project the regularized form into the subspace. This procedure gives an operator which is bounded and $L^{2}$ and which commutes with the exterior derivative, but which is not a projection. We remedy this by multiplying by the inverse of the operator restricted to the subspace (which can be shown to exist). The resulting operator still commutes with exterior differentiation, is still bounded on $L^{2}$, and is a projection. From the last two properties we easily obtain optimal error estimates. 
The extension operator

Since we will make use of a smoothing operator defined by convolution with a mollifier function, we will need to extend functions in $H \Lambda^{k}(\Omega)$ to a fixed larger domain $\tilde{\Omega}$ where the closure of $\Omega, \bar{\Omega}$ is contained in $\tilde{\Omega}$. Let the extended domain be of the form $\tilde{\Omega}=\bar{\Omega} \cup \Omega_{o}$, where $\bar{\Omega} \cap \Omega_{o}=\emptyset$. Following Schöberl (2005), we utilize the compactness of the boundary $\partial \Omega$ to construct the outer neighbourhood $\Omega_{o}$, a corresponding interior neighbourhood of $\partial \Omega$, $\Omega_{i} \subset \Omega$, and a Lipschitz continuous bijection $\Psi: \Omega_{o} \cup \partial \Omega \rightarrow \Omega_{i} \cup \partial \Omega$, with the additional properties that $\Psi(x)=x$ on $\partial \Omega$.

Using the mapping $\Psi$, we define an extension operator $E: H \Lambda^{k}(\Omega) \rightarrow$ $H \Lambda^{k}(\tilde{\Omega})$ by

$$
(E \omega)_{x}=\left(\Psi^{*} \omega\right)_{x}, \quad x \in \Omega_{o}
$$

This operator clearly maps $L^{2} \Lambda^{k}(\Omega)$ boundedly into $L^{2} \Lambda^{k}(\tilde{\Omega})$, and since $\mathrm{d} \circ \Psi^{*}=\Psi^{*} \circ \mathrm{d}$, we obtain that $E \in \mathcal{L}\left(H \Lambda^{k}(\Omega), H \Lambda^{k}(\tilde{\Omega})\right)$.

The smoothing operator

As in Christiansen (2005), we will perform a construction where we combine the canonical projections introduced above with a standard smoothing operator defined by convolution with an approximate Dirac delta function. For parameters $\epsilon>0$, we will employ smoothing operators $R_{\epsilon}: L^{2} \Lambda^{k}(\Omega) \rightarrow$ $\Lambda^{k}(\Omega)$ of the form

$$
R_{\epsilon} \omega=\left.\left(\rho_{\epsilon} * E \omega\right)\right|_{\Omega} .
$$

Here $*$ denotes the convolution product, and the mollifier function $\rho_{\epsilon}$ is of the form $\rho_{\epsilon}(x)=\epsilon^{-n} \rho(x / \epsilon)$, where $\rho: \mathbb{R}^{n} \rightarrow \mathbb{R}$ is $C^{\infty}$, is nonnegative with compact support, and with integral equal to 1 . Note that for $\epsilon$ sufficiently small, the operator $R_{\epsilon}: L^{2} \Lambda^{k}(\Omega) \rightarrow \Lambda^{k}(\Omega)$ is well defined, and we have the commutativity property $\mathrm{d} R_{\epsilon}=R_{\epsilon} \mathrm{d}$.

The smoothing parameter has to be related to the triangulation $\mathcal{T}_{h}$. In fact, for more general triangulations than we allow here, it seems that an $x$ dependent smoothing parameter is required. We refer to Christiansen (2005) for such a discussion. However, since we assume that the triangulation is quasiuniform (see (5.3)), we can choose the smoothing parameter proportional to the mesh parameter $h$. Hence, our construction will use the smoothing operator $R_{\epsilon h}$, where $\epsilon>0$ will be chosen independently of the triangulation $\mathcal{T}_{h}$. We will take $\epsilon$ sufficiently small so that

- For $x \in \bar{\Omega}$, the ball of radius $\epsilon h_{\max }$ about $x$ is contained in $\Omega \cup \Omega_{0}$.

- For each $h$, if $x \in T$ for some $T \in \mathcal{T}_{h}$, then the ball of radius $\epsilon h$ about $x$ is contained in the union of the simplices in $\mathcal{T}_{h}$ which intersect $T$.

Uniform bounds

Now we let $\Lambda_{h}^{k}$ denote either $\mathcal{P}_{r} \Lambda^{k}\left(\mathcal{T}_{h}\right)$ or $\mathcal{P}_{r}^{-} \Lambda^{k}\left(\mathcal{T}_{h}\right)$ and denote by $\Pi_{h}$ the canonical interpolation operator onto $\Lambda_{h}^{k}$. 
Since we have restricted to $\epsilon$ sufficiently small, $R_{\epsilon h}$ maps $L^{2} \Lambda^{k}(\Omega)$ into $C \Lambda^{k}(\Omega)$ for all $h$. Then the map $\Pi_{h} R_{\epsilon h}: L^{2} \Lambda^{k}(\Omega) \rightarrow \Lambda_{h}^{k}$ is certainly bounded. The following lemma, we shall prove below via a scaling argument, states that the bound is uniform in $h$ (but not in $\epsilon$ ).

Lemma 5.4. For $\epsilon$ sufficiently small as above, there exists a constant $c(\epsilon)$, such that, the operator norm

$$
\left\|\Pi_{h} R_{\epsilon h}\right\|_{\mathcal{L}\left(L^{2} \Lambda^{k}(\Omega), L^{2} \Lambda^{k}(\Omega)\right)} \leq c(\epsilon),
$$

for all $h$.

The restriction $\left.\Pi_{h} R_{\epsilon h}\right|_{\Lambda_{h}^{k}}$ maps $\Lambda_{h}^{k}$ into itself. The following lemma, which we shall also prove below using scaling, states that it converges the identity as $\epsilon \rightarrow 0$, uniformly in $h$. In it we use the notation $\|\cdot\|_{\mathcal{L}\left(\Lambda_{h}^{k}, \Lambda_{h}^{k}\right)}$ to denote the $L^{2}$ operator norm of an operator $\Lambda_{h}^{k} \rightarrow \Lambda_{h}^{k}$.

Lemma 5.5. There exists a constant $c$, independent of $h$ and $\epsilon$, such that

$$
\left\|I-\left.\Pi_{h} R_{\epsilon h}\right|_{\Lambda_{h}^{k}}\right\|_{\mathcal{L}\left(\Lambda_{h}^{k}, \Lambda_{h}^{k}\right)} \leq c \epsilon .
$$

In view of this lemma, we can choose $\epsilon$ sufficiently small that

$$
\left\|I-\left.\Pi_{h} R_{\epsilon h}\right|_{\Lambda_{h}^{k}}\right\|_{\mathcal{L}\left(\Lambda_{h}^{k}, \Lambda_{h}^{k}\right)} \leq 1 / 2
$$

for all $h$. It follows that $\left.\Pi_{h} R_{\epsilon h}\right|_{\Lambda_{h}^{k}}$ is invertible and that its inverse $J_{h}^{\epsilon}$ : $\Lambda_{h}^{k} \rightarrow \Lambda_{h}^{k}$ satisfies

$$
\left\|J_{h}^{\epsilon}\right\|_{\mathcal{L}\left(\Lambda_{h}^{k}, \Lambda_{h}^{k}\right)} \leq 2
$$

The smoothed projections

Combining these results we can easily complete the construction of the smoothed projections. We fix $\epsilon$ sufficiently small as above and also so that (5.7) holds. Then, for this fixed $\epsilon$, we set

$$
\tilde{\Pi}_{h}=J_{h}^{\epsilon} \Pi_{h} R_{\epsilon h} .
$$

This operator has all the properties we need.

Theorem 5.6. The smoothed projection $\tilde{\Pi}_{h}$ is a projection of $L^{2} \Lambda^{k}(\Omega)$ onto $\Lambda_{h}^{k}$ which commutes with the exterior derivative and satisfies

$$
\left\|\omega-\tilde{\Pi}_{h} \omega\right\| \leq c h^{s}\|\omega\|_{s}, \quad \omega \in H^{s} \Lambda^{k}(\Omega), 0 \leq s \leq r+1 .
$$

Moreover, for all $\omega \in L^{2} \Lambda^{k}(\Omega), \tilde{\Pi}_{h} \omega \rightarrow \omega$ in $L^{2}$ as $h \rightarrow 0$.

Proof. By construction $\tilde{\Pi}_{h}$ is a bounded linear operator from $L^{2} \Lambda^{k}(\Omega)$ to $\Lambda_{h}^{k}$ which commutes with $\mathrm{d}$, so we need only establish the error bounds. Using Lemma 5.4 and (5.8), we have that $\tilde{\Pi}_{h}$ is uniformly bounded in 
$\mathcal{L}\left(L^{2} \Lambda^{k}(\Omega), L^{2} \Lambda^{k}(\Omega)\right)$. Thus from the projection property we have

$$
\left\|\omega-\tilde{\Pi}_{h} \omega\right\|=\inf _{\mu \in \Lambda_{h}^{k}}\left\|\left(I-\tilde{\Pi}_{h}\right)(\omega-\mu)\right\| \leq c \inf _{\mu \in \Lambda_{h}^{k}}\|\omega-\mu\| \leq c h^{s}\|\omega\|_{s},
$$

where the last inequality can be proved, e.g., by using the Clément interpolant. The final pointwise estimate follows from the estimate for $s=1$ and the fact that $H^{1} \Lambda^{k}(\Omega)$ is dense in $L^{2} \Lambda^{k}(\Omega)$.

Proofs of the uniform bounds

We now turn to the proofs of Lemmas 5.4 and 5.5. As in the proof of Theorem 5.3 we use localization and scaling and compactness, but the situation is complicated by the fact that the smoothing operator is not entirely local: the restriction of $R_{\epsilon h} \omega$ to a simplex $T$ is not determined by $\left.\omega\right|_{T}$. However, since we have assumed that $\epsilon$ is sufficiently small, $\left.\left(R_{\epsilon h} \omega\right)\right|_{T}$ is determined by $\left.\omega\right|_{T^{\star}}$ where $T^{\star}=\bigcup \mathcal{T}_{h}(T)$ and.

$$
\mathcal{T}_{h}(T):=\left\{T^{\prime} \in \mathcal{T}_{h} \mid T^{\prime} \cap T \neq \emptyset\right\},
$$

is the macroelement in $\mathcal{T}_{h}$ determined by $T$ (see Figure 5.2). We shall write

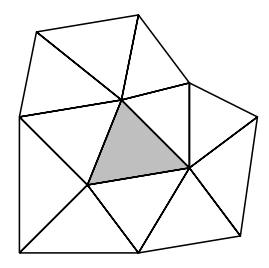

Figure 5.2. Macroelement of an element $T$ : the triangulation is $\mathcal{T}_{h}(T)$, the triangulated region is $T^{\star}$, and the shaded simplex is $T$.

$\Lambda_{h}^{k}(T)$ and $\Lambda_{h}^{k}\left(T^{\star}\right)$ for the space of restrictions of elements of $\Lambda_{h}^{k}$ to $T$ or $T^{\star}$. The former is just the polynomial space $\mathcal{P}_{r} \Lambda^{k}(T)$ or $\mathcal{P}_{r}^{-} \Lambda^{k}(T)$, while the later is equal to either $\mathcal{P}_{r} \Lambda^{k}\left(\mathcal{T}_{h}(T)\right)$ or $\mathcal{P}_{r}^{-} \Lambda^{k}\left(\mathcal{T}_{h}(T)\right.$. Now the shape regularity property implies bounded overlap of the $T^{\star}$, so

$$
\sum_{T \in \mathcal{T}_{h}}\|\omega\|_{H^{s} \Lambda^{k}\left(T^{\star}\right)} \leq c\|\omega\|_{H^{s} \Lambda^{k}(\Omega)} .
$$

Therefore, to prove Lemma 5.4, it suffices to show that

$$
\left\|\Pi_{T} R_{\epsilon h}\right\|_{\mathcal{L}\left(L^{2} \Lambda^{k}\left(T^{\star}\right), L^{2} \Lambda^{k}(T)\right)} \leq c(\epsilon)
$$

with $c(\epsilon)$ uniform over $T \in \mathcal{T}_{h}$ and over $h$. (There are some small modifications needed for the simplexes $T$ intersecting $\partial \Omega$.) Similarly, for Lemmas 5.5 it is sufficient to show that

$$
\left\|I-\Pi_{T} R_{\epsilon h}\right\|_{\mathcal{L}\left(\Lambda_{h}^{k}\left(T^{\star}\right), \Lambda_{h}^{k}(T)\right)} \leq c \epsilon,
$$


uniformly over $T \in \mathcal{T}_{h}$ and over $h$. To prove these we shall employ scaling.

Let $\Phi(x)=\left(x-x_{0}\right) / h$ where $x_{0}$ is the first vertex of $T$. Thus $\Phi$ maps $T$ onto a simplex $\hat{T}$ with a vertex at the origin and diameter bounded above and below by positive constants depending only on $C_{\text {mesh }}$. It also maps $T^{\star}$ onto $\hat{T}^{\star}:=\Phi_{T}\left(T^{\star}\right)$. See Figure 5.3. Then $\Phi^{*-1} \Pi_{h} \Phi^{*}: \Lambda^{k}(\hat{T}) \rightarrow \Lambda_{h}^{k}(\hat{T})$

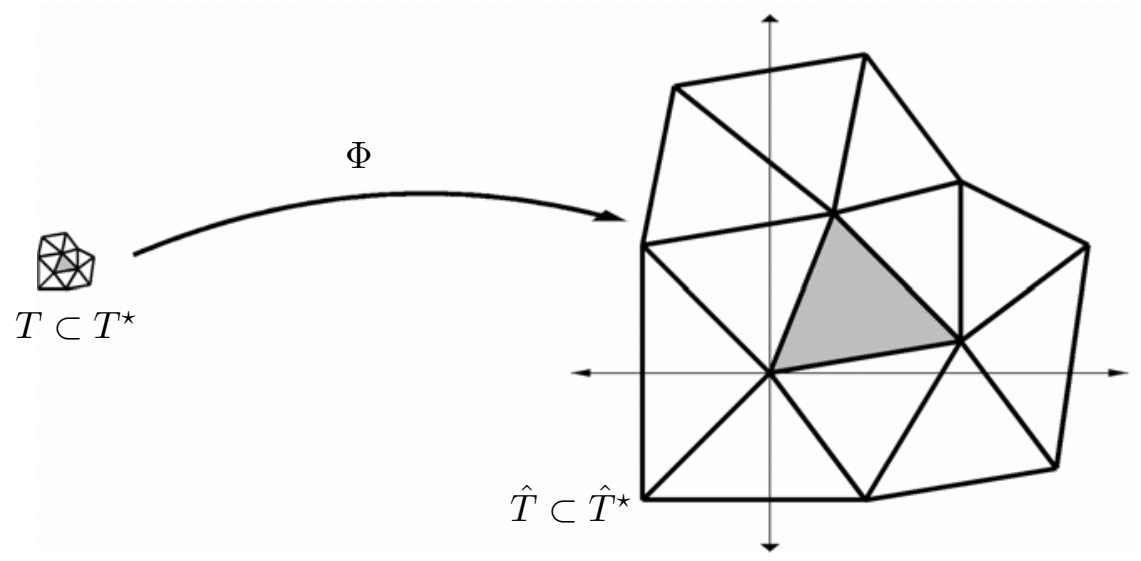

Figure 5.3. Scaling of a simplex and its macroelement.

is just the canonical projection $\Pi_{\hat{T}}$ onto the polynomial space $\Lambda_{h}^{k}(\hat{T})$, and $\Phi^{*-1} R_{\epsilon h} \Phi^{*}: L^{2} \Lambda^{k}\left(\hat{T}^{\star}\right) \rightarrow \Lambda_{h}^{k}(\hat{T})$ is just the smoothing operator $R_{\epsilon}$ (because of our choice of regularization parameter $\epsilon h$, proportional to $h$ ). Thus we find that

$$
\left\|\Pi_{\hat{T}} R_{\epsilon}\right\|_{\mathcal{L}\left(L^{2} \Lambda^{k}\left(\hat{T}^{\star}\right), L^{2} \Lambda^{k}(\hat{T})\right)}=\left\|\Pi_{T} R_{\epsilon h}\right\|_{\mathcal{L}\left(L^{2} \Lambda^{k}\left(T^{\star}\right), L^{2} \Lambda^{k}(T)\right)}
$$

and

$$
\left\|I-\hat{\Pi}_{\hat{T}} R_{\epsilon}\right\|_{\mathcal{L}\left(\Lambda_{h}^{k}\left(\hat{T}^{\star}\right), \Lambda_{h}^{k}(\hat{T})\right)}=\left\|I-\Pi_{T} R_{\epsilon h}\right\|_{\mathcal{L}\left(\Lambda_{h}^{k}\left(T^{\star}\right), \Lambda_{h}^{k}(T)\right)} .
$$

Thus we must show that for fixed $\epsilon$,

$$
\left\|\Pi_{\hat{T}} R_{\epsilon}\right\|_{\mathcal{L}\left(L^{2} \Lambda^{k}\left(\hat{T}^{\star}\right), L^{2} \Lambda^{k}(\hat{T})\right)} \leq c(\epsilon),
$$

uniformly over $T \in \mathcal{T}_{h}$ and over $h$, and that

$$
\left\|I-\Pi_{\hat{T}} R_{\epsilon}\right\|_{\mathcal{L}\left(\Lambda_{h}^{k}\left(\hat{T}^{\star}\right), \Lambda_{h}^{k}(\hat{T})\right)} \leq c \epsilon,
$$

uniformly over $T \in \mathcal{T}_{h}, h$, and $\epsilon$.

For such $T$ and $h$, the configuration of simplices in $\hat{T}^{\star}$ varies over a compact set, and hence it is sufficient to show (5.9) for any single simplex $T$ with a macroelement neighbourhood $T^{\star}$. But this is evident: $R_{\epsilon}$ is bounded $L^{2} \Lambda^{k}\left(\hat{T}^{\star}\right) \rightarrow C \Lambda^{k}(\hat{T})$ (though not uniformly in $\epsilon$ ) and $\Pi_{\hat{T}}$ is bounded $C \Lambda^{k}(\hat{T}) \rightarrow \Lambda_{h}^{k}(\hat{T})$. This proves (5.9) and so Lemma (5.4).

Finally, to prove (5.10) and so Lemma (5.5), We will derive (5.10) from a 
more general bound. We will show that there is a constant $c$, independent of $\epsilon$, such that

$$
\left\|\Pi_{\hat{T}}\left(I-R_{\epsilon}\right) \omega\right\|_{L^{2} \Lambda^{k}(\hat{T})} \leq c \epsilon \sum_{T^{\prime} \in \mathcal{T}(\hat{T})}\|\omega\|_{W^{1, \infty}\left(T^{\prime}\right)}
$$

for all $\omega \in H \Lambda^{k}\left(\hat{T}^{\star}\right)$ such that $\left.\omega\right|_{T^{\prime}} \in H^{1} \Lambda^{k}\left(T^{\prime}\right)$ for $T^{\prime} \in \mathcal{T}(\hat{T})$. Here $\mathcal{T}(\hat{T})$ denotes the set of $n$ simplexes which defines $\hat{T}^{\star}$. In fact, (5.11) will immediately imply (5.10) since $\Pi_{\hat{T}} \omega=\omega$ and

$$
\sum_{T^{\prime} \in \mathcal{T}(\hat{T})}\|\omega\|_{W^{1, \infty}\left(T^{\prime}\right)} \leq c\|\omega\|_{L^{2} \Lambda^{k}\left(\hat{T}^{\star}\right)}
$$

for any $\omega \in \Lambda_{h}^{k}\left(\hat{T}^{\star}\right)$.

In order to establish (5.11) recall that the space $\Lambda_{h}^{k}(\hat{T})$ is either of the form $\mathcal{P}_{r} \Lambda^{k}(\hat{T})$ or $\mathcal{P}_{r}^{-} \Lambda^{k}(\hat{T})$ for a suitable $r \geq 1$. As a consequence of the degrees of freedom for these spaces, given by Theorem 4.10 and Theorem 4.14, to prove (5.11) it is enough to show that for a given $f \in \Delta(\hat{T})$, with $\operatorname{dim} f \geq k$, and $\eta \in \Lambda^{\operatorname{dim} f-k}(f)$ we have

$$
\left|\int_{f}\left(I-R_{\epsilon}\right) \omega \wedge \eta\right| \leq c \epsilon \sum_{T^{\prime} \in \mathcal{T}(\hat{T})}\|\omega\|_{W^{1, \infty}\left(T^{\prime}\right)}
$$

for all $\omega \in H \Lambda^{k}\left(\hat{T}^{\star}\right)$ such that $\left.\omega\right|_{T^{\prime}} \in H^{1} \Lambda^{k}\left(T^{\prime}\right)$ for $T^{\prime} \in \mathcal{T}(\hat{T})$. Here the constant $c$ is independent of $\epsilon$ and $\omega$, but it is allowed to depend on the test function $\eta$.

To show this bound assume that $\omega \in H \Lambda^{k}\left(\hat{T}^{\star}\right) \cap H^{1} \Lambda^{k}\left(T^{\prime}\right), T^{\prime} \in \mathcal{T}(\hat{T})$. We will decompose the $f$ into $f_{\epsilon}$ and $f \backslash f_{\epsilon}$, where

$$
f_{\epsilon}=\{x \in f \mid \operatorname{dist}(x, \partial f) \geq C \epsilon\} .
$$

Here the constant $C>0$ is chosen such that for any point $x \in f_{\epsilon}$, the ball of radius $\epsilon$ with center at $x$ will only intersect the elements of $\mathcal{T}(\hat{T})$ which has $f$ as a subsimplex. A consequence of this construction is that if $x \in f_{\epsilon}$ and $v_{1}, v_{2}, \ldots, v_{k}$ are unit tangent vectors to $f$, then $\omega_{y}\left(v_{1}, \ldots, v_{k}\right)$ is continuous for $|x-y| \leq \epsilon$, and

$$
\left|\omega_{x}\left(v_{1}, \ldots, v_{k}\right)-\omega_{y}\left(v_{1}, \ldots, v_{k}\right)\right| \leq \epsilon \sum_{T^{\prime} \in \mathcal{T}(\hat{T})}\|\omega\|_{W^{1, \infty}\left(T^{\prime}\right)} .
$$

However, this implies that

$$
\left|\int_{f_{\epsilon}}\left(I-R_{\epsilon}\right) \omega \wedge \eta\right| \leq c \epsilon \sum_{T^{\prime} \in \mathcal{T}(\hat{T})}\|\omega\|_{W^{1, \infty}\left(T^{\prime}\right)},
$$

where the constant $c$ is independent of $\epsilon$ and $\omega$. Finally, it is straightforward 
to see that

$$
\left|\int_{f \backslash f_{\epsilon}}\left(I-R_{\epsilon}\right) \omega \wedge \eta\right| \leq\left|\int_{f \backslash f_{\epsilon}} \omega \wedge \eta\right|+\left|\int_{f \backslash f_{\epsilon}} R_{\epsilon} \omega \wedge \eta\right| \leq c \epsilon\|\omega\|_{L^{\infty} \Lambda^{k}\left(\hat{T}^{\star}\right)} .
$$

Hence we have verified the bound (5.12).

\subsection{Discrete de Rham complexes}

As in Section 3.5, the spaces $\mathcal{P}_{r} \Lambda^{k}\left(\mathcal{T}_{h}\right)$ and $\mathcal{P}_{r}^{-} \Lambda^{k}\left(\mathcal{T}_{h}\right)$ lead to a collection of discrete de Rham complexes, essentially $2^{n-1}$ for each polynomial degree. To this end, we observe that

$$
\mathrm{d} \mathcal{P}_{r} \Lambda^{k}\left(\mathcal{T}_{h}\right) \subset \mathcal{P}_{r-1} \Lambda^{k+1}\left(\mathcal{T}_{h}\right) \subset \mathcal{P}_{r}^{-} \Lambda^{k+1}\left(\mathcal{T}_{h}\right),
$$

and

$$
\mathrm{d} \mathcal{P}_{r}^{-} \Lambda^{k}\left(\mathcal{T}_{h}\right) \subset \mathcal{P}_{r-1} \Lambda^{k+1}\left(\mathcal{T}_{h}\right) \subset \mathcal{P}_{r}^{-} \Lambda^{k+1}\left(\mathcal{T}_{h}\right)
$$

This leads to discrete complexes of the form

$$
0 \rightarrow \Lambda_{h}^{0} \stackrel{\mathrm{d}}{\rightarrow} \Lambda_{h}^{1} \stackrel{\mathrm{d}}{\rightarrow} \cdots \stackrel{\mathrm{d}}{\rightarrow} \Lambda_{h}^{n} \rightarrow 0,
$$

where for each map of the form $\Lambda_{h}^{k} \stackrel{\mathrm{d}}{\rightarrow} \Lambda_{h}^{k+1}$, we can substitute one of the four choices

$$
\begin{array}{cc}
\mathcal{P}_{r} \Lambda^{k}\left(\mathcal{T}_{h}\right) \stackrel{\mathrm{d}}{\rightarrow} \mathcal{P}_{r-1} \Lambda^{k+1}\left(\mathcal{T}_{h}\right), & \mathcal{P}_{r} \Lambda^{k}\left(\mathcal{T}_{h}\right) \stackrel{\mathrm{d}}{\rightarrow} \mathcal{P}_{r}^{-} \Lambda^{k+1}\left(\mathcal{T}_{h}\right), \\
\mathcal{P}_{r}^{-} \Lambda^{k}\left(\mathcal{T}_{h}\right) \stackrel{\mathrm{d}}{\rightarrow} \mathcal{P}_{r}^{-} \Lambda^{k+1}\left(\mathcal{T}_{h}\right), & \mathcal{P}_{r}^{-} \Lambda^{k}\left(\mathcal{T}_{h}\right) \stackrel{\mathrm{d}}{\rightarrow} \mathcal{P}_{r-1} \Lambda^{k+1}\left(\mathcal{T}_{h}\right)
\end{array}
$$

Each complex so obtained is a subcomplex of the $L^{2}$ de Rham complex (2.6). Making use of the smoothed projections, we obtain in each case a commuting diagram

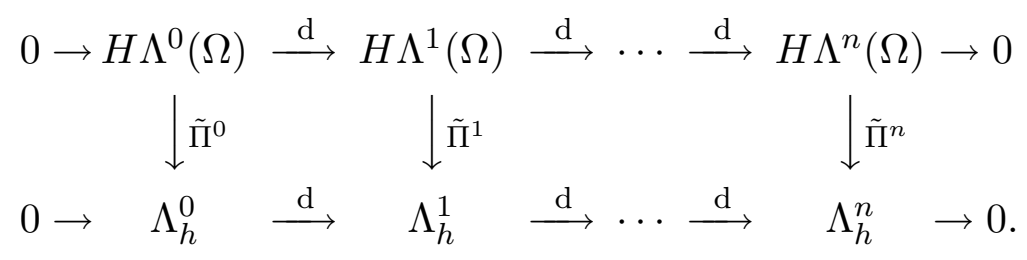

Thus the projections give a cochain projection from the de Rham sequence to the discrete de Rham sequence, and so induce a surjection on cohomology. In fact as we shall now prove, following Christiansen (2005), this is in each case an isomorphism on cohomology.

The simplest finite element de Rham complex is the complex of Whitney forms,

$$
0 \rightarrow \mathcal{P}_{1}^{-} \Lambda^{0}\left(\mathcal{T}_{h}\right) \stackrel{\mathrm{d}}{\rightarrow} \mathcal{P}_{1}^{-} \Lambda^{1}\left(\mathcal{T}_{h}\right) \stackrel{\mathrm{d}}{\rightarrow} \cdots \stackrel{\mathrm{d}}{\rightarrow} \mathcal{P}_{1}^{-} \Lambda^{n}\left(\mathcal{T}_{h}\right) \rightarrow 0 .
$$

That the cohomology of this complex is isomorphic with the de Rham cohomology is a known, but deep result. It follows from de Rham's theorem, 
since the cohomology of the complex of Whitney forms is equal to the simplicial cohomology associated with the triangulation $\mathcal{T}_{h}$, de Rham's theorem states that this simplicial cohomology is isomorphic to the de Rham cohomology.

Considering the Whitney forms complex (5.14) as a subcomplex of (5.13), the canonical projections $\Pi_{h}$ define cochain projections. Note that the $\Pi_{h}$ are defined on the finite element spaces $\Lambda_{h}^{k}$, because all of the trace moments they require are single-valued on $\Lambda_{h}^{k}$. From the commuting diagram

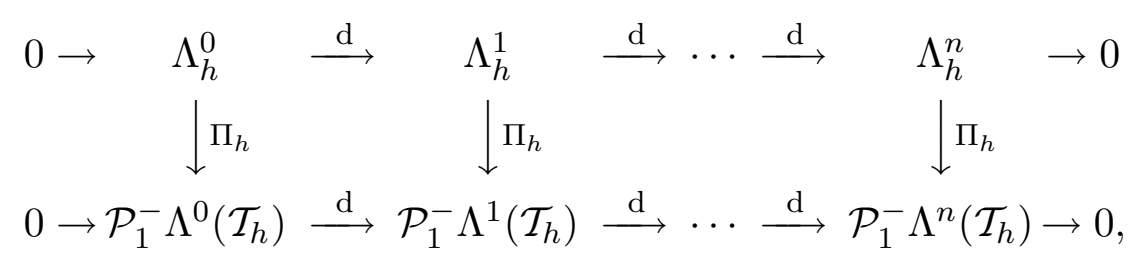

we conclude that the cohomology of the top row, which we have already seen to be an image of the de Rham cohomology, maps onto the cohomology of the bottom row, which is isomorphic to the de Rham cohomology. Hence the dimension of all the corresponding cohomology groups are equal and both cochain projections induce an isomorphism on cohomology.

\subsection{Discrete Hodge decompositions and discrete Poincaré inequality}

We close this section with the discrete analogue of the Hodge decomposition and of Poincaré's inequality.

Discrete Hodge decomposition and discrete harmonic functions

Let

$$
\mathfrak{Z}_{h}^{k}=\left\{\omega \in \Lambda_{h}^{k} \mid \mathrm{d} \omega=0\right\}, \quad \mathfrak{B}_{h}^{k}=\mathrm{d} \Lambda_{h}^{k-1},
$$

denote the spaces of finite element cycles and boundaries. We have $\mathfrak{B}_{h}^{k} \subset \mathfrak{Z}_{h}^{k}$ so $\mathfrak{Z}_{h}^{k \perp} \subset \mathfrak{B}_{h}^{k \perp}$, where the orthogonal complements are taken within the space $\Lambda_{h}^{k}$ with respect to the $L^{2} \Lambda^{k}$-norm (or the $H \Lambda^{k}$-norm, which gives the same result). The orthogonal complement of $\mathfrak{Z}_{h}^{k \perp}$ inside $\mathfrak{B}_{h}^{k \perp}$ is

$$
\mathfrak{H}_{h}^{k}:=\mathfrak{B}_{h}^{k \perp} \cap \mathfrak{Z}_{h}^{k}=\left\{\omega \in \Lambda_{h}^{k} \mid \mathrm{d} \omega=0,\langle\omega, \mathrm{d} \tau\rangle=0 \forall \tau \in \Lambda_{h}^{k-1}\right\},
$$

the space of discrete harmonic forms. We have seen above that this space has the dimension of the $k$ th de Rham cohomology space, which is the $k$ th Betti number of the domain. We note that $\mathfrak{B}_{h}^{k} \subset \mathfrak{B}^{k}$ and $\mathfrak{Z}_{h}^{k} \subset \mathfrak{Z}^{k}$, but $\mathfrak{Z}_{h}^{k \perp}$ is not generally contained in $\mathfrak{Z}^{k \perp}$ and $\mathfrak{H}_{h}^{k}$ is not generally contained in $\mathfrak{H}^{k}$.

The discrete Hodge decomposition is a simple consequence of the definitions:

$$
\Lambda_{h}^{k}=\mathfrak{B}_{h}^{k} \oplus \mathfrak{B}_{h}^{k \perp}=\mathfrak{B}_{h}^{k} \oplus \mathfrak{H}_{h}^{k} \oplus \mathfrak{Z}_{h}^{k \perp} .
$$

We now make some important observations about discrete harmonic forms. 
The following theorem shows that they can be computed as the elements of the null space of a finite element matrix.

Theorem 5.7. Consider the homogeneous linear system: find $\left(\sigma_{h}, u_{h}\right) \in$ $\Lambda_{h}^{k-1} \times \Lambda_{h}^{k}$ such that

$$
\begin{gathered}
\left\langle\sigma_{h}, \tau\right\rangle=\left\langle\mathrm{d} \tau, u_{h}\right\rangle, \quad \tau \in \Lambda_{h}^{k-1}, \\
\left\langle\mathrm{~d} \sigma_{h}, v\right\rangle+\left\langle\mathrm{d} u_{h}, \mathrm{~d} v\right\rangle=0, \quad v \in \Lambda_{h}^{k} .
\end{gathered}
$$

Then $\left(\sigma_{h}, u_{h}\right)$ is a solution if and only if $\sigma_{h}=0$ and $u_{h} \in \mathfrak{H}_{h}^{k}$.

Proof. Clearly $\left(0, u_{h}\right)$ is a solution if $u_{h} \in \mathfrak{H}_{h}^{k}$. On the other hand, if $\left(\sigma_{h}, u_{h}\right)$ is a solution, by taking $\tau=\sigma_{h}, v=u_{h}$, and combining the two equations, we find that $\left\|\sigma_{h}\right\|^{2}+\left\|\mathrm{d} u_{h}\right\|^{2}=0$, so that $\sigma_{h}=0$ and $\mathrm{d} u_{h}=0$. Then the first equation implies that $\left\langle\mathrm{d} \tau, u_{h}\right\rangle=0$ for all $\tau \in \Lambda_{h}^{k-1}$, so indeed $u_{h} \in \mathfrak{H}_{h}^{k}$.

We know that the space of discrete harmonic $k$-forms has the same dimension as the space of harmonic $k$-forms. In the next theorem we show that as well the discrete harmonic forms provide good approximation of the harmonic forms. In it we use the smoothed projection operator $\tilde{\Pi}_{h}$, but, as can be seen from the proof, any projection operator which commutes with d could be used just as well.

Theorem 5.8. Let $\sigma \in \mathfrak{H}^{k}$. Then there exists $\sigma_{h} \in \mathfrak{H}_{h}^{k}$ such that

$$
\left\|\sigma-\sigma_{h}\right\| \leq\left\|\sigma-\tilde{\Pi}_{h} \sigma\right\|
$$

Proof. First we show that there exists a unique $\left(\sigma_{h}, u_{h}\right) \in \Lambda_{h}^{k} \times \mathfrak{B}_{h}^{k+1}$ such that

$$
\begin{gathered}
\left\langle\sigma_{h}, \tau\right\rangle-\left\langle\mathrm{d} \tau, u_{h}\right\rangle=\langle\sigma, \tau\rangle, \quad \tau \in \Lambda_{h}^{k}, \\
\left\langle\mathrm{~d} \sigma_{h}, v\right\rangle=0, \quad v \in \mathfrak{B}_{h}^{k+1} .
\end{gathered}
$$

This is a finite-dimensional linear system, so we just need to show that if $\left(\sigma_{h}, u_{h}\right)$ is a solution when $\sigma=0$, then $\sigma_{h}=0$ and $u_{h}=0$. Choosing $\tau=\sigma_{h}$ and $v=u_{h}$, we get that $\sigma_{h}=0$, and then that $\left\langle\mathrm{d} \tau, u_{h}\right\rangle=0$ for all $\tau \in \Lambda_{h}^{k}$, i.e., $u_{h} \in \mathfrak{B}_{h}^{k+1 \perp}$. Thus $u_{h}=0$.

Next we show that for the solution of (5.16), $\sigma_{h} \in \mathfrak{H}_{h}^{k}$. The second equation immediately gives $\mathrm{d} \sigma_{h}=0$, i.e., $\sigma_{h} \in \mathfrak{Z}_{h}^{k}$. Taking $\tau=\mathrm{d} \rho, \rho \in$ $\Lambda_{h}^{k-1}$ we get

$$
\left\langle\sigma_{h}, \mathrm{~d} \rho\right\rangle=\langle\sigma, \mathrm{d} \rho\rangle=0, \quad \rho \in \Lambda_{h}^{k-1},
$$

where the last equation holds because $\sigma$ is harmonic. Thus $\sigma_{h} \in \mathfrak{B}_{h}^{k \perp}$, i.e., $\sigma_{h} \in \mathfrak{H}_{h}^{k}$.

Finally, we have from the first equation in (5.16) that

$$
\left\langle\sigma-\sigma_{h}, \tilde{\Pi}_{h} \sigma-\sigma_{h}\right\rangle=\left\langle\mathrm{d}\left(\sigma_{h}-\tilde{\Pi}_{h} \sigma\right), u_{h}\right\rangle=0
$$


since $\mathrm{d} \sigma_{h}=0$ and $\mathrm{d} \tilde{\Pi}_{h} \sigma=\tilde{\Pi}_{h} \mathrm{~d} \sigma=0$. The inequality (5.15) follows immediately.

It is also the case, as we show below, that a discrete harmonic $k$-form can be approximated well by a harmonic $k$-form.

Lemma 5.9. Let $p \in \mathfrak{H}_{h}^{k}$ be a discrete harmonic $k$-form. There exists a $r \in \mathfrak{H}^{k}$ such that $\|r\| \leq\|p\|$ and

$$
\|p-r\| \leq\left\|\left(I-\tilde{\Pi}_{h}\right) r\right\|
$$

Proof. We define $r=p-\mathrm{d} \sigma$, where $\mathrm{d} \sigma \in \mathfrak{B}^{k}$ is the $L^{2}$-projection of $p$ onto $\mathfrak{B}^{k}$, i.e.

$$
\langle\mathrm{d} \sigma, \mathrm{d} \tau\rangle=\langle p, \mathrm{~d} \tau\rangle, \quad \tau \in H \Lambda^{k-1}(\Omega) .
$$

Note that $r \in \mathfrak{Z}^{k}$ since both $p$ and $\mathrm{d} \sigma$ belongs to this space. On the other hand, the definition of $\sigma$ implies that

$$
\langle r, \mathrm{~d} \tau\rangle=0, \quad \tau \in H \Lambda^{k-1}(\Omega) .
$$

Hence, $r \in \mathfrak{Z}^{k} \cap \mathfrak{B}^{k \perp}=\mathfrak{H}^{k}$. Furthermore, the bound $\|r\|=\|p-\mathrm{d} \sigma\| \leq\|p\|$ is a consequence of the fact that $\mathrm{d} \sigma$ is the $L^{2}$-projection of $p$. Finally, to derive the error bound, observe that since $p \in \mathfrak{H}_{h}^{k}$ we have

$$
\langle r-p, \mathrm{~d} \tau\rangle=-\langle p, \mathrm{~d} \tau\rangle=0, \quad \tau \in \Lambda_{h}^{k-1} .
$$

Therefore,

$$
\begin{aligned}
\|r-p\|^{2} & =\langle r-p, \mathrm{~d} \sigma\rangle=\left\langle r-p, \mathrm{~d}\left(I-\tilde{\Pi}_{h}\right) \sigma\right\rangle \\
& \leq\|r-p\|\left\|\left(I-\tilde{\Pi}_{h}\right) \mathrm{d} \sigma\right\|=\|r-p\|\left\|\left(I-\tilde{\Pi}_{h}\right) r\right\|,
\end{aligned}
$$

which gives the desired bound for $\|r-p\|$.

The following approximation result, relating forms in $\mathfrak{Z}_{h}^{k \perp}$ to forms in $\mathfrak{Z}^{k \perp}$ will also prove useful.

Lemma 5.10. If $u \in \mathfrak{Z}_{h}^{k \perp}$ and $w \in \mathfrak{Z}^{k \perp}$ satisfies $\mathrm{d} w=\mathrm{d} u$, then

$$
\|u-w\| \leq\left\|w-\tilde{\Pi}_{h} w\right\| .
$$

Proof. $\quad$ Since $w \in \mathfrak{Z}^{k \perp}$, there exists $z \in \stackrel{\circ}{\mathfrak{B}}^{k+1}$ such that $w=\delta z$, i.e.,

$$
\langle w, v\rangle=\langle\mathrm{d} v, z\rangle, \quad v \in H \Lambda^{k}(\Omega) .
$$

Similarly, since $u \in \mathfrak{Z}_{h}^{k \perp}$, there exists $z_{h} \in \mathfrak{B}_{h}^{k+1}$ such that

$$
\langle u, v\rangle=\left\langle\mathrm{d} v, z_{h}\right\rangle, \quad v \in \Lambda_{h}^{k}
$$

Hence,

$$
\langle w-u, v\rangle=\left\langle\mathrm{d} v, z-z_{h}\right\rangle, \quad v \in \Lambda_{h}^{k}
$$


Choosing $v=\tilde{\Pi}_{h} w-u$ and noting that $\mathrm{d} v=0$, we get

$$
\|w-u\|^{2}=\left\langle w-u, w-\tilde{\Pi}_{h} w\right\rangle \leq\|w-u\|\left\|w-\tilde{\Pi}_{h} w\right\| .
$$

The result follows immediately.

A discrete Poincaré inequality

Using Lemma 5.10, we prove the analogue of (2.17).

Theorem 5.11. There is a positive constant $c$, independent of $h$, such that

$$
\|\omega\| \leq c\|\mathrm{~d} \omega\|, \quad \omega \in \mathfrak{Z}_{h}^{k \perp} .
$$

Proof. Define $\eta \in \mathfrak{Z}^{k \perp} \subset H \Lambda^{k}(\Omega)$ by $\mathrm{d} \eta=\mathrm{d} \omega$ (so $\eta$ is the $L^{2}$-projection of $\omega$ into $\mathfrak{Z}^{k \perp}$.) By (2.17),

$$
\|\eta\| \leq c\|\mathrm{~d} \omega\| .
$$

Hence, it is enough to show that $\|\omega\| \leq c\|\eta\|$. But this follows immediately from Lemma 5.10 and the boundedness of $\tilde{\Pi}_{h}$.

\section{Differential forms with values in a vector space}

In Section 11 at the end of this paper, in which we study discretizations of the equations of elasticity, we will need to use differential forms with values in a vector space. We introduce the necessary ideas here, which are straightforward extensions of the material in Section 2. Federer (1969) is one reference for this material. At the end of this section we consider a particular operator acting on vector- and bivector-valued algebraic forms, and establish some properties which will be needed later.

Let $V$ and $W$ be finite-dimensional vector spaces. We then define the space $\operatorname{Alt}^{k}(V ; W)$ of alternating $k$-linear forms on $V$ with values in $W$. This is a vector space of dimension $(\underset{k}{\operatorname{dim} V}) \operatorname{dim} W$. There is natural identification of $\left(\operatorname{Alt}^{k} V\right) \otimes W$ with $\operatorname{Alt}^{k}(V ; W)$ given by $(\omega \otimes w)\left(v_{1}, \ldots, v_{k}\right)=\omega\left(v_{1}, \ldots, v_{k}\right) w, \quad \omega \in \operatorname{Alt}^{k} V, w \in W, v_{1}, \ldots, v_{k} \in V$.

For $k=0$ and 1 , we have $\operatorname{Alt}^{0}(V ; W)=W$ and $\operatorname{Alt}^{1}(V ; W)=\mathcal{L}(V, W)$, the space of linear operators from $V \rightarrow W$. Most of the definitions of Section 2.1 carry over without difficulty. For some we require an inner product on $W$, which we shall assume is given (and denoted with a dot). For example, the exterior product maps $\operatorname{Alt}^{j}(V ; W) \times \operatorname{Alt}^{k}(V ; W) \rightarrow \operatorname{Alt}^{j+k} V$ (the range space is scalar-valued). It is defined by the analogue of (2.1)

$$
\begin{aligned}
& (\omega \wedge \eta)\left(v_{1}, \ldots, v_{j+k}\right) \\
& \quad=\sum_{\sigma}(\operatorname{sign} \sigma) \omega\left(v_{\sigma(1)}, \ldots, v_{\sigma(j)}\right) \cdot \eta\left(v_{\sigma(j+1)}, \ldots, v_{\sigma(j+k)}\right), \quad v_{i} \in V
\end{aligned}
$$


where the sum is again over all permutations $\sigma$ of $\{1, \ldots, j+k\}$, for which $\sigma(1)<\sigma(2)<\cdots \sigma(j)$ and $\sigma(j+1)<\sigma(j+2)<\cdots \sigma(j+k)$. Assuming that $V$ also has an inner product, we get an inner product on $\operatorname{Alt}^{k}(V ; W)$ in analogy with (2.2):

$$
\langle\omega, \eta\rangle=\sum_{\sigma} \omega\left(v_{\sigma(1)}, \ldots, v_{\sigma(k)}\right) \cdot \eta\left(v_{\sigma(1)}, \ldots, v_{\sigma(k)}\right), \quad \omega, \eta \in \operatorname{Alt}^{k}(V ; W),
$$

where the sum is over increasing sequences $\sigma:\{1, \ldots, k\} \rightarrow\{1, \ldots, n\}$ and $v_{1}, \ldots, v_{n}$ is any orthonormal basis. Assuming also an orientation on $V$, the Hodge star operation is again defined by

$$
\omega \wedge \mu=\langle\star \omega, \mu\rangle \text { vol, } \quad \omega \in \operatorname{Alt}^{k}(V ; W), \mu \in \operatorname{Alt}^{n-k}(V ; W) .
$$

Both sides of this equation are elements of the 1-dimensional space $\operatorname{Alt}^{n} V$ of real-valued $n$-forms on $V$.

For a manifold $\Omega$, we define the space $\Lambda^{k}(\Omega ; W)$ of differential $k$-forms with values in $W$ in the obvious way, i.e., as forms $\omega$ on $\Omega$, such that at each point $x \in \Omega, \omega_{x} \in \operatorname{Alt}^{k}\left(T_{x} \Omega ; W\right)$. By taking the tensor product of the de Rham complex with $W$ we get the vector-valued de Rham complex

$$
0 \rightarrow \Lambda^{0}(\Omega ; W) \stackrel{\mathrm{d}}{\rightarrow} \Lambda^{1}(\Omega ; W) \stackrel{\mathrm{d}}{\rightarrow} \cdots \stackrel{\mathrm{d}}{\rightarrow} \Lambda^{n}(\Omega ; W) \rightarrow 0 .
$$

Here d represents the $W$-valued exterior derivative $\mathrm{d} \otimes \mathrm{id}_{W}$ where $\mathrm{d}$ is the ordinary exterior derivative. The cohomology is just the tensor product of the ordinary de Rham cohomology with $W$.

When $\Omega$ is an open subset of $\mathbb{R}^{n}$ (the only case we require), we can write an arbitrary element of $\Lambda^{k}(\Omega ; W)$ as $\sum a_{\sigma} \mathrm{d} x_{\sigma(1)} \wedge \cdots \wedge \mathrm{d} x_{\sigma(k)}$ with the $a_{\sigma}$ functions from $\Omega \rightarrow W$. In Section 11 we will use two different vector spaces $W$, namely $V=\mathbb{R}^{n}$ (to be thought of as the tangent space to $\Omega$ at any point; we use the linear structure and the Euclidean inner product on $V$ but our approach is basis independent) and $V \wedge V$, the space of bivectors, defined in Section 2.1 and identifiable with the space of skew-symmetric linear operators on $V$.

In treating the equations of elasticity on a domain $\Omega \subset \mathbb{R}^{n}$, we shall represent the stress as an element $\sigma \in \operatorname{Alt}^{n-1}(\Omega ; V)$. This is natural, because the stress is a quantity that, when integrated over surfaces (submanifolds of dimension $n-1$ ), gives the force vector (or covector - in view of the inner product, we will not draw this distinction). Let us relate this to the usual definition of the stress, a second-order tensor (or matrix) defined at each point $x \in \Omega$, which, when multiplied by the normal vector to a surface passing through the point, yields the surface force density acting on the surface. The tensor of course represents an element of $\mathcal{L}(V, V)=\operatorname{Alt}^{1}(V ; V)$. That operator is simply $\star \sigma_{x}$.

To close this section, we consider the operator $S=S_{k}: \operatorname{Alt}^{k}(V ; V) \rightarrow$ 
$\operatorname{Alt}^{k+1}(V ; V \wedge V)$, defined by

$$
\begin{array}{r}
(S \omega)\left(v_{1}, \ldots, v_{k+1}\right)=\sum_{j=1}^{k+1}(-1)^{j+1} v_{j} \wedge \omega\left(v_{1}, \ldots, \hat{v}_{j}, \ldots, v_{k+1}\right) \\
v_{1}, \ldots, v_{k+1} \in V
\end{array}
$$

for $V$ an inner product space of dimension $n$. Of particular importance for our work in elasticity are the cases $k=n-1$ and $k=n-2$. The operator $S_{n-1}: \Lambda^{n-1}(V ; V) \rightarrow \Lambda^{n}(V ; V \wedge V)$, in particular, is a familiar operator in disguise. This is revealed by composing with the Hodge star isomorphism on both sides.

Proposition 6.1. The composition

$$
\operatorname{Alt}^{1}(V ; V) \stackrel{\star}{\rightarrow} \operatorname{Alt}^{n-1}(V ; V) \stackrel{S}{\rightarrow} \operatorname{Alt}^{n}(V ; V \wedge V) \stackrel{\star}{\rightarrow} \operatorname{Alt}^{0}(V ; V \wedge V)
$$

is equal to $(-1)^{n} 2 \mathrm{skw}$.

Proof. Let $v, w \in V$, and view $v \otimes w$ as an element of $\operatorname{Alt}^{1}(V ; V)$. We shall show that $\star S(\star(v \otimes w))=(-1)^{n} v \wedge w$. Since such elements span $\operatorname{Alt}^{1}(V ; V)$, this gives the result. The calculation is straightforward. Let $e_{1}, \ldots, e_{n}$ be a positively oriented orthonormal basis for $V$. Then

$$
\begin{aligned}
\star S(\star(v \otimes w)) & =S(\star(v \otimes w))\left(e_{1}, \ldots, e_{n}\right) \\
& =\sum_{j=1}^{n}(-1)^{j+1} e_{j} \wedge(\star(v \otimes w))\left(e_{1}, \ldots, \hat{e}_{j}, \ldots, e_{n}\right) \\
& =\sum_{j=1}^{n}(-1)^{j+1} e_{j} \wedge(-1)^{n-j}(v \otimes w)\left(e_{j}\right) \\
& =(-1)^{n+1} \sum_{j=0}^{n} e_{j} \wedge v\left(w \cdot e_{j}\right)=(-1)^{n} \sum_{j=1}^{n} v \wedge w,
\end{aligned}
$$

where we have substituted $w$ for $\sum\left(w_{j} \cdot e_{j}\right) e_{j}$ in the last step.

Finally we consider the operator $S_{k}$ for $k=n-2$. In this case the dimensions of the domain and range coincide:

$$
\operatorname{dim} \operatorname{Alt}^{n-2}(V ; V)=\left(\begin{array}{l}
n \\
2
\end{array}\right) n=\operatorname{dim} \operatorname{Alt}^{n-1}(V ; V \wedge V) .
$$

In fact, the operator is an isomorphism. To prove this, we first establish two lemmas.

Lemma 6.2. Let $e_{1}, \ldots, e_{n}$ be a positively oriented orthonormal basis of 
$V$, and let $\omega \in \operatorname{Alt}^{n-2}(V ; V)$. Then

$$
\left(S_{n-2} \omega\right)\left(e_{1}, \ldots, \hat{e}_{i}, \ldots, e_{n}\right)=(-1)^{i+1} \sum_{j=1}^{n} e_{j} \wedge(\star \omega)\left(e_{i}, e_{j}\right), \quad i=1, \ldots, n .
$$

Proof. It suffices to prove the case $i=n$, since we may always reorder the basis elements (possibly changing orientation). Then, from the definition of $S_{n-2}$

$$
\begin{aligned}
\left(S_{n-2} \omega\right)\left(e_{1}, \ldots, e_{n-1}\right) & =\sum_{j=1}^{n-1}(-1)^{j+1} e_{j} \wedge \omega\left(e_{1}, \ldots, \hat{e}_{j}, \ldots, e_{n-1}\right) \\
& =\sum_{j=1}^{n-1}(-1)^{j+1}(-1)^{n-j-1} e_{j} \wedge \star \omega\left(e_{j}, e_{n}\right) \\
& =(-1)^{n+1} \sum_{j=1}^{n} e_{j} \wedge \star \omega\left(e_{n}, e_{j}\right)
\end{aligned}
$$

where we have used the fact that $\star \omega\left(e_{n}, e_{n}\right)=0$.

Lemma 6.3. Suppose $\mu \in \operatorname{Alt}^{2}(V ; V)$ satisfies

$$
\sum_{j=1}^{n} e_{j} \wedge \mu\left(e_{i}, e_{j}\right)=0, \quad i=1, \ldots, n,
$$

for some orthonormal basis $e_{1}, \ldots, e_{n}$ of $V$. Then $\mu=0$.

Proof. We may expand $\mu\left(e_{i}, e_{j}\right)=\sum_{k=1}^{n} \mu_{i j k} e_{k}$, for some coefficients $\mu_{i j k} \in$ $\mathbb{R}$ satisfying

$$
\mu_{i j k}=-\mu_{j i k}
$$

Now

$$
0=\sum_{j, k=1}^{n} \mu_{i j k} e_{j} \wedge e_{k}=\sum_{j<k}\left(\mu_{i j k}-\mu_{i k j}\right) e_{j} \wedge e_{k}
$$

whence we conclude

$$
\mu_{i j k}=\mu_{i k j} .
$$

But (6.3) and (6.4) imply that $\mu$ vanishes:

$$
\mu_{i j k}=-\mu_{j i k}=-\mu_{j k i}=\mu_{k j i}=\mu_{k i j}=-\mu_{i k j}=-\mu_{i j k} .
$$

Theorem 6.4. The operator $S_{n-2}: \operatorname{Alt}^{n-2}(V, V) \rightarrow \operatorname{Alt}^{n-1}(V, V \wedge V)$ is an isomorphism.

Proof. If $S_{n-2} \omega=0$ then Lemmas 6.2 and 6.3 imply that $\star \omega$ vanishes, so $\omega$ vanishes. Then $S_{n-2}$ is injective, and since its domain and range have equal dimension, it is an isomorphism. 


\section{PART TWO}

\section{Applications to discretization of differential equations}

\section{The Hodge Laplacian}

In this section, we consider the discretization of boundary value problems associated to the Hodge Laplacian, $\mathrm{d} \delta+\delta \mathrm{d}$, by mixed finite element methods. After first obtaining a mixed variational formulation of these boundary value problems, we then translate to the language of partial differential equations in the case when $n=3$. The aim here is to show that these formulations in this general setting include many of the problems important in applications. The first main result of the section is to establish the well-posedness of the mixed formulation. We then turn to finite element discretization using the finite element spaces developed in the Part 1 of the paper. Using the tools developed for these spaces, we are easily able to establish stability of the mixed finite element approximation. By standard finite element theory, this gives a quasioptimal error estimate for the variables being approximated. It is well known, however, that since this estimate couples together all the variables being approximated, it does not always give the best result for the approximation of each variable separately, and these more refined results are needed in some applications, and in particular for the approximation of the eigenvalue problem associated to the Hodge Laplacian. Hence, we end the section with a detailed error analysis of these mixed finite element methods.

\subsection{Mixed formulation of the Hodge Laplacian}

Let $\Omega$ be a domain in $\mathbb{R}^{n}$ and $0 \leq k \leq n$ an integer. Given $f \in L^{2} \Lambda^{k}(\Omega)$, define $\mathcal{J}: H \Lambda^{k-1}(\Omega) \times H \Lambda^{k}(\Omega) \times \mathfrak{H}^{k} \rightarrow \mathbb{R}$ by

$$
\mathcal{J}(\tau, v, q)=\frac{1}{2}\langle\tau, \tau\rangle-\langle\mathrm{d} \tau, v\rangle-\frac{1}{2}\langle\mathrm{~d} v, \mathrm{~d} v\rangle-\langle v, q\rangle+\langle f, v\rangle .
$$

Then a critical point, $(\sigma, u, p) \in H \Lambda^{k-1}(\Omega) \times H \Lambda^{k}(\Omega) \times \mathfrak{H}^{k}$ of $\mathcal{J}$ is determined by the equations

$$
\begin{aligned}
\langle\sigma, \tau\rangle & =\langle\mathrm{d} \tau, u\rangle, & & \tau \in H \Lambda^{k-1}(\Omega), \\
\langle\mathrm{d} \sigma, v\rangle+\langle\mathrm{d} u, \mathrm{~d} v\rangle+\langle v, p\rangle & =\langle f, v\rangle, & & v \in H \Lambda^{k}(\Omega), \\
\langle u, q\rangle & =0, & & q \in \mathfrak{H}^{k} .
\end{aligned}
$$

In this formulation, $p$ is a Lagrange multiplier corresponding to the constraint given by the third equation of (7.1). However, even if $p$ is eliminated by incorporating this constraint into the space $H \Lambda^{k}(\Omega)$, the critical point 
would still be a saddle point - a minimizer with respect to $\sigma$ and a maximizer with respect to $u$ - and could not generally be obtained from a constrained minimization problem for $\sigma$ via introduction of an additional Lagrange multiplier.

Letting $P_{\mathfrak{H}^{k}}$ denote the $L^{2}$-projection into $\mathfrak{H}^{k}$, equations $(7.1)$ are weak formulations of the equations

$$
\sigma=\delta u, \quad \mathrm{~d} \sigma+\delta \mathrm{d} u+p=f, \quad P_{\mathfrak{H}^{k}} u=0,
$$

respectively, and, since $p=P_{\mathfrak{H}^{k}} f$, together give the Hodge-Laplace problem $(\mathrm{d} \delta+\delta \mathrm{d}) u=f-P_{\mathfrak{H}^{k}} f$, where $\delta$ is the Hodge star operator defined previously. Also implied are the natural boundary conditions that the trace of $\star u$ and the trace of $\star \mathrm{d} u$ on $\partial \Omega$ both must vanish.

If, instead, we seek a critical point $(\sigma, u, p) \in \stackrel{\circ}{H} \Lambda^{k-1}(\Omega) \times \stackrel{\circ}{H} \Lambda^{k}(\Omega) \times \stackrel{\circ}{\mathfrak{H}}^{k}$, then we obtain the essential boundary conditions that the trace of $\sigma$ as a $(k-1)$-form on $\partial \Omega$ and the trace of $u$ as a $k$-form on $\partial \Omega$ both must vanish.

\subsection{Splitting of the mixed formulation}

By using the Hodge decomposition (2.18), we can split the problem (7.1) into three simpler problems. First, we write $f=f_{\mathrm{d}}+f_{\mathfrak{H}}+f_{\delta}$ where $f_{\mathrm{d}} \in$ $\mathfrak{B}^{k}=\mathrm{d}\left(H \Lambda^{k-1}(\Omega)\right), f_{\mathfrak{H}} \in \mathfrak{H}^{k}$, and $f_{\delta} \in \stackrel{\circ}{\mathfrak{B}}^{* k}=\delta \stackrel{\circ}{H}^{*} \Lambda^{k+1}(\Omega)$.

Now let $(\sigma, u, p)$ be a solution of (7.1). From the second equation in (7.1), it follows immediately that $p_{\mathfrak{H}}=f_{\mathfrak{H}}$, and from the third equation it follows that $u \in \mathfrak{H}^{k \perp}$, so $u=u_{\mathrm{d}}+u_{\delta}$ with $u_{\mathrm{d}} \in \mathfrak{B}^{k}$ and $u_{\delta} \in \stackrel{\circ}{\mathfrak{B}}^{* k}$.

Taking $v \in \mathfrak{B}^{* k}$ we find that $u_{\delta} \in \mathfrak{B}^{* k}$ satisfies

$$
\left\langle\mathrm{d} u_{\delta}, \mathrm{d} v\right\rangle=\left\langle f_{\delta}, v\right\rangle, \quad v \in \stackrel{\circ}{\mathfrak{B}}^{* k} .
$$

Taking $v \in \mathfrak{B}^{k}$ we find that $\left(\sigma, u_{\mathrm{d}}\right) \in H \Lambda^{k-1}(\Omega) \times \mathfrak{B}^{k}$ satisfies

$$
\langle\sigma, \tau\rangle=\left\langle\mathrm{d} \tau, u_{\mathrm{d}}\right\rangle, \tau \in H \Lambda^{k-1}(\Omega), \quad\langle\mathrm{d} \sigma, v\rangle=\left\langle f_{\mathrm{d}}, v\right\rangle, v \in \mathfrak{B}^{k} .
$$

The converse reasoning is also straightforward, and so we have the following theorem.

Theorem 7.1. Suppose that $(\sigma, u, p) \in H \Lambda^{k-1}(\Omega) \times H \Lambda^{k}(\Omega) \times \mathfrak{H}^{k}$ solves (7.1) and that $f$ has the Hodge decomposition $f_{\mathrm{d}}+f_{\mathfrak{H}}+f_{\delta}$, with $f_{\mathrm{d}} \in \mathfrak{B}^{k}$, $f_{\mathfrak{H}} \in \mathfrak{H}^{k}, f_{\delta} \in \mathfrak{\circ}^{* k}$. Then $p=f_{\mathfrak{H}}$ and $u$ has the Hodge decomposition $u_{\mathrm{d}}+u_{\delta}$ with $u_{\mathrm{d}} \in \mathfrak{B}^{k}$ and $u_{\delta} \in \mathfrak{B}^{* k}$ where $u_{\delta}$ solves (7.3) and $\left(\sigma, u_{\mathrm{d}}\right)$ solves (7.4). Conversely, if $p=f_{\mathfrak{H}}, u_{\delta} \in \mathfrak{\mathfrak { B }}^{* k}$ solves $(7.3)$, and $\left(\sigma, u_{\mathrm{d}}\right) \in H \Lambda^{k-1}(\Omega) \times \mathfrak{B}^{k}$ solves (7.4), then, setting $u=u_{\mathrm{d}}+u_{\delta},(\sigma, u, p) \in H \Lambda^{k-1}(\Omega) \times H \Lambda^{k}(\Omega) \times \mathfrak{H}^{k}$ solves (7.1).

In this section we consider the solution to the Hodge Laplacian problem (7.1), but our results apply as well to the solution (7.4) and (7.3) since these are just the special cases when $f=f_{\mathrm{d}} \in \mathfrak{B}^{k}$ or $f=f_{\delta} \in \stackrel{\circ}{\mathfrak{B}^{* k}}$. 
Note that (7.4) is a weak formulation of the equations

$$
\sigma_{\mathrm{d}}=\delta u_{\mathrm{d}}, \quad \mathrm{d} \sigma_{\mathrm{d}}=f_{\mathrm{d}}, \quad \mathrm{d} u_{\mathrm{d}}=0,
$$

together with the natural boundary condition that the trace of $\star u_{\mathrm{d}}$ on $\partial \Omega$ vanishes and the side condition that $u_{\mathrm{d}} \perp \mathfrak{H}^{k}$. Eliminating $\sigma_{\mathrm{d}}$ from the system, it becomes

$$
\mathrm{d} \delta u_{\mathrm{d}}=f_{\mathrm{d}}, \quad \mathrm{d} u_{\mathrm{d}}=0,
$$

with the indicated boundary condition and side condition. Analogously, (7.3) is a weak formulation of the equations

$$
\delta \mathrm{d} u_{\delta}=f_{\delta}, \quad \delta u_{\delta}=0,
$$

together with the essential boundary condition that the trace of $\star u_{\delta}$ on $\partial \Omega$ vanishes and the same side condition.

\subsection{Variable coefficients}

We have considered the mixed formulation (7.2) without introducing coefficients. But we may easily generalize to allow coefficents. Let $A: L^{2} \Lambda^{k-1}(\Omega)$ $\rightarrow L^{2} \Lambda^{k-1}(\Omega)$ and $B: L^{2} \Lambda^{k+1}(\Omega) \rightarrow L^{2} \Lambda^{k+1}(\Omega)$ be bounded, symmetric, positive-definite operators with respect to the standard inner products in $L^{2} \Lambda^{k-1}(\Omega)$ and $L^{2} \Lambda^{k+1}(\Omega)$. Then we may define equivalent inner products:

$$
\langle\sigma, \tau\rangle_{A}:=\langle A \sigma, \tau\rangle, \quad\langle\omega, \mu\rangle_{B}:=\langle B \omega, \mu\rangle,
$$

for $\sigma, \tau \in L^{2} \Lambda^{k-1}(\Omega), \omega, \mu \in L^{2} \Lambda^{k+1}(\Omega)$. We may then consider, as a generalization of (7.1), the problem of finding $(\sigma, u, p) \in H \Lambda^{k-1}(\Omega) \times H \Lambda^{k}(\Omega) \times \mathfrak{H}^{k}$ determined by the equations

$$
\begin{gathered}
\langle\sigma, \tau\rangle_{A}=\langle\mathrm{d} \tau, u\rangle, \tau \in H \Lambda^{k-1}(\Omega), \\
\langle\mathrm{d} \sigma, v\rangle+\langle\mathrm{d} u, \mathrm{~d} v\rangle_{B}+\langle v, p\rangle=\langle f, v\rangle, v \in H \Lambda^{k}(\Omega), \\
\langle u, q\rangle=0, q \in \mathfrak{H}^{k} .
\end{gathered}
$$

This is a weak formulation of the differential equations and side condition

$$
A \sigma=\delta u, \quad \mathrm{~d} \sigma+\delta(B \mathrm{~d} u)+p=f, \quad P_{\mathfrak{H}^{k}} u=0,
$$

and the boundary conditions $\operatorname{Tr}(\star u)=0, \operatorname{Tr}[\star(B \mathrm{~d} u)]=0$ on $\partial \Omega$.

We may split the problem as in the previous subsection, and obtain the two reduced problems, namely

$$
A \sigma_{\mathrm{d}}=\delta u_{\mathrm{d}}, \quad \mathrm{d} \sigma_{\mathrm{d}}=f_{\mathrm{d}}, \quad \mathrm{d} u_{\mathrm{d}}=0
$$

and

$$
\delta\left(B \mathrm{~d} u_{\delta}\right)=f_{\delta}, \quad \delta u_{\delta}=0 .
$$

Although these more general problems are important for applications, 
their treatment is no more complicated, except notationally, than the simple case where $A$ and $B$ are the identity, and so we shall continue to consider only that case.

\subsection{Translation to the language of partial differential equations}

Let us consider more concretely the situation in $n=3$ dimensions, identifying the spaces $H \Lambda^{k}(\Omega)$ with function spaces as described in Section 2.3. For $k=3,(7.1)$ becomes: find $\sigma \in H\left(\operatorname{div}, \Omega ; \mathbb{R}^{3}\right), u \in L^{2}(\Omega)$ such that

$$
\begin{gathered}
\int_{\Omega} \sigma \cdot \tau \mathrm{d} x=\int_{\Omega} \operatorname{div} \tau u \mathrm{~d} x, \tau \in H\left(\operatorname{div}, \Omega ; \mathbb{R}^{3}\right), \\
\int_{\Omega} \operatorname{div} \sigma v \mathrm{~d} x=\int_{\Omega}(f-p) v \mathrm{~d} x, v \in L^{2}(\Omega), \quad \int_{\Omega} u q \mathrm{~d} x=0, q \in \mathfrak{H}^{k} .
\end{gathered}
$$

This is the standard mixed formulation for the Dirichlet problem for the Poisson equation: The first equation is equivalent to the differential equation $\sigma=-\operatorname{grad} u$ and the boundary condition $u=0$, while the second equation is equivalent to $\operatorname{div} \sigma=f$. In this case, $\mathfrak{H}^{k}=0$ so $p=0$ and the last equation is not needed. If, instead, we seek $\sigma \in H_{0}\left(\operatorname{div}, \Omega ; \mathbb{R}^{3}\right)$, then the boundary condition $u=0$ is replaced by the boundary condition $\sigma \nu=0$. Then $\mathfrak{H}^{k}=\mathbb{R}$ and so $p=\int_{\Omega} f \mathrm{~d} x / \operatorname{meas}(\Omega)$ and $\int_{\Omega} u \mathrm{~d} x=0$. These are the only boundary value problems when $k=3$. Since $\mathrm{d} u=0$, this problem is already of the form (7.4).

For $k=2$, the unknowns $\sigma \in H\left(\operatorname{curl}, \Omega ; \mathbb{R}^{3}\right)$ and $u \in H\left(\operatorname{div}, \Omega ; \mathbb{R}^{3}\right)$ satisfy the differential equations

$$
\sigma=\operatorname{curl} u, \quad \operatorname{curl} \sigma-\operatorname{grad} \operatorname{div} u=f-p,
$$

the auxiliary condition $P_{\mathfrak{H}}^{k} u=0$, and the boundary conditions $u \times \nu=0$, $\operatorname{div} u=0$ on $\partial \Omega$, so this is a mixed formulation for the vectorial Poisson equation

$$
\text { (curl curl }-\operatorname{grad} \operatorname{div}) u=f-p,
$$

with the auxiliary variable $\sigma=\operatorname{curl} u$. If instead, we seek $\sigma \in H_{0}\left(\operatorname{curl}, \Omega ; \mathbb{R}^{3}\right)$ and $u \in H_{0}\left(\operatorname{div}, \Omega ; \mathbb{R}^{3}\right)$, then we obtain the boundary conditions $\sigma \times \nu=0$ and $u \cdot \nu=0$. Finally, seeking $\sigma \in H\left(\operatorname{curl}, \Omega ; \mathbb{R}^{3}\right)$ and $u \in H_{0}\left(\operatorname{div}, \Omega ; \mathbb{R}^{3}\right)$, we obtain the boundary conditions $u \times \nu=0$ and $u \cdot n=0$. When $k=2$, (7.4) becomes

$$
\sigma_{\mathrm{d}}=\operatorname{curl} u_{\mathrm{d}}, \quad \operatorname{curl} \sigma_{\mathrm{d}}=f_{\mathrm{d}}, \quad \operatorname{div} u_{\mathrm{d}}=0,
$$

while problem (7.3) becomes

$$
-\operatorname{grad} \operatorname{div} u_{\delta}=f_{\delta}, \quad \operatorname{curl} u_{\delta}=0 .
$$

In fact, since $f_{\delta}=\operatorname{grad} F$ for some $F$, this problem has the equivalent form

$$
-\operatorname{div} u_{\delta}=F, \quad \operatorname{curl} u_{\delta}=0 .
$$


For $k=1,(7.1)$ is a different mixed formulation of the vectorial Poisson equation (7.5). Now $\sigma \in H^{1}(\Omega)$ and $u \in H\left(\operatorname{curl}, \Omega ; \mathbb{R}^{3}\right)$ satisfy the differential equations

$$
\sigma=-\operatorname{div} u, \quad \operatorname{grad} \sigma+\operatorname{curl} \operatorname{curl} u=f-p,
$$

the auxiliary condition $P_{\mathfrak{H}^{k}} u=0$, and the boundary conditions $u \cdot \nu=0$, $(\operatorname{curl} u) \times \nu=0$. If instead, we seek $\sigma \in H_{0}^{1}(\Omega)$ and $u \in H_{0}\left(\operatorname{curl}, \Omega ; \mathbb{R}^{3}\right)$, then we obtain the boundary conditions $\sigma=0$ and $u \times \nu=0$. Finally, seeking $\sigma \in H^{1}(\Omega)$ and $u \in H_{0}\left(\operatorname{curl}, \Omega ; \mathbb{R}^{3}\right)$, we obtain the boundary conditions $u \cdot \nu=0$ and $u \times \nu=0$. When $k=1$, (7.4) becomes

$$
\sigma_{\mathrm{d}}=-\operatorname{div} u_{\mathrm{d}}, \quad \operatorname{grad} \sigma_{\mathrm{d}}=f_{\mathrm{d}}, \quad \operatorname{curl} u_{\mathrm{d}}=0,
$$

while problem (7.3) becomes

$$
\text { curl curl } u_{\delta}=f_{\delta}, \quad \operatorname{div} u_{\delta}=0 .
$$

Finally, we interpret the case $k=0$. In this case $H \Lambda^{-1}(\Omega)=0$, so $\sigma=0$ and we can ignore the first equation of (7.1). Then $u \in H^{1}(\Omega)$ and $p \in \mathfrak{H}^{0}=\mathbb{R}$ satisfy

$\int_{\Omega} \operatorname{grad} u \cdot \operatorname{grad} v \mathrm{~d} x=\int_{\Omega}(f-p) v \mathrm{~d} x, v \in H^{1}(\Omega), \quad \int_{\Omega} u q \mathrm{~d} x=0, q \in \mathbb{R}$.

Thus, $p=\int_{\Omega} f \mathrm{~d} x / \operatorname{meas}(\Omega)$, and we just have the usual weak formulation of the Neumann problem for the Poisson equation $-\Delta u=f-p$. If instead, we seek $u \in H_{0}^{1}(\Omega)$, then $p=0$ and we obtain the usual weak formulation of the Dirichlet problem for Poisson's equation. For $k=0$, problem (7.4) is vacuous while problem (7.3) becomes $-\Delta u_{\delta}=f_{\delta}$.

\subsection{Well-posedness of the mixed formulation}

To discuss the well-posedness of the system (7.1), we let $B:\left[H \Lambda^{k-1}(\Omega) \times\right.$ $\left.H \Lambda^{k}(\Omega) \times \mathfrak{H}^{k}\right] \times\left[H \Lambda^{k-1}(\Omega) \times H \Lambda^{k}(\Omega) \times \mathfrak{H}^{k}\right] \rightarrow \mathbb{R}$ denote the bounded bilinear form

$$
B(\sigma, u, p ; \tau, v, q)=\langle\sigma, \tau\rangle-\langle\mathrm{d} \tau, u\rangle+\langle\mathrm{d} \sigma, v\rangle+\langle\mathrm{d} u, \mathrm{~d} v\rangle+\langle v, p\rangle-\langle u, q\rangle
$$

Well-posedness of the system (7.1) is equivalent to the inf-sup condition for $B$ (Babuška and Aziz 1972), i.e., we must establish the following result.

Theorem 7.2. There exist constants $\gamma>0, C<\infty$ such that, for any $(\sigma, u, p) \in H \Lambda^{k-1}(\Omega) \times H \Lambda^{k}(\Omega) \times \mathfrak{H}^{k}$, there exists $(\tau, v, q) \in H \Lambda^{k-1}(\Omega) \times$ $H \Lambda^{k}(\Omega) \times \mathfrak{H}^{k}$ with

$$
\begin{aligned}
B(\sigma, u, p ; \tau, v, q) & \geq \gamma\left(\|\sigma\|_{H \Lambda}^{2}+\|u\|_{H \Lambda}^{2}+\|p\|^{2}\right) \\
\|\tau\|_{H \Lambda}+\|v\|_{H \Lambda}+\|q\| & \leq C\left(\|\sigma\|_{H \Lambda}+\|u\|_{H \Lambda}+\|p\|\right)
\end{aligned}
$$


Proof. By the Hodge decomposition, given $u \in H \Lambda^{k}(\Omega)$, there exist forms $u_{\mathrm{d}} \in \mathfrak{B}^{k}, u_{\mathfrak{H}} \in \mathfrak{H}^{k}$, and $u_{\delta} \in \mathfrak{B}^{* k}$, such that

$$
u=u_{\mathrm{d}}+u_{\mathfrak{H}}+u_{\delta}, \quad\|u\|^{2}=\left\|u_{\mathrm{d}}\right\|^{2}+\left\|u_{\mathfrak{H}}\right\|^{2}+\left\|u_{\delta}\right\|^{2} .
$$

Since $u_{\mathrm{d}} \in \mathfrak{B}^{k}, u_{\mathrm{d}}=\mathrm{d} \rho$, for some $\rho \in \mathfrak{Z}^{k-1 \perp}$. Since $\mathfrak{B}^{* k}=\mathfrak{Z}^{k \perp}$ and $\mathrm{d} u_{\delta}=\mathrm{d} u$, we get using the Poincaré inequality (2.17) that

$$
\|\rho\| \leq K^{\prime}\left\|u_{\mathrm{d}}\right\|, \quad\left\|u_{\delta}\right\| \leq K\|\mathrm{~d} u\|,
$$

where $K$ and $K^{\prime}$ are constants independent of $\rho$ and $u_{\delta}$. Let $\tau=\sigma-$ $t \rho \in H \Lambda^{k-1}(\Omega), v=u+\mathrm{d} \sigma+p \in H \Lambda^{k}(\Omega)$, and $q=p-u_{\mathfrak{H}} \in \mathfrak{H}^{k}$, with $t=1 /\left(K^{\prime}\right)^{2}$. Using (7.8) and (7.9), and a simple computation, we get

$$
\begin{aligned}
& B(\sigma, u, p ; \tau, v, q) \\
& =\|\sigma\|^{2}+\|\mathrm{d} \sigma\|^{2}+\|\mathrm{d} u\|^{2}+\|p\|^{2}+t\left\|u_{\mathrm{d}}\right\|^{2}+\left\|u_{\mathfrak{H}}\right\|^{2}-t\langle\sigma, \rho\rangle \\
& \geq \frac{1}{2}\|\sigma\|^{2}+\|\mathrm{d} \sigma\|^{2}+\|\mathrm{d} u\|^{2}+\|p\|^{2}+t\left\|u_{\mathrm{d}}\right\|^{2}+\left\|u_{\mathfrak{H}}\right\|^{2}-\frac{t^{2}}{2}\|\rho\|^{2} \\
& \geq \frac{1}{2}\|\sigma\|^{2}+\|\mathrm{d} \sigma\|^{2}+\|\mathrm{d} u\|^{2}+\|p\|^{2}+\left\|u_{\mathfrak{H}}\right\|^{2}+\left\|u_{\mathrm{d}}\right\|^{2}\left(t-t^{2}\left(K^{\prime}\right)^{2} / 2\right) \\
& \geq \frac{1}{2}\|\sigma\|^{2}+\|\mathrm{d} \sigma\|^{2}+\frac{1}{2}\|\mathrm{~d} u\|^{2}+\|p\|^{2}+\left\|u_{\mathfrak{H}}\right\|^{2}+\frac{1}{2\left(K^{\prime}\right)^{2}}\left\|u_{\mathrm{d}}\right\|^{2}+\frac{1}{2 K^{2}}\left\|u_{\delta}\right\|^{2} \\
& \geq \frac{1}{2}\|\sigma\|^{2}+\|\mathrm{d} \sigma\|^{2}+\frac{1}{2}\|\mathrm{~d} u\|^{2}+\frac{1}{2\left(K^{\prime \prime}\right)^{2}}\|u\|^{2}+\|p\|^{2},
\end{aligned}
$$

where $K^{\prime \prime}=\max \left(K^{\prime}, K, 1 / \sqrt{2}\right)$. Hence, we obtain (7.6) with $\gamma>0$ depending only on $K$ and $K^{\prime}$. The upper bound (7.7) follows easily from (7.8) and (7.9).

Remark. If, instead, we consider the form $B$ over the space $\left[\stackrel{\circ}{H} \Lambda^{k-1}(\Omega) \times\right.$ $\left.\stackrel{\circ}{H} \Lambda^{k}(\Omega) \times \stackrel{\circ}{\mathfrak{H}}^{k}\right] \times\left[\stackrel{\circ}{H} \Lambda^{k-1}(\Omega) \times \stackrel{\circ}{H} \Lambda^{k}(\Omega) \times \stackrel{\mathfrak{H}}{\mathfrak{H}}^{k}\right]$ then the stability result is still valid. The proof must be modified to use the Hodge decomposition $u=u_{\mathrm{d}}+u_{\mathfrak{H}}+u_{\delta}$, where now $u_{\mathrm{d}} \in \stackrel{\circ}{\mathfrak{B}}^{k}, u_{\mathfrak{H}} \in \stackrel{\mathfrak{H}}{ }^{k}$, and $u_{\delta} \in \mathfrak{B}^{* k}$.

\subsection{Well-posedness of discretizations of the mixed formulation}

We next consider discrete versions of these results. Suppose we are given a triangulation, and let

$$
0 \rightarrow \Lambda_{h}^{0} \stackrel{\mathrm{d}}{\rightarrow} \Lambda_{h}^{1} \stackrel{\mathrm{d}}{\rightarrow} \cdots \stackrel{\mathrm{d}}{\rightarrow} \Lambda_{h}^{n} \rightarrow 0
$$

denote any of the $2^{n-1}$ finite element de Rham complexes (for each value of the degree) discussed previously. Recall we have a commuting diagram of 
the form

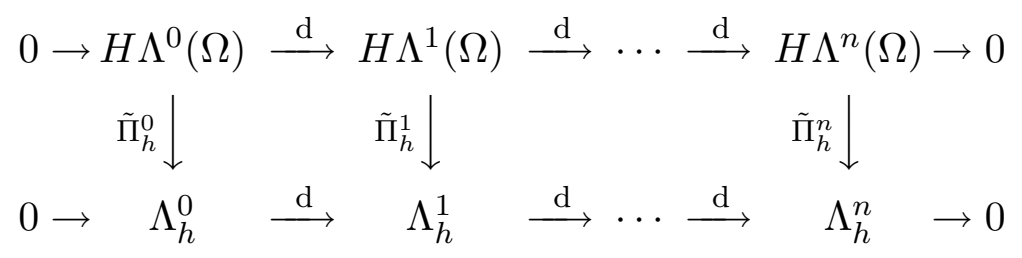

where the $\tilde{\Pi}_{h}^{k}$ are bounded projections, i.e.,

$$
\left\|\tilde{\Pi}_{h}^{k} \omega\right\|_{H \Lambda^{k}} \leq C\|\omega\|_{H \Lambda^{k}}, \quad \omega \in H \Lambda^{k}(\Omega),
$$

with the constant $C$ independent of $\omega$ and $h$. We note that the canonical interpolation operators associated to the standard finite element spaces do not satisfy these conditions, since their definition requires more regularity. However, the new projection operators discussed in Section 5 do satisfy these conditions, so we can assume we have such projection operators. Of course, we would also like the results presented below to apply to problems with essential boundary conditions. In that case, we would need projection operators $\tilde{\Pi}_{h}^{k}$ mapping $\stackrel{\circ}{H} \Lambda^{k}$ to $\AA_{h}^{k}$ that again satisfy (7.12). Although our construction in Section 5 did not include this case, we believe that such a construction is also possible, and we shall assume that we have such projection operators in this case also.

Under these conditions, we shall next demonstrate stability of the finite element method: find $\sigma_{h} \in \Lambda_{h}^{k-1}, u_{h} \in \Lambda_{h}^{k}, p_{h} \in \mathfrak{H}_{h}^{k}$ such that

$$
\begin{aligned}
\left\langle\sigma_{h}, \tau\right\rangle & =\left\langle\mathrm{d} \tau, u_{h}\right\rangle, & & \tau \in \Lambda_{h}^{k-1}, \\
\left\langle\mathrm{~d} \sigma_{h}, v\right\rangle+\left\langle\mathrm{d} u_{h}, \mathrm{~d} v\right\rangle+\langle v, p\rangle & =\langle f, v\rangle, & & v \in \Lambda_{h}^{k}, \\
\langle u, q\rangle & =0, & & q \in \mathfrak{H}_{h}^{k} .
\end{aligned}
$$

In view of the discrete de Rham complexes obtained in Section 5.5, this result will apply to prove stability of four different families of mixed methods for the Hodge Laplacian problem, using any of the four choices of spaces

$$
\begin{array}{rlrl}
\mathcal{P}_{r}^{-} \Lambda^{k-1}\left(\mathcal{T}_{h}\right) & \times \mathcal{P}_{r}^{-} \Lambda^{k}\left(\mathcal{T}_{h}\right), & \mathcal{P}_{r} \Lambda^{k-1}\left(\mathcal{T}_{h}\right) & \times \mathcal{P}_{r}^{-} \Lambda^{k}\left(\mathcal{T}_{h}\right), \\
\mathcal{P}_{r+1}^{-} \Lambda^{k-1}\left(\mathcal{T}_{h}\right) & \times \mathcal{P}_{r} \Lambda^{k}\left(\mathcal{T}_{h}\right), & \mathcal{P}_{r+1} \Lambda^{k-1}\left(\mathcal{T}_{h}\right) \times \mathcal{P}_{r} \Lambda^{k}\left(\mathcal{T}_{h}\right),
\end{array}
$$

to discretize the $(k-1)$-forms and the $k$-forms, respectively. Since the $(k-1)$-forms disappear for $k=0$, and since we have $\mathcal{P}_{r}^{-} \Lambda^{0}\left(\mathcal{T}_{h}\right)=\mathcal{P}_{r} \Lambda^{0}\left(\mathcal{T}_{h}\right)$ and $\mathcal{P}_{r}^{-} \Lambda^{n}\left(\mathcal{T}_{h}\right)=\mathcal{P}_{r-1} \Lambda^{n}\left(\mathcal{T}_{h}\right)$, these reduce to a single family of methods for $k=0$ (namely, the use of the standard Lagrange elements for the standard Laplacian problem), and to two families of methods for $k=1$ or $k=n$.

Stability of the method (7.13) is equivalent to the inf-sup condition for $B$ restricted to the finite element spaces (Babuška and Aziz 1972), i.e., we must establish the following result.

Theorem 7.3. There exist constants $\gamma>0, C<\infty$ independent of $h$ such 
that for any $(\sigma, u, p) \in \Lambda_{h}^{k-1} \times \Lambda_{h}^{k} \times \mathfrak{H}_{h}^{k}$, there exists $(\tau, v, q) \in \Lambda_{h}^{k-1} \times \Lambda_{h}^{k} \times \mathfrak{H}_{h}^{k}$ with

$$
\begin{aligned}
B(\sigma, u, p ; \tau, v, q) & \geq \gamma\left(\|\sigma\|_{H \Lambda}^{2}+\|u\|_{H \Lambda}^{2}+\|p\|^{2}\right), \\
\|\tau\|_{H \Lambda}+\|v\|_{H \Lambda}+\|q\| & \leq C\left(\|\sigma\|_{H \Lambda}+\|u\|_{H \Lambda}+\|p\|\right) .
\end{aligned}
$$

Proof. The proof in the discrete case follows closely the proof given above for the continuous case. By the discrete Hodge decomposition, given $u \in \Lambda_{h}^{k}$, there exist forms $u_{\mathrm{d}} \in \mathfrak{B}_{h}^{k}, u_{\mathfrak{H}} \in \mathfrak{H}_{h}^{k}$, and $u_{\delta} \in \mathfrak{Z}_{h}^{k \perp}$, such that

$$
u=u_{\mathrm{d}}+u_{\mathfrak{H}}+u_{\delta}, \quad\|u\|^{2}=\left\|u_{\mathrm{d}}\right\|^{2}+\left\|u_{\mathfrak{H}}\right\|^{2}+\left\|u_{\delta}\right\|^{2} .
$$

Since $u_{\mathrm{d}} \in \mathfrak{B}_{h}^{k}, u_{\mathrm{d}}=\mathrm{d} \rho$, for some $\rho \in \mathfrak{Z}_{h}^{k-1 \perp}$. Since $\mathrm{d} u_{\delta}=\mathrm{d} u$, we get using the discrete Poincaré inequality, Theorem 5.11, that

$$
\|\rho\| \leq K^{\prime}\left\|u_{\mathrm{d}}\right\|, \quad\left\|u_{\delta}\right\| \leq K\|\mathrm{~d} u\|,
$$

where $K$ and $K^{\prime}$ are constants independent of $\rho, u_{\delta}$, and $h$. The result now follows by applying the same proof as in the continuous case, where we use (7.15) and (7.16) in place of (7.8) and (7.9). To handle other boundary conditions, the discrete Hodge decomposition must be modified as in the continuous case.

From this stability result, we then obtain the following quasioptimal error estimates.

Theorem 7.4. Let $(\sigma, u, p) \in H \Lambda^{k-1}(\Omega) \times H \Lambda^{k}(\Omega) \times \mathfrak{H}^{k}$ be the solution of problem (7.1) and $\left(\sigma_{h}, u_{h}, p_{h}\right) \in \Lambda_{h}^{k-1} \times \Lambda_{h}^{k} \times \mathfrak{H}_{h}^{k}$ the solution of problem (7.13). Then

$$
\begin{aligned}
& \left\|\sigma-\sigma_{h}\right\|_{H \Lambda}+\left\|u-u_{h}\right\|_{H \Lambda}+\left\|p-p_{h}\right\| \\
& \quad \leq C\left(\inf _{\tau \in \Lambda_{h}^{k-1}}\|\sigma-\tau\|_{H \Lambda}+\inf _{v \in \Lambda_{h}^{k}}\|u-v\|_{H \Lambda}+\inf _{q \in \mathfrak{H}_{h}^{k}}\|p-q\|+\left\|P_{\mathfrak{H}_{h}^{k}} u\right\|\right),
\end{aligned}
$$

where $P_{\mathfrak{H}_{h}^{k}} u$ denotes the $L^{2}$-projection of $u$ into $\mathfrak{H}_{h}^{k}$. Moreover,

$$
\left\|P_{\mathfrak{H}_{h}^{k}} u\right\| \leq \inf _{r \in \mathfrak{H}^{k}}\|q-r\| \inf _{v_{\mathrm{d}} \in \mathfrak{B}_{h}^{k}}\left\|u_{\mathrm{d}}-v_{\mathrm{d}}\right\| \leq \varepsilon_{h} \inf _{v_{\mathrm{d}} \in \mathfrak{B}_{h}^{k}}\left\|u_{\mathrm{d}}-v_{\mathrm{d}}\right\|,
$$

where $u_{\mathrm{d}}$ is the $L^{2}$-projection of $u$ into $\mathfrak{B}^{k}, q=P_{\mathfrak{H}_{h}^{k}} u /\left\|P_{\mathfrak{H}_{h}^{k}} u\right\|$ and

$$
\varepsilon_{h}=\sup _{\substack{r \in \mathfrak{H}^{k} \\\|r\|=1}}\left\|\left(I-\tilde{\Pi}_{h}\right) r\right\| .
$$

Proof. First observe that $(\sigma, u, p)$ satsifies

$$
B\left(\sigma, u, p ; \tau_{h}, v_{h}, q_{h}\right)=\left\langle f, v_{h}\right\rangle-\left\langle u, q_{h}\right\rangle, \quad\left(\tau_{h}, v_{h}, q_{h}\right) \in \Lambda_{h}^{k-1} \times \Lambda_{h}^{k} \times \mathfrak{H}_{h}^{k} .
$$


Let $\tau \in \Lambda_{h}^{k-1}, u \in \Lambda_{h}^{k}, q \in \mathfrak{H}_{h}^{k}$. Then, for any $\left(\tau_{h}, v_{h}, q_{h}\right) \in \Lambda_{h}^{k-1} \times \Lambda_{h}^{k} \times \mathfrak{H}_{h}^{k}$, we have

$$
\begin{aligned}
B\left(\sigma_{h}-\right. & \left.\tau, u_{h}-v, p_{h}-q ; \tau_{h}, v_{h}, q_{h}\right) \\
= & B\left(\sigma-\tau, u-v, p-q ; \tau_{h}, v_{h}, q_{h}\right)+\left\langle u, q_{h}\right\rangle \\
= & B\left(\sigma-\tau, u-v, p-q ; \tau_{h}, v_{h}, q_{h}\right)+\left\langle P_{\mathfrak{H}_{h}^{k}} u, q_{h}\right\rangle \\
\leq & C\left(\|\sigma-\tau\|_{H \Lambda}+\|u-v\|_{H \Lambda}+\|p-q\|+\left\|P_{\mathfrak{H}_{h}^{k}} u\right\|\right) \\
& \quad \times\left(\left\|\tau_{h}\right\|_{H \Lambda}+\left\|v_{h}\right\|_{H \Lambda}+\left\|q_{h}\right\|\right) .
\end{aligned}
$$

Theorem 7.3 then gives

$$
\begin{aligned}
\left\|\sigma_{h}-\tau\right\|_{H \Lambda}+\| u_{h}- & v\left\|_{H \Lambda}+\right\| p_{h}-q \| \\
& \leq C\left(\|\sigma-\tau\|_{H \Lambda}+\|u-v\|_{H \Lambda}+\|p-q\|+\left\|P_{\mathfrak{H}_{h}^{k}} u\right\|\right),
\end{aligned}
$$

from which (7.17) follows by the triangle inequality.

Now $u \perp \mathfrak{H}^{k}$, so $u=u_{\mathrm{d}}+u_{\delta}$, with $u_{\mathrm{d}} \in \mathfrak{B}^{k}$ and $u_{\delta} \in \mathfrak{Z}^{k \perp}$. Since $\mathfrak{H}_{h}^{k} \subset \mathfrak{Z}^{k}$, $P_{\mathfrak{H}_{h}^{k}} u_{\delta}=0$, while, by the discrete Hodge decomposition, $P_{\mathfrak{H}_{h}^{k}} v_{\mathrm{d}}=0$ for all $v_{\mathrm{d}} \in \mathfrak{B}_{h}^{k}$. Let $q=P_{\mathfrak{H}_{h}^{k}} u /\left\|P_{\mathfrak{H}_{h}^{k}} u\right\| \in \mathfrak{H}_{h}^{k}$. For any $v_{\mathrm{d}} \in \mathfrak{B}_{h}^{k}$ we have

$$
\begin{aligned}
\left\|P_{\mathfrak{H}_{h}^{k}} u\right\|=\left\langle u_{\mathrm{d}}-v_{\mathrm{d}}, q\right\rangle & =\inf _{r \in \mathfrak{H}^{k}}\left\langle u_{\mathrm{d}}-v_{\mathrm{d}}, q-r\right\rangle \\
& \leq\left\|u_{\mathrm{d}}-v_{\mathrm{d}}\right\| \inf _{r \in \mathfrak{H}^{k}}\|q-r\| .
\end{aligned}
$$

Furthermore, by Lemma 5.9, we can find $r \in \mathfrak{H}^{k}$ with $\|r\| \leq 1$ and $\|q-r\| \leq$ $\left\|\left(I-\tilde{\Pi}_{h}\right) r\right\| \leq \varepsilon_{h}$, and hence (7.18) follows.

Remark. Let $u$ be as in the theorem above. Since $u \perp \mathfrak{H}^{k}$, it follows that if $\mathfrak{H}_{h}^{k} \subset \mathfrak{H}^{k}$, then $P_{\mathfrak{H}_{h}^{k}} u=0$. On the other hand, if $\Lambda_{h}^{k-1} \times \Lambda_{h}^{k}$ is one of the choices given in (7.14) and $u_{d}$ and all elements of $\mathfrak{H}^{k}$ are sufficiently smooth, then

$$
\left\|P_{\mathfrak{H}_{h}^{k}} u\right\| \leq \varepsilon_{h}\left\|\left(I-\tilde{\Pi}_{h}\right) u_{d}\right\|=\mathcal{O}\left(h^{2 r}\right)
$$

If the solution $(\sigma, u, p)$ is sufficiently smooth, we then obtain the following order of convergence estimates.

Corollary 7.5. If $\Lambda_{h}^{k-1} \times \Lambda_{h}^{k}$ is one of the choices given in (7.14) and $\left\|P_{\mathfrak{H}_{h}^{k}} u\right\|=\mathcal{O}\left(h^{r}\right)$, then

$$
\left\|\sigma-\sigma_{h}\right\|_{H \Lambda}+\left\|u-u_{h}\right\|_{H \Lambda}+\left\|p-p_{h}\right\|=\mathcal{O}\left(h^{r}\right) .
$$

Proof. This result follows from the previous theorem by using the approximation properties of the subspaces given in Theorems 5.6 and 5.8.

Remark. As noted earlier, problems (7.3) and (7.4) are special cases of 
problem (7.1) when $f=f_{\delta} \in \mathfrak{B}^{* k}$ or $f=f_{\mathrm{d}} \in \mathfrak{B}^{k}$. Although these reduced problems have a simpler form, they are not so easy to approximate directly by finite element methods, since that would involve finding a basis for finite element subspaces of $\mathfrak{B}^{k}$ or $\mathfrak{B}^{* k}$. However, since they are equivalent to problem (7.1), one can use the discretization of problem (7.1) with standard finite element spaces to find good approximations to problems of this type.

Remark. We also note that an early use of a discrete Hodge decomposition and discrete Poincaré inequality to establish stability of mixed finite element methods appears in the work of Fix, Gunzburger and Nicolaides (1981), in connection with the grid decomposition principle. See also Bochev and Gunzburger (2005) for a more recent exposition.

\subsection{Improved error estimates - basic bounds}

As is well known from the theory of mixed finite element methods (Falk and Osborn 1980, Douglas and Roberts 1985), it is sometimes possible to get improved error estimates for each term in the mixed formulation by decoupling them. In this subsection, we show how this more refined analysis can be carried out for the mixed finite element approximation of the Hodge Laplacian. In particular, we show that for any polyhedral domain $\Omega$ and any $f \in L^{2} \Lambda^{k}(\Omega)$, we have

$$
\left\|\sigma-\sigma_{h}\right\|+\left\|u-u_{h}\right\|_{H \Lambda}+\left\|p-p_{h}\right\|=\mathcal{O}\left(h^{1 / 2}\right) .
$$

Without the assumption of additional regularity on $f$, such an estimate can not be obtained from the quasioptimal result stated above, since the error in that estimate also depends on the approximation of $\mathrm{d} \sigma=f_{\mathrm{d}}$ and this requires more regularity than just $f_{\mathrm{d}} \in L^{2} \Lambda^{k}(\Omega)$ to achieve a positive rate of convergence. Higher order improved rates of convergence with less regularity can also be obtained by using this more refined analysis.

To obtain improved error estimates, we introduce $P_{h}: L^{2} \Lambda^{k}(\Omega) \rightarrow \mathfrak{B}_{h}^{k} \oplus$ $\mathfrak{H}^{k} \oplus Z^{k \perp}$ as the $L^{2}$-projection, i.e., $P_{h}$ is the identity on $\mathfrak{H}^{k} \oplus Z^{k \perp}$, but is the $L^{2}$-projection onto $\mathfrak{B}_{h}^{k}$ on $\mathfrak{B}^{k}$. We then break up the solutions of problems (7.1) and (7.13) into two pieces, where the right-hand side $f$ is replaced by either $\left(I-P_{h}\right) f$ or $P_{h} f$. Our error analysis will be based on a separate analysis for each of these pieces Note that the first problem represents a perturbation of the right-hand side and we will estimate how both the true and approximate solutions depend on this perturbation. In the second problem, we have made a slight modification to the right-hand side, and we will estimate the usual error between the true and approximate solutions. These results are contained in the three lemmas that follow. Note that the estimates in this subsection make no assumptions about the regularity of the solution or the approximation properties of the finite element subspaces. 
Although we write the results using the operators $\tilde{\Pi}_{h}$, we only assume in this subsection that $\tilde{\Pi}_{h}=\tilde{\Pi}_{h}^{k}$ is a projection operator mapping into $\Lambda_{h}^{k}$ that satisfies $\mathrm{d} \tilde{\Pi}_{h}^{k-1} \rho=\tilde{\Pi}_{h}^{k} \mathrm{~d} \rho$ for all sufficiently smooth $\rho \in H \Lambda^{k-1}$.

Lemma 7.6. Let $(\sigma, u, p) \in H \Lambda^{k-1}(\Omega) \times H \Lambda^{k}(\Omega) \times \mathfrak{H}^{k}$ be the solution of problem (7.1) with right-hand side $f$ replaced by $\left(I-P_{h}\right) f$. Then $u \in \mathfrak{B}^{k}$, $p=0$, and $\mathrm{d} \sigma=\left(I-P_{h}\right) f$. Furthermore,

$$
\|u\|^{2}+\|\sigma\|^{2} \leq C\left\|\left(I-P_{h}\right) f\right\|\left\|\left(I-P_{h}\right) u\right\| .
$$

Proof. Since the right-hand side $\left(I-P_{h}\right) f \in \mathfrak{B}^{k}$ we can conclude that $u \in \mathfrak{B}^{k}, p=0$, and $\mathrm{d} \sigma=\left(I-P_{h}\right) f$. Choosing $\tau=\sigma$ in the first equation of the system (7.1), we get

$$
\begin{aligned}
\|\sigma\|^{2}=\langle\mathrm{d} \sigma, u\rangle= & \left\langle\left(I-P_{h}\right) f, u\right\rangle \\
& =\left\langle\left(I-P_{h}\right) f,\left(I-P_{h}\right) u\right\rangle \leq\left\|\left(I-P_{h}\right) f\right\|\left\|\left(I-P_{h}\right) u\right\| .
\end{aligned}
$$

Next, since $u \in \mathfrak{B}^{k}$, there exists $\tau \in \mathfrak{Z}^{k-1 \perp}$, with $\mathrm{d} \tau=u$. Then, from the first equation of the system (7.1) with this choice of $\tau$ and Poincaré's inequality, we get

$$
\|u\|^{2}=(\mathrm{d} \tau, u)=(\sigma, \tau) \leq\|\sigma\|\|\tau\| \leq C\|\sigma\|\|\mathrm{d} \tau\| \leq C\|\sigma\|\|u\| .
$$

Hence, $\|u\| \leq C\|\sigma\|$, and the theorem follows immediately.

The properties of the solution of the discrete problem (7.13) with right hand side $\left(I-P_{h}\right) f$ are strikingly different from the continuous case.

Lemma 7.7. Let $\left(\sigma_{h}, u_{h}, p_{h}\right) \in \Lambda_{h}^{k-1} \times \Lambda_{h}^{k} \times \mathfrak{H}_{h}^{k}$ be the finite element solution of problem (7.13) with right-hand side $f$ replaced by $\left(I-P_{h}\right) f$. Then $\sigma_{h}=0, u_{h} \in \mathfrak{B}_{h}^{k \perp}$, and the solution satisfies the estimates

$$
\begin{gathered}
\left\|u_{h}\right\|_{H \Lambda}^{2} \leq C\left\|\left(I-P_{h}\right) f\right\|\left\|\left(I-\tilde{\Pi}_{h}\right) w^{h}\right\|, \\
\left\|p_{h}\right\|^{2} \leq\left\|\left(I-P_{h}\right) f\right\|\left\|\left(I-\tilde{\Pi}_{h}\right) r\right\|,
\end{gathered}
$$

where $w^{h} \in \mathfrak{Z}^{k \perp}$ satisfies $\mathrm{d} w^{h}=\mathrm{d} u_{h}$, and $r \in \mathfrak{H}^{k}$ satisfies $\|r\| \leq\left\|\left(I-P_{h}\right) f\right\|$.

Proof. Since the right-hand side $\left(I-P_{h}\right) f$ is $L^{2}$-orthogonal to $\mathfrak{B}_{h}^{k}$, it follows that $\sigma_{h}=0$ and $u_{h} \in \mathfrak{Z}_{h}^{k \perp}$. Furthermore,

$$
\left\langle\mathrm{d} u_{h}, \mathrm{~d} v\right\rangle+\left\langle p_{h}, v\right\rangle=\left\langle\left(I-P_{h}\right) f, v\right\rangle, \quad v \in \Lambda_{h}^{k} .
$$

The estimate on $\mathrm{d} u_{h}$ follows by choosing $v=u_{h}$ and using the discrete Poincaré inequality. Defining $w \in \mathfrak{Z}^{k \perp}$ satisfying $\mathrm{d} w=\mathrm{d} u_{h}$, we get, by Lemma 5.10, that

$$
\begin{aligned}
\left\|\mathrm{d} u_{h}\right\|^{2}=\left\langle\left(I-P_{h}\right) f, u_{h}\right\rangle & =\left\langle\left(I-P_{h}\right) f, u_{h}-w\right\rangle \\
& \leq\left\|\left(I-P_{h}\right) f\right\|\left\|w-\tilde{\Pi}_{h} w\right\| .
\end{aligned}
$$


The first estimate of the lemma now follows from the discrete Poincaré inequality. Note also that (7.19) implies that $\left\|p_{h}\right\| \leq\left\|\left(I-P_{h}\right) f\right\|$. Therefore, using Lemma 5.9, there is an harmonic $k$-form $r \in \mathfrak{H}^{k}$ such that $\|r\| \leq$ $\left\|\left(I-P_{h}\right) f\right\|$ and $\left\|p_{h}-r\right\| \leq\left\|\left(I-\tilde{\Pi}_{h}\right) r\right\|$. By applying (7.19) once more, we obtain

$$
\left\|p_{h}\right\|^{2}=\left\langle\left(I-P_{h}\right) f, p_{h}\right\rangle=\left\langle\left(I-P_{h}\right) f, p_{h}-r\right\rangle,
$$

and hence the final estimate follows from Cauchy-Schwarz inequality.

Finally, we compare the solutions of the problems (7.1) and (7.13) when the right-hand side is of the form $P_{h} f$.

Lemma 7.8. Let $(\sigma, u, p) \in H \Lambda^{k-1}(\Omega) \times H \Lambda^{k}(\Omega) \times \mathfrak{H}^{k}$ be the solution of problem (7.1) with right-hand side $f$ replaced by $P_{h} f$, and $\left(\sigma_{h}, u_{h}, p_{h}\right) \in$ $\Lambda_{h}^{k-1} \times \Lambda_{h}^{k} \times \mathfrak{H}_{h}^{k}$ be the corresponding finite element solution of problem (7.13). Then $\mathrm{d} \sigma_{h}=\mathrm{d} \sigma$ and

$$
\left\|\sigma-\sigma_{h}\right\| \leq\left\|\sigma-\tilde{\Pi}_{h} \sigma\right\|, \quad \text { and } \quad\left\|p-p_{h}\right\| \leq\left\|\left(I-\tilde{\Pi}_{h}\right) p\right\| .
$$

Furthermore, there is a constant $C$ independent of $h$ such that

$$
\left\|u-u_{h}\right\|_{H \Lambda} \leq C\left(\left\|u-\tilde{\Pi}_{h} u\right\|_{H \Lambda}+\left\|\sigma-\tilde{\Pi}_{h} \sigma\right\|+\left\|p-\tilde{\Pi}_{h} p\right\|+\left\|P_{\mathfrak{H}_{h}^{k}} u\right\|\right) .
$$

The term $\left\|P_{\mathfrak{H}_{h}^{k}} u\right\|$ can be estimated by as in Theorem 7.4.

Proof. Let $P_{h} f=f_{\mathrm{d}}+f_{\mathfrak{H}}+f_{\delta}$ be the Hodge decomposition of $P_{h} f$. Then $f_{\mathrm{d}} \in \mathfrak{B}_{h}^{k} \subset \mathfrak{B}^{k}$. As a consequence, $\mathrm{d} \sigma=\mathrm{d} \sigma_{h}=f_{\mathrm{d}}$. Hence $\mathrm{d}\left(\tilde{\Pi}_{h} \sigma-\sigma_{h}\right)=$ $\tilde{\Pi}_{h} \mathrm{~d} \sigma-\mathrm{d} \sigma_{h}=0$. Then from the first error equation, we have

$$
\begin{aligned}
\left\|\sigma-\sigma_{h}\right\|^{2} & =\left\langle\sigma-\sigma_{h}, \sigma-\tilde{\Pi}_{h} \sigma\right\rangle+\left\langle\mathrm{d}\left(\tilde{\Pi}_{h} \sigma-\sigma_{h}\right), u-u_{h}\right\rangle \\
& =\left\langle\sigma-\sigma_{h}, \sigma-\tilde{\Pi}_{h} \sigma\right\rangle .
\end{aligned}
$$

Hence,

$$
\left\|\sigma-\sigma_{h}\right\| \leq\left\|\sigma-\tilde{\Pi}_{h} \sigma\right\| .
$$

Since $\mathrm{d}\left(\sigma-\sigma_{h}\right)=0$ we also obtain the error equation

$$
\left\langle\mathrm{d}\left(u-u_{h}\right), \mathrm{d} v\right\rangle+\left\langle p-p_{h}, v\right\rangle=0, \quad v \in \Lambda_{h}^{k} .
$$

In particular, this implies that $p_{h}$ is the $L^{2}$-projection of $p$ into $\mathfrak{H}_{h}^{k}$, and therefore, using Lemma 5.8, we obtain

$$
\left\|p-p_{h}\right\| \leq\left\|\left(I-\tilde{\Pi}_{h}\right) p\right\|,
$$

which is the desired error estimate for the harmonic forms.

In order to establish the estimate for $u-u_{h}$, we decompose this error into

$$
u-u_{h}=\left(I-\tilde{\Pi}_{h}\right) u+e_{h},
$$

where $e_{h}=\tilde{\Pi}_{h} u-u_{h} \in \Lambda_{h}^{k}$. We will estimate $\left\|e_{h}\right\|_{H \Lambda}$ by introducing the 
discrete Hodge decomposition

$$
e_{h}=e_{\mathrm{d}, h}+e_{\mathfrak{H}, h}+e_{\delta, h} .
$$

Note that

$$
e_{\mathfrak{H}, h}=P_{\mathfrak{H}_{h}^{k}}\left(\tilde{\Pi}_{h} u\right)=P_{\mathfrak{H}_{h}^{k}} u-P_{\mathfrak{H}_{h}^{k}}\left(I-\tilde{\Pi}_{h}\right) u
$$

and therefore

$$
\left\|e_{\mathfrak{H}, h}\right\| \leq\left\|P_{\mathfrak{H}_{h}^{k}} u\right\|+\left\|\left(I-\tilde{\Pi}_{h}\right) u\right\|
$$

Note that the solutions $(\sigma, u)$ and $\left(\sigma_{h}, u_{h}\right)$ are related by the error relation

$$
\left\langle\sigma-\sigma_{h}, \tau\right\rangle=\left\langle\mathrm{d} \tau, e_{\mathrm{d}, h}\right\rangle+\left\langle\mathrm{d} \tau,\left(I-\tilde{\Pi}_{h}\right) u\right\rangle, \quad \tau \in \Lambda_{h}^{k-1} .
$$

By picking $\tau \in Z_{h}^{(k-1) \perp}$ such that $\mathrm{d} \tau=e_{\mathrm{d}, h}$ we obtain

$$
\left\|e_{\mathrm{d}, h}\right\| \leq\left\|\left(I-\tilde{\Pi}_{h}\right) u\right\|+C\left\|\sigma-\sigma_{h}\right\|
$$

Finally, in order to estimate $\left\|e_{\delta, h}\right\|_{H \Lambda}$ we note that

$$
\left\langle\mathrm{d}\left(e_{\delta, h}, \mathrm{~d} v\right\rangle+\left\langle p-p_{h}, v\right\rangle=-\left\langle\left(I-\tilde{\Pi}_{h}\right) \mathrm{d} u, \mathrm{~d} v\right\rangle .\right.
$$

Using the discrete Poincaré inequality we then get

$$
\left\|\mathrm{d} e_{\delta, h}\right\| \leq\left\|\left(I-\tilde{\Pi}_{h}\right) \mathrm{d} u\right\|+C\left\|p-p_{h}\right\| .
$$

By (7.20), (7.21), (7.22), (7.23), and by using the discrete Poincaré inequality once more, the desired estimate for $\left\|u-u_{h}\right\|_{H \Lambda}$ follows.

In Section 7.9 we will apply this results to show that for any polyhedral domain $\Omega$ and any $f \in L^{2} \Lambda^{k}(\Omega)$, we have

$$
\left\|\sigma-\sigma_{h}\right\|+\left\|u-u_{h}\right\|_{H \Lambda}+\left\|p-p_{h}\right\|=\mathcal{O}\left(h^{1 / 2}\right)
$$

while, in case we have sufficient smoothness (but less than needed using $(7.17)$ ), we have

$$
\left\|\sigma-\sigma_{h}\right\|+\left\|u-u_{h}\right\|_{H \Lambda}+\left\|p-p_{h}\right\|=\mathcal{O}\left(h^{r}\right),
$$

\subsection{Regularity properties}

To obtain order of convergence estimates in the next subsection, we will need to make some assumption about the domain $\Omega$ that will ensure that the solution of problem (7.1) has some regularity beyond merely belonging to the space in which we seek the solution.

We shall say that the domain $\Omega$ is s-regular if, for $w \in H \Lambda^{k}(\Omega) \cap \stackrel{\circ}{H}^{*} \Lambda^{k}(\Omega)$ or $\stackrel{\circ}{H} \Lambda^{k}(\Omega) \cap H^{*} \Lambda^{k}(\Omega), w \in H^{s} \Lambda^{k}(\Omega)$ and

$$
\|w\|_{s} \leq C(\|w\|+\|\mathrm{d} w\|+\|\delta w\|)
$$

for some $0<s \leq 1$. We note for a Lipschitz domain, this result for $s=1 / 2$ 
can be found in Mitrea, Mitrea and Taylor (2001) (Theorem 11.2). When $\Omega$ is convex, this result holds for $s=1$ (a consequence of Corollary 5.2 in Mitrea (2001)) and the term $\|w\|$ may be omitted. When $\Omega$ is a polyhedron in $\mathbb{R}^{3}$, the result holds for some $1 / 2<s \leq 1$ (see Amrouche, Bernardi, Dauge and Girault (1998), Costabel (1991)). Also note that by Poincaré's inequality (Theorem 2.2), for $w \in H \Lambda^{k}(\Omega) \cap \stackrel{\circ}{H}^{*} \Lambda^{k}(\Omega) \cap \mathfrak{H}^{k \perp}$ or $\stackrel{\circ}{H} \Lambda^{k}(\Omega) \cap$ $H^{*} \Lambda^{k}(\Omega) \cap \dot{\mathfrak{H}}^{k \perp}$, we may also omit the term $\|w\|$.

\subsection{Order of convergence estimates}

Using the approximation properties of the operators $\tilde{\Pi}_{h}$ and the regularity estimate (7.24), we are able to obtain the following error estimates.

Theorem 7.9. Suppose that the domain $\Omega$ is $s-$ regular for some $0<$ $s \leq 1$. Let $(\sigma, u, p) \in H \Lambda^{k-1}(\Omega) \times H \Lambda^{k}(\Omega) \times \mathfrak{H}^{k}$ be the solution of problem $(7.1)$ and $\left(\sigma_{h}, u_{h}, p_{h}\right) \in \Lambda_{h}^{k-1} \times \Lambda_{h}^{k} \times \mathfrak{H}_{h}^{k}$ the solution of problem (7.13), where $\Lambda_{h}^{k-1} \times \Lambda_{h}^{k}$ is one of the choices given in (7.14). Then for $f \in L^{2} \Lambda^{k}(\Omega)$,

$$
\left\|\sigma-\sigma_{h}\right\|+\left\|u-u_{h}\right\|_{H \Lambda}+\left\|p-p_{h}\right\| \leq C h^{s}\|f\| .
$$

If $f_{\mathrm{d}}$ and $u_{d} \in H^{t-1} \Lambda^{k}(\Omega), \sigma \in H^{t+s-1} \Lambda^{k-1}(\Omega), u$ and $p \in H^{t+s-1} \Lambda^{k}(\Omega)$, and $\mathrm{d} u \in H^{t+s-1} \Lambda^{k+1}(\Omega)$ for $1 \leq t \leq r$, then

$$
\begin{aligned}
&\left\|\sigma-\sigma_{h}\right\| \leq C h^{t+s-1}\left(\left\|f_{\mathrm{d}}\right\|_{t-1}+\|\sigma\|_{t+s-1}\right) \\
&\left\|u-u_{h}\right\|_{H \Lambda} \leq C h^{t+s-1}\left(\left\|f_{\mathrm{d}}\right\|_{t-1}+\|\sigma\|_{t+s-1}+\left\|u_{\mathrm{d}}\right\|_{t-1}\right.\left.\quad\|u\|_{t+s-1}+\|\mathrm{d} u\|_{t+s-1}+\|p\|_{t+s-1}\right), \\
& \quad+\left\|p-p_{h}\right\| \leq C h^{t+s-1}\left(\left\|f_{\mathrm{d}}\right\|_{t-1}+\|p\|_{t+s-1}\right) .
\end{aligned}
$$

If $\mathfrak{H}_{h}^{k} \subset \mathfrak{H}^{k}$, then the term $\left\|u_{\mathrm{d}}\right\|_{t-1}$ and the corresponding regularity hypothesis on $u_{\mathrm{d}}$ may be omitted. If $f_{\mathrm{d}} \in H^{t+s-1} \Lambda^{k}(\Omega)$, then $\left\|\mathrm{d}\left(\sigma-\sigma_{h}\right)\right\| \leq$ $C h^{t+s-1}\left\|f_{\mathrm{d}}\right\|_{t+s-1}$.

Proof. We begin by using the triangle inequality to gather the results from Lemmas 7.6, 7.7, and 7.8 into the following estimates:

$$
\begin{aligned}
\left\|\sigma-\sigma_{h}\right\| & \leq C\left(\left\|\left(I-P_{h}\right) f\right\|^{1 / 2}\left\|\left(I-P_{h}\right) u^{1}\right\|^{1 / 2}+\left\|\left(I-\tilde{\Pi}_{h}\right) \sigma^{2}\right\|\right), \\
\mathrm{d}\left(\sigma-\sigma_{h}\right) & =\left(I-P_{h}\right) f \\
\left\|u-u_{h}\right\|_{H \Lambda} & \leq C\left(\left\|\left(I-P_{h}\right) f\right\|^{1 / 2}\left[\left\|\left(I-P_{h}\right) u^{1}\right\|^{1 / 2}+\left\|\left(I-\tilde{\Pi}_{h}\right) w^{h}\right\|^{1 / 2}\right]\right. \\
\left.+\left\|\left(I-\tilde{\Pi}_{h}\right) u^{2}\right\|_{H \Lambda}+\left\|P_{\mathfrak{H}_{h}^{k}} u^{2}\right\|+\left\|\left(I-\tilde{\Pi}_{h}\right) \sigma^{2}\right\|+\left\|\left(I-\tilde{\Pi}_{h}\right) p^{2}\right\|\right) & \\
\left\|p-p_{h}\right\| & \leq C\left(\left\|\left(I-P_{h}\right) f\right\|^{1 / 2}\left\|\left(I-\tilde{\Pi}_{h}\right) r\right\|^{1 / 2}+\left\|\left(I-\tilde{\Pi}_{h}\right) p^{2}\right\|\right)
\end{aligned}
$$

In the above, $\left(\sigma^{1}, u^{1}, p^{1}\right)$ and $\left(\sigma^{2}, u^{2}, p^{2}\right)$ denote the solutions of problem (7.1) with $f$ replaced by $\left(I-P_{h}\right) f$ and $P_{h} f$, respectively, and $w^{h}$ and $r$ 
are defined as in Lemma 7.7. Next recall that for any $g \in H^{s} \Lambda^{k}(\Omega)$, with $0 \leq s \leq r$, we have

$$
\left\|\left(I-\tilde{\Pi}_{h}\right) g\right\| \leq C h^{s}\|g\|_{s} .
$$

We first estimate $\left\|\left(I-P_{h}\right) u^{1}\right\|$. Since $u^{1} \in \mathfrak{B}^{k}, \tilde{\Pi}_{h} u_{1} \in \mathfrak{B}_{h}^{k}$, so

$$
\left\|\left(I-P_{h}\right) u^{1}\right\| \leq\left\|\left(I-\tilde{\Pi}_{h}\right) u^{1}\right\| \leq C h^{s}\left\|u^{1}\right\|_{s} \leq C h^{s}\left\|\delta u^{1}\right\|=C h^{s}\left\|\sigma^{1}\right\|,
$$

where we have used the fact that $\mathrm{d} u^{1}=0$. Using this result, together with Lemma 7.6, we get

$$
\left\|\sigma^{1}\right\|^{2} \leq\left\|\left(I-P_{h}\right) f\right\|\left\|\left(I-P_{h}\right) u^{1}\right\| \leq C h^{s}\left\|\left(I-P_{h}\right) f\right\|\left\|\sigma^{1}\right\|,
$$

and so

$$
\left\|\sigma^{1}\right\| \leq C h^{s}\left\|\left(I-P_{h}\right) f\right\|, \quad\left\|\left(I-P_{h}\right) u^{1}\right\| \leq C h^{2 s}\left\|\left(I-P_{h}\right) f\right\| .
$$

We next estimate $\left\|\left(I-\tilde{\Pi}_{h}\right) w^{h}\right\|$. Since $w^{h} \in \stackrel{\circ}{\mathfrak{B}}^{* k}$ and $\mathrm{d} w^{h}=\mathrm{d} u_{\delta, h}^{1}$,

$$
\left\|w^{h}-\tilde{\Pi}_{h} w^{h}\right\| \leq C h^{s}\left\|w^{h}\right\|_{s} \leq C h^{s}\left\|\mathrm{~d} w^{h}\right\|=C h^{s}\left\|\mathrm{~d} u_{\delta, h}^{1}\right\| .
$$

By Lemma 7.7,

$\left\|\mathrm{d} u_{\delta, h}^{1}\right\|^{2}=\left\|\mathrm{d} u_{h}^{1}\right\|^{2} \leq C\left\|\left(I-P_{h}\right) f\right\|\left\|\left(I-\tilde{\Pi}_{h}\right) w^{h}\right\| \leq C h^{s}\left\|\left(I-P_{h}\right) f\right\|\left\|\mathrm{d} u_{\delta, h}^{1}\right\|$, and so

$$
\left\|\mathrm{d} u_{\delta, h}^{1}\right\| \leq C h^{s}\left\|\left(I-P_{h}\right) f\right\|, \quad\left\|w^{h}-\tilde{\Pi}_{h} w^{h}\right\| \leq C h^{2 s}\left\|\left(I-P_{h}\right) f\right\| .
$$

Using (7.24), we have

$$
\left\|\left(I-\tilde{\Pi}_{h}\right) r\right\| \leq C h^{s}\|r\|_{s} \leq C h^{s}\|r\| \leq C h^{s}\left\|\left(I-P_{h}\right) f\right\| .
$$

If $\mathfrak{H}_{h}^{k} \subset \mathfrak{H}^{k}$, then since $P_{\mathfrak{H}^{k}} u^{2}=0$, we get $P_{\mathfrak{H}_{h}^{k}} u^{2}=0$. Otherwise, an estimate similar to the one above shows that $\varepsilon_{h} \leq C h^{s}$ and so using (7.18), we obtain

$$
\left\|P_{\mathfrak{H}_{h}^{k}} u^{2}\right\| \leq \varepsilon_{h}\left\|\left(I-\tilde{\Pi}_{h}\right) u_{d}^{2}\right\| \leq C h^{s}\left\|\left(I-\tilde{\Pi}_{h}\right) u_{d}^{2}\right\| .
$$

To obtain estimates in terms of our original variables, we next observe that

$$
\begin{aligned}
\left\|\left(I-\tilde{\Pi}_{h}\right) \sigma^{2}\right\| & \leq\left\|\left(I-\tilde{\Pi}_{h}\right) \sigma\right\|+\left\|\left(I-\tilde{\Pi}_{h}\right) \sigma^{1}\right\| \leq\left\|\left(I-\tilde{\Pi}_{h}\right) \sigma\right\|+C\left\|\sigma^{1}\right\| \\
& \leq\left\|\left(I-\tilde{\Pi}_{h}\right) \sigma\right\|+C h^{s}\left\|\left(I-P_{h}\right) f\right\|,
\end{aligned}
$$

and

$$
\begin{aligned}
\left\|\left(I-\tilde{\Pi}_{h}\right) u^{2}\right\| & \leq\left\|\left(I-\tilde{\Pi}_{h}\right) u\right\|+\left\|\left(I-\tilde{\Pi}_{h}\right) u^{1}\right\| \\
& \leq\left\|\left(I-\tilde{\Pi}_{h}\right) u\right\|+C h^{2 s}\left\|\left(I-P_{h}\right) f\right\| .
\end{aligned}
$$

Since $u^{1} \in \mathfrak{B}^{k}$, a similar argument shows that

$$
\left\|\left(I-\tilde{\Pi}_{h}\right) u_{d}^{2}\right\| \leq\left\|\left(I-\tilde{\Pi}_{h}\right) u_{d}\right\|+C h^{2 s}\left\|\left(I-P_{h}\right) f\right\| .
$$


By observing that $\mathrm{d} u^{2}=\mathrm{d} u$ and $p^{2}=p$, and combining the estimates above with (7.26) applied to $u^{2}$, we obtain, after inserting all these results in $(7.25)$,

$$
\begin{gathered}
\left\|\sigma-\sigma_{h}\right\| \leq C\left(h^{s}\left\|\left(I-P_{h}\right) f\right\|+\left\|\left(I-\tilde{\Pi}_{h}\right) \sigma\right\|\right) \\
\left\|u-u_{h}\right\|_{H \Lambda} \leq C\left(h^{s}\left\|\left(I-P_{h}\right) f\right\|+\left\|\left(I-\tilde{\Pi}_{h}\right) u\right\|_{H \Lambda}\right. \\
\left.\quad+\left\|\left(I-\tilde{\Pi}_{h}\right) \sigma\right\|+\left\|\left(I-\tilde{\Pi}_{h}\right) p\right\|+h^{s}\left\|\left(I-\tilde{\Pi}_{h}\right) u_{d}\right\|\right) \\
\left\|p-p_{h}\right\| \leq C\left(h^{s}\left\|\left(I-P_{h}\right) f\right\|+\left\|\left(I-\tilde{\Pi}_{h}\right) p\right\|\right) .
\end{gathered}
$$

As noted above, the term $h^{s}\left\|\left(I-\tilde{\Pi}_{h}\right) u_{d}\right\|$ will not appear if $\mathfrak{H}_{h}^{k} \subset \mathfrak{H}^{k}$. We now estimate the remaining terms. Since $\delta \sigma=0, \mathrm{~d} \sigma=f_{\mathrm{d}}$, and $\operatorname{Tr} \star u=0$ implies

$$
\operatorname{Tr} \star \sigma=\operatorname{Tr} \star \delta u=\operatorname{Tr}\left[(-1)^{k} \mathrm{~d} \star u\right]=(-1)^{k} \mathrm{~d}_{\Gamma} \operatorname{Tr} \star u=0,
$$

we may again use (7.24) to obtain

$$
\left\|\left(I-\tilde{\Pi}_{h}\right) \sigma\right\| \leq C h^{s}\|\sigma\|_{s} \leq C h^{s}\left\|f_{\mathrm{d}}\right\| \leq C h^{s}\|f\| .
$$

To estimate the term $\left\|\left(I-\Pi_{h}\right) u\right\|_{H \Lambda}$, we note that $u$ satisfies $\delta \mathrm{d} u=f_{\delta}$ and $\operatorname{Tr} \star \mathrm{d} u=0$. Applying (7.24), we get

$$
\|\mathrm{d} u\|_{s} \leq C\left\|f_{\delta}\right\| \leq C\|f\| \text {. }
$$

Since $\operatorname{Tr} \star u=0$ and $u \perp \mathfrak{H}^{k}$, we also have by (7.24) and Theorem 7.2

$$
\|u\|_{s} \leq C(\|\mathrm{~d} u\|+\|\delta u\|) \leq C(\|\mathrm{~d} u\|+\|\sigma\|) \leq C\|f\| .
$$

Applying our approximation results, we get

$$
\left\|u-\tilde{\Pi}_{h} u\right\| \leq C h^{s}\|u\|_{s} \leq C h^{s}\|f\|
$$

and

$$
\left\|\mathrm{d} u-\mathrm{d} \tilde{\Pi}_{h} u\right\|=\left\|\left(I-\tilde{\Pi}_{h}\right) \mathrm{d} u\right\| \leq C h^{s}\|\mathrm{~d} u\|_{s} \leq C h^{s}\|f\| .
$$

Again using (7.24), we have

$$
\left\|\left(I-\tilde{\Pi}_{h}\right) p\right\| \leq C h^{s}\|p\|_{s} \leq C h^{s}\|p\| \leq C h^{s}\left\|f_{\mathfrak{H}}\right\| \leq C h^{s}\|f\| .
$$

Finally, we note that

$$
\left\|\left(I-P_{h}\right) f\right\| \leq\|f\|, \quad\left\|\left(I-\tilde{\Pi}_{h}\right) u_{d}\right\| \leq C\left\|u_{d}\right\| \leq C\|f\| .
$$

Using these results in (7.27) establishes the first statement of the theorem. The second statement also follows from (7.27), using the fact that $\|(I-$ $\left.P_{h}\right) f\|\leq\|\left(I-\tilde{\Pi}_{h}\right) f_{\mathrm{d}} \|$ and the approximation properties of the projection operators $\tilde{\Pi}_{h}$. The final estimate in the theorem follows directly from the second estimate in (7.25). 


\section{Eigenvalue problems}

The purpose of this section is to discuss approximations of the eigenvalue problem for the Hodge Laplacian using the same finite element spaces $\Lambda_{h}^{k} \subset H \Lambda^{k}(\Omega)$ used for the boundary value problems discussed in the previous section. In fact, there is a vast literature on the approximation of eigenvalue problems for mixed systems, and it is well known that the standard Brezzi stability conditions for linear saddle point systems are not sufficient to guarantee convergence of the corresponding eigenvalue approximations. Typically, spurious eigenvalues/eigenfunctions can occur, even if the Brezzi conditions are fulfilled. However, by now the proper conditions for guaranteeing convergence and no spurious eigenmodes are well understood, and discrete Hodge (or Helmholtz) decompositions seem to be a useful tool for verifying these conditions. For the main results in this direction, we refer to Osborn (1979), Mercier, Osborn, Rappaz and Raviart (1981), Babuška and Osborn (1991), Boffi, Brezzi and Gastaldi (1997), Boffi, Brezzi and Gastaldi (2000), Boffi (2000), Boffi (2001), and Boffi (2006).

Here, we shall use this theory to show that the finite element spaces defined in this paper lead to convergence of the corresponding finite element approximations of the eigenvalue problem for the Hodge Laplacian. In the final subsection, we present some results on convergence rates for the special case of a simple eigenvalue.

\subsection{The eigenvalue problem for the Hodge Laplacian}

We shall only consider the eigenvalue problem with boundary conditions which are natural with respect to the mixed formulation, i.e., the boundary conditions are $\operatorname{Tr}(\star u)=0$ and $\operatorname{Tr}(\star \mathrm{d} u)=0$. The eigenvalue problem for the Hodge Laplacian then takes the following form.

Find $\lambda \in \mathbb{R}$ and $(\sigma, u) \in H \Lambda^{k-1}(\Omega) \times H \Lambda^{k}(\Omega)$ such that

$$
\begin{aligned}
\langle\sigma, \tau\rangle-\langle\mathrm{d} \tau, u\rangle & =0, & & \tau \in H \Lambda^{k-1}(\Omega), \\
\langle\mathrm{d} \sigma, v\rangle+\langle\mathrm{d} u, \mathrm{~d} v\rangle & =\lambda\langle u, v\rangle, & & v \in H \Lambda^{k}(\Omega),
\end{aligned}
$$

where $(\sigma, u)$ is not identically zero. We remark that standard symmetry arguments show that only real eigenvalues are possible for this problem: see (8.5) below. Also, the identity

$$
\|\sigma\|^{2}+\|\mathrm{d} u\|^{2}=\lambda\|u\|^{2}
$$

implies that all eigenvalues are nonnegative.

The first equation above expresses that $\delta u=\sigma \in L^{2} \Lambda^{k}(\Omega)$ and that $\operatorname{Tr}(\star u)=0$. Hence, $u \in H \Lambda^{k}(\Omega) \cap \stackrel{\circ}{*}^{*} \Lambda^{k}(\Omega)$. The eigenvalue problem (8.1) can therefore alternatively be written as follows. 
Find $u \in H \Lambda^{k}(\Omega) \cap \stackrel{\circ}{H}^{*} \Lambda^{k}(\Omega)$ such that

$$
\langle\delta u, \delta v\rangle+\langle\mathrm{d} u, \mathrm{~d} v\rangle=\lambda\langle u, v\rangle, \quad v \in H \Lambda^{k}(\Omega) \cap \stackrel{\circ}{H}^{*} \Lambda^{k}(\Omega) .
$$

In general, $\lambda=0$ will be an eigenvalue for this problem with the space of harmonic $k$-forms, $\mathfrak{H}^{k}$, as the corresponding eigenspace. In fact, just as we split the source problem into independent problems, we may also split the eigenvalue problem (8.1) into three independent eigenvalue problems. To see this, assume that $(\lambda, \sigma, u)$ satisfies $(8.1)$ with $(\sigma, u)$ not identically equal to zero. Decompose

$$
(\sigma, u)=\left(\sigma, u_{\mathrm{d}}\right)+\left(0, u_{\mathfrak{H}}\right)+\left(0, u_{\delta}\right)
$$

where $u_{\mathrm{d}}$ and $u_{\mathfrak{H}}$ are the projections of $u$ onto $\mathfrak{B}^{k}$ and $\mathfrak{H}^{k}$, respectively, with respect to the inner product of $L^{2} \Lambda^{k}(\Omega)$. If $\lambda=0$ then $\sigma=0$ and $u=u_{\mathfrak{H}}$. On the other hand, if $\lambda>0$ then $\lambda\left\|u_{\mathfrak{H}}\right\|^{2}=0$, and therefore $u_{\mathfrak{H}}=0$. Furthermore, $u_{\delta} \in \mathfrak{Z}^{k \perp}$ satisfies

$$
\left\langle\mathrm{d} u_{\delta}, \mathrm{d} v\right\rangle=\lambda\left\langle u_{\delta}, v\right\rangle, \quad v \in \mathfrak{Z}^{k \perp} .
$$

Finally, $\left(\sigma, u_{\mathrm{d}}\right)$ satisfies the system

$$
\begin{aligned}
\langle\sigma, \tau\rangle-\left\langle\mathrm{d} \tau, u_{\mathrm{d}}\right\rangle & =0, & & \tau \in H \Lambda^{k-1}(\Omega), \\
\langle\mathrm{d} \sigma, v\rangle & =\lambda\left\langle u_{\mathrm{d}}, v\right\rangle, & & v \in \mathfrak{B}^{k} .
\end{aligned}
$$

Observe that $\left(\sigma, u_{\mathrm{d}}\right), u_{\mathfrak{H}}$, or $u_{\delta}$ may very well be identically equal to zero, even if $(\sigma, u)$ is nonzero. On the other hand, any eigenvalue/eigenvector of (8.2) or (8.3) corresponds to an eigenvalue/eigenvector of (8.1).

We will introduce $K$ as the solution operator for the Hodge Laplacian. More precisely, for a given $f \in L^{2} \Lambda^{k}(\Omega)$, consider the following problem.

Find $(\sigma, u, p) \in H \Lambda^{k-1}(\Omega) \times H \Lambda^{k}(\Omega) \times \mathfrak{H}^{k}$ such that

$$
\begin{aligned}
\langle\sigma, \tau\rangle-\langle\mathrm{d} \tau, u\rangle & =0, & & \tau \in H \Lambda^{k-1}(\Omega), \\
\langle\mathrm{d} \sigma, v\rangle+\langle\mathrm{d} u, \mathrm{~d} v\rangle+\langle v, p\rangle & =\langle f, v\rangle, & & v \in H \Lambda^{k}(\Omega), \\
\langle u, q\rangle & =0, & & q \in \mathfrak{H}^{k} .
\end{aligned}
$$

The solution operator $K: L^{2} \Lambda^{k}(\Omega) \rightarrow H \Lambda^{k}(\Omega) \cap \stackrel{\circ}{H}^{*} \Lambda^{k}(\Omega)$ is given by

$$
K: f \mapsto K f=u+p .
$$

Note that in (8.4), $p$ is the $L^{2}$-projection of $u+p$ onto $\mathfrak{H}^{k}$. Therefore, an alternative characterization of the operator $K$ is $K f=u^{\prime}$, where $u^{\prime} \in$ $H \Lambda^{k}(\Omega) \cap \stackrel{\circ}{H}^{*} \Lambda^{k}(\Omega)$ solves the system

$$
\left\langle\delta u^{\prime}, \delta v\right\rangle+\left\langle\mathrm{d} u^{\prime}, \mathrm{d} v\right\rangle+\left\langle u_{\mathfrak{H}}^{\prime}, v_{\mathfrak{H}}\right\rangle=\langle f, v\rangle, \quad v \in H \Lambda^{k}(\Omega) \cap \stackrel{\circ}{H}^{*} \Lambda^{k}(\Omega),
$$

where, as above, $v_{\mathfrak{H}}$ is the $L^{2}$-projection of $v$ onto $\mathfrak{H}^{k}$. The operator $K$ is the identity on $\mathfrak{H}^{k}$, and if $f_{\mathfrak{H}}=0$ then $(K f)_{\mathfrak{H}}=0$. Observe also that the 
operator $K$ is symmetric and positive definite on $L^{2} \Lambda^{k}(\Omega)$ since

$$
\langle f, K g\rangle=\langle\delta K f, \delta K g\rangle+\langle\mathrm{d} K f, \mathrm{~d} K g\rangle+\left\langle(K f)_{\mathfrak{H},},(K g)_{\mathfrak{H}}\right\rangle
$$

for all $f, g \in L^{2} \Lambda^{k}(\Omega)$. Furthermore, as a consequence of Theorem 2.1, the operator $K$ is a compact operator in $\mathcal{L}\left(L^{2} \Lambda^{k}(\Omega), L^{2} \Lambda^{k}(\Omega)\right)$.

If $\lambda>0$ and $u \in H \Lambda^{k}(\Omega) \cap \stackrel{\circ}{H}^{*} \Lambda^{k}(\Omega)$ corresponds to an eigenvalue/eigenvector for (8.1) then

$$
K u=\lambda^{-1} u,
$$

and $u_{\mathfrak{H}}=0$. On the other hand, if $u \in \mathfrak{H}^{k}$ then $u$ and $\lambda=1$ satisfy (8.6). In fact, the two eigenvalue problems (8.1) and (8.6) are equivalent, if we just recall that the eigenvalue $\lambda=0$ in (8.1), corresponding to the eigenspace $\mathfrak{H}^{k}$, is shifted to $\lambda=1$ in (8.6). Since $K$ is compact in $\mathcal{L}\left(L^{2} \Lambda^{k}(\Omega), L^{2} \Lambda^{k}(\Omega)\right)$, and not of finite rank, we conclude that the Hodge-Laplace problem (8.1) has a countable set of nonnegative eigenvalues

$$
0 \leq \lambda_{1} \leq \lambda_{2} \leq \cdots \leq \lambda_{j} \leq \cdots
$$

such that $\lim _{j \rightarrow \infty} \lambda_{j}=\infty$.

\subsection{The discrete eigenvalue problem}

In order to approximate the eigenvalue problem for the Hodge Laplacian (8.1), we need to introduce finite element spaces $\Lambda_{h}^{k-1}$ and $\Lambda_{h}^{k}$, which are subspaces of the corresponding Sobolev spaces $H \Lambda^{k-1}(\Omega)$ and $H \Lambda^{k}(\Omega)$ occurring in the formulation of (8.1). We will continue to assume that these discrete spaces satisfy (7.10), (7.11), and (7.12). Given the discrete spaces, the corresponding discrete eigenvalue problem takes the following form.

Find $\lambda_{h} \in \mathbb{R}$ and $\left(\sigma_{h}, u_{h}\right) \in \Lambda_{h}^{k-1} \times \Lambda_{h}^{k},\left(\sigma_{h}, u_{h}\right)$ not identically zero, such that

$$
\begin{aligned}
\left\langle\sigma_{h}, \tau\right\rangle-\left\langle\mathrm{d} \tau, u_{h}\right\rangle & =0, & & \tau \in \Lambda_{h}^{k-1}, \\
\left\langle\mathrm{~d} \sigma_{h}, v\right\rangle+\left\langle\mathrm{d} u_{h}, \mathrm{~d} v\right\rangle & =\lambda_{h}\left\langle u_{h}, v\right\rangle, & & v \in \Lambda_{h}^{k} .
\end{aligned}
$$

If we define a discrete coderivative operator $\mathrm{d}_{h}^{*}: \Lambda_{h}^{k} \rightarrow \Lambda_{h}^{k-1}$ by

$$
\left\langle\mathrm{d}_{h}^{*} \omega, \tau\right\rangle=\langle\mathrm{d} \tau, \omega\rangle, \quad \tau \in \Lambda_{h}^{k-1},
$$

then the first equation states that $\sigma_{h}=\mathrm{d}_{h}^{*} u_{h}$. Note that this identity also contains the information that $\operatorname{Tr}\left(\star u_{h}\right)$ is 'weakly zero', i.e., the boundary condition $\operatorname{Tr}(\star u)$ is approximated as a natural boundary condition.

As in the continuous case above, it follows by a straightforward energy argument that if $\left(\lambda_{h}, \sigma_{h}, u_{h}\right)$ solves (8.7), then

$$
\left\|\sigma_{h}\right\|^{2}+\left\|\mathrm{d} u_{h}\right\|^{2}=\lambda_{h}\left\|u_{h}\right\|^{2}
$$

Therefore, all discrete eigenvalues $\lambda_{h}$ are nonnegative. Furthermore, the 
eigenspace for the eigenvalue $\lambda_{h}=0$ corresponds exactly to the space $\mathfrak{H}_{h}^{k}$ of discrete harmonic $k$-forms. We can assume that the discrete eigenvalues are ordered such that

$$
0 \leq \lambda_{1, h} \leq \lambda_{2, h} \leq \cdots \leq \lambda_{N(h), h},
$$

where $N(h)$ is the dimension of the space $\Lambda_{h}^{k}$.

An alternative characterization of the eigenvalue problem is obtained by introducing a discrete solution operator $K_{h}: L^{2} \Lambda^{k}(\Omega) \rightarrow \Lambda_{h}^{k}$. In parallel to the discussion in the continuous case, we define $K_{h} f=u_{h}+p_{h}$ if $\left(\sigma_{h}, u_{h}, p_{h}\right) \in \Lambda_{h}^{k-1} \times \Lambda_{h}^{k} \times \mathfrak{H}_{h}^{k}$ is the solution of the problem

$$
\begin{aligned}
\left\langle\sigma_{h}, \tau\right\rangle-\left\langle\mathrm{d} \tau, u_{h}\right\rangle & =0, & & \tau \in \Lambda_{h}^{k-1}, \\
\left\langle\mathrm{~d} \sigma_{h}, v\right\rangle+\left\langle\mathrm{d} u_{h}, \mathrm{~d} v\right\rangle+\left\langle v, p_{h}\right\rangle & =\langle f, v\rangle, & & v \in \Lambda_{h}^{k}, \\
\left\langle u_{h}, q\right\rangle & =0, & & q \in \mathfrak{H}_{h}^{k} .
\end{aligned}
$$

Here $\sigma_{h}=\mathrm{d}_{h}^{*} u_{h}=\mathrm{d}_{h}^{*} K_{h} f$. The operator $K_{h}$ is equivalently characterized by $K_{h} f=u_{h}^{\prime}$, where $u_{h}^{\prime} \in \Lambda_{h}^{k}$ solves

$$
\left\langle\delta u_{h}^{\prime}, \delta v\right\rangle+\left\langle\mathrm{d} u_{h}^{\prime}, \mathrm{d} v\right\rangle+\left\langle u_{\mathfrak{H}, h}^{\prime}, v_{\mathfrak{H}, h}\right\rangle=\langle f, v\rangle, \quad v \in \Lambda_{h}^{k},
$$

where $v_{\mathfrak{H}, h}$ denotes the $L^{2}$-projection of $v$ onto $\mathfrak{H}_{h}^{k}$.

The eigenvalue problem (8.7) is equivalent to the corresponding eigenvalue problem for the operator $K_{h}$ given by

$$
K_{h} u_{h}=\lambda_{h}^{-1} u_{h}
$$

with the interpretation that the eigenvalue $\lambda_{h}=0$ in (8.7) is shifted to $\lambda_{h}=1$ in (8.8). The discrete operator $K_{h}$ is again symmetric on $L^{2} \Lambda^{k}(\Omega)$ since it is straightforward to verify that

$$
\left\langle f, K_{h} g\right\rangle=\left\langle\mathrm{d}_{h}^{*} K_{h} f, \mathrm{~d}_{h}^{*} K_{h} g\right\rangle+\left\langle\mathrm{d} K_{h} f, \mathrm{~d} K_{h} g\right\rangle+\left\langle\left(K_{h} f\right)_{\mathfrak{H}, h},\left(K_{h} g\right)_{\mathfrak{H}, h}\right\rangle
$$

for all $f, g \in L^{2} \Lambda^{k}(\Omega)$.

\subsection{Convergence of the discrete approximations}

For every positive integer $j$, we let $m(j)$ be the dimension of the eigenspace spanned by the first $j$ distinct eigenvalues of the Hodge-Laplace problem (8.1). We let $\mathfrak{E}_{i}$ denote the eigenspace associated to the eigenvalue $\lambda_{i}$, while $\mathfrak{E}_{i, h}$ is the corresponding discrete eigenspace associated to $\lambda_{i, h}$.

The discrete eigenvalue problem (8.7) is said to converge to the exact eigenvalue problem (8.1) if, for any $\epsilon>0$ and integer $j>0$, there exists a 
mesh parameter $h_{0}>0$ such that, for all $h \leq h_{0}$, we have

$$
\begin{array}{r}
\max _{1 \leq i \leq m(j)}\left|\lambda_{i}-\lambda_{i, h}\right| \leq \epsilon, \\
\operatorname{gap}\left(\bigoplus_{i=1}^{m(j)} \mathfrak{E}_{i}, \bigoplus_{i=1}^{m(j)} \mathfrak{E}_{i, h}\right) \leq \epsilon .
\end{array}
$$

Here gap $=\operatorname{gap}(E, F)$ is the gap between two subspaces $E$ and $F$ of a Hilbert space $H$ given by

$$
\operatorname{gap}(E, F)=\max \left(\sup _{\substack{u \in E \\\|u\|_{H}=1}} \inf _{v \in F}\|u-v\|_{H}, \sup _{\substack{v \in F \\\|v\|_{H}=1}} \inf _{u \in E}\|u-v\|_{H}\right) .
$$

This is a reasonable concept of convergence since, besides convergence of the eigenmodes, it also contains the information that no spurious eigenmodes pollute the spectrum. Furthermore, for eigenvalue problems of the form (8.6) and (8.8), which are equivalent to (8.1) and (8.7), convergence will follow if the operators $K_{h}$ converge to $K$ in the operator norm. In other words, if

$$
\left\|K_{h}-K\right\|_{\mathcal{L}\left(L^{2} \Lambda^{k}(\Omega), L^{2} \Lambda^{k}(\Omega)\right)} \longrightarrow 0 \quad \text { as } h \rightarrow 0,
$$

then the discrete eigenvalue problem (8.7) converges to the eigenvalue problem (8.1) in the sense specified above: see Kato (1995, Chapter IV). Here, the gap between the subspaces is defined with respect to the Hilbert space $L^{2} \Lambda^{k}(\Omega)$. In fact, it was established in Boffi et al. (2000) that this operator convergence is both sufficient and necessary for obtaining convergence of the eigenvalue approximations.

As a consequence, in the present case it only remains to estimate $\| K_{h}-$ $K \|_{\mathcal{L}\left(L^{2} \Lambda^{k}(\Omega), L^{2} \Lambda^{k}(\Omega)\right)}$. However, it is a direct consequence of Theorem 7.9 and the definitions of the operator $K$ and $K_{h}$ above that

$$
\left\|K_{h}-K\right\|_{\mathcal{L}\left(L^{2} \Lambda^{k}(\Omega), L^{2} \Lambda^{k}(\Omega)\right)} \leq c h^{s},
$$

where $s \geq 1 / 2$ and the constant $c$ is independent of $h$. Therefore, the convergence property (8.9) holds and convergence of the eigenvalues and eigenvectors are guaranteed.

\section{Convergence}

As a consequence of (8.10) and the discussion above the following theorem is obtained.

Theorem 8.1. The discrete eigenvalue problems (8.7) converges to the eigenvalue problem (8.1) in the sense defined above.

In particular, this theorem implies the following result on the approximation of the $k$-harmonic forms. 


\section{Corollary 8.2.}

$$
\lim _{h \rightarrow 0} \operatorname{gap}\left(\mathfrak{H}^{k}, \mathfrak{H}_{h}^{k}\right)=0 .
$$

Recall that for the continuous problem (8.1), all eigenfunctions corresponding to eigenvalues $\lambda>0$ are $L^{2}$-orthogonal to $\mathfrak{H}^{k}$, and for such eigenfunctions, the problem (8.1) can be split into two independent problems (8.2) and (8.3). The corresponding property is valid for the discrete problem (8.7) as well. If $\lambda_{h}>0$, then all eigenfunctions are $L^{2}$-orthogonal to $\mathfrak{H}_{h}^{k}$, and if $u_{h}=u_{\mathrm{d}, h}+u_{\delta, h}$ is the discrete Hodge decomposition of an eigenfunction $u_{h}$ in $\Lambda_{h}^{k}$ then

$$
\left\langle\mathrm{d} u_{\delta, h}, \mathrm{~d} v\right\rangle=\lambda_{h}\left\langle u_{\delta, h}, v\right\rangle, \quad v \in \mathfrak{Z}_{h}^{k \perp},
$$

and

$$
\begin{aligned}
\left\langle\sigma_{h}, \tau\right\rangle-\left\langle\mathrm{d} \tau, u_{\mathrm{d}, h}\right\rangle & =0, & & \tau \in \Lambda_{h}^{k-1}, \\
\left\langle\mathrm{~d} \sigma_{h}, v\right\rangle & =\lambda_{h}\left\langle u_{\mathrm{d}, h}, v\right\rangle, & & v \in \mathfrak{B}_{h}^{k} .
\end{aligned}
$$

Here $\sigma_{h}=\mathrm{d}_{h}^{*} u_{h}$. The converse also holds, i.e., any eigenvalue/eigenfunction of (8.11) or (8.12) is an eigenvalue/eigenfunction of (8.7). In fact the discrete problems (8.11) and (8.12) converges separately to the eigenvalue problems (8.2) and (8.3) in the sense specified above. This is basically a consequence of Theorem 8.1 and the orthogonality property of the Hodge decompositions. To see this the following result is useful.

Lemma 8.3. If $u \in \mathfrak{B}^{k}$ and $v \in \operatorname{span}\left(\mathfrak{Z}^{k \perp}, \mathfrak{Z}_{h}^{k \perp}\right)$ then the bounds

$$
\|u-v\| \geq\|v\|-\left\|\left(I-\tilde{\Pi}_{h}\right) u\right\|, \quad \text { and }\|u-v\| \geq\|u\|-2\left\|\left(I-\tilde{\Pi}_{h}\right) u\right\|
$$

holds.

Proof. Since $\tilde{\Pi}_{h} u \in \mathfrak{B}_{h}^{k} \subset \mathfrak{B}^{k}$ and $v$ are orthogonal we have

$$
\begin{aligned}
\|u-v\| & \geq\left\|\tilde{\Pi}_{h} u-v\right\|-\left\|\left(I-\tilde{\Pi}_{h}\right) u\right\| \\
& \geq \max \left(\left\|\tilde{\Pi}_{h} u\right\|,\|v\|\right)-\left\|\left(I-\tilde{\Pi}_{h}\right) u\right\| .
\end{aligned}
$$

This gives the first inequality, and the second bound follows since $\left\|\tilde{\Pi}_{h} u\right\| \geq$ $\|u\|-\left\|\left(I-\tilde{\Pi}_{h}\right) u\right\|$.

We now have the following result which is a strengthening of Theorem 8.1.

Theorem 8.4. The discrete eigenvalue problems (8.11) and (8.12) converges separately to the corresponding problems (8.2) and (8.3) in the sense defined above.

Proof. Let $\lambda>0$ be an eigenvalue for the problem (8.1) with corresponding eigenspace $\mathfrak{E}$, and $\lambda_{h}$ the corresponding eigenvalue for (8.7), with eigenspace $\mathfrak{E}_{h}$, such that $\lambda_{h} \rightarrow \lambda$ and $\operatorname{gap}\left(\mathfrak{E}, \mathfrak{E}_{h}\right) \rightarrow 0$ as $h$ tends to zero. Let

$$
\mathfrak{E}=\mathfrak{E}_{\mathrm{d}} \oplus \mathfrak{E}_{\delta} \quad \text { and } \quad \mathfrak{E}_{h}=\mathfrak{E}_{\mathrm{d}, h} \oplus \mathfrak{E}_{\delta, h}
$$


be the corresponding Hodge decomposition (continuous and discrete) of the spaces, i.e $\mathfrak{E}_{\mathrm{d}}=\mathfrak{E} \cap \mathfrak{B}^{k}$ and $\mathfrak{E}_{\mathrm{d}, h}=\mathfrak{E}_{h} \cap \mathfrak{B}_{h}^{k}$. The desired result will follow if we can show that $\operatorname{gap}\left(\mathfrak{E}_{\mathrm{d}}, \mathfrak{E}_{\mathrm{d}, h}\right) \rightarrow 0$ and $\operatorname{gap}\left(\mathfrak{E}_{\delta}, \mathfrak{E}_{\delta, h}\right) \rightarrow 0$.

However, if $u \in \mathfrak{E}_{\mathrm{d}}$, with $\|u\|=1$, then by Lemma 8.3,

$$
\begin{aligned}
\inf _{v \in \mathfrak{E}_{\mathrm{d}, h}}\|u-v\| & \leq \inf _{v \in \mathfrak{E}_{h}}\|u-v\|+2\left\|\left(I-\tilde{\Pi}_{h}\right) u\right\| \\
& \leq \operatorname{gap}\left(\mathfrak{E}, \mathfrak{E}_{h}\right)+2\left\|\left(I-\tilde{\Pi}_{h}\right) u\right\|,
\end{aligned}
$$

and since the space $\mathfrak{E}_{\mathrm{d}}$ is finite-dimensional the right hand side converges to zero uniformly in $u$. On the other hand, for a given $v \in \mathfrak{E}_{\mathrm{d}, h}$, with $\|v\|=1$, we have

$$
\inf _{u \in \mathfrak{E}_{\mathrm{d}}}\|u-v\|=\inf _{u \in \mathfrak{E}}\|u-v\| \leq \operatorname{gap}\left(\mathfrak{E}, \mathfrak{E}_{h}\right) .
$$

This shows that $\operatorname{gap}\left(\mathfrak{E}_{\mathrm{d}}, \mathfrak{E}_{\mathrm{d}, h}\right) \rightarrow 0$ and a corresponding argument will show that $\operatorname{gap}\left(\mathfrak{E}_{\delta}, \mathfrak{E}_{\delta, h}\right) \rightarrow 0$.

\section{Convergence rates}

The results of Section 7 can also be combined with the standard theory for eigenvalue approximation to obtain rates of convergence. To simplify the presentation, we do this only for the simplest case, where we assume we have only simple eigenvalues. Then, from Theorem 7.3 of Babuška and Osborn (1991), if $K u=\lambda^{-1} u,\|u\|=1$, then

$$
\left|\lambda^{-1}-\lambda_{h}^{-1}\right| \leq C\left(\left|\left\langle\left(K-K_{h}\right) u, u\right\rangle\right|+\left\|\left(K-K_{h}\right) u\right\|^{2}\right) .
$$

For the second term, we can use the error estimates of Section 7. To get a similar squaring of the error from the first term, we need some additional analysis, which is a slight modification of Theorem 11.1 of Babuška and Osborn (1991). To use the results of Section 7, and avoid confusing the terminology, we estimate $\left|\left\langle\left(K-K_{h}\right) f, f\right\rangle\right|$, where now $\|f\|=1$ and $(K-$ $\left.K_{h}\right) f=u-u_{h}+p-p_{h}$, i.e., we estimate $\left|\left\langle u-u_{h}+p-p_{h}, f\right\rangle\right|$.

We first note that from the definitions of $\sigma, u$, and $p$, and their finite element approximations, we get the error equations

$$
\begin{aligned}
\left\langle\sigma-\sigma_{h}, \tau\right\rangle=\left\langle\mathrm{d} \tau, u-u_{h}\right\rangle, & \tau \in \Lambda_{h}^{k-1}, \\
\left\langle\mathrm{~d}\left(\sigma-\sigma_{h}\right), v\right\rangle+\left\langle\mathrm{d}\left(u-u_{h}\right), \mathrm{d} v\right\rangle+\left\langle p-p_{h}, v\right\rangle=0, & v \in \Lambda_{h}^{k} .
\end{aligned}
$$

Also from the definitions of $\sigma, u$, and $p$, we have

$$
\begin{aligned}
\left\langle f, u-u_{h}+p-p_{h}\right\rangle=\left\langle\mathrm{d} \sigma, u-u_{h}+p-p_{h}\right\rangle+\left\langle\mathrm{d} u, \mathrm{~d}\left(u-u_{h}\right)\right\rangle & \\
& +\left\langle p, u-u_{h}+p-p_{h}\right\rangle, \\
\left\langle\sigma, \sigma-\sigma_{h}\right\rangle= & \left\langle\mathrm{d}\left(\sigma-\sigma_{h}\right), u\right\rangle .
\end{aligned}
$$


Since $\langle\mathrm{d} \sigma, p\rangle=\left\langle\mathrm{d} \sigma_{h}, p\right\rangle=0$, we obtain

$$
\begin{aligned}
& \left\langle f, u-u_{h}+p-p_{h}\right\rangle=\left\langle\mathrm{d} \sigma, u-u_{h}+p-p_{h}\right\rangle-\left\langle\sigma, \sigma-\sigma_{h}\right\rangle \\
& +\left\langle\mathrm{d}\left(\sigma-\sigma_{h}\right), u+p\right\rangle+\left\langle\mathrm{d} u, \mathrm{~d}\left(u-u_{h}\right)\right\rangle+\left\langle p-p_{h}, p+u\right\rangle \\
& +\left\langle p, u-u_{h}\right\rangle-\left\langle p-p_{h}, u\right\rangle .
\end{aligned}
$$

Since $\langle u, q\rangle=0, q \in \mathfrak{H}^{k}$ and $\left\langle u_{h}, q_{h}\right\rangle=0, q_{h} \in \mathfrak{H}_{h}^{k}$,

$$
\left\langle p, u-u_{h}\right\rangle-\left\langle p-p_{h}, u\right\rangle=\left\langle p-p_{h}, u-u_{h}\right\rangle+2\left\langle p_{h}, u\right\rangle .
$$

Using the Hodge decomposition $u=u_{\mathrm{d}}+u_{\mathfrak{H}}+u_{\delta}$, and observing that $u_{\mathfrak{H}}=0$, $\left\langle p_{h}, u_{\delta}\right\rangle=0$, and $\tilde{\Pi}_{h} u_{\mathrm{d}} \in \mathfrak{B}_{h}^{k} \subset \mathfrak{B}^{k}$, we get

$$
\left\langle p_{h}, u\right\rangle=\left\langle p_{h}, u_{\mathrm{d}}\right\rangle=\left\langle p_{h}, u_{\mathrm{d}}-\Pi_{h} u_{\mathrm{d}}\right\rangle=\left\langle p_{h}-p, u_{\mathrm{d}}-\Pi_{h} u_{\mathrm{d}}\right\rangle .
$$

Choosing $\tau=\sigma_{h}$ and $v=u_{h}+p_{h}$ in (8.13) and (8.14), and combining these results, we get

$$
\begin{aligned}
& \left\langle f, u-u_{h}+p-p_{h}\right\rangle=\left\langle\mathrm{d}\left(\sigma-\sigma_{h}\right), u-u_{h}+p-p_{h}\right\rangle-\left\langle\sigma-\sigma_{h}, \sigma-\sigma_{h}\right\rangle \\
& +\left\langle\mathrm{d}\left(\sigma-\sigma_{h}\right), u-u_{h}+p-p_{h}\right\rangle+\left\langle\mathrm{d}\left(u-u_{h}\right), \mathrm{d}\left(u-u_{h}\right)\right\rangle \\
& +\left\langle p-p_{h}, u-u_{h}+p-p_{h}\right\rangle+\left\langle p-p_{h}, u-u_{h}\right\rangle+2\left\langle p_{h}-p, u_{\mathrm{d}}-\tilde{\Pi}_{h} u_{\mathrm{d}}\right\rangle .
\end{aligned}
$$

Hence,

$$
\begin{aligned}
\left|\left\langle f, u-u_{h}+p-p_{h}\right\rangle\right| \leq C(\| \sigma- & \sigma_{h}\left\|_{H \Lambda}+\right\| u-u_{h} \|_{H \Lambda} \\
& \left.+\left\|p-p_{h}\right\|\right)^{2}+2\left\|p_{h}-p\right\|\left\|u_{\mathrm{d}}-\tilde{\Pi}_{h} u_{\mathrm{d}}\right\| .
\end{aligned}
$$

Applying our approximation theory results and Theorem 7.9, we get in the case when $s=1$ and the eigenfunction is sufficiently smooth, that

$$
\left|\left\langle f, u-u_{h}+p-p_{h}\right\rangle\right| \leq C h^{2 r}
$$

and hence it follows that

$$
\left|\lambda-\lambda_{h}\right| \leq C h^{2 r}
$$

\section{The $H \Lambda$ projection and Maxwell's equations}

In this section, we consider the approximation of variations of the following problem.

Given $f \in L^{2} \Lambda^{k}(\Omega)$, find $u \in H \Lambda^{k}(\Omega)$ satisfying

$$
\langle u, v\rangle_{H \Lambda}:=\langle\mathrm{d} u, \mathrm{~d} v\rangle+\langle u, v\rangle=\langle f, v\rangle, \quad v \in H \Lambda^{k}(\Omega) .
$$

Note that when $k=0$, this corresponds to a perturbation of the HodgeLaplace problem we have considered previously, by adding a lower order term. Problems of this form will also arise later in this paper in the section on preconditioning. The time-harmonic Maxwell's equations can be written 
as a variation of this form, where $k=1$ and the term $\langle u, v\rangle$ is replaced by $-m^{2}\langle u, v\rangle$. We will study this case below, when $m^{2}$ is not an eigenvalue, i.e., when there is no nonzero $u \in H \Lambda^{k}(\Omega)$ for which $\langle\mathrm{d} u, \mathrm{~d} v\rangle=m^{2}\langle u, v\rangle$ for all $v \in H \Lambda^{k}(\Omega)$.

A simple approximation scheme for (9.1) is to seek $u_{h} \in \Lambda_{h}^{k}$ satisfying

$$
\left\langle u_{h}, v\right\rangle_{H \Lambda}=\langle f, v\rangle, \quad v \in \Lambda_{h}^{k} .
$$

The error analysis in the $H \Lambda$-norm of such a problem is straightforward. By subtracting (9.2) from (9.1), we get the error equation

$$
\left\langle u-u_{h}, v\right\rangle_{H \Lambda}=0, \quad v \in \Lambda_{h}^{k} .
$$

Hence, $u_{h}$ is the $H \Lambda$-projection of $u$ into $\Lambda_{h}^{k}$ and therefore satisfies the optimal error estimate

$$
\left\|u-u_{h}\right\|_{H \Lambda} \leq \inf _{v \in \Lambda_{h}^{k}}\|u-v\|_{H \Lambda} .
$$

If we modify the problem by replacing the term $\langle u, v\rangle$ is replaced by $m^{2}\langle u, v\rangle$, then the analysis is essentially the same, since $\langle\mathrm{d} u, \mathrm{~d} v\rangle+m^{2}\langle u, v\rangle$ defines an equivalent inner product on $H \Lambda^{k}(\Omega)$.

\subsection{Maxwell-type problems}

We shall also consider the modification of the problem (9.1) obtained by replacing $\langle u, v\rangle$ by $-m^{2}\langle u, v\rangle$. That is, we consider the following problem.

Find $u \in H \Lambda^{k}(\Omega)$ such that

$$
\langle\mathrm{d} u, \mathrm{~d} v\rangle-m^{2}\langle u, v\rangle=\langle f, v\rangle, \quad v \in H \Lambda^{k}(\Omega) .
$$

This problem is indefinite, and its analysis more complicated. We will assume that $m^{2}$ is not an eigenvalue, since otherwise the problem is not wellposed.

Problems of the form (9.4) arise for example, in the study of Maxwell's equations. In the cavity problem for the time-harmonic Maxwell's equations subject to a perfect conducting boundary condition, one seeks the electric field $E$ satisfying

$$
\operatorname{curl}(\operatorname{curl} E)-m^{2} E=F \text { in } \Omega, \quad \nu \times E=0 \text { on } \Gamma=\partial \Omega .
$$

Here $F$ is a given function related to the imposed current sources and the parameter $m$ is the wave number, assumed to be real and positive, and the permittivity and permeability coefficients are set equal to one. This problem corresponds to the problem (9.4) with $k=1$ and $f \in \mathfrak{B}^{* k}$, but with boundary conditions given as $\operatorname{Tr}(u)=0$ and $\operatorname{Tr}(\delta u)=0$. For an extensive treatment of Maxwell's equation using finite element exterior calculus, see the paper of Hiptmair (2002). A comprehensive treatment in standard finite element notation can be found in the book of Monk (2003a). 
As in the case of the time-harmonic Maxwell's equation, problems of the form (9.4) arise by considering the steady state problem obtained from the ansatz of a time-harmonic solution to the following second order timedependent differential equation related to the Hodge Laplacian:

$$
\frac{\partial^{2} \omega}{\partial t^{2}}+(\delta \mathrm{d}+\mathrm{d} \delta) \omega=g
$$

If we assume that $g$ is time-harmonic, i.e., can be expressed as the product of a function which is independent of time with $e^{\mathrm{i} m t}$ for some positive real number $m$, and then we seek $\omega$ of the same form, we are led to a timeindependent equation of the form

$$
(\delta \mathrm{d}+\mathrm{d} \delta) u-m^{2} u=f .
$$

With the boundary conditions $\operatorname{Tr}(\star u)=0$ and $\operatorname{Tr}(\star \mathrm{d} u)=0$, a weak formulation of this problem is as follows.

Find $u \in H \Lambda^{k}(\Omega)$ such that

$$
\langle\mathrm{d} u, \mathrm{~d} v\rangle+\langle\delta u, \delta v\rangle-m^{2}\langle u, v\rangle=\langle f, v\rangle, \quad v \in H \Lambda^{k}(\Omega) .
$$

If we consider the special case $f \in \mathfrak{B}^{* k}$, and split $u$ by its Hodge decomposition, $u=u_{\mathrm{d}}+u_{\mathfrak{H}}+u_{\delta}$, then the subproblem for $u_{\delta}$ may be written:

Find $u \in \mathfrak{B}^{* k}$ such that

$$
\langle\mathrm{d} u, \mathrm{~d} v\rangle-m^{2}\langle u, v\rangle=\langle f, v\rangle, \quad v \in \mathfrak{B}^{* k} .
$$

Note that for $f \in \mathfrak{B}^{* k}$, this problem is equivalent to (9.4) (in the sense that the unique solution to this problem is the unique solution of (9.4)).

To approximate (9.4), we use the obvious extension of the method defined in (9.2). That is, we seek $u_{h} \in \Lambda_{h}^{k}$ such that

$$
\left\langle\mathrm{d} u_{h}, \mathrm{~d} v\right\rangle-m^{2}\left\langle u_{h}, v\right\rangle=\langle f, v\rangle, \quad v \in \Lambda_{h}^{k}
$$

Note that since we have assumed that $m^{2}$ is not an eigenvalue for the continuous problem (9.4), it follows from Theorem 8.4 that $m^{2}$ is not an eigenvalue for the corresponding discrete problem (9.5) if the mesh parameter $h$ is sufficiently small. In fact the following error bound can be established.

Theorem 9.1. Assume that $f \in L^{2} \Lambda^{k}(\Omega)$ and a real number $m>0$ are fixed. Furthermore, let $u \in H \Lambda^{k}(\Omega)$ and $u_{h} \in \Lambda_{h}^{k}$ be the corresponding solutions of (9.4) and (9.5), respectively. If the domain $\Omega$ is $s$-regular, with $0<s \leq 1$, then for $h$ sufficiently small,

$$
\left\|u-u_{h}\right\|_{H \Lambda} \leq \frac{1}{1-C h^{s}} \inf _{v \in \Lambda_{h}^{k}}\|u-v\|_{H \Lambda}
$$

where the constant $C$ is independent of $h$.

Proof. To obtain this result, we follow the proof of Monk (2003b), but 
for the case of natural, rather than essential boundary conditions; see also Monk (1992), Monk and Demkowicz (2001), and Boffi and Gastaldi (2002). First, subtracting (9.5) from (9.4), and setting $e_{h}=u-u_{h}$, we obtain the error equation

$$
\left\langle\mathrm{d} e_{h}, \mathrm{~d} v\right\rangle-m^{2}\left\langle e_{h}, v\right\rangle=0, \quad v \in \Lambda_{h}^{k} .
$$

If we choose $v=\mathrm{d} q$ for $q \in \Lambda_{h}^{k-1}$, we get

$$
\left\langle e_{h}, \mathrm{~d} q\right\rangle=0, \quad q \in \Lambda_{h}^{k-1} .
$$

Letting $Q_{h}$ denote the $H \Lambda$-projection into $\Lambda_{h}^{k}$, we then obtain from (9.6)

$$
\begin{aligned}
\left\|e_{h}\right\|_{H \Lambda}^{2} & =\left\langle\mathrm{d} e_{h}, \mathrm{~d} e_{h}\right\rangle-m^{2}\left\langle e_{h}, e_{h}\right\rangle+\left(1+m^{2}\right)\left\langle e_{h}, e_{h}\right\rangle \\
& =\left\langle\mathrm{d} e_{h}, \mathrm{~d}\left(u-Q_{h} u\right)\right\rangle-m^{2}\left\langle e_{h}, u-Q_{h} u\right\rangle+\left(1+m^{2}\right)\left\langle e_{h}, e_{h}\right\rangle \\
& =\left\langle\mathrm{d} e_{h}, \mathrm{~d}\left(u-Q_{h} u\right)\right\rangle+\left\langle e_{h}, u-Q_{h} u\right\rangle+\left(1+m^{2}\right)\left\langle e_{h}, Q_{h} u-u_{h}\right\rangle \\
& =\left\langle\mathrm{d} e_{h}, \mathrm{~d}\left(u-Q_{h} u\right)\right\rangle+\left\langle e_{h}, u-Q_{h} u\right\rangle+\left(1+m^{2}\right)\left\langle e_{h}, Q_{h} e_{h}\right\rangle \\
& \leq\left\|e_{h}\right\|_{H \Lambda}\left\|u-Q_{h} u\right\|_{H \Lambda}+\left(1+m^{2}\right)\left\langle e_{h}, Q_{h} e_{h}\right\rangle .
\end{aligned}
$$

The main work of the proof is then to estimate the term $\left\langle e_{h}, Q_{h} e_{h}\right\rangle$. To do so, we use the Hodge decomposition to write

$$
e_{h}=\mathrm{d} \rho+\psi, \quad \rho \in H \Lambda^{k-1}, \psi \in \mathfrak{B}^{k \perp}, \quad\left\|e_{h}\right\|^{2}=\|\mathrm{d} \rho\|^{2}+\|\psi\|^{2},
$$

and the discrete Hodge decomposition to write

$$
Q_{h} e_{h}=\mathrm{d} \rho_{h}+\psi_{h}, \quad \rho_{h} \in \Lambda_{h}^{k-1}, \quad \psi_{h} \in \mathfrak{B}_{h}^{k \perp}, \quad\left\|Q_{h} e_{h}\right\|^{2}=\left\|\mathrm{d} \rho_{h}\right\|^{2}+\left\|\psi_{h}\right\|^{2} .
$$

Then by (9.7),

$$
\left\langle e_{h}, Q_{h} e_{h}\right\rangle=\left\langle e_{h}, \mathrm{~d} \rho_{h}+\psi_{h}\right\rangle=\left\langle e_{h}, \psi_{h}\right\rangle=\left\langle\mathrm{d} \rho, \psi_{h}\right\rangle+\left\langle\psi, \psi_{h}\right\rangle .
$$

We next obtain a bound on $\|\psi\|_{0}$. To do so, we define the adjoint variable $z \in H \Lambda^{k}(\Omega)$ satisfying

$$
\langle\mathrm{d} \phi, \mathrm{d} z\rangle-m^{2}\langle z, \phi\rangle=\langle\psi, \phi\rangle, \quad \phi \in H \Lambda^{k}(\Omega) .
$$

Since $m$ is not an eigenvalue, $z$ is well defined and there is a constant $C$ such that $\|z\|_{H \Lambda} \leq C\|\psi\|$. Furthermore, since $\langle\mathrm{d} q, \psi\rangle=0$ for $q \in \Lambda^{k-1}$, it follows that $\langle\mathrm{d} q, \bar{z}\rangle=0$ for for $q \in \Lambda^{k-1}$. Hence, $\delta z=0$. Since $\operatorname{Tr} \star z=0$ and $\Omega$ is $s$-regular, $z \in H^{s}(\Omega)$ for some $s \in(0,1]$ and from $(7.24)$, we have the estimate $\|z\|_{H^{s}} \leq C\|\mathrm{~d} z\| \leq C\|\psi\|$. In addition, we see that $d z$ satisfies

$$
\delta \mathrm{d} z=m^{2} z+\psi, \quad \operatorname{Tr} \star \mathrm{d} z=0 .
$$

Again using (7.24), we obtain $d z \in H^{s}(\Omega)$ and

$$
\|\mathrm{d} z\|_{H^{s}} \leq C\|\delta \mathrm{d} z\|_{0} \leq C\left\|m^{2} z+\psi\right\| \leq C\|\psi\| .
$$


Using the approximation properties of $\tilde{\Pi}_{h}$, we obtain the estimate

$$
\left\|z-\tilde{\Pi}_{h} z\right\|_{H \Lambda} \leq C h^{s}\left(\|z\|_{s}+\|\mathrm{d} z\|_{s}\right) \leq C h^{s}\|\psi\| .
$$

Choosing $\phi=\psi$ in (9.10), and using the fact that $\delta z=0$, and (9.6), we get

$$
\begin{aligned}
\|\psi\|^{2} & =\langle\mathrm{d} \psi, \mathrm{d} z\rangle-m^{2}\langle z, \psi\rangle=\left\langle\mathrm{d} e_{h}, \mathrm{~d} z\right\rangle-m^{2}\left\langle z, e_{h}\right\rangle \\
& =\left\langle\mathrm{d} e_{h}, \mathrm{~d}\left(z-\tilde{\Pi}_{h} z\right)\right\rangle-m^{2}\left\langle z-\tilde{\Pi}_{h} z, e_{h}\right\rangle \\
& \leq C\left\|e_{h}\right\|_{H \Lambda}\left\|z-\tilde{\Pi}_{h} z\right\|_{H \Lambda} \leq C h^{s}\left\|e_{h}\right\|_{H \Lambda}\|\psi\| .
\end{aligned}
$$

Hence, $\|\psi\| \leq C h^{s}\left\|e_{h}\right\|_{H \Lambda}$, and so

$$
\left|\left\langle\psi, \psi_{h}\right\rangle\right| \leq\|\psi\|\left\|\psi_{h}\right\| \leq\|\psi\|\left\|Q_{h} e_{h}\right\| \leq C h^{s}\left\|e_{h}\right\|_{H \Lambda}^{2} .
$$

It thus remains to bound the term $\left\langle\mathrm{d} \rho, \psi_{h}\right\rangle$. To do so, we first write $\psi_{h}=\psi_{h, \delta}+\psi_{h, \mathfrak{H}}$, with $\psi_{h, \delta} \in \mathfrak{Z}_{h}^{k \perp}$, and $\psi_{h, \mathfrak{H}} \in \mathfrak{H}_{h}^{k}$. Then, by Lemmas 5.10 and 5.9 , we can find $\chi_{\delta} \in \mathfrak{Z}^{k \perp}$ and and $\chi_{\mathfrak{H}} \in \mathfrak{H}^{k}$ satisfying $d \chi_{\delta}=\mathrm{d} \psi_{h}$, $\left\|\chi_{\mathfrak{H}}\right\| \leq\left\|\psi_{h, \mathfrak{H}}\right\|$ and (also using (7.24)),

$$
\begin{aligned}
& \left\|\psi_{h, \delta}-\chi_{\delta}\right\| \leq\left\|\left(I-\tilde{\Pi}_{h}\right) \chi_{\delta}\right\| \leq C h^{s}\left\|\chi_{\delta}\right\|_{s} \leq C h^{s}\left\|\mathrm{~d} \chi_{\delta}\right\| \leq C h^{s}\left\|\mathrm{~d} \psi_{h}\right\|, \\
& \left\|\psi_{h, \mathfrak{H}}-\chi_{\mathfrak{H}}\right\| \leq\left\|\left(I-\tilde{\Pi}_{h}\right) \chi_{\mathfrak{H}}\right\| \leq C h^{s}\left\|\chi_{\mathfrak{H}}\right\|_{s} \leq C h^{s}\left\|\chi_{\mathfrak{H}}\right\| \leq C h^{s}\left\|\psi_{h, \mathfrak{H}}\right\| .
\end{aligned}
$$

Hence,

$$
\begin{aligned}
\left|\left\langle\mathrm{d} \rho, \psi_{h}\right\rangle\right| & =\left|\left\langle\mathrm{d} \rho, \psi_{h, \delta}-\chi_{\delta}+\psi_{h, \mathfrak{H}}-\chi_{\mathfrak{H}}\right\rangle\right| \\
& \leq C h^{s}\|\mathrm{~d} \rho\|\left(\left\|\mathrm{d} \psi_{h}\right\|+\left\|\psi_{h, \mathfrak{H}}\right\|\right) \leq C h^{s}\|\mathrm{~d} \rho\|\left(\left\|\mathrm{d} \psi_{h}\right\|+\left\|\psi_{h}\right\|\right) \\
& \leq C h^{s}\left\|e_{h}\right\|\left\|Q_{h} e_{h}\right\|_{H \Lambda} \leq C h^{s}\left\|e_{h}\right\|_{H \Lambda}^{2} .
\end{aligned}
$$

Combining (9.8), (9.9), (9.11), and (9.12), we obtain

$$
\left\|e_{h}\right\|_{H \Lambda}^{2} \leq\left\|e_{h}\right\|_{H \Lambda}\left\|u-Q_{h} u\right\|_{H \Lambda}+\left(1+m^{2}\right) C h^{s}\left\|e_{h}\right\|_{H \Lambda}^{2} .
$$

The theorem follows immediately from this result, provided $h$ is sufficiently small.

\subsection{Refined estimates}

In the error estimates derived above, the estimates for $\left\|u-u_{h}\right\|$ and $\| \mathrm{d}(u-$ $\left.u_{h}\right) \|$ are tied together in the $H \Lambda$-norm. It is well known, however, that for the standard Galerkin method (corresponding to the case $k=0$ ), the error bounds for $\left\|u-u_{h}\right\|$ can be up to one power higher in $h$ than the error for $\left\|\mathrm{d}\left(u-u_{h}\right)\right\|$.

To understand the type of improvement we might hope to get in this more general case, we use the Hodge decomposition to write the error $u-u_{h}=$ $e_{\mathrm{d}}+e_{\mathfrak{H}}+e_{\delta}=\mathrm{d} g+e_{\mathfrak{H}}+e_{\delta}$, where $e_{\mathrm{d}} \in \mathfrak{B}^{k}, e_{\mathfrak{H}} \in \mathfrak{H}^{k}, e_{\delta} \in \stackrel{\circ}{\mathfrak{B}}^{* k}$, and $g \in \stackrel{\circ}{B}^{*(k-1)}$. Since $\left\|e_{\mathrm{d}}+e_{\mathfrak{H}}\right\|_{H \Lambda}=\left\|e_{\mathrm{d}}+e_{\mathfrak{H}}\right\|$, we cannot expect to improve on this part of the error. However, we will establish the following result. 
Theorem 9.2. Suppose $u \in H \Lambda^{k}(\Omega)$ is the solution of problem (9.1) and $u_{h} \in \Lambda_{h}^{k}$ is the solution of problem (9.2). Further suppose that the domain $\Omega$ is 1-regular and that $u-u_{h}=\mathrm{d} g+e_{\mathfrak{H}}+e_{\delta}$, where $g \in \mathfrak{B}^{*(k-1)}, e_{\mathfrak{H}} \in \mathfrak{H}^{k}$, and $e_{\delta} \in \mathfrak{B}^{* k}$. Then there is a constant C independent of $h$ such that

$$
\|g\| \leq C h\left\|u-u_{h}\right\|, \quad\left\|e_{\delta}\right\| \leq C h\left\|u-u_{h}\right\|_{H \Lambda} .
$$

Proof. To obtain the first bound, we observe that by (9.3)

$$
\left\langle\mathrm{d} g, \mathrm{~d} z_{h}\right\rangle=\left\langle u-u_{h}, \mathrm{~d} z_{h}\right\rangle=0, \quad z_{h} \in \Lambda_{h}^{k-1} .
$$

Since $g \in \mathfrak{B}^{*(k-1)}$, we can define (see (7.3)) $z^{g} \in \mathfrak{\mathfrak { B }}^{*(k-1)}$ such that

$$
\left\langle\mathrm{d} z^{g}, \mathrm{~d} \mu\right\rangle=\langle g, \mu\rangle, \quad \mu \in H \Lambda^{k-1}(\Omega) .
$$

Hence, choosing $\mu=g$, we obtain

$$
\|g\|^{2}=\left\langle\mathrm{d} z^{g}, \mathrm{~d} g\right\rangle=\left\langle\mathrm{d}\left(z^{g}-z_{h}\right), \mathrm{d} g\right\rangle \leq\left\|\mathrm{d}\left(z^{g}-z_{h}\right)\right\|\left\|u-u_{h}\right\| .
$$

Choosing $z_{h}=\tilde{\Pi}_{h} z^{g}$, using (7.24), and noting that $\delta \mathrm{d} z^{g}=g$ and $\operatorname{Tr} \star \mathrm{d} z^{g}=$ 0 , we get that

$$
\left\|\mathrm{d}\left(z^{g}-z_{h}\right)\right\|=\left\|\left(I-\tilde{\Pi}_{h}\right) \mathrm{d} z^{g}\right\| \leq C h\left\|\mathrm{~d} z^{g}\right\|_{1} \leq C h\left\|\delta \mathrm{d} z^{g}\right\| \leq C h\|g\| .
$$

This establishes the first estimate. To get the second estimate, we now define $z^{\delta} \in \mathfrak{B}^{* k}$ such that

$$
\left\langle z^{\delta}, \mu\right\rangle_{H \Lambda}=\left\langle e_{\delta}, \mu\right\rangle, \quad \mu \in H \Lambda^{k}(\Omega) .
$$

Then using (9.3), we get

$$
\begin{aligned}
\left\|e_{\delta}\right\|^{2} & =\left\langle e_{\delta}, u-u_{h}\right\rangle=\left\langle z^{\delta}, u-u_{h}\right\rangle_{H \Lambda} \\
& =\left\langle z^{\delta}-\tilde{\Pi}_{h} z^{\delta}, u-u_{h}\right\rangle_{H \Lambda} \leq\left\|z^{\delta}-\tilde{\Pi}_{h} z^{\delta}\right\|_{H \Lambda}\left\|u-u_{h}\right\|_{H \Lambda} \\
& \leq C h\left(\left\|z^{\delta}\right\|_{1}+\left\|\mathrm{d} z^{\delta}\right\|_{1}\right)\left\|u-u_{h}\right\|_{H \Lambda} .
\end{aligned}
$$

Again applying (7.24), we find that

$$
\begin{gathered}
\left\|z^{\delta}\right\|_{1} \leq C\left\|\mathrm{~d} z^{\delta}\right\| \leq C\left\|e_{\delta}\right\| \\
\left\|\mathrm{d} z^{\delta}\right\|_{1} \leq C\left\|\delta \mathrm{d} z^{\delta}\right\| \leq C\left(\left\|e_{\delta}\right\|+\left\|z^{\delta}\right\|\right) \leq C\left\|e_{\delta}\right\| .
\end{gathered}
$$

The result follows by combining these estimates.

We close by remarking that essentially the same result holds, with essentially the same proof, in the case where $u$ is the solution of (9.4) and $u_{h}$ is defined by (9.5).

\section{Preconditioning}

The purpose of this section is to discuss preconditioned iterative methods for the discrete Hodge-Laplace problem. Such solvers have been considered previously for some of the PDE problems that can be derived from the 
Hodge-Laplace problem, but to our knowledge not in the full generality presented here. We will first give a quick review of Krylov space iterations and block diagonal preconditioners. Thereafter, we will illustrate how the discrete Hodge decomposition enters as a fundamental tool in the construction of the diagonal blocks of the preconditioner.

The discrete Hodge-Laplace problem can be written as a linear system

$$
\mathcal{A}_{h} x_{h}=f_{h},
$$

where the operator $\mathcal{A}_{h}$ is a self adjoint operator from $X_{h}=\Lambda_{h}^{k-1} \times \Lambda_{h}^{k}$ onto its dual $X_{h}^{*}$ and $f_{h} \in X_{h}^{*}$ is given. In the discussion below, we will consider $X_{h}^{*}=X_{h}$ as a set, but with different norms and inner products.

If systems of the form (10.1) are solved by an iterative method, then the convergence of the iteration depends on the conditioning of the coefficient operator $\mathcal{A}_{h}$. In particular, the convergence rate can be bounded by the spectral condition number of the operator $\mathcal{A}_{h}, \operatorname{cond}\left(\mathcal{A}_{h}\right)$, given by

$$
\operatorname{cond}\left(\mathcal{A}_{h}\right)=\frac{\sup |\lambda|}{\inf |\lambda|},
$$

where the supremum and infimum are taken over the spectrum of $\mathcal{A}_{h}$. For linear operators arising as discretization of elliptic operators such as the Hodge Laplacian, this condition number will typically tend to infinity as the mesh parameter $h$ tends to zero, leading to slow convergence of the iterative methods for fine triangulations $\mathcal{T}_{h}$. The standard way to overcome this problem is to introduce preconditioners.

\subsection{Krylov space iterations}

Consider a linear equation of the form

$$
\mathcal{A} x=f,
$$

where $f \in X$ is given, $x \in X$ is the unknown, and $\mathcal{A} \in \mathcal{L}(X, X)$ is a symmetric operator mapping a Hilbert space $X$ into itself. Furthermore, assume that $\mathcal{A}$ is invertible with $\mathcal{A}^{-1} \in \mathcal{L}(X, X)$. Solutions of equations of the form (10.3) can be approximated by a Krylov space iteration. For each integer $m \geq 1$ we define $K_{m}=K_{m}(f)$ as the finite-dimensional subspace of $X$ spanned by the elements $f, \mathcal{A} f, \ldots, \mathcal{A}^{m-1} f$. If $\mathcal{A}$ is positive definite then the approximations $x_{m} \in K_{m}$ can be generated by the conjugate gradient method, i.e. $x_{m}$ is uniquely determined by

$$
\left\langle\mathcal{A} x_{m}, y\right\rangle_{X}=\langle f, y\rangle_{X}, \quad y \in X
$$

or, equivalently, $x_{m}$ is the minimizer of the quadratic functional

$$
F(y)=\frac{1}{2}\langle\mathcal{A} y, y\rangle_{X}-\langle f, y\rangle_{X}
$$


over $K_{m}$. Here, $\langle\cdot, \cdot\rangle_{X}$ is the inner product in $X$, and $\|\cdot\|_{X}$ is the corresponding norm. If the coefficient operator $\mathcal{A}$ is indefinite, we can instead use the minimum residual method, where $x_{m} \in K_{m}$ is uniquely characterized by

$$
\left\|\mathcal{A} x_{m}-f\right\|_{X}=\inf _{y \in X}\|\mathcal{A} y-f\|_{X}
$$

These optimal characterizations of the approximations $x_{m}$ ensure that the iterative method converges and that the reduction factor

$$
\frac{\left\|x_{m}-x\right\|_{X}}{\left\|x_{0}-x\right\|_{X}}
$$

can be bounded, a priori, by a function only depending on the number of iterations, $m$, and the condition number of the coefficient operator $\mathcal{A}$ given by

$$
\operatorname{cond}(\mathcal{A})=\|\mathcal{A}\|_{\mathcal{L}(X, X)} \cdot\left\|\mathcal{A}^{-1}\right\|_{\mathcal{L}(X, X)} \cdot
$$

However, from a computational point of view, it is also important that the approximations $x_{m}$ can be cheaply computed by a recurrence relation. Typically, only one or two evaluations of the operator $\mathcal{A}$, in addition to a few calculations of the inner products $\langle\cdot, \cdot\rangle_{X}$, are required to compute $x_{m}$ from $x_{m-1}$. For more details on Krylov space methods, we refer, for example, to Hackbusch (1994).

\subsection{Block diagonal preconditioners}

Our goal is to design effective iterations for the discrete Hodge-Laplace problem. However, we will motivate the approach by first studying the corresponding continuous problem. For simplicity we will assume throughout this subsection that there are no nontrivial harmonic $k$-forms, i.e., $\mathfrak{H}^{k}=\{0\}$, since the inclusion of such a finite-dimensional space of multipliers is relatively insignificant for the design of preconditioners. Furthermore, the boundary conditions are taken to be $\operatorname{Tr} \star u=0$ and $\operatorname{Tr} \star \mathrm{d} u=0$.

The continuous problem

Recall that the Hodge-Laplace problem in mixed form is as follows.

Find $(\sigma, u) \in H \Lambda^{k-1}(\Omega) \times H \Lambda^{k}(\Omega)$ such that

$$
\begin{aligned}
\langle\sigma, \tau\rangle-\langle\mathrm{d} \tau, u\rangle & =0, & \tau \in H \Lambda^{k-1}(\Omega), \\
\langle\mathrm{d} \sigma, v\rangle+\langle\mathrm{d} u, \mathrm{~d} v\rangle & =\langle f, v\rangle, & v \in H \Lambda^{k}(\Omega),
\end{aligned}
$$

where $f \in L^{2} \Lambda^{k}(\Omega)$ is given. In order to align the description of this system with the framework above, we let $X=H \Lambda^{k-1}(\Omega) \times H \Lambda^{k}(\Omega)$, and $H=$ $L^{2} \Lambda^{k-1}(\Omega) \times L^{2} \Lambda^{k}(\Omega)$. Note that any element $g=\left(g^{k-1}, g^{k}\right) \in H$ gives rise to an element of $X^{*}$ by the identification

$$
x=(\tau, v) \mapsto\langle g, x\rangle=\left\langle g^{k-1}, \tau\right\rangle+\left\langle g^{k}, v\right\rangle, \quad x=(\tau, v) \in X .
$$


In fact, $X^{*}$ can be identified with the completion of $H$ in the dual norm

$$
\|g\|_{X^{*}}=\sup _{x \in X,\|x\|_{X}=1}\langle g, x\rangle .
$$

In this way we obtain a Gelfand triple

$$
X \subset H \subset X^{*},
$$

where $X$ is dense in $H$ and $H$ is dense in $X^{*}$. Furthermore, the duality pairing between $X^{*}$ and $X$ can be defined as an extension of the $H$-inner product, still denoted $\langle\cdot, \cdot\rangle$.

The system (10.4) can now be formally written in the form

$$
\mathcal{A} x=f,
$$

where $x=(\sigma,-u) \in X, f \in X^{*}$ is the functional associated to $(0, f) \in H$ and $\mathcal{A}: X \rightarrow X^{*}$ is the operator

$$
\mathcal{A}=\left(\begin{array}{cc}
I & \mathrm{~d}^{*} \\
\mathrm{~d} & -\mathrm{d}^{*} \mathrm{~d}
\end{array}\right) .
$$

Here $\mathrm{d}^{*}=\mathrm{d}_{k}^{*}: L^{2} \Lambda^{k+1}(\Omega) \rightarrow\left(H \Lambda^{k}(\Omega)\right)^{*}$ is the adjoint of $\mathrm{d}_{k}: H \Lambda^{k}(\Omega) \rightarrow$ $L^{2} \Lambda^{k+1}(\Omega)$, i.e.,

$$
\left\langle\mathrm{d}^{*} \tau, \omega\right\rangle=\langle\tau, \omega \mathrm{d}\rangle, \quad \tau \in L^{2} \Lambda^{k+1}(\Omega), \omega \in H \Lambda^{k}(\Omega)
$$

where, as above, $\langle\cdot, \cdot\rangle$ is the extension of the inner product on $L^{2} \Lambda^{k}(\Omega)$ to the duality pairing between $\left(H \Lambda^{k}(\Omega)\right)^{*}$ and $H \Lambda^{k}(\Omega)$.

Note that the operator $\mathcal{A}$ is $H$-symmetric in the sense that if $x=(\sigma, u) \in$ $X$ and $y=(\tau, v) \in X$, then

$$
\langle\mathcal{A} x, y\rangle=\langle\sigma, \tau\rangle+\langle\mathrm{d} \sigma, v\rangle+\langle\mathrm{d} \tau, u\rangle-\langle\mathrm{d} u, \mathrm{~d} v\rangle=\langle x, \mathcal{A} y\rangle .
$$

On the other hand, the operator $\mathcal{A}$ is not positive definite in any sense.

It is a consequence of the discussion Section 7 (see Theorem 7.2), that $\mathcal{A}$ defines an isomorphism between $X$ and $X^{*}$, i.e.

$$
\mathcal{A} \in \mathcal{L}\left(X, X^{*}\right) \quad \text { and } \mathcal{A}^{-1} \in \mathcal{L}\left(X^{*}, X\right) .
$$

However, since the operator $\mathcal{A}$ maps the Hilbert space $X$ into a larger space $X^{*}$, a Krylov space iteration for the linear system (10.5) is not well-defined. Instead we will consider an equivalent preconditioned system of the form

$$
\mathcal{B} \mathcal{A} x=\mathcal{B} f,
$$

where $\mathcal{B} \in \mathcal{L}\left(X^{*}, X\right)$ is an isomorphism, i.e., we also have $\mathcal{B}^{-1} \in \mathcal{L}\left(X, X^{*}\right)$. Hence, the new coefficient operator $\mathcal{B A}$ is an element of $\mathcal{L}(X, X)$ with a bounded inverse. In order to preserve the symmetry of the system we will also require that $\mathcal{B}$ is symmetric in the sense that

$$
\langle\mathcal{B} f, g\rangle=\langle f, \mathcal{B} g\rangle,
$$


and positive definite in the sense that there is a constant $C>0$ such that

$$
\langle\mathcal{B} f, f\rangle \geq C\|\mathcal{B} f\|_{X}, \quad f \in X^{*}
$$

If these conditions are satisfied, then the bilinear form $\left\langle\mathcal{B}^{-1} \cdot, \cdot\right\rangle$ defines a new inner product on $X$, and the coefficient operator $\mathcal{B A}$ of $(10.6)$ is symmetric in this inner product since

$$
\left\langle\mathcal{B}^{-1} \mathcal{B} \mathcal{A} x, y\right\rangle=\langle\mathcal{A} x, y\rangle=\left\langle\mathcal{B}^{-1} x, \mathcal{B} \mathcal{A} y\right\rangle, \quad x, y \in X .
$$

Therefore the preconditioned system (10.6) fulfils all the properties required such that the minimum residual can be applied. But observe that in order to do so, the preconditioner $\mathcal{B}$ has to be evaluated at least once for each iteration.

One possible operator $\mathcal{B}$ with all the required properties for a preconditioner is the block diagonal operator operator given by

$$
\left(\begin{array}{cc}
\left(I+\mathrm{d}^{*} \mathrm{~d}\right)^{-1} & 0 \\
0 & \left(I+\mathrm{d}^{*} \mathrm{~d}\right)^{-1}
\end{array}\right)=\left(\begin{array}{cc}
\left(I+\mathrm{d}_{k-1}^{*} \mathrm{~d}_{k-1}\right)^{-1} & 0 \\
0 & \left(I+\mathrm{d}_{k}^{*} \mathrm{~d}_{k}\right)^{-1}
\end{array}\right) .
$$

Here the operator $\left(I+\mathrm{d}^{*} \mathrm{~d}\right)^{-1} \in \mathcal{L}\left(\left(H \Lambda^{k}(\Omega)\right)^{*}, H \Lambda^{k}(\Omega)\right)$ is the solution operator defined from the inner product on $H \Lambda^{k}(\Omega)$, i.e., $\left(I+\mathrm{d}^{*} \mathrm{~d}\right)^{-1} f=\omega$ if

$$
\langle\omega, \tau\rangle_{H \Lambda}=\langle f, \tau\rangle, \quad \tau \in H \Lambda^{k}(\Omega) .
$$

Furthermore, any block diagonal operator of the form

$$
\mathcal{B}=\left(\begin{array}{cc}
B_{k-1} & 0 \\
0 & B_{k}
\end{array}\right)
$$

where $B=B_{k} \in \mathcal{L}\left(H \Lambda^{k}(\Omega)^{*}, H \Lambda^{k}(\Omega)\right)$ is a preconditioner for the operator $I+\mathrm{d}^{*} \mathrm{~d}$ will also work. Here an operator $B \in \mathcal{L}\left(H \Lambda^{k}(\Omega)^{*}, H \Lambda^{k}(\Omega)\right)$ is referred to as a preconditioner for $I+\mathrm{d}^{*} \mathrm{~d}$ if it is an isomorphism which is symmetric and positive definite with respect to $\langle\cdot, \cdot\rangle$.

\section{The discrete problem}

The main reason for including the discussion of preconditioners for the continuous problem above was to use it to motivate the corresponding discussion for the discrete case. Of course, in real computations we need to apply preconditioners for the discrete problem. In fact, there exist several approaches to the design of preconditioners for mixed problems such as the discrete Hodge-Laplace problem, and not all of them are of the form discussed here. As an example, we mention, the positive definite reformulation, performed in Bramble and Pasciak (1988), and the use of triangular preconditioners, as discussed in Klawonn (1998). However, here, in complete analogy with the discussion above, we restrict the attention to block diagonal preconditioners, as discussed in Rusten and Winther (1992), Silvester 
and Wathen (1994), Arnold, Falk and Winther (1997a), and Bramble and Pasciak (1997), for example.

Recall that the discrete Hodge-Laplace problem takes the following form. For a given $f \in L^{2} \Lambda^{k}(\Omega)$, find $\left(\sigma_{h}, u_{h}\right) \in \Lambda_{h}^{k-1} \times \Lambda_{h}^{k}$ such that

$$
\begin{aligned}
\left\langle\sigma_{h}, \tau\right\rangle-\left\langle\mathrm{d} \tau, u_{h}\right\rangle & =0, & \tau \in \Lambda_{h}^{k-1}, \\
\left\langle\mathrm{~d} \sigma_{h}, v\right\rangle+\left\langle\mathrm{d} u_{h}, \mathrm{~d} v\right\rangle & =\langle f, v\rangle, & v \in \Lambda_{h}^{k} .
\end{aligned}
$$

We assume throughout this section that the discrete spaces $\Lambda_{h}^{k-1}$ and $\Lambda_{h}^{k}$ are chosen such that they are part of one of the discrete de Rham complexes introduced in $\S 5$ above.

Let $X_{h}=\Lambda_{h}^{k-1} \times \Lambda_{h}^{k}$ and define an operator $\mathcal{A}_{h}: X_{h} \rightarrow X_{h}^{*}$ by

$$
\left\langle\mathcal{A}_{h} x, y\right\rangle=\langle\sigma, \tau\rangle+\langle\mathrm{d} \sigma, v\rangle+\langle\mathrm{d} \tau, u\rangle-\langle\mathrm{d} u, \mathrm{~d} v\rangle
$$

where $x=(\sigma, u)$ and $y=(\tau, v)$, and, as above, $\langle\cdot, \cdot\rangle$ is the inner product on $H=L^{2} \Lambda^{k-1}(\Omega) \times L^{2} \Lambda^{k}(\Omega)$. The space $X_{h}^{*}$ will be equal to $X_{h}$ as a set, but with norm given by

$$
\|g\|_{X_{h}^{*}}=\sup _{x \in X_{h},\|x\|_{X}=1}\langle g, x\rangle .
$$

With these definitions, the system (10.7) can be alternatively written as

$$
\mathcal{A}_{h} x_{h}=f_{h},
$$

where $x_{h}=\left(\sigma_{h},-u_{h}\right)$ and where $f_{h} \in X_{h}^{*}$ represents the data.

Under the present conditions, the operator $\mathcal{A}_{h}$ is invertible and symmetric with respect to the inner product $\langle\cdot, \cdot\rangle$. Furthermore, since $\mathcal{A}_{h}$ maps $X_{h}$ into itself, a Krylov space iteration, like the minimum residual method, is well-defined for the system (10.9). On the other hand, it can be shown that the spectral condition number of $\mathcal{A}_{h}$, defined by (10.2), will grow asymptotically like $h^{-2}$ as the mesh parameter $h$ tends to zero. Therefore, such an iteration will not be very efficient for fine triangulations, and it is also necessary in the discrete case to introduce a preconditioner in order to obtain an efficient iteration.

If we let $X_{h}$ inherit the norm of $X=H \Lambda^{k-1}(\Omega) \times H \Lambda^{k}(\Omega)$, and the norm of $X_{h}^{*}$ is given by (10.8), then it follows from results of Section 7 (see Theorem 7.3), that the norms

$$
\left\|\mathcal{A}_{h}\right\|_{\mathcal{L}\left(X_{h}, X_{h}^{*}\right)} \quad \text { and } \quad\left\|\mathcal{A}_{h}^{-1}\right\|_{\mathcal{L}\left(X_{h}^{*}, X_{h}\right)}
$$

are both bounded independently of the mesh parameter $h$. Therefore, if $\mathcal{B}_{h}$ is another operator on $X_{h}$ such that the norms

$$
\left\|\mathcal{B}_{h}\right\|_{\mathcal{L}\left(X_{h}^{*}, X_{h}\right)} \quad \text { and } \quad\left\|\mathcal{B}_{h}^{-1}\right\|_{\mathcal{L}\left(X_{h}, X_{h}^{*}\right)}
$$

are bounded independently of $h$, then the spectral condition number of the composition $\mathcal{B}_{h} \mathcal{A}_{h}$ is bounded independently of $h$. As above, we can also 
argue that if the preconditioner $\mathcal{B}_{h}$ is symmetric and positive definite with respect to the inner product $\langle\cdot, \cdot\rangle$, then the operator $\mathcal{B}_{h} \mathcal{A}_{h}$ is symmetric with respect to the inner product $\left\langle\mathcal{B}_{h}^{-1} \cdot, \cdot\right\rangle$ on $X_{h}$. Hence, the preconditioned system

$$
\mathcal{B}_{h} \mathcal{A}_{h} x_{h}=\mathcal{B}_{h} f_{h}
$$

fulfils all the necessary conditions required for applying the minimum residual method. Furthermore, since the condition numbers of the operators $\mathcal{B}_{h} \mathcal{A}_{h}$ are bounded independently of $h$, the convergence rate will not deteriorate as the mesh becomes finer.

As in the continuous case, it seems canonical to construct block diagonal preconditioners of the form

$$
\mathcal{B}_{h}=\left(\begin{array}{cc}
B_{k-1, h} & 0 \\
0 & B_{k, h}
\end{array}\right),
$$

where the operator $B_{h}=B_{k, h}: \Lambda_{h}^{k} \rightarrow \Lambda_{h}^{k}$ is constructed such that it is symmetric with respect to the $L^{2}$-inner product $\langle\cdot, \cdot\rangle$, and such that there exists constants $c_{1}, c_{2}>0$, independent of $h$, satisfying

$$
c_{1}\left\langle B_{h}^{-1} \omega, \omega\right\rangle \leq\langle\omega, \omega\rangle_{H \Lambda} \leq c_{2}\left\langle B_{h}^{-1} \omega, \omega\right\rangle \quad \omega \in \Lambda_{h}^{k}
$$

If both operators $B_{k-1, h}$ and $B_{k, h}$ occurring in (10.11) satisfy such a spectral equivalence relation, the corresponding operator $\mathcal{B}_{h}$ will satisfy all requirements given above. In particular, the norms given in (10.10) will be bounded independently of $h$.

Finally, we observe that the preconditioner $\mathcal{B}_{h}$, and hence the operators $B_{k-1, h}$ and $B_{k, h}$, have to be evaluated at least once for each iteration. Therefore, the operators $B_{k-1, h}$ and $B_{k, h}$ have to be defined such that they can be evaluated effectively. The real challenge is to design preconditioners $B_{h}$ which satisfy (10.12) and which, at the same time, can be evaluated cheaply. The construction of the operators $B_{h}$ will be further discussed below.

\subsection{Constructing effective preconditioners}

Motivated by the development above, we will now discuss the design of preconditioners for the discrete operators $I+\mathrm{d}_{h}^{*} \mathrm{~d}_{h}$, i.e., we will construct $B_{h}=: \Lambda_{h}^{k} \rightarrow \Lambda_{h}^{k}$ such that the spectral equivalence relation (10.12) holds and such that $B_{h}$ can be evaluated cheaply. Before doing so, we note the correspondence between the operators $I+\mathrm{d}^{*} \mathrm{~d}=I+\mathrm{d}_{k}^{*} \mathrm{~d}_{k}$ and well known differential operators in the three dimensional case, if the $k$-forms are identified with the associated proxy fields.

The operators $I+\mathrm{d}^{*} \mathrm{~d}$

Consider the case $n=3$, and identify $k$-forms by their associated proxy fields as described in $\S 2.3$ above. If we consider the case $k=0$, then 0 forms 
correspond to real-valued functions and the inner product $\langle\omega, \omega\rangle_{H \Lambda}$ to the standard inner product of $H^{1}(\Omega)$ given by

$$
\langle\omega, \omega\rangle+\langle\operatorname{grad} \omega, \operatorname{grad} \omega\rangle .
$$

Furthermore, $I+\mathrm{d}_{0}^{*} \mathrm{~d}_{0}$ corresponds to $I-\operatorname{div} \operatorname{grad}=I-\Delta$. Here $\Delta$ is the standard Laplace operator on scalar functions, and $\langle\cdot, \cdot\rangle$ the inner product in $L^{2}(\Omega)$. If $k=1$ and $\omega \in H \Lambda^{1}(\Omega)$ is identified with a vector field, then $\langle\omega, \omega\rangle_{H \Lambda}$ becomes

$$
\langle\omega, \omega\rangle+\langle\operatorname{curl} \omega, \operatorname{curl} \omega\rangle,
$$

and $I+\mathrm{d}_{1}^{*} \mathrm{~d}_{1}$ is represented by $I+$ curl curl. For $k=2$, the inner product corresponds to

$$
\langle\omega, \omega\rangle+\langle\operatorname{div} \omega, \operatorname{div} \omega\rangle,
$$

and $I+\mathrm{d}_{2}^{*} \mathrm{~d}_{2}$ is represented by $I-\operatorname{grad} \operatorname{div}$. Finally, for $k=3$ the inner product is simply the $L^{2}$-inner product and $I+\mathrm{d}_{3}^{*} \mathrm{~d}_{3}$ reduces to the identity operator.

Note that $I+\mathrm{d}_{0}^{*} \mathrm{~d}_{0}$ corresponds to a standard second-order elliptic differential operator. In fact, the modern preconditioning techniques built on space decompositions, such as domain decomposition and multigrid algorithms, are tailored to operators of this form. On the other hand, the differential operators corresponding to $I+\mathrm{d}_{k}^{*} \mathrm{~d}_{k}$, for $k=1$ and $k=2$, are not standard second-order elliptic operators. For example, the operator $I+$ curl curl behaves like a second-order differential operator on curl fields, but degenerates to the identity on gradient fields. The operator $I-\operatorname{grad} \operatorname{div}$ has similar properties. These properties just mirror the fact that the operator $I+\mathrm{d}_{k}^{*} \mathrm{~d}_{k}$ is the identity operator on $\mathfrak{B}^{k}$, but acts like a second order differential operator on $\mathfrak{Z}^{k \perp}$.

It is well known that the simplest preconditioners constructed for discretizations of standard second-order elliptic operators will not work for the operators $I+\operatorname{curl}$ curl and $I-$ grad div. In fact, it seems that a necessary condition for the construction of optimal preconditioners is the existence of proper Hodge decompositions for the discrete spaces. Early work on this topic in two and three space dimensions can be found in Vassilevski and Wang (1992), Hiptmair (1997), Hiptmair (1999b), Hiptmair and Toselli (2000), Arnold, Falk and Winther (1997b) and Arnold, Falk and Winther (2000). Here we shall indicate how such a construction can be carried out for general $n$ and $k$ using the spaces $\Lambda_{h}^{k}$ introduced above.

The discretization of the operator $I+\mathrm{d}^{*} \mathrm{~d}$

As approximations of the differential operator $I+\mathrm{d}^{*} \mathrm{~d}$, we define the operator $A_{h}: \Lambda_{h}^{k} \rightarrow\left(\Lambda_{h}^{k}\right)^{*}=\Lambda_{h}^{k}$ by

$$
\left\langle A_{h} \omega, \tau\right\rangle=\langle\omega, \tau\rangle_{H \Lambda}, \quad \tau \in \Lambda_{h}^{k}
$$


The operator $A_{h}$ is symmetric and positive definite with respect to the $L^{2}$ inner product $\langle\cdot, \cdot\rangle$. Note that the inequalities (10.12) are equivalent to the statement that the spectrum of the operator $A_{h}^{-1} B_{h}^{-1}$ is contained in the interval $\left[c_{2}^{-1}, c_{1}^{-1}\right]$. This is again equivalent to the property that the spectrum of $B_{h} A_{h}$ is contained in the interval $\left[c_{1}, c_{2}\right]$, or

$$
c_{1}\|\omega\|_{H \Lambda}^{2} \leq\left\langle B_{h} A_{h} \omega, \omega\right\rangle_{H \Lambda} \leq c_{2}\|\omega\|_{H \Lambda}^{2}, \quad \omega \in \Lambda_{h}^{k} .
$$

\section{A two-level preconditioner}

Let $\mathcal{T}_{h}$ be the triangulation of $\Omega$ used to construct the finite element space $\Lambda_{h}^{k}$. Assume further that $\mathcal{T}_{h}$ is obtained from a refinement of a coarser triangulation $\mathcal{T}_{h_{0}}$, where the mesh parameter $h_{0}>h$. If $\Lambda_{h_{0}}^{k}$ is the corresponding subspace of $H \Lambda^{k}(\Omega)$, constructed from the same polynomial functions as $\Lambda_{h}^{k}$, then $\Lambda_{h_{0}}^{k} \subset \Lambda_{h}^{k}$. In fact, we obtain a commuting diagram of the form

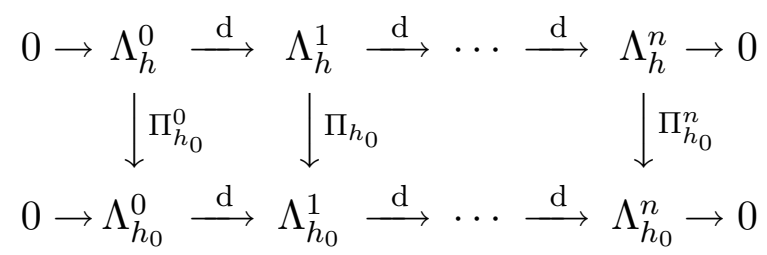

where the projection operators $\Pi_{h_{0}}^{k}$ are the canonical interpolation projections onto the spaces $\Lambda_{h_{0}}^{k}$.

The inverse of the discrete operator $A_{h_{0}}: \Lambda_{h_{0}}^{k} \rightarrow \Lambda_{h_{0}}^{k}$, defined by (10.13) with $\Lambda_{h}^{k}$ replaced by $\Lambda_{h_{0}}^{k}$, will be used to define the two-level preconditioner $B_{h}$ for the operator $A_{h}$. Furthermore, we will assume that the coarse mesh $\mathcal{T}_{h_{0}}$ is not too coarse, in the sense that there is a constant $C>0$, independent of $h$, such that

$$
h_{0} \leq C h \text {. }
$$

The following two-level version of the dual estimate of Theorem 9.2, which is a generalization to $n$ dimensions of Propositions 4.3 and 4.4 of Arnold et al. (2000), will be useful below.

Theorem 10.1. Assume that domain is convex or, more generally, 1regular (see (7.24)), and that (10.14) holds. Suppose further that $\omega \in \Lambda_{h}^{k}$ satisfies

$$
\langle\omega, \tau\rangle_{H \Lambda}=0, \quad \tau \in \Lambda_{h_{0}}^{k}
$$

and has the discrete Hodge decomposition

$$
\omega=\omega_{\mathrm{d}, h}+\omega_{\delta, h}=\mathrm{d} \sigma_{h}+\omega_{\delta, h},
$$

where $\sigma_{h} \in \mathfrak{Z}_{h}^{(k-1) \perp}$, and $\omega_{\delta, h} \in \mathfrak{Z}_{h}^{k \perp}$. Then there is a constant $c$, independent of $h$, such that

$$
\left\|\sigma_{h}\right\| \leq \operatorname{ch}\|\omega\|, \quad \text { and } \quad\left\|\omega_{\delta, h}\right\| \leq \operatorname{ch}\|\omega\|_{H \Lambda} .
$$


Proof. The orthogonality condition implies that

$$
\left\langle\mathrm{d} \sigma_{h}, \mathrm{~d} \mu\right\rangle=0, \quad \mu \in \Lambda_{h_{0}}^{k-1} .
$$

Introduce $u \in \mathfrak{Z}^{(k-1) \perp}$ as the solution of

$$
\langle\mathrm{d} u, \mathrm{~d} v\rangle=\left\langle\sigma_{h}, v\right\rangle, \quad v \in \mathfrak{Z}^{(k-1) \perp} .
$$

Then $\mathrm{d} u \in H \Lambda^{k}(\Omega) \cap \stackrel{\circ}{H}^{*} \Lambda^{k}(\Omega)=H^{1} \Lambda^{k}(\Omega)$, with $\delta \mathrm{d} u=\sigma_{h}$. Therefore,

$$
\begin{aligned}
\left\|\sigma_{h}\right\|^{2} & =\left\langle\mathrm{d} \sigma_{h}, \mathrm{~d} u\right\rangle=\left\langle\mathrm{d} \sigma_{h},\left(I-\tilde{\Pi}_{h_{0}}\right) d u\right\rangle \\
& \leq c h\left\|\mathrm{~d} \sigma_{h}\right\|\|\mathrm{d} u\|_{H^{1} \Lambda} \leq c h\|\omega\|\left\|\sigma_{h}\right\|,
\end{aligned}
$$

which shows the first bound. For the second bound we compare the discrete Hodge decomposition of $\omega$, given above, with the corresponding continuous decomposition

$$
\omega=\mathrm{d} \sigma+\omega_{\delta}, \quad \sigma \in \mathfrak{Z}^{(k-1) \perp}, \omega_{\delta} \in \mathfrak{Z}^{k \perp} .
$$

Then $\omega_{\delta} \in H^{1} \Lambda^{k}(\Omega)$ with $\left\|\omega_{\delta}\right\| \leq c\|\omega\|_{H \Lambda}$. Furthermore, from Theorem 9.2, we obtain

$$
\left\|\omega_{\delta}\right\| \leq C h\|\omega\|_{H \Lambda}
$$

Since $\mathrm{d} \omega_{\delta}=\mathrm{d} \omega_{\delta, h}$, we get by Lemma 5.10 that

$$
\left\|\omega_{\delta, h}-\omega_{\delta}\right\| \leq C h\left\|\omega_{\delta}\right\|_{H^{1} \Lambda} \leq C h\|\omega\|_{H \Lambda} .
$$

In order to simplify the notation, we will simply write $A_{0}$ instead of $A_{h_{0}}$, and $\Lambda_{0}^{k}$ instead of $\Lambda_{h_{0}}^{k}$. Furthermore, we let $P_{0}=P_{h_{0}}: H \Lambda^{k}(\Omega) \rightarrow \Lambda_{0}^{k}$ and $Q_{0}=Q_{h_{0}}: L^{2} \Lambda^{k}(\Omega) \rightarrow \Lambda_{0}^{k}$ be the orthogonal projections with respect to the inner products $\langle\cdot, \cdot\rangle_{H \Lambda}$ and $\langle\cdot, \cdot\rangle$, respectively. With this notation it is straightforward to verify that

$$
A_{0} P_{0}=Q_{0} A_{h}
$$

In order to construct the preconditioner $B_{h}$, we will need a smoothing operator $R_{h}: \Lambda_{h}^{k} \rightarrow \Lambda_{h}^{k}$. (Note that in the context of preconditioners, a smoothing operator refers to an approximate inverse, typically computed by a classical iteration, and is not related to mollification procedure discussed in Section 5.4.) Typically, the operator $R_{h}$ will approximate $A_{h}^{-1}$, but with high accuracy only on a part of the space. More precisely, the operator $R_{h}$ is assumed to be symmetric and positive definite with respect to $\langle\cdot, \cdot\rangle$. In addition, we assume that there is a positive constant $c_{0}$, independent of $h$, such that

$$
\left\langle R_{h}^{-1} \omega, \omega\right\rangle \leq c_{0}\|\omega\|_{H \Lambda}^{2}, \quad \omega \in\left(I-P_{0}\right) \Lambda_{h}^{k}
$$

and that $R_{h}$ satisfies

$$
\left\langle R_{h} A_{h} \omega, \omega\right\rangle_{H \Lambda} \leq\|\omega\|_{H \Lambda}^{2}, \quad \omega \in \Lambda_{h}^{k} .
$$


The first condition states that the smoothing operator $R_{h}$ approximates $A_{h}$ well for highly oscillating functions (orthogonal to the coarse space $\Lambda_{0}^{k}$ ), while the second condition states that $R_{h}$ is properly scaled.

The two-level preconditioner $B_{h}: \Lambda_{h}^{k} \rightarrow \Lambda_{h}^{k}$ is defined as $\omega \mapsto B_{h} \omega=\sigma_{3}$ where $\sigma_{1}, \sigma_{2}, \sigma_{3}$ is defined by the following algorithm:

(i) $\sigma_{1}=R_{h} \omega$,

(ii) $\sigma_{2}=\sigma_{1}-A_{0}^{-1} Q_{0}\left(A_{h} \sigma_{1}-\omega\right)$,

(iii) $\sigma_{3}=\sigma_{2}-R_{h}\left(A_{h} \sigma_{2}-\omega\right)$.

In steps (i) and (ii), the smoothing operator $R_{h}$ is evaluated, while in step (iii) awe must find $\mu_{0} \in \Lambda_{0}^{k}$ as the solution of the discrete problem

$$
\left\langle\mu_{0}, \tau\right\rangle_{H \Lambda}=\left\langle\sigma_{1}, \tau\right\rangle_{H \Lambda}-\langle\omega, \tau\rangle \quad \tau \in \Lambda_{0}^{k}
$$

Therefore, in order to evaluate the preconditioner $B_{h}$, approximating $A_{h}^{-1}$, $R_{h}$ is evaluated twice, and $A_{h}$ and $A_{0}^{-1}$ once.

Such a two-level algorithm as presented here may not be very efficient, since it is usually necessary to assume that condition (10.14) holds. As a consequence, the linear system associated to $A_{0}$ is not much smaller than the system associated to $A_{h}$. Therefore, in most practical computations a multilevel algorithm should be used. Such an algorithm is defined by using the algorithm above recursively, where the operator $A_{0}^{-1}$ is replaced by the use of the two-level algorithm at the coarser level, and repeated until a sufficiently small system is obtained. However, for the theoretical discussion here, where the main purpose is to discuss how the discrete Hodge decomposition for the spaces $\Lambda_{h}^{k}$ enters into the construction of the smoothing operators $R_{h}$, the two-level algorithm is sufficient. We will therefore restrict the discussion here to the two-level algorithm above.

Properties of the preconditioner $B_{h}$

In the rest of this section, only the discrete spaces will occur. For simplicity of notation, we will therefore drop the subscript $h$, and just write $\Lambda^{k}, A, B$, and $R$ instead of $\Lambda_{h}^{k}, A_{h}, B_{h}$, and $R_{h}$. It is a simple computation to show that the two-level preconditioner $B$ satisfies the identity

$$
I-B A=(I-R A)\left(I-P_{0}\right)(I-R A) .
$$

Furthermore, all the eigenvalues of $B A$ are contained in the interval $\left[1 / c_{0}, 1\right]$, where $c_{0}$ is the positive constant appearing in (10.15). This is a consequence of the theorem below, which is a two-level version of a pioneering result first proved in Braess and Hackbusch (1983).

Theorem 10.2. If the assumptions (10.15) and (10.16) hold, then the two-level preconditioner $B: \Lambda^{k} \mapsto \Lambda^{k}$ defined above satisfies

$$
0 \leq\langle(I-B A) \omega, \omega\rangle_{H \Lambda} \leq\left(1-\frac{1}{c_{0}}\right)\|\omega\|_{H \Lambda}, \quad \omega \in \Lambda^{k} .
$$


Proof. The left inequality follows since (10.17) implies that

$$
\langle(I-B A) \omega, \omega\rangle_{H \Lambda}=\left\|\left(I-P_{0}\right)(I-R A) \omega\right\|_{H \Lambda}^{2} \geq 0 .
$$

Let $\tau \in\left(I-P_{0}\right) \Lambda^{k}$. To obtain the right inequality, we note that it follows from (10.15) and the Cauchy-Schwarz inequality that

$$
\langle R A \tau, \tau\rangle_{H \Lambda} \geq c_{0}^{-1}\|\tau\|_{H \Lambda}^{2} .
$$

This follows since

$$
\begin{aligned}
\|\tau\|_{H \Lambda}^{2} & =\left\langle R^{-1 / 2} \tau, R^{1 / 2} A \tau\right\rangle \\
& \leq\left\langle R^{-1} \tau, \tau\right\rangle^{1 / 2}\langle R A \tau, \tau\rangle_{H \Lambda}^{1 / 2} \\
& \leq c_{0}\|\tau\|_{H \Lambda}\langle R A \tau, \tau\rangle_{H \Lambda}^{1 / 2} .
\end{aligned}
$$

Note that the operator $I-R A$ is symmetric with respect to the inner product $\langle\cdot, \cdot\rangle_{H \Lambda}$, and as a consequence of (10.16), positive semidefinite. Therefore, we obtain from (10.18) that

$$
\begin{aligned}
\|(I-R A) \tau\|_{H \Lambda}^{2} & =\|\tau\|_{H \Lambda}^{2}-\langle(2 I-R A) R A \tau, \tau\rangle_{H \Lambda} \\
& \leq\|\tau\|_{H \Lambda}^{2}-\langle R A \tau, \tau\rangle_{H \Lambda} \\
& \leq\left(1-\frac{1}{c_{0}}\right)\|\tau\|_{H \Lambda}^{2} .
\end{aligned}
$$

This final inequality further implies that

$$
\left\|(I-R A)\left(I-P_{0}\right) \omega\right\|_{H \Lambda} \leq\left(1-\frac{1}{c_{0}}\right)\|\omega\|_{H \Lambda}, \quad \omega \in \Lambda^{k} .
$$

In other words, the operator norm $\left\|(I-R A)\left(I-P_{0}\right)\right\|_{\mathcal{L}\left(H \Lambda^{k}(\Omega), H \Lambda^{k}(\Omega)\right)}$ is bounded by $1-1 / c_{0}$. Hence, the corresponding norm of the adjoint operator, $\left(I-P_{0}\right)(I-R A)$ admits the same bound, and this is equivalent to the lower bound.

\section{Schwarz smoothers}

The main challenge in obtaining effective preconditioners for the discrete operator $A: \Lambda^{k} \mapsto \Lambda^{k}$ is the construction of the smoothing operator $R$. In fact, it is only in this part of the construction that the special properties of the operator $A$ and the discrete spaces $\Lambda^{k}$ will enter. We will consider so called Schwarz smoothers. These smoothers will be defined from a collection of subspaces $\left\{\Lambda_{j}^{k}\right\}_{j=1}^{m}$ of $\Lambda^{k}$. We will assume that

$$
\Lambda^{k}=\sum_{j=1}^{m} \Lambda_{j}^{k}
$$

in the sense that all elements $\omega \in \Lambda^{k}$ can be written as $\omega=\sum_{j} \omega_{j}$, where $\omega_{j} \in \Lambda_{j}^{k}$. The decomposition is not required to be unique. The spaces $\Lambda_{j}^{k}$ 
should be of low dimension, independent of the triangulation $\mathcal{T}_{h}$. In fact, frequently they are taken to be generated by a single basis function.

From the family of subspaces $\left\{\Lambda_{j}^{k}\right\}$, we can can construct two different smoothing operators, usually referred to as the multiplicative and the additive Schwarz smoother. The multiplicative Schwarz smoother is given by the following algorithm.

Define $R \omega=\sigma_{2 m}$, where $\sigma_{0}=0$,

$$
\begin{aligned}
\sigma_{j}=\sigma_{j-1}-P_{j}\left(\sigma_{j-1}-A^{-1} \omega\right), & j & =1,2, \ldots, m, \\
\sigma_{j}=\sigma_{j-1}-P_{2 m+1-j}\left(\sigma_{j-1}-A^{-1} \omega\right), & j & =m+1, m+2, \ldots, 2 m .
\end{aligned}
$$

Here $P_{j}$ is the $H \Lambda$-orthogonal projection onto $\Lambda_{j}^{k}$. Note that if $A_{j}$ is the representation of $A$ on $\Lambda_{j}^{k}$, defined from (10.13) with $\Lambda^{k}$ replaced by $\Lambda_{j}^{k}$, and $Q_{j}$ is the $L^{2}$-orthogonal projection onto $\Lambda_{j}^{k}$, then $P_{j} A^{-1}=A_{j}^{-1} Q_{j}$. This shows that that in each step of the algorithm above, only 'the local operator' $A_{j}$ has to be inverted.

The corresponding additive smoother is given as a suitable scaling of the operator $R_{a}=\sum_{j=1}^{m} P_{j} A^{-1}$. For simplicity, we will restrict the discussion here to the multiplicative Schwarz smoother. This smoothing operator will always satisfy the condition (10.16). In fact, it is straightforward to verify that

$$
\langle(I-B A) \omega, \omega\rangle_{H \Lambda}=\left\langle\left(I-P_{m}\right) \ldots\left(I-P_{1}\right) \omega,\left(I-P_{m}\right) \ldots\left(I-P_{1}\right) \omega\right\rangle_{H \Lambda} \geq 0 .
$$

Furthermore, the first condition can be verified by applying the following result.

Theorem 10.3. Suppose that the subspaces $\left\{\Lambda_{j}^{k}\right\}$ of $\Lambda^{k}$ satisfy the two conditions

$$
\sum_{i=1}^{m} \sum_{j=1}^{m} \mid\left\langle\omega_{i}, \tau_{j}\right\rangle_{H \Lambda} \leq a_{1}\left(\sum_{i=1}^{m}\left\|\omega_{i}\right\|_{H \Lambda}^{2}\right)^{1 / 2}\left(\sum_{j=1}^{m}\left\|\tau_{j}\right\|_{H \Lambda}^{2}\right)^{1 / 2},
$$

where $\omega_{i} \in \Lambda_{i}^{k}$ and $\tau_{j} \in \Lambda_{j}^{k}$, and

$$
\inf _{\substack{\omega_{i} \in \Lambda_{i}^{k} \\ \omega=\sum \omega_{i}}} \sum_{i=1}^{m}\left\|\omega_{i}\right\|_{H \Lambda}^{2} \leq a_{2}\|\omega\|_{H \Lambda}^{2}, \quad \omega \in\left(I-P_{0}\right) \Lambda^{k}
$$

for some positive constants $a_{1}$ and $a_{2}$. Then the corresponding multiplicative Schwarz smoother satisfies condition (10.15) with $c_{0}=a_{1}^{2} a_{2}$.

The second condition here is frequently referred to as a stable decomposition property. This condition is in fact closely related to the additive 
Schwarz operator $R_{a}$ by the relation

$$
\left\langle R_{a}^{-1} \omega, \omega\right\rangle=\inf _{\substack{\omega_{i} \in \Lambda_{i}^{k} \\ \omega=\sum \omega_{i}}} \sum_{i=1}^{m}\left\|\omega_{i}\right\|_{H \Lambda}^{2} .
$$

Furthermore, the first condition can be used to obtain the bound

$$
\left\langle R_{a} \omega, \omega\right\rangle \leq a_{1}^{2}\langle R \omega, \omega\rangle .
$$

In fact, results of this type can be found in many places, i.e., in Bramble (1993), Dryja and Widlund (1995), Smith, Bjørstad and Gropp (1996), Xu (1992): see also Section 3 of Arnold et al. (2000). We will therefore not give a proof here.

The choice of space decomposition

Let $\left\{\Omega_{j}\right\}$ be the subsets of $\Omega$ defined by

$$
\Omega_{j}=\left\{x \in \Omega \mid \operatorname{supp} \omega \subset \bar{\Omega}_{j}, \omega \in \Lambda_{j}^{k}\right\} .
$$

Note that if $\Lambda_{j}^{k}$ is generated by a single basis function, then any point of $\Omega$ will only be contained in a finite number of the subdomains $\Omega_{j}$, and the number of overlapping subdomains will not grow with $h$. The same property will hold if the spaces $\Lambda_{j}^{k}$ are generated by a fixed number of neighbouring basis functions. Furthermore, the constant $a_{1}$ appearing in (10.19) can be bounded by the sum of the characteristic functions of the domains $\Omega_{j}$. So for a locally defined Schwarz smoother, the constant $a_{1}$ will be a priori bounded independently of $h$.

The stable decomposition property, (10.20), is harder to fulfil. For the rest of this section, let $\Gamma_{i}^{k}$ be the one-dimensional subspaces of $\Lambda^{k}$ generated by each basis function. Then, for any $\omega \in \Lambda^{k}$ the decomposition

$$
\omega=\sum_{i} \omega_{i}, \quad \omega_{i} \in \Gamma_{i}^{k}
$$

is unique. Furthermore, by using equivalence of norms on the reference element, we have

$$
\sum_{i}\left\|\omega_{i}\right\|^{2} \leq C_{1}\|\omega\|^{2}, \quad \text { and } \quad\left\|\mathrm{d} \omega_{i}\right\| \leq C_{1} h^{-1}\left\|\omega_{i}\right\|,
$$

for each $\omega \in \Lambda^{k}$, where the constant $C_{1}$ is independent of $h$.

Consider first the case $k=0$ and assume that the hypotheses of Theorem 10.1 hold. It follows from the standard duality argument of finite element theory that for all $\omega \in\left(I-P_{0}\right) \Lambda^{0}$, which are orthogonal to constants, we have the estimate

$$
\|\omega\| \leq c h\|\mathrm{~d} \omega\| .
$$


In fact, this result is also contained in Theorem 10.1. The property (10.22) reflects the fact that the elements of $\left(I-P_{0}\right) \Lambda^{0}$ are highly oscillating functions. However, estimate (10.21) gives

$$
\sum_{i=1}^{m}\left\|\omega_{i}\right\|_{H \Lambda}^{2} \leq C_{1} h^{-2}\|\omega\|^{2}
$$

and therefore (10.22) implies that (10.20) holds.

Consider next the case when $1 \leq k<n$. Of course, in this case we can not expect the bound (10.22) to hold in general, since the $L^{2}$-norm and the $H \Lambda$-norm coincide on $\mathfrak{Z}^{k}$. Instead we have to rely on the estimates given in Theorem 10.1. Hence, it seems necessary that the discrete Hodge decomposition will enter the construction.

The smoothers introduced by Vassilevski and Wang (1992) in two space dimensions, and Hiptmair (1997) in three dimensions use the decomposition

$$
\sum_{i} \mathrm{~d} \Gamma_{i}^{k-1}+\sum_{j} \Gamma_{j}^{k}
$$

of $\Lambda^{k}$. This decomposition is stable. In order to see this let $\omega \in\left(I-P_{0}\right) \Lambda^{k}$ be decomposed as

$$
\omega=\mathrm{d} \sigma+\omega_{\delta}=\sum_{i} \mathrm{~d} \sigma_{i}+\sum_{j} \omega_{\delta, j}
$$

where $\sigma$ and $\omega_{\delta}$ are as in Theorem 10.1, $\sigma_{i} \in \Gamma_{i}^{k-1}$ and $\omega_{\delta, j} \in \Gamma_{j}^{k}$. Using the estimates from (10.21) and Theorem 10.1 we now have

$$
\begin{aligned}
\sum_{i}\left\|\mathrm{~d} \sigma_{i}\right\|^{2}+\sum_{j}\left\|\omega_{\delta, j}\right\|^{2} & \leq c h^{-2}\left(\|\sigma\|^{2}+\left\|\omega_{\delta}\right\|^{2}\right) \\
& \leq c\|\omega\|_{H \Lambda}^{2}
\end{aligned}
$$

which shows (10.20).

As an alternative to the decomposition (10.23), which employs the basis functions of both the spaces $\Lambda^{k-1}$ and $\Lambda^{k-1}$, the construction carried out in Arnold et al. (2000) only utilizes the basis functions of the space $\Lambda^{k}$. However, the subspaces will then not be one-dimensional, since one has to make sure that all the spaces $\mathrm{d} \Gamma_{i}^{k-1}$ are contained in at least one subspace. This will typically lead to the property that a low $(<10)$-dimensional linear system has to be solved for each step of the Schwarz algorithm. We refer to Arnold et al. (2000) for further details. 


\section{The elasticity equations}

\subsection{Introduction}

The equations of linear elasticity can be written as a system of equations of the form

$$
A \sigma=\epsilon u, \quad \operatorname{div} \sigma=f \quad \text { in } \Omega .
$$

Here the unknowns $\sigma$ and $u$ denote the stress and displacement fields engendered by a body force $f$ acting on a linearly elastic body which occupies a region $\Omega \subset \mathbb{R}^{n}$, with boundary $\Gamma$. Then $\sigma$ takes values in the space $\mathbb{S}=\mathbb{R}_{\mathrm{sym}}^{n \times n}$ of symmetric second-order tensors and $u$ takes values in $\mathbb{V}=\mathbb{R}^{n}$. The differential operator $\epsilon$ is the symmetric part of the gradient, div denotes the divergence operator taking tensor fields to vector fields, and the fourth order compliance tensor $A=A(x): \mathbb{S} \rightarrow \mathbb{S}$ is a bounded and symmetric, uniformly positive definite operator reflecting the properties of the material at each point. If the body is clamped on the boundary $\partial \Omega$ of $\Omega$, then the proper boundary condition for the system (11.1) is $u=0$ on $\partial \Omega$. For simplicity, this boundary condition will be assumed throughout most of the discussion here. However, there are issues that arise when other boundary conditions are assumed (e.g., traction boundary conditions $\sigma n=0$ ). The modifications needed to deal with such boundary conditions are discussed in the final subsection of this part of the paper.

The system (11.1) can be formulated weakly in a number of ways. One is to seek $\sigma \in H(\operatorname{div}, \Omega ; \mathbb{S})$, the space of square-integrable symmetric tensor fields with square-integrable divergence, and $u \in L^{2}(\Omega ; \mathbb{V})$, satisfying

$$
\begin{array}{ccc}
\int_{\Omega}(A \sigma: \tau+\operatorname{div} \tau \cdot u) \mathrm{d} x & =0, & \tau \in H(\operatorname{div}, \Omega ; \mathbb{S}), \\
\int_{\Omega} \operatorname{div} \sigma \cdot v \mathrm{~d} x=\int_{\Omega} f v \mathrm{~d} x, & v \in L^{2}(\Omega ; \mathbb{V}) .
\end{array}
$$

A second weak formulation, that also enforces the symmetry weakly, seeks $\sigma \in H(\operatorname{div}, \Omega ; \mathbb{M}), u \in L^{2}(\Omega ; \mathbb{V})$, and $p \in L^{2}(\Omega ; \mathbb{K})$ satisfying

$$
\begin{array}{rlrl}
\int_{\Omega}(A \sigma: \tau+\operatorname{div} \tau \cdot u+\tau: p) \mathrm{d} x & =0, & & \tau \in H(\operatorname{div}, \Omega ; \mathbb{M}), \\
\int_{\Omega} \operatorname{div} \sigma \cdot v \mathrm{~d} x=\int_{\Omega} f v \mathrm{~d} x, & v \in L^{2}(\Omega ; \mathbb{V}), \\
\int_{\Omega} \sigma: q \mathrm{~d} x=0, & & q \in L^{2}(\Omega ; \mathbb{K}),
\end{array}
$$

where $\mathbb{M}$ is the space of second-order tensors, $\mathbb{K}$ the subspace of skewsymmetric tensors, and the compliance tensor $A(x)$ is now considered as a symmetric and positive definite operator mapping $\mathbb{M}$ into $\mathbb{M}$. In the 
isotropic case, the mapping $\sigma \mapsto A \sigma$ has the form

$$
A \sigma=\frac{1}{2 \mu}\left(\sigma-\frac{\lambda}{2 \mu+n \lambda} \operatorname{tr}(\sigma) I\right),
$$

where $\lambda(x), \mu(x)$ are positive scalar coefficients, the Lamé coefficients.

Stable finite element discretizations with reasonable computational complexity based on the variational formulation (11.2) have proved very difficult to construct. In two space dimensions, the first stable finite elements with polynomial shape functions were presented in Arnold and Winther (2002). For the lowest order element, the approximate stress space is composed of certain piecewise cubic functions, while the displacement space consists of piecewise linear functions. In three dimensions, a piecewise quartic stress space is constructed with 162 degrees of freedom on each tetrahedron (Adams and Cockburn 2005). Another approach which has been discussed in the two-dimensional case is the use of composite elements, in which the approximate displacement space consists of piecewise polynomials with respect to one triangulation of the domain, while the approximate stress space consists of piecewise polynomials with respect to a different, more refined, triangulation (Fraeijs de Veubeke 1965, Watwood and Hartz 1968, Johnson and Mercier 1978, Arnold, Douglas and Gupta 1984b).

Because of the lack of suitable mixed elasticity elements that strongly impose the symmetry of the stresses, a number of authors have developed approximation schemes based on the weak symmetry formulation (11.3): see Fraeijs de Veubeke (1965), Amara and Thomas (1979), Arnold, Brezzi and Douglas (1984a), Stenberg (1986), Stenberg (1988a), Stenberg (1988b), Arnold and Falk (1988), Morley (1989), Stein and Rolfes (1990), Farhloul and Fortin (1997). Although (11.2) and (11.3) are equivalent on the continuous level, an approximation scheme based on (11.3) may not produce a symmetric approximation to the stress tensor, depending on the choices of finite element spaces.

In this section of the paper, we build on the techniques derived in the previous sections to develop and analyse finite element approximations of the equations of linear elasticity based on the mixed formulation (11.3) with weak symmetry. The basic ideas first appeared in Arnold, Falk and Winther (2006c) in two dimensions and Arnold, Falk and Winther (2005) in three dimensions.

In order to write (11.3) in the language of exterior calculus we will use the spaces of vector-valued differential forms presented in Section 6. The domain $\Omega$ is a bounded open set in $\mathbb{R}^{n}, \mathbb{V}=\mathbb{R}^{n}$ denotes its tangent space at any point, and $\mathbb{K}=\mathbb{V} \wedge \mathbb{V}$ the space of bivectors defined in Section 2.1, which is identified with the space of skew-symmetric linear operators $\mathbb{V} \rightarrow \mathbb{V}$. As explained in Section 6 , it is natural to seek the stress $\sigma$ in the space $\Lambda^{n-1}(\Omega ; \mathbb{V})$, so that if $\Gamma$ is an $(n-1)$-dimensional surface embedded in $\bar{\Omega}, e . g$. , a portion of 
the boundary of a subdomain of $\Omega$, then $\int_{\Gamma} \sigma$ is a vector representing force. The Hodge star operator then represents the stress by $\star \sigma \in \Lambda^{1}(\Omega ; \mathbb{V})$, which means it defines a linear operator $\mathbb{V} \rightarrow \mathbb{V}$ (i.e., a second-order tensor or matrix) at every point: this is the classical representation of stress. In Proposition 6.1 , we showed that the operator $S=S_{n-1}: \Lambda^{n-1}(\Omega ; \mathbb{V}) \rightarrow \Lambda^{n}(\Omega ; \mathbb{K})$ defined in (6.2) corresponds (up to a factor of \pm 2 ) to taking the skewsymmetric part of its argument. Thus the elasticity problem (11.3) becomes: find $(\sigma, u, p) \in H \Lambda^{n-1}(\Omega ; \mathbb{V}) \times L^{2} \Lambda^{n}(\Omega ; \mathbb{V}) \times L^{2} \Lambda^{n}(\Omega ; \mathbb{K})$ such that

$$
\begin{aligned}
\langle A \sigma, \tau\rangle+\langle\mathrm{d} \tau, u\rangle-\langle S \tau, p\rangle & =0, & & \tau \in H \Lambda^{n-1}(\Omega ; \mathbb{V}), \\
\langle\mathrm{d} \sigma, v\rangle & =\langle f, v\rangle, & & v \in L^{2} \Lambda^{n}(\Omega ; \mathbb{V}), \\
\langle S \sigma, q\rangle & =0, & & q \in L^{2} \Lambda^{n}(\Omega ; \mathbb{K}) .
\end{aligned}
$$

This problem is well-posed in the sense that, for each $f \in L^{2} \Lambda^{n}(\Omega ; \mathbb{V})$, there exists a unique solution $(\sigma, u, p) \in H \Lambda^{n-1}(\Omega ; \mathbb{V}) \times L^{2} \Lambda^{n}(\Omega ; \mathbb{V}) \times L^{2} \Lambda^{n}(\Omega ; \mathbb{K})$, and the solution operator is a bounded operator

$$
L^{2} \Lambda^{n}(\Omega ; \mathbb{V}) \rightarrow H \Lambda^{n-1}(\Omega ; \mathbb{V}) \times L^{2} \Lambda^{n}(\Omega ; \mathbb{V}) \times L^{2} \Lambda^{n}(\Omega ; \mathbb{K})
$$

This will follow from the general theory of such saddle point problems (Brezzi 1974) once we establish two conditions:

(W1) $\|\tau\|_{H \Lambda}^{2} \leq c_{1}\langle A \tau, \tau\rangle$ whenever $\tau \in H \Lambda^{n-1}(\Omega ; \mathbb{V})$ satisfies $\langle\mathrm{d} \tau, v\rangle=0$ $\forall v \in L^{2} \Lambda^{n}(\Omega ; \mathbb{V})$ and $\langle S \tau, q\rangle=0 \forall q \in L^{2} \Lambda^{n}(\Omega ; \mathbb{K})$,

(W2) for all nonzero $(v, q) \in L^{2} \Lambda^{n}(\Omega ; \mathbb{V}) \times L^{2} \Lambda^{n}(\Omega ; \mathbb{K})$, there exists nonzero $\tau \in H \Lambda^{n-1}(\Omega ; \mathbb{V})$ with $\langle\mathrm{d} \tau, v\rangle-\langle S \tau, q\rangle \geq c_{2}\|\tau\|_{H \Lambda}(\|v\|+\|q\|)$,

for some positive constants $c_{1}$ and $c_{2}$. The first condition is obvious (and does not even utilize the orthogonality of $S \tau$ ). However, the second condition is more subtle. We will verify it in Theorem 11.1 below.

We next consider a finite element discretizations of (11.4). For this, we choose families of finite-dimensional subspaces $\Lambda_{h}^{n-1}(\mathbb{V}) \subset H \Lambda^{n-1}(\Omega ; \mathbb{V})$, $\Lambda_{h}^{n}(\mathbb{V}) \subset L^{2} \Lambda^{n}(\Omega ; \mathbb{V})$, and $\Lambda_{h}^{n}(\mathbb{K}) \subset L^{2} \Lambda^{n}(\Omega ; \mathbb{K})$, indexed by $h$, and seek the discrete solution $\left(\sigma_{h}, u_{h}, p_{h}\right) \in \Lambda_{h}^{n-1}(\mathbb{V}) \times \Lambda_{h}^{n}(\mathbb{V}) \times \Lambda_{h}^{n}(\mathbb{K})$ such that

$$
\begin{aligned}
\left\langle A \sigma_{h}, \tau\right\rangle+\left\langle\mathrm{d} \tau, u_{h}\right\rangle-\left\langle S \tau, p_{h}\right\rangle & =0, & & \tau \in \Lambda_{h}^{n-1}(\mathbb{V}), \\
\left\langle\mathrm{d} \sigma_{h}, v\right\rangle & =\langle f, v\rangle & & v \in \Lambda_{h}^{n}(\mathbb{V}), \\
\left\langle S \sigma_{h}, q\right\rangle & =0, & & q \in \Lambda_{h}^{n}(\mathbb{K}) .
\end{aligned}
$$

In analogy with the well-posedness of the problem (11.4), the stability of the saddle point system (11.5) will be ensured by the Brezzi stability conditions:

(S1) $\|\tau\|_{H \Lambda}^{2} \leq c_{1}(A \tau, \tau)$ whenever $\tau \in \Lambda_{h}^{n-1}(\mathbb{V})$ satisfies $\langle\mathrm{d} \tau, v\rangle=0$ $\forall v \in \Lambda_{h}^{n}(\mathbb{V})$ and $\langle S \tau, q\rangle=0 \forall q \in \Lambda_{h}^{n}(\mathbb{K})$, 
(S2) for all nonzero $(v, q) \in \Lambda_{h}^{n}(\mathbb{V}) \times \Lambda_{h}^{n}(\mathbb{K})$, there exists nonzero $\tau \in \Lambda_{h}^{n-1}(\mathbb{V})$ with $\langle\mathrm{d} \tau, v\rangle-\langle S \tau, q\rangle \geq c_{2}\|\tau\|_{H \Lambda}(\|v\|+\|q\|)$,

where now the constants $c_{1}$ and $c_{2}$ must be independent of $h$. The difficulty is, of course, to design finite element spaces satisfying these conditions.

Just as there is a close relation between the construction of stable mixed finite element methods for the approximation of the Hodge Laplacian and discrete versions of the de Rham complex, there is also a close relation between mixed finite elements for linear elasticity and discretization of an associated complex, the elasticity complex, which will be derived in the next subsection. The importance of this complex for the stability of discretizations of elasticity was first recognized in Arnold and Winther (2002), where mixed methods for elasticity in two space dimensions were discussed. It turns out that there is also a close, but nonobvious, connection between the elasticity complex and the de Rham complex. This connection is described in Eastwood (2000) and is related to a general construction given in Bernštern, Gel'fand and Gel'fand (1975), called the BGG resolution (see also Čap, Slovák and Souček (2001)). In Arnold et al. (2006c) (two dimensions) and Arnold et al. (2005) (three dimensions), we developed a discrete version of the BGG construction, which allowed us to derive stable mixed finite elements for elasticity in a systematic manner based on the finite element subcomplexes of the de Rham complex described earlier. The resulting elements in both two and three space dimensions are simpler than any derived previously. For example, as we shall see, the simple choice of $\mathcal{P}_{1} \Lambda^{n-1}\left(\mathcal{T}_{h} ; \mathbb{V}\right)$ for stress, $\mathcal{P}_{0} \Lambda^{n}\left(\mathcal{T}_{h} ; \mathbb{V}\right)$ for displacement, and $\mathcal{P}_{0} \Lambda^{n}\left(\mathcal{T}_{h} ; \mathbb{K}\right)$ for the multiplier results in a stable discretization of the problem (11.5). In Figure 11.1, this element is depicted in two dimensions with a conventional finite element diagram that portrays the degrees of freedom on a triangle: for stress the first two moments of its trace on the edges, and for the displacement and multiplier, their integrals on the triangle (two components for displacement, one for the multiplier). Moreover, this element is the lowest order of a family of stable elements in $n$ dimensions utilizing $\mathcal{P}_{r} \Lambda^{n-1}\left(\mathcal{T}_{h} ; \mathbb{V}\right)$ for stress, $\mathcal{P}_{r-1} \Lambda^{n}\left(\mathcal{T}_{h} ; \mathbb{V}\right)$ for displacement, and $\mathcal{P}_{r-1} \Lambda^{n}\left(\mathcal{T}_{h} ; \mathbb{K}\right)$ for the multiplier.
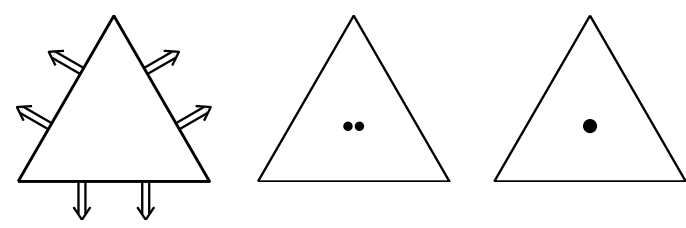

Figure 11.1. Approximation of stress, displacement, and multiplier for the simplest element in two dimensions.

In this section we shall basically follow the approach of Arnold et al. (2006c) and Arnold et al. (2005), but with some simplifications, and in a 
manner which works in $n$ dimensions. In the Section 11.2, we show how an elasticity complex with weakly imposed symmetry can be derived from the de Rham complex. For the convenience of readers more familiar with the classical notation for elasticity, in Section 11.3 we specialize to the cases $n=2$ and $n=3$ and translate our results from the language of exterior calculus to the classical notation. In Section 11.4, we give a proof of the well-posedness of the mixed formulation of elasticity with weak symmetry for the continuous problem, as a guide for establishing a similar result for the discrete problem. In Section 11.5, we derive discrete analogues of the elasticity complex obtained in Section 11.2, beginning from discrete analogues of the de Rham complex, and identify the required properties of the discrete spaces necessary for this construction. These results are then used to establish the main stability result for weakly symmetric mixed finite element approximations of the equations of elasticity in Section 11.6. In Section 11.7 and Section 11.8, we show how our results can be extended to the equations of elasticity with traction boundary conditions and also to obtain some simplified elements.

\subsection{From the de Rham complex to the elasticity complex}

In this subsection we derive the elasticity complex in $n$ dimensions starting with the de Rham complex. Our derivation is strongly influenced by the derivation given in Eastwood (2000) in three dimensions, although we have rearranged it substantially and, moreover, obtain a different complex which is the appropriate one when the symmetry of the stress is imposed weakly. The derivation is such that in Section 11.5 we are able to mimic it on the discrete level and so obtain finite element subcomplexes of the elasticity complex from corresponding finite element subcomplexes of the de Rham complex.

We start with the Cartesian product of two vector-valued de Rham complexes, as in (6.1), one with values in $\mathbb{V}$ and one with values in $\mathbb{K}$. Letting $\mathbb{W}:=\mathbb{K} \times \mathbb{V}$ and writing $\Lambda^{k}(\mathbb{W})$ for the more cumbersome $\Lambda^{k}(\Omega ; \mathbb{K}) \times$ $\Lambda^{k}(\Omega ; \mathbb{V})$, we have the starting complex:

$$
0 \rightarrow \Lambda^{0}(\mathbb{W}) \stackrel{\left(\begin{array}{ll}
\mathrm{d} & 0 \\
0 & \mathrm{~d}
\end{array}\right)}{\longrightarrow} \Lambda^{1}(\mathbb{W}) \stackrel{\left(\begin{array}{ll}
\mathrm{d} & 0 \\
0 & \mathrm{~d}
\end{array}\right)}{\longrightarrow} \cdots \stackrel{\left(\begin{array}{ll}
\mathrm{d} & 0 \\
0 & \mathrm{~d}
\end{array}\right)}{\longrightarrow} \Lambda^{n}(\mathbb{W}) \rightarrow 0
$$

Here the two d operators in the diagonal matrices represent $\mathrm{d} \otimes \mathrm{id}_{\mathbb{K}}$ and $\mathrm{d} \otimes \mathrm{id}_{\mathbb{V}}$, respectively. The elasticity complex will be realized as a subcomplex of an isomorphic image of this complex.

Define $K=K_{k}: \Lambda^{k}(\Omega ; \mathbb{V}) \rightarrow \Lambda^{k}(\Omega ; \mathbb{K})$ by

$$
(K \omega)_{x}\left(v_{1}, \ldots, v_{k}\right)=X(x) \wedge \omega_{x}\left(v_{1}, \ldots, v_{k}\right) .
$$

where $X(x) \in \mathbb{V}$ is the vector corresponding to $x$ as in Section 3.2. Set $S=d K-K \mathrm{~d}$, or, more precisely, $S_{k}=\mathrm{d}_{k+1} K_{k}-K_{k+1} \mathrm{~d}_{k}: \Lambda^{k}(\Omega ; \mathbb{V}) \rightarrow$ 
$\Lambda^{k+1}(\Omega ; \mathbb{K})$. Since $\mathrm{d}^{2}=0$, it follows that

$$
\mathrm{d} S+S \mathrm{~d}=0
$$

Next, we define an isomorphism $\Phi=\Phi_{k}: \Lambda^{k}(\mathbb{W}) \rightarrow \Lambda^{k}(\mathbb{W})$ by

$$
\Phi(\omega, \mu)=(\omega+K \mu, \mu)
$$

with inverse given by

$$
\Phi^{-1}(\omega, \mu)=(\omega-K \mu, \mu)
$$

and an operator $\mathcal{A}=\mathcal{A}_{k}: \Lambda^{k}(\mathbb{W}) \rightarrow \Lambda^{k+1}(\mathbb{W})$ by $\mathcal{A}=\Phi \mathrm{d} \Phi^{-1}$. The operator $\mathcal{A}$ has a simple form. Using the definition of $\Phi$, we obtain for $(\omega, \mu) \in \Lambda^{k}(\mathbb{W})$

$$
\mathcal{A}(\omega, \mu)=\Phi \circ \mathrm{d}(\omega-K \mu, \mu)=\Phi(\mathrm{d} \omega-\mathrm{d} K \mu, \mathrm{d} \mu)=(\mathrm{d} \omega-S \mu, \mathrm{d} \mu) .
$$

By construction, $\mathcal{A} \circ \mathcal{A}=0$, and $\Phi$ is a cochain isomorphism from (11.6) to the complex

$$
0 \rightarrow \Lambda^{0}(\mathbb{W}) \stackrel{\mathcal{A}}{\rightarrow} \Lambda^{1}(\mathbb{W}) \stackrel{\mathcal{A}}{\rightarrow} \cdots \stackrel{\mathcal{A}}{\longrightarrow} \Lambda^{n}(\mathbb{W}) \rightarrow 0
$$

Using the definition of the exterior derivative, the definition of $K$, and the Leibniz rule, we obtain

$$
\left(S_{k} \omega\right)_{x}\left(v_{1}, \ldots, v_{k+1}\right)=\sum_{j=1}^{k+1}(-1)^{j+1} v_{j} \wedge \omega_{x}\left(v_{1}, \ldots, \hat{v}_{j}, \ldots, v_{k+1}\right),
$$

so $S_{k}$ is precisely the operator defined in (6.2) of Section 6, applied pointwise. As shown in that section, the operator $S_{n-2}: \Lambda^{n-2}(\Omega ; \mathbb{V}) \rightarrow \Lambda^{n-1}(\Omega ; \mathbb{K})$ is an isomorphism. This will be crucial to the construction.

To proceed, we define

$$
\begin{aligned}
& \Gamma^{n-2}=\left\{(\omega, \mu) \in \Lambda^{n-2}(\mathbb{W}) \mid \mathrm{d} \omega=S \mu\right\}, \\
& \Gamma^{n-1}=\left\{(\omega, \mu) \in \Lambda^{n-1}(\mathbb{W}) \mid \omega=0\right\},
\end{aligned}
$$

with projections $\pi^{n-2}: \Lambda^{n-2}(\mathbb{W}) \rightarrow \Gamma^{n-2}$ and $\pi^{n-1}: \Lambda^{n-1}(\mathbb{W}) \rightarrow \Gamma^{n-1}$ given by

$$
\pi^{n-2}(\omega, \mu)=\left(\omega, S_{n-2}^{-1} \mathrm{~d} \omega\right), \quad \pi^{n-1}(\omega, \mu)=\left(0, \mu+\mathrm{d} S_{n-2}^{-1} \omega\right) .
$$

Using (11.7), it is straightforward to check that $\mathcal{A}$ maps $\Lambda^{n-2}(\mathbb{W})$ into $\Gamma^{n-2}$ and $\Gamma^{n-2}$ into $\Gamma^{n-1}$, so that

$$
0 \rightarrow \Lambda^{0}(\mathbb{W}) \stackrel{\mathcal{A}}{\longrightarrow} \cdots \stackrel{\mathcal{A}}{\longrightarrow} \Lambda^{n-3}(\mathbb{W}) \stackrel{\mathcal{A}}{\longrightarrow} \Gamma^{n-2} \stackrel{\mathcal{A}}{\longrightarrow} \Gamma^{n-1} \stackrel{\mathcal{A}}{\rightarrow} \Lambda^{n}(\mathbb{W}) \rightarrow 0
$$


is a subcomplex of (11.8). Moreover, the diagram

$$
\begin{aligned}
& \cdots \stackrel{\mathcal{A}}{\longrightarrow} \Lambda^{n-3}(\mathbb{W}) \stackrel{\mathcal{A}}{\longrightarrow} \Lambda^{n-2}(\mathbb{W}) \stackrel{\mathcal{A}}{\longrightarrow} \Lambda^{n-1}(\mathbb{W}) \stackrel{\mathcal{A}}{\longrightarrow} \Lambda^{n}(\mathbb{W}) \rightarrow 0 \\
& \downarrow \text { id } \downarrow \pi^{n-2} \quad \downarrow \pi^{n-1} \quad \downarrow \text { id } \\
& \ldots \stackrel{\mathcal{A}}{\longrightarrow} \Lambda^{n-3}(\mathbb{W}) \stackrel{\mathcal{A}}{\longrightarrow} \quad \Gamma^{n-2} \stackrel{\mathcal{A}}{\longrightarrow} \quad \Gamma^{n-1} \quad \stackrel{\mathcal{A}}{\longrightarrow} \Lambda^{n}(\mathbb{W}) \rightarrow 0
\end{aligned}
$$

commutes, and as each of the vertical maps is a projection, they induce a surjective map on cohomology. Now we introduce the obvious isomorphisms

$$
\Lambda^{n-2}(\Omega ; \mathbb{K}) \cong \Gamma^{n-2}, \quad \omega \mapsto\left(\omega, S_{n-2}^{-1} \mathrm{~d} \omega\right),
$$

and

$$
\Lambda^{n-1}(\Omega ; \mathbb{V}) \cong \Gamma^{n-1}, \quad \mu \mapsto(0, \mu) .
$$

Then the bottom row of (11.9) becomes

$$
\begin{aligned}
0 \rightarrow \Lambda^{0}(\mathbb{W}) \stackrel{\mathcal{A}}{\longrightarrow} \cdots & \stackrel{\mathcal{A}}{\longrightarrow} \Lambda^{n-3}(\mathbb{W}) \stackrel{\left(\mathrm{d},-S_{n-3}\right)}{\longrightarrow} \Lambda^{n-2}(\Omega ; \mathbb{K}) \\
& \stackrel{\text { do } S_{n-2}^{-1} \text { od }}{\longrightarrow} \Lambda^{n-1}(\Omega ; \mathbb{V}) \stackrel{\left(-S_{n-1}, \mathrm{~d}\right)^{T}}{\longrightarrow} \Lambda^{n}(\mathbb{W}) \rightarrow 0
\end{aligned}
$$

We refer to the complex (11.10), or at least terms of degree $n-3$ through $n$, as the elasticity complex. Since the highest-order de Rham cohomology group of $\Omega$ vanishes, it follows from the construction that the same holds true for the highest-order cohomology group of the elasticity complex. The same is true for the $L^{2}$ version of the elasticity complex, and this is what is needed to show the well-posedness of the problem (11.4). We show this in Section 11.4.

\subsection{Connections to 2-D and 3-D elasticity complexes}

In this subsection, we consider the special cases $n=2$ and $n=3$ and identify the elasticity complex with a complex consisting of spaces of scalar-, vector-, and tensor-valued proxy fields, i.e., fields with values in $\mathbb{R}, \mathbb{V}=\mathbb{R}^{n}$, and $\mathbb{M}:=\mathbb{V} \otimes \mathbb{V}$, and mappings which are differential operators.

Our tools for making the identifications are simple:

(1) Algebraic 1-forms may be identified with vectors via the Riesz map $j$ induced by the inner product in $\mathbb{V}$. In this way $\operatorname{Alt}^{1} \mathbb{V} \cong \mathbb{V}$ and $\operatorname{Alt}^{1}(\mathbb{V} ; \mathbb{V}) \cong \mathbb{M}$.

(2) In $2 \mathrm{D}$ the Hodge star operation provides isomorphisms $\mathrm{Alt}^{2} \mathbb{V} \stackrel{\star}{\rightarrow} \mathbb{R}$ and $\mathrm{Alt}^{1} \mathbb{V} \stackrel{\star}{\rightarrow} \mathrm{Alt}^{1} \mathbb{V}$. In $3 \mathrm{D}$ it provides isomorphisms $\mathrm{Alt}^{3} \mathbb{V} \stackrel{\star}{\rightarrow} \mathbb{R}$ and $\mathrm{Alt}^{2} \mathbb{V} \stackrel{\star}{\rightarrow} \mathrm{Alt}^{1} \mathbb{V}$.

(3) In 2D the Hodge star operation for multivectors (which in this subsec- 
tion we denote $\circledast)$ provides an isomorphism from $\mathbb{K} \stackrel{\circledast}{\longrightarrow} \mathbb{R}$. In $3 \mathrm{D}$ the isomorphism is from $\mathbb{K} \stackrel{\circledast}{\longrightarrow} \mathbb{V}$.

If we use a positively oriented orthonormal basis $e_{i}$ and dual basis $x_{i}$, these isomorphisms are as follows.

(1) $\mathrm{d} x_{i} \stackrel{j}{\mapsto} e_{i}$ and $e_{j} \mathrm{~d} x_{i} \stackrel{j}{\mapsto} e_{i} \otimes e_{j}$.

(2) $2 \mathrm{D}: \mathrm{d} x_{1} \wedge \mathrm{d} x_{2} \stackrel{\star}{\mapsto} 1$ and $\mathrm{d} x_{1} \stackrel{\star}{\mapsto} \mathrm{d} x_{2}, \mathrm{~d} x_{2} \stackrel{\star}{\mapsto}-\mathrm{d} x_{1}$;

3D: $\mathrm{d} x_{1} \wedge \mathrm{d} x_{2} \wedge \mathrm{d} x_{3} \stackrel{\star}{\mapsto} 1$ and $\mathrm{d} x_{1} \wedge \mathrm{d} x_{2} \stackrel{\star}{\mapsto} \mathrm{d} x_{3}, \mathrm{~d} x_{1} \wedge \mathrm{d} x_{3} \stackrel{\star}{\mapsto}-\mathrm{d} x_{2}$, $\mathrm{d} x_{2} \wedge \mathrm{d} x_{3} \stackrel{\star}{\mapsto} \mathrm{d} x_{1}$.

(3) $2 \mathrm{D}: e_{1} \wedge e_{2} \stackrel{\circledast}{\longmapsto} 13 \mathrm{D}: e_{1} \wedge e_{2} \stackrel{\circledast}{\longmapsto} e_{3}$, etc.

As an example, we compute the operator $\mathbb{V} \rightarrow \mathbb{V}$ in two dimensions which corresponds to the map $S_{0}: \mathbb{V} \rightarrow \operatorname{Alt}^{1}(\mathbb{V} ; \mathbb{K})$ after identifying the last space with $\mathbb{V}$ via $\circledast$, $\star$, and $j$. Now

$$
\left(S_{0} e_{1}\right)(v)=v \wedge e_{1}=\left[\mathrm{d} x_{1}(v) e_{1}+\mathrm{d} x_{2}(v) e_{2}\right] \wedge e_{1}=-e_{1} \wedge e_{2} \mathrm{~d} x_{2}(v),
$$

so $S_{0} e_{1}=-e_{1} \wedge e_{2} \mathrm{~d} x_{2}$. Thus, after the identifications, we compute the image of $e_{1}$ as

$$
e_{1} \stackrel{S_{0}}{\longmapsto}-e_{1} \wedge e_{2} \mathrm{~d} x_{2} \stackrel{\circledast}{\longmapsto}-\mathrm{d} x_{2} \stackrel{*}{\longmapsto} \mathrm{d} x_{1} \stackrel{j}{\longmapsto} e_{1} .
$$

Similarly $e_{2} \mapsto e_{2}$. Thus, modulo these isomorphisms, the map $S_{0}$ is simply the identify $\mathbb{V} \rightarrow \mathbb{V}$.

The elasticity complex in two dimensions

In the case $n=2$, the elasticity complex is

$$
0 \rightarrow \Lambda^{0}(\Omega ; \mathbb{K}) \stackrel{\mathrm{d}_{0} \circ S_{0}^{-1} \circ \mathrm{d}_{0}}{\longrightarrow} \Lambda^{1}(\Omega ; \mathbb{V}) \stackrel{\left(-S_{1}, \mathrm{~d}_{1}\right)^{T}}{\longrightarrow} \Lambda^{2}(\Omega ; \mathbb{K}) \times \Lambda^{2}(\Omega ; \mathbb{V}) \rightarrow 0
$$

Using the listed isomorphisms, we can convert the spaces of differential forms to function spaces:

$$
\begin{aligned}
& \Lambda^{0}(\Omega ; \mathbb{K}) \stackrel{\circledast}{\rightarrow} \Lambda^{0}(\Omega)=C^{\infty}(\Omega) \\
& \Lambda^{1}(\Omega ; \mathbb{V}) \stackrel{\star}{\rightarrow} \Lambda^{1}(\Omega ; \mathbb{V}) \stackrel{j}{\rightarrow} C^{\infty}(\Omega ; \mathbb{M}) \\
& \Lambda^{2}(\Omega ; \mathbb{K}) \stackrel{\star}{\rightarrow} \Lambda^{0}(\Omega ; \mathbb{K})=C^{\infty}(\Omega ; \mathbb{K}) \\
& \Lambda^{2}(\Omega ; \mathbb{V}) \stackrel{\star}{\rightarrow} \Lambda^{0}(\Omega ; \mathbb{V})=C^{\infty}(\Omega ; \mathbb{V}) .
\end{aligned}
$$

In this way, the elasticity complex becomes

$$
0 \rightarrow C^{\infty}(\Omega) \stackrel{J}{\rightarrow} C^{\infty}(\Omega ; \mathbb{M}) \stackrel{(\text { skw,div })^{T}}{\longrightarrow} C^{\infty}(\Omega ; \mathbb{K}) \times C^{\infty}(\Omega ; \mathbb{V}) \rightarrow 0 .
$$

Indeed, we have already seen that the operator $S_{1}$ corresponds to a constant multiple of skw, and that the operator $\mathrm{d}_{1}$ corresponds to the tensor divergence (i.e., the map $v \otimes w \mapsto(\operatorname{div} v) \otimes w)$. So we need only clarify 
the operator $J$ which corresponds to $\mathrm{d}_{0} \circ S_{0}^{-1} \circ \mathrm{d}_{0}$. Identifying $\Lambda^{1}(\Omega ; \mathbb{K})$ with $C^{\infty}(\Omega ; \mathbb{V})$ via $\circledast$, , and $j$, we find that $\mathrm{d}_{0}: \Lambda^{0}(\Omega ; \mathbb{K}) \rightarrow \Lambda^{1}(\Omega ; \mathbb{K})$ corresponds, as usual, to the operator curl : $C^{\infty}(\Omega) \rightarrow C^{\infty}(\Omega ; \mathbb{V})$ and the operator $\mathrm{d}_{0}: \Lambda^{0}(\Omega ; \mathbb{V}) \rightarrow \Lambda^{1}(\Omega ; \mathbb{V})$ to the corresponding operator on vectors curl : $C^{\infty}(\Omega ; \mathbb{V}) \rightarrow C^{\infty}(\Omega ; \mathbb{M})$ given by $\operatorname{curl}\left(\phi e_{i}\right)=(\operatorname{curl} \phi) \otimes e_{i}$. Also, we have seen, modulo these identifications, that $S_{0}$ corresponds to the identity operator on $C^{\infty}(\Omega ; \mathbb{V})$. Thus we conclude that the operator $J$ corresponding to $\mathrm{d}_{0} \circ S_{0}^{-1} \circ \mathrm{d}_{0}$ is curl curl : $C^{\infty}(\Omega) \rightarrow C^{\infty}(\Omega ; \mathbb{M})$. Written in terms of the usual basis on $\mathbb{V}$, it is given by

$$
J \phi=\left(\begin{array}{cc}
\frac{\partial^{2} \phi}{\partial x_{2}^{2}} & -\frac{\partial^{2} \phi}{\partial x_{1} \partial x_{2}} \\
-\frac{\partial^{2} \phi}{\partial x_{1} \partial x_{2}} & \frac{\partial^{2} \phi}{\partial x_{1}{ }^{2}}
\end{array}\right) .
$$

The elasticity complex in three dimensions

When $n=3$, the elasticity complex (11.10) is

$$
\begin{aligned}
& 0 \rightarrow \Lambda^{0}(\Omega ; \mathbb{K}) \times \Lambda^{0}(\Omega ; \mathbb{V}) \stackrel{\left(\mathrm{d}_{0},-S_{0}\right)}{\longrightarrow} \Lambda^{1}(\Omega ; \mathbb{K}) \\
& \stackrel{\mathrm{d}_{1} \circ S_{1}^{-1} \circ \mathrm{d}_{1}}{\longrightarrow} \Lambda^{2}(\Omega ; \mathbb{V}) \stackrel{\left(-S_{2}, \mathrm{~d}\right)^{T}}{\longrightarrow} \Lambda^{3}(\Omega ; \mathbb{K}) \times \Lambda^{3}(\Omega ; \mathbb{V}) \rightarrow 0 .
\end{aligned}
$$

We shall show that this corresponds to

$$
\begin{aligned}
0 \rightarrow C^{\infty}(\Omega ; \mathbb{V}) \times C^{\infty}(\Omega ; \mathbb{K}) \stackrel{(\mathrm{grad}, i)}{\longrightarrow} C^{\infty}(\Omega ; \mathbb{M}) \\
\quad \stackrel{J}{\rightarrow} C^{\infty}(\Omega ; \mathbb{M}) \stackrel{(\mathrm{skw}, \mathrm{div})^{T}}{\longrightarrow} C^{\infty}(\Omega ; \mathbb{K}) \times C^{\infty}(\Omega ; \mathbb{V}) \rightarrow 0
\end{aligned}
$$

where the operator $i$ represents the inclusion of $\mathbb{K}$ into $\mathbb{M}$, and the operator $J$ is a second-order differential operator which will be defined below. More precisely, if the spaces in (11.11) are mapped isomorphically onto the corresponding spaces in (11.12) using the three classes of isomorphisms listed at the start of this subsection, then the maps in (11.11) correspond via composition to the maps shown in (11.12), up to nonzero constant factors.

The correspondence between the last parts of the sequences

$$
\Lambda^{2}(\Omega ; \mathbb{V}) \stackrel{\left(-S_{2}, \mathrm{~d}_{2}\right)^{T}}{\longrightarrow} \Lambda^{3}(\Omega ; \mathbb{K}) \times \Lambda^{3}(\Omega ; \mathbb{V})
$$

and

$$
C^{\infty}(\Omega ; \mathbb{M}) \stackrel{(\text { skw,div })^{T}}{\longrightarrow} C^{\infty}(\Omega ; \mathbb{K}) \times C^{\infty}(\Omega ; \mathbb{V})
$$

is straightforward. We have already seen that $S_{2}$ corresponds to a multiple of skw and that $\mathrm{d}_{2}$ corresponds to the tensor divergence.

The correspondence between the first parts of the sequences

$$
\Lambda^{0}(\Omega ; \mathbb{K}) \times \Lambda^{0}(\Omega ; \mathbb{V}) \stackrel{\left(\mathrm{d}_{0},-S_{0}\right)}{\longrightarrow} \Lambda^{1}(\Omega ; \mathbb{K})
$$


and

$$
C^{\infty}(\Omega ; \mathbb{V}) \times C^{\infty}(\Omega ; \mathbb{K}) \stackrel{(\mathrm{grad}, i)}{\longrightarrow} C^{\infty}(\Omega ; \mathbb{M})
$$

is similar. It is easy to see that $\mathrm{d}_{0}$ corresponds to the vector gradient, and we can follow the basis elements through the mappings to see that $S_{0}$ corresponds to the inclusion $i$. For example, on the basis element $e_{1} \wedge e_{2}=$ $e_{1} \otimes e_{2}-e_{2} \otimes e_{1}$, we get

$e_{1} \wedge e_{2} \stackrel{\circledast}{\longmapsto} e_{3} \stackrel{S_{0}}{\longmapsto} e_{1} \wedge e_{3} \mathrm{~d} x_{1}+e_{2} \wedge e_{3} \mathrm{~d} x_{2} \stackrel{\circledast}{\longmapsto}-e_{2} \mathrm{~d} x_{1}+e_{1} \mathrm{~d} x_{2} \stackrel{j}{\longmapsto} e_{1} \otimes e_{2}-e_{2} \otimes e_{1}$.

In order to identify the operator $J$ corresponding to $\mathrm{d}_{1} \circ S_{1}^{-1} \circ \mathrm{d}_{1}$, consider the diagram

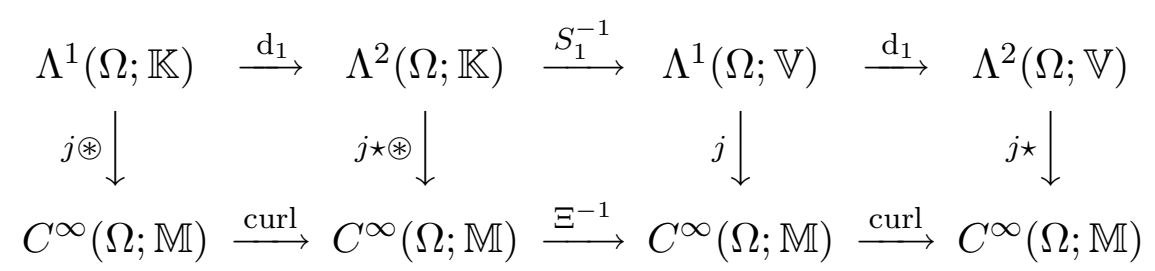

We have indicated the operators corresponding to the two occurrences of $\mathrm{d}_{1}$. As is easy to check, they are both occurrences of the tensor curl, $f \otimes e_{i} \mapsto$ curl $f \otimes e_{i}$ for any smooth vector field $f$. We have also denoted the operator corresponding to $S_{1}$, namely $j \star \circledast S_{1} j^{-1}$, by $\Xi$. We now compute $\Xi$ using a basis. Since

$$
\begin{aligned}
S_{1}\left(e_{1} \mathrm{~d} x_{1}\right)(v, w)= & v \wedge e_{1} \mathrm{~d} x_{1}(w)-w \wedge e_{1} \mathrm{~d} x_{1}(v) \\
= & e_{2} \wedge e_{1} \mathrm{~d} x_{2}(v) \mathrm{d} x_{1}(w)+e_{3} \wedge e_{1} \mathrm{~d} x_{3}(v) \mathrm{d} x_{1}(w) \\
& -e_{2} \wedge e_{1} \mathrm{~d} x_{2}(w) \mathrm{d} x_{1}(v)-e_{3} \wedge e_{1} \mathrm{~d} x_{3}(w) \mathrm{d} x_{1}(v) \\
= & \left(e_{1} \wedge e_{2} \mathrm{~d} x_{1} \wedge \mathrm{d} x_{2}+e_{1} \wedge e_{3} \mathrm{~d} x_{1} \wedge \mathrm{d} x_{3}\right)(v, w),
\end{aligned}
$$

we have

$$
S_{1}\left(e_{1} \mathrm{~d} x_{1}\right)=e_{1} \wedge e_{2} \mathrm{~d} x_{1} \wedge \mathrm{d} x_{2}+e_{1} \wedge e_{3} \mathrm{~d} x_{1} \wedge \mathrm{d} x_{3} .
$$

A similar computation gives

$$
S_{1}\left(e_{1} \mathrm{~d} x_{2}\right)=e_{1} \wedge e_{3} \mathrm{~d} x_{2} \wedge \mathrm{d} x_{3} .
$$

Thus $\Xi\left(e_{1} \otimes e_{1}\right)$ is the composition

$$
\begin{aligned}
& e_{1} \otimes e_{1} \stackrel{j^{-1}}{\longmapsto} e_{1} \mathrm{~d} x_{1} \stackrel{S_{1}}{\longmapsto} e_{1} \wedge e_{2} \mathrm{~d} x_{1} \wedge \mathrm{d} x_{2}+e_{1} \wedge e_{3} \mathrm{~d} x_{1} \wedge \mathrm{d} x_{3} \\
& \stackrel{\circledast \star}{\longmapsto} e_{3} \mathrm{~d} x_{3}+e_{2} \mathrm{~d} x_{2} \stackrel{j}{\longmapsto} e_{2} \otimes e_{2}+e_{3} \otimes e_{3},
\end{aligned}
$$

and $\Xi\left(e_{2} \otimes e_{1}\right)=-e_{1} \otimes e_{2}$. Since similar expressions apply to the other basis functions, we find that

$$
\Xi\left(e_{i} \otimes e_{j}\right)=\delta_{i j} \sum_{k} e_{k} \otimes e_{k}-e_{j} \otimes e_{i}
$$


for all $i$, $j$, or, equivalently, $\Xi F=\operatorname{tr}(F) I-F^{T}$ for $F \in \mathbb{M}$. We then have $\Xi^{-1} F=(1 / 2) \operatorname{tr}(F) I-F^{T}$, and

$$
J F=\operatorname{curl}(\Xi \operatorname{curl} F), \quad F \in C^{\infty}(\Omega ; \mathbb{M}) .
$$

It is worth remarking that if $F=F^{T}$, then $J F=\operatorname{curl}(\operatorname{curl} F)^{T}$, and if $F=-F^{T}$ then $J F=0$.

There are also elasticity complexes corresponding to the case of strongly imposed symmetry. In two dimensions, this complex takes the form

$$
0 \rightarrow C^{\infty} \stackrel{J}{\rightarrow} C^{\infty}(\Omega ; \mathbb{S}) \stackrel{\operatorname{div}}{\longrightarrow} C^{\infty}(\Omega ; \mathbb{V}) \rightarrow 0,
$$

where $\mathbb{S} \subset \mathbb{M}$ is the space of symmetric tensors. The complex (11.13) can be obtained from the complex (11.13) by performing a projection step. To see this, consider the diagram

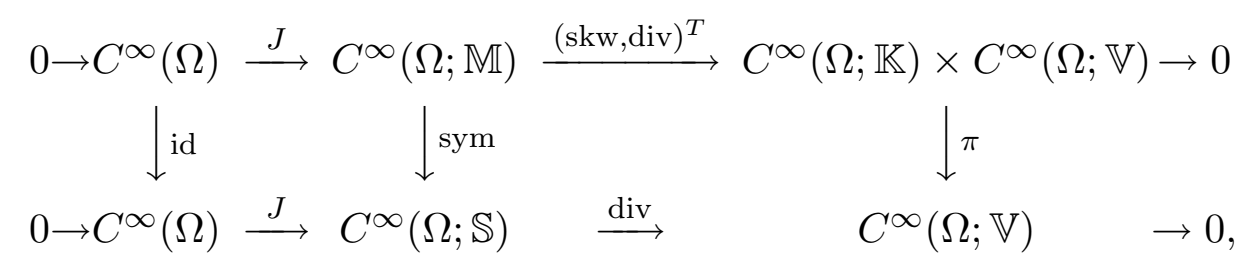

$\pi(q, u)=u-\operatorname{div} q$. The vertical maps are projections onto subspaces and the diagram commutes, so define a cochain projection, and therefore induce a surjection on cohomology. The connection between the two versions of the elasticity complex is explored in more detail in Arnold et al. (2006c), but will not be pursued here.

The corresponding elasticity complex in three dimensions with strongly imposed symmetry of the stress tensor is given by

$$
0 \rightarrow C^{\infty}(\Omega ; \mathbb{V}) \stackrel{\epsilon}{\rightarrow} C^{\infty}(\Omega ; \mathbb{S}) \stackrel{J}{\rightarrow} C^{\infty}(\Omega ; \mathbb{S}) \stackrel{\operatorname{div}}{\rightarrow} C^{\infty}(\Omega ; \mathbb{V}) \rightarrow 0,
$$

where $\epsilon u$ is the symmetric part of $\operatorname{grad} u$ for a vector field $u$. If we were to follow the program outlined previously for mixed methods for the Poisson equation, the construction of stable mixed finite elements for elasticity for strong symmetry would be based on extending the sequence (11.13) to a complete commuting diagram of the form

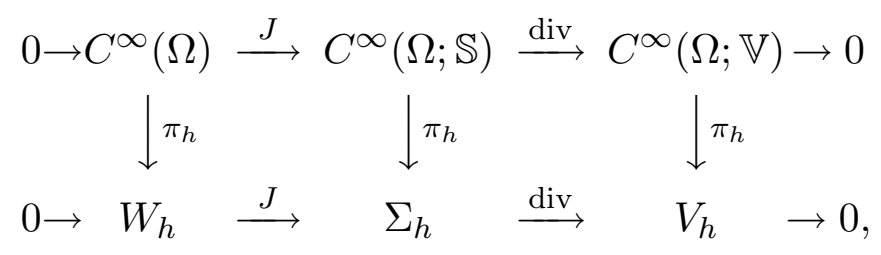

where $W_{h} \subset H^{2}(\Omega), \Sigma_{h} \subset H(\operatorname{div}, \Omega ; \mathbb{S})$ and $V_{h} \subset L^{2}(\Omega ; \mathbb{V})$ are suitable finite element spaces and the $\pi_{h}^{0}$ are corresponding projection operators defining a cochain projection. This is exactly the construction performed in Arnold and Winther (2002) in two dimensions. In particular, since the finite 
element space $W_{h}$ is required to be a subspace of $H^{2}(\Omega)$ (the natural domain of $J$ ), we can conclude that the finite element space $W_{h}$ must contain quintic polynomials, and therefore the lowest-order space $\Sigma_{h}$ will at least involve piecewise cubics. In fact, for the lowest-order elements discussed in Arnold and Winther (2002), $W_{h}$ is the classical Argyris space, while $\Sigma_{h}$ consists of piecewise cubic symmetric tensor fields with a linear divergence.

The analogous approach in three dimensions would be based on developing finite element spaces approximating the spaces in the complex (11.14) and embedding (11.14) as the top row of a commuting diagram analogous to (11.15), with a corresponding finite element complex as the bottom row. However, as mentioned previously, when the symmetry constraint is enforced pointwise on the discrete stress space, this construction leads to intricate finite elements of high-order. In this paper, we instead pursue an approach based on the weak symmetry formulation.

\subsection{Well-posedness of the continuous problem}

As discussed in Section 11.1, to establish well-posedness of the elasticity problem with weakly imposed symmetry (11.4), it suffices to verify condition (W2) of that subsection. This follows from the following theorem, which says that the map

$$
H \Lambda^{n-1}(\Omega ; \mathbb{V}) \stackrel{(-S, \mathrm{~d})^{T}}{\longrightarrow} H \Lambda^{n}(\Omega ; \mathbb{K}) \times H \Lambda^{n}(\Omega ; \mathbb{V})
$$

is surjective, i.e., that the highest-order cohomology of the $L^{2}$ elasticity sequence vanishes. We spell out the proof in detail, since it will give us guidance as we construct stable discretizations.

Theorem 11.1. Given $(\omega, \mu) \in L^{2} \Lambda^{n}(\Omega ; \mathbb{K}) \times L^{2} \Lambda^{n}(\Omega ; \mathbb{V})$, there exists $\sigma \in H \Lambda^{n-1}(\Omega ; \mathbb{V})$ such that $\mathrm{d} \sigma=\mu,-S_{n-1} \sigma=\omega$. Moreover, we may choose $\sigma$ so that

$$
\|\sigma\|_{H \Lambda} \leq c(\|\omega\|+\|\mu\|)
$$

for a fixed constant $c$.

Proof. The second sentence follows from the first by Banach's theorem, so we need only prove the first.

(1) By Theorem 2.4, we can find $\eta \in H^{1} \Lambda^{n-1}(\Omega ; \mathbb{V})$ with $\mathrm{d} \eta=\mu$.

(2) Since $\omega+S_{n-1} \eta \in H \Lambda^{n}(\Omega ; K)$, we can apply Theorem 2.4 a second time to find $\tau \in H^{1} \Lambda^{n-1}(\Omega ; \mathbb{K})$ with $\mathrm{d} \tau=\omega+S_{n-1} \eta$.

(3) Since $S_{n-2}$ is an isomorphism from $\operatorname{Alt}^{n-2}(\mathbb{V} ; \mathbb{V})$ to $\operatorname{Alt}^{n-1}(\mathbb{V} ; \mathbb{K})$, when applied pointwise, it gives an isomorphism of the space $H^{1} \Lambda^{n-2}(\Omega ; \mathbb{V})$ onto $H^{1} \Lambda^{n-1}(\Omega ; \mathbb{K})$, and so we have $\rho \in H^{1} \Lambda^{n-2}(\Omega ; \mathbb{V})$ with $S_{n-2} \rho=$ $\tau$.

(4) Define $\sigma=\mathrm{d} \rho+\eta \in H \Lambda^{n-1}(\Omega ; \mathbb{V})$. 
(5) From steps (1) and (4), it is immediate that $\mathrm{d} \sigma=\mu$.

(6) From (4), $-S_{n-1} \sigma=-S_{n-1} \mathrm{~d} \rho-S_{n-1} \eta$. But, since $\mathrm{d} S=-S \mathrm{~d}$,

$$
-S_{n-1} \mathrm{~d} \rho=\mathrm{d} S_{n-2} \rho=\mathrm{d} \tau=\omega+S_{n-1} \eta,
$$

so $-S_{n-1} \sigma=\omega$.

We note a few points from the proof. First, although the elasticity problem (11.4) only involves the three spaces $H \Lambda^{n-1}(\Omega ; \mathbb{V}), L^{2} \Lambda^{n}(\Omega ; \mathbb{V})$, and $L^{2} \Lambda^{n}(\Omega ; \mathbb{K})$, the proof brings in two additional spaces from the BGG construction: $H \Lambda^{n-2}(\Omega ; \mathbb{V})$ and $H \Lambda^{n-1}(\Omega ; \mathbb{K})$. Also, although $S_{n-1}$ is the only $S$ operator arising in the formulation, $S_{n-2}$ plays a role in the proof. Note, however, that we do not fully use the fact that $S_{n-2}$ is an isomorphism from $\operatorname{Alt}^{n-2}(\mathbb{V} ; \mathbb{V})$ to $\operatorname{Alt}^{n-1}(\mathbb{V} ; \mathbb{K})$, only the fact that it is a surjection. This will prove important in the next subsection, when we construct a discrete elasticity complex.

\subsection{A discrete elasticity complex}

In this subsection, we derive a discrete version of the elasticity sequence by adapting the construction of Section 11.2. To carry out the construction, we will use two discretizations of the de Rham sequence, in general different, one to discretize the $\mathbb{K}$-valued de Rham sequence and one the $\mathbb{V}$-valued de Rham sequence. For $k=0,1, \ldots, n$, let $\Lambda_{h}^{k}$ define a finite-dimensional subcomplex of the $L^{2}$ de Rham complex with an associated cochain projection $\Pi_{h}^{k}$ : $\Lambda^{k} \rightarrow \Lambda_{h}^{k}$. Thus the following diagram commutes:

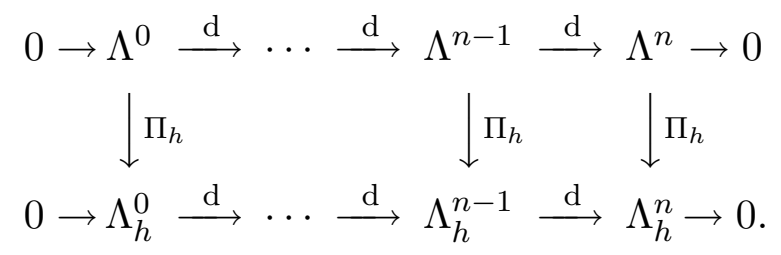

We do not make a specific choice of the discrete spaces yet, but, as shown in Section 5, there exist many such complexes based on the spaces $\mathcal{P}_{r} \Lambda^{k}\left(\mathcal{T}_{h}\right)$ and $\mathcal{P}_{r}^{-} \Lambda^{k}\left(\mathcal{T}_{h}\right)$ for a simplicial decomposition $\mathcal{T}_{h}$ of $\Omega$. In fact, for each polynomial degree $r \geq 0$ there exists $2^{n-1}$ such complexes with $\Lambda_{h}^{n}=\mathcal{P}_{r} \Lambda^{n}\left(\mathcal{T}_{h}\right)$.

Let $\bar{\Lambda}_{h}^{k}$ denote a second finite-dimensional subcomplex of the $L^{2}$ de Rham complex with a corresponding cochain projection $\bar{\Pi}_{h}$ enjoying the same properties. Supposing a compatibility condition between these two discretizations, which we describe below, we shall construct a discrete elasticity complex based on them, in close analogy with the BGG construction in Section 11.2.

Let $\Lambda_{h}^{k}(\mathbb{K})=\Lambda_{h}^{k} \otimes \mathbb{K}$ and $\bar{\Lambda}_{h}^{k}(\mathbb{V})=\bar{\Lambda}_{h}^{k} \otimes \mathbb{V}$. For brevity, we write $\Lambda_{h}^{k}(\mathbb{W})$ 
for $\Lambda_{h}^{k}(\mathbb{K}) \times \bar{\Lambda}_{h}^{k}(\mathbb{V})$. In analogy with (11.6), we start with the complex

$$
0 \rightarrow \Lambda_{h}^{0}(\mathbb{W}) \stackrel{\left(\begin{array}{ll}
\mathrm{d} & 0 \\
0 & \mathrm{~d}
\end{array}\right)}{\longrightarrow} \Lambda_{h}^{1}(\mathbb{W}) \stackrel{\left(\begin{array}{ll}
\mathrm{d} & 0 \\
0 & \mathrm{~d}
\end{array}\right)}{\longrightarrow} \cdots \stackrel{\left(\begin{array}{ll}
\mathrm{d} & 0 \\
0 & \mathrm{~d}
\end{array}\right)}{\longrightarrow} \Lambda_{h}^{n}(\mathbb{W}) \rightarrow 0 .
$$

Since $\bar{\Lambda}_{h}^{k}$ may not equal $\Lambda_{h}^{k}$, the operator $K$ may not map $\Lambda_{h}^{k}(\mathbb{K})$ into $\bar{\Lambda}_{h}^{k}(\mathbb{V})$. So we define $K_{h}: \bar{\Lambda}_{h}^{k}(\mathbb{V}) \rightarrow \Lambda_{h}^{k}(\mathbb{K})$ by $K_{h}=\Pi_{h} K$ where $\Pi_{h}$ is the given projection operator onto $\Lambda_{h}^{k}(\mathbb{K})$.

Next we define $S_{h}=S_{k, h}: \bar{\Lambda}_{h}^{k}(\mathbb{V}) \rightarrow \Lambda_{h}^{k+1}(\mathbb{K})$ by $S_{h}=\mathrm{d} K_{h}-K_{h} \mathrm{~d}$, for $k=0,1, \ldots, n-1$. Observe that the discrete version of (11.7),

$$
\mathrm{d} S_{h}=-S_{h} \mathrm{~d}
$$

follows (exactly as in the continuous case) from $\mathrm{d}^{2}=0$. From the commutative diagram (11.16), we see that

$$
S_{h}=\mathrm{d} \Pi_{h} K-\Pi_{h} K \mathrm{~d}=\Pi_{h}(\mathrm{~d} K-K \mathrm{~d})=\Pi_{h} S .
$$

Continuing to mimic the continuous case, we define the automorphism $\Phi_{h}$ on $\Lambda_{h}^{k}(\mathbb{W})$ by

$$
\Phi_{h}(\omega, \mu)=\left(\omega+K_{h} \mu, \mu\right),
$$

and the operator $\mathcal{A}_{h}: \Lambda_{h}^{k}(\mathbb{W}) \rightarrow \Lambda_{h}^{k+1}(\mathbb{W})$ by $\mathcal{A}_{h}=\Phi_{h} \mathrm{~d} \Phi_{h}^{-1}$, which leads to

$$
\mathcal{A}_{h}(\omega, \mu)=\left(\mathrm{d} \omega-S_{h} \mu, \mathrm{d} \mu\right)
$$

Inserting the isomorphisms $\Phi_{h}$ into (11.17), we obtain the isomorphic complex

$$
0 \rightarrow \Lambda_{h}^{0}(\mathbb{W}) \stackrel{\mathcal{A}_{h}}{\longrightarrow} \Lambda_{h}^{1}(\mathbb{W}) \stackrel{\mathcal{A}_{h}}{\longrightarrow} \cdots \stackrel{\mathcal{A}_{h}}{\longrightarrow} \Lambda_{h}^{n}(\mathbb{W}) \rightarrow 0 .
$$

As in the continuous case, the discrete elasticity complex will be realized as a subcomplex of this complex. We define

$$
\begin{aligned}
& \Gamma_{h}^{n-2}=\left\{(\omega, \mu) \in \Lambda_{h}^{n-2}(\mathbb{W}) \mid \mathrm{d} \omega=S_{n-2, h} \mu\right\}, \\
& \Gamma_{h}^{n-1}=\left\{(\omega, \mu) \in \Lambda_{h}^{n-1}(\mathbb{W}) \mid \omega=0\right\} .
\end{aligned}
$$

Again, $\mathcal{A}_{h}$ maps $\Lambda_{h}^{n-2}(\mathbb{W})$ into $\Gamma_{h}^{n-2}$ and $\Gamma_{h}^{n-2}$ into $\Gamma_{h}^{n-1}$, so that

$$
0 \rightarrow \Lambda_{h}^{0}(\mathbb{W}) \stackrel{\mathcal{A}_{h}}{\longrightarrow} \cdots \stackrel{\mathcal{A}_{h}}{\longrightarrow} \Lambda_{h}^{n-3}(\mathbb{W}) \stackrel{\mathcal{A}_{h}}{\longrightarrow} \Gamma_{h}^{n-2} \stackrel{\mathcal{A}_{h}}{\longrightarrow} \Gamma_{h}^{n-1} \stackrel{\mathcal{A}_{h}}{\longrightarrow} \Lambda_{h}^{n}(\mathbb{W}) \rightarrow 0
$$

is indeed a subcomplex of (11.19).

As in the continuous case, we could identify $\Gamma_{h}^{n-1}$ with $\bar{\Lambda}_{h}^{n-1}(\mathbb{V})$, but, unlike in the continuous case, we cannot identify $\Gamma_{h}^{n-2}$ with $\Lambda_{h}^{n-2}(\mathbb{K})$, since we do not require that $S_{n-2, h}$ be invertible (and it is in fact not invertible in the applications). However, we saw in the proof of Theorem 11.1, that the decisive property of $S_{n-2}$ is that it be surjective, and surjectivity of $S_{n-2, h}$ is what we shall require in order to derive a cochain projection and obtain 
the analogue of the diagram (11.9). Thus we make the following Surjectivity assumption:

The operator $S_{n-2, h}$ maps $\bar{\Lambda}_{h}^{n-2}(\mathbb{V})$ onto $\Lambda_{h}^{n-1}(\mathbb{K})$.

Under this assumption, the operator $S_{h}=S_{n-2, h}$ has a right inverse $S_{h}^{\dagger}$ mapping $\Lambda_{h}^{n-1}(\mathbb{K})$ into $\Lambda_{h}^{n-2}(\mathbb{V})$. This allows us to define discrete counterparts of the projection operators $\pi^{n-2}$ and $\pi^{n-1}$ by

$$
\pi_{h}^{n-2}(\omega, \mu)=\left(\omega, \mu-S_{h}^{\dagger} S_{h} \mu+S_{h}^{\dagger} \mathrm{d} \omega\right), \quad \pi_{h}^{n-1}(\omega, \mu)=\left(0, \mu+\mathrm{d} S_{h}^{\dagger} \omega\right),
$$

and obtain the discrete analogue of (11.9):

$$
\begin{aligned}
& \cdots \stackrel{\mathcal{A}_{h}}{\longrightarrow} \Lambda_{h}^{n-3}(\mathbb{W}) \stackrel{\mathcal{A}_{h}}{\longrightarrow} \Lambda_{h}^{n-2}(\mathbb{W}) \stackrel{\mathcal{A}_{h}}{\longrightarrow} \Lambda_{h}^{n-1}(\mathbb{W}) \stackrel{\mathcal{A}_{h}}{\longrightarrow} \Lambda_{h}^{n}(\mathbb{W}) \rightarrow 0 \\
& \downarrow \text { id } \downarrow \pi^{n-2} \quad \downarrow \pi^{n-1} \quad \downarrow \text { id } \\
& \ldots \stackrel{\mathcal{A}_{h}}{\longrightarrow} \Lambda_{h}^{n-3}(\mathbb{W}) \stackrel{\mathcal{A}_{h}}{\longrightarrow} \quad \Gamma_{h}^{n-2} \quad \stackrel{\mathcal{A}_{h}}{\longrightarrow} \Gamma_{h}^{n-1} \quad \stackrel{\mathcal{A}_{h}}{\longrightarrow} \Lambda_{h}^{n}(\mathbb{W}) \rightarrow 0 .
\end{aligned}
$$

It is straightforward to check that this diagram commutes. For example, if $(\omega, \mu) \in \Lambda_{h}^{n-3}(\mathbb{W})$, then

$$
\begin{aligned}
\pi_{h}^{n-2} \mathcal{A}_{h}(\omega, \mu) & =\pi_{h}^{n-2}\left(\mathrm{~d} \omega-S_{h} \mu, \mathrm{d} \mu\right) \\
& =\left(\mathrm{d} \omega-S_{h} \mu, \mathrm{d} \mu-S_{h}^{\dagger} S_{h} \mathrm{~d} \mu+S_{h}^{\dagger} d\left(\mathrm{~d} \omega-S_{h} \mu\right)\right) \\
& =\left(\mathrm{d} \omega-S_{h} \mu, \mathrm{d} \mu-S_{h}^{\dagger}\left(S_{h} \mathrm{~d} \mu+\mathrm{d} S_{h} \mu\right)\right)=\mathcal{A}_{h}(\omega, \mu),
\end{aligned}
$$

where the last equality follows from (11.18). Thus the vertical maps in (11.21) indeed define a cochain projection.

Since $\mathcal{A}_{h}$ maps $\Lambda_{h}^{n-1}(\mathbb{W})$ onto $\Lambda_{h}^{n}(\mathbb{W})$, the diagram implies that $\mathcal{A}_{h}$ maps $\Gamma_{h}^{n-1}$ onto $\Lambda_{h}^{n}(\mathbb{W})$, i.e., that $\left(-S_{n-1, h}, \mathrm{~d}\right)^{T} \operatorname{maps} \bar{H} \Lambda_{h}^{n-1}(\mathbb{V})$ onto $\Lambda_{h}^{n}(\mathbb{K}) \times$ $\bar{\Lambda}_{h}^{n}(\mathbb{V})$. This suggests that the choice of finite element spaces $\bar{H} \Lambda_{h}^{n-1}(\mathbb{V})$ for stress, $\bar{\Lambda}_{h}^{n}(\mathbb{V})$ for displacements, and $\Lambda_{h}^{n}(\mathbb{K})$ for the multiplier will lead to a stable discretization of (11.5). We now make specific choices for the two sets of spaces $\Lambda_{h}^{k}$ and $\bar{\Lambda}_{h}^{k}$ for $k=0,1, \ldots, n$ and verify the surjectivity assumption. Then in the next subsection we prove that they do, in fact, lead to a stable discretization.

Let $\mathcal{T}_{h}$ denote a family of shape-regular simplicial meshes of $\Omega$ indexed by $h$, the maximal diameter of the simplices in $\mathcal{T}_{h}$, and fix the degree $r \geq 0$. Our choices are then:

- $\Lambda_{h}^{n-1}=\mathcal{P}_{r+1}^{-} \Lambda^{n-1}\left(\mathcal{T}_{h}\right), \Lambda_{h}^{n}=\mathcal{P}_{r} \Lambda^{n}\left(\mathcal{T}_{h}\right)$, and

$$
\text { - } \bar{\Lambda}_{h}^{n-2}=\mathcal{P}_{r+2}^{-} \Lambda^{n-2}\left(\mathcal{T}_{h}\right), \bar{\Lambda}_{h}^{n-1}=\mathcal{P}_{r+1} \Lambda^{n-1}\left(\mathcal{T}_{h}\right), \bar{\Lambda}_{h}^{n}=\mathcal{P}_{r} \Lambda_{h}^{n}\left(\mathcal{T}_{h}\right) \text {. }
$$

For the remaining spaces, we choose $\Lambda_{h}^{k}$ and $\bar{\Lambda}_{h}^{k}$ as either $\mathcal{P}_{s+1}^{-} \Lambda^{k}\left(\mathcal{T}_{h}\right)$ or $\mathcal{P}_{s} \Lambda^{k}\left(\mathcal{T}_{h}\right)$, for appropriate degrees $s$, so as to obtain the commuting diagram (11.16). In all cases we use the canonical projection operator related to the degrees of freedom in the space, as defined at the end of Section 5.1. Note 
that in the lowest-order case $r=0$, we are approximating the stresses by piecewise linear functions and the displacements and the multiplier by piecewise constants.

We now verify the surjectivity assumption for this choice.

Theorem 11.2. Let $\Pi_{h}^{n-1}: \Lambda_{h}^{n-1}(\Omega ; \mathbb{K}) \rightarrow \mathcal{P}_{r+1}^{-} \Lambda^{n-1}\left(\mathcal{T}_{h} ; \mathbb{K}\right)$ and $\bar{\Pi}_{h}^{n-2}$ : $\Lambda^{n-2}(\Omega ; \mathbb{V}) \rightarrow \mathcal{P}_{r+2}^{-} \Lambda^{n-2}\left(\mathcal{T}_{h} ; \mathbb{V}\right)$ be the canonical projection operators defined in terms of the degrees of freedom (5.2). Then

$$
\Pi_{h}^{n-1} S_{n-2} \bar{\Pi}_{h}^{n-2}=\Pi_{h}^{n-1} S_{n-2} \text { on } \Lambda^{n-2}(\Omega ; \mathbb{V}) .
$$

Consequently $S_{n-2, h}:=\Pi_{h}^{n-1} S_{n-2}$ maps the space $\mathcal{P}_{r+2}^{-} \Lambda^{n-2}\left(\mathcal{T}_{h} ; \mathbb{V}\right)$ onto $\mathcal{P}_{r+1}^{-} \Lambda^{n-1}\left(\mathcal{T}_{h} ; \mathbb{K}\right)$.

Proof. We will show that the statement easily follows from the first, since $\Pi_{h}^{n-1}$ and $S_{n-2}$ are both surjective.

For the proof, we define the operator $K^{\prime}: \Lambda^{k}(\Omega ; \mathbb{K}) \rightarrow \Lambda^{k}(\Omega ; \mathbb{V})$ by

$$
\left(K^{\prime} \omega\right)_{x}\left(v_{1}, \ldots, v_{k}\right)=\omega_{x}\left(v_{1}, \ldots, v_{k}\right) X(x)
$$

where $X(x)$ is the element of $\mathbb{V}$ corresponding to $x$ and the last product is the action of the skew-symmetric operator $\omega_{x}\left(v_{1}, \ldots, v_{k}\right)$ on the vector $X(x)$. We then have

$$
K \omega \wedge \mu=\omega \wedge K^{\prime} \mu, \quad \omega \in \Lambda^{k}(\Omega ; \mathbb{V}), \mu \in \Lambda^{j}(\Omega ; \mathbb{K}) .
$$

We next show that

$$
S \omega \wedge \mu=(-1)^{k+1} \omega \wedge\left(K^{\prime} d-d K^{\prime}\right) \mu, \quad \omega \in \Lambda^{k}(\Omega ; \mathbb{V}), \mu \in \Lambda^{j}(\Omega ; \mathbb{K}) .
$$

This follows from the Leibniz rule. We have

$$
d K \omega \wedge \mu=\mathrm{d}(K \omega \wedge \mu)-(-1)^{k} K \omega \wedge \mathrm{d} \mu=\mathrm{d}\left(\omega \wedge K^{\prime} \mu\right)-(-1)^{k} \omega \wedge K^{\prime} \mathrm{d} \mu,
$$

and

$$
K \mathrm{~d} \omega \wedge \mu=\mathrm{d} \omega \wedge K^{\prime} \mu=\mathrm{d}\left(\omega \wedge K^{\prime} \mu\right)-(-1)^{k} \omega \wedge d K^{\prime} \mu .
$$

Subtracting we get (11.22). Thus, if $\mu \in \mathcal{P}_{r} \Lambda^{j}(\Omega ; \mathbb{K})$, there exists $\zeta \in$ $\mathcal{P}_{r-1} \Lambda^{j+1}(\Omega ; \mathbb{V})$ such that

$$
S \omega \wedge \mu= \pm \omega \wedge \zeta, \quad \omega \in \Lambda^{k}(\Omega ; \mathbb{K})
$$

(namely, just take $\left.\zeta=\left(K^{\prime} d-d K^{\prime}\right) \mu\right)$.

Now, to prove the theorem, we must show that

$$
\left(\Pi_{h}^{n-1} S_{n-2}-\Pi_{h}^{n-1} S_{n-2} \bar{\Pi}_{h}^{n-2}\right) \sigma=0
$$

for all $\sigma \in \Lambda^{n-2}(\Omega ; \mathbb{V})$. Defining $\omega=\left(I-\Pi_{h}^{n-2}\right) \sigma$, the required condition 
becomes $\Pi_{h}^{n-1} S_{n-2} \omega=0$. Since $\bar{\Pi}_{h}^{n-2} \omega=0$, we have

$$
\int_{f} \operatorname{Tr}_{f} \omega \wedge \zeta=0, \zeta \in \mathcal{P}_{r-d+n-1} \Lambda^{d-n+2}(f ; \mathbb{V}), f \in \Delta_{d}\left(\mathcal{T}_{h}\right), n-1 \leq d \leq n
$$

(in fact (11.23) holds for $\mathrm{d}=n-2$ as well, but this is not used here). We must show that (11.23) implies

$$
\int_{f} \operatorname{Tr}_{f}\left(S_{n-2} \omega\right) \wedge \mu=0
$$

for $\mu \in \mathcal{P}_{r-d+n-1} \Lambda^{d-n+1}(f ; \mathbb{K}), f \in \Delta_{d}\left(\mathcal{T}_{h}\right), n-1 \leq d \leq n$. This follows in view of the result proved in the last paragraph (applied on the face $f$; note that d, $K, K^{\prime}$ and $S$ commute with traces).

In the next subsection, we use this result to verify that this choice of spaces results in a stable finite element discretization of the variational formulation of elasticity with weak symmetry.

\subsection{The main stability result for mixed finite elements for elasticity}

We show in this subsection that the choices

$$
\begin{aligned}
\Lambda_{h}^{n-1}(\mathbb{V}) & =\mathcal{P}_{r+1} \Lambda^{n-1}\left(\mathcal{T}_{h} ; \mathbb{V}\right), \\
\Lambda_{h}^{n}(\mathbb{V}) & =\mathcal{P}_{r} \Lambda^{n}\left(\mathcal{T}_{h} ; \mathbb{V}\right), \\
\Lambda_{h}^{n}(\mathbb{K}) & =\mathcal{P}_{r} \Lambda^{n}\left(\mathcal{T}_{h} ; \mathbb{K}\right),
\end{aligned}
$$

give a stable finite element discretization of the system (11.5).

The first stability condition (S1) is obvious since, by construction,

$$
\mathrm{d} \mathcal{P}_{r+1} \Lambda^{n-1}\left(\mathcal{T}_{h} ; \mathbb{V}\right) \subset \mathcal{P}_{r} \Lambda^{n}\left(\mathcal{T}_{h} ; \mathbb{V}\right)
$$

The condition (S2) is more subtle. Our proof is inspired by the proof of the well-posedness result, Theorem 11.1, but involves a variety of projections from the continuous to the finite element spaces, and keeping track of norms. A technical difficulty arises because the canonical projection $\bar{\Pi}_{h}^{n-2}: \Lambda^{n-2}(\Omega ; \mathbb{V}) \rightarrow \mathcal{P}_{r+2}^{-} \Lambda^{n-2}\left(\mathcal{T}_{h} ; \mathbb{V}\right)$ is not bounded on $H^{1}$, since its definition involves traces on subsimplices of codimension 2. On the other hand, we cannot use the smoothed projection operators introduced in Section 5.4, because these do not preserve the moments of traces on faces of codimension 0 and 1 , which were required in the previous theorem to prove that $\Pi_{h}^{n-1} S_{n-2} \bar{\Pi}_{h}^{n-2}=\Pi_{h}^{n-1} S_{n-2}$. Hence we introduce a new operator, $\bar{P}_{h}: \Lambda^{n-2}(\Omega ; \mathbb{V}) \rightarrow \mathcal{P}_{r+2}^{-} \Lambda^{n-2}\left(\mathcal{T}_{h} ; \mathbb{V}\right)$. Namely, as for the canonical projection, $\bar{P}_{h} \omega$ is defined in terms of the degrees of freedom in (5.2), but it is taken to be the element of $\mathcal{P}_{r+2}^{-} \Lambda^{n-2}\left(\mathcal{T}_{h} ; \mathbb{V}\right)$ with the same moments as $\omega$ on the faces of codimension 0 and 1 , but with the moments of a smoothed approximation of $\omega$ on the faces of codimension 2. For more details see Arnold 
et al. $(2006 c)$. The properties we will need of this operator as well as the relevant canonical projections are summarized in the following lemma.

Lemma 11.3. Let

$$
\begin{aligned}
\Pi_{h}^{n-1}: \Lambda^{n-1}(\Omega ; \mathbb{K}) & \rightarrow \mathcal{P}_{r+1}^{-} \Lambda^{n-1}\left(\mathcal{T}_{h} ; \mathbb{K}\right) \\
\Pi_{h}^{n}: \Lambda^{n}(\Omega ; \mathbb{K}) & \rightarrow \mathcal{P}_{r} \Lambda^{n}\left(\mathcal{T}_{h} ; \mathbb{K}\right) \\
\bar{\Pi}_{h}^{n-1}: \Lambda^{n-1}(\Omega ; \mathbb{V}) & \rightarrow \mathcal{P}_{r+1} \Lambda^{n-1}\left(\mathcal{T}_{h} ; \mathbb{V}\right) \\
\bar{\Pi}_{h}^{n}: \Lambda^{n}(\Omega ; \mathbb{V}) & \rightarrow \mathcal{P}_{r} \Lambda^{n}\left(\mathcal{T}_{h} ; \mathbb{V}\right)
\end{aligned}
$$

be the canonical projections, and let $\bar{P}_{h}: \Lambda^{n-2}(\Omega ; \mathbb{V}) \rightarrow \mathcal{P}_{r+2}^{-} \Lambda^{n-2}\left(\mathcal{T}_{h} ; \mathbb{V}\right)$ be the operator described above. Then

$$
\begin{array}{cc}
\mathrm{d} \Pi_{h}^{n-1}=\Pi_{h}^{n} \mathrm{~d}, & \mathrm{~d} \bar{\Pi}_{h}^{n-1}=\bar{\Pi}_{h}^{n} \mathrm{~d}, \\
\Pi_{h}^{n-1} S_{n-2} \bar{P}_{h}=\Pi_{h}^{n-1} S_{n-2}, & \\
\left\|\Pi_{h}^{n} \omega\right\| \leq c\|\omega\|, & \omega \in \Lambda^{n}(\Omega ; \mathbb{K}), \\
\left\|\bar{\Pi}_{h}^{n-1} \omega\right\| \leq c\|\omega\|_{1}, & \\
\left\|\mathrm{~d} \bar{P}_{h} \eta\right\| \leq c\|\eta\|_{1} & \eta \in \Lambda^{n-1}(\Omega ; \mathbb{V}),
\end{array}
$$

The constant $c$ is uniform in the mesh size $h$ (although it may depend on the shape regularity of the mesh).

Proof. The commutativity conditions in (11.25) are the standard ones. We proved (11.26) with $\bar{\Pi}_{h}^{n-2}$ in place of $\bar{P}_{h}$ in Theorem 11.2. Since the proof only depended on the fact that the projection preserved the appropriate moments on faces of codimension 0 or 1 , the same proof works for $\bar{\Pi}_{h}^{n-2}$. The $L^{2}$ bound (11.27) is obvious since $\Pi_{h}^{n}$ is just the $L^{2}$-projection. The bound (11.28) is standard. Finally the bound in (11.29) can be proved using standard techniques; see Arnold et al. (2006c).

We can now state the main stability result, following the outline of Theorem 11.1.

Theorem 11.4. Given $(\omega, \mu) \in \mathcal{P}_{r} \Lambda^{n}\left(\mathcal{T}_{h} ; \mathbb{K}\right) \times \mathcal{P}_{r} \Lambda^{n}\left(\mathcal{T}_{h} ; \mathbb{V}\right)$, there exists $\sigma \in \mathcal{P}_{r+1} \Lambda^{n-1}\left(\mathcal{T}_{h} ; \mathbb{V}\right)$ such that $\mathrm{d} \sigma=\mu,-\Pi_{h}^{n} S_{n-1} \sigma=\omega$, and

$$
\|\sigma\|_{H \Lambda} \leq c(\|\omega\|+\|\mu\|)
$$

where the constant $c$ is independent of $\omega, \mu$ and $h$.

\section{Proof.}

(1) By Theorem 2.4, we can find $\eta \in H^{1} \Lambda^{n-1}(\Omega ; \mathbb{V})$ with $\mathrm{d} \eta=\mu$ and $\|\eta\|_{1} \leq c\|\mu\|$.

(2) Since $\omega+\Pi_{h}^{n} S_{n-1} \bar{\Pi}_{h}^{n-1} \eta \in H \Lambda^{n}(\Omega ; K)$, we can apply Theorem 2.4 a 
second time to find $\tau \in H^{1} \Lambda^{n-1}(\Omega ; \mathbb{K})$ with $\mathrm{d} \tau=\omega+\Pi_{h}^{n} S_{n-1} \bar{\Pi}_{h}^{n-1} \eta$ and $\|\tau\|_{1} \leq c\left(\|\omega\|+\left\|\Pi_{h}^{n} S_{n-1} \bar{\Pi}_{h}^{n-1} \eta\right\|\right)$.

(3) Since $S_{n-2}$ is an isomorphism from $H^{1} \Lambda^{n-2}(\Omega ; \mathbb{V})$ to $H^{1} \Lambda^{n-1}(\Omega ; \mathbb{K})$, we have $\rho \in H^{1} \Lambda^{n-2}(\Omega ; \mathbb{V})$ with $S_{n-2} \rho=\tau$, and $\|\rho\|_{1} \leq c\|\tau\|_{1}$.

(4) Define $\sigma=\mathrm{d} \bar{P}_{h} \rho+\bar{\Pi}_{h}^{n-1} \eta \in \mathcal{P}_{r+1} \Lambda^{n-1}\left(\mathcal{T}_{h} ; \mathbb{V}\right)$.

(5) From step (4), (11.25), step (1), and the fact that $\bar{\Pi}_{h}^{n}$ is a projection, we have

$$
\mathrm{d} \sigma=\mathrm{d} \bar{\Pi}_{h}^{n-1} \eta=\bar{\Pi}_{h}^{n} \mathrm{~d} \eta=\Pi_{h}^{n} \mu=\mu .
$$

(6) From step (4),

$$
-\Pi_{h}^{n} S_{n-1} \sigma=-\Pi_{h}^{n} S_{n-1} \mathrm{~d} \bar{P}_{h} \rho-\Pi_{h}^{n} S_{n-1} \bar{\Pi}_{h}^{n-1} \eta .
$$

Applying, in order, (11.7), (11.25), (11.26), step (3), (11.25), step (2), and the fact that $\Pi_{h}^{n}$ is a projection, we obtain

$$
\begin{aligned}
\Pi_{h}^{n} S_{n-1} \mathrm{~d} \bar{P}_{h} \rho & =-\Pi_{h}^{n} \mathrm{~d} S_{n-2} \bar{P}_{h} \rho=-\mathrm{d} \Pi_{h}^{n-1} S_{n-2} \bar{P}_{h} \rho \\
& =-\mathrm{d} \Pi_{h}^{n-1} S_{n-2} \rho=-\mathrm{d} \Pi_{h}^{n-1} \tau=-\Pi_{h}^{n} \mathrm{~d} \tau \\
& =-\Pi_{h}^{n}\left(\omega+\Pi_{h}^{n} S_{n-1} \bar{\Pi}_{h}^{n-1} \eta\right)=-\omega-\Pi_{h}^{n} S_{n-1} \bar{\Pi}_{h}^{n-1} \eta .
\end{aligned}
$$

Combining, we have $-\Pi_{h}^{n} S_{n-1} \sigma=\omega$.

(7) Finally, we prove the norm bound. From (11.27), the boundedness of $S_{n-1}$ in $L^{2},(11.28)$, and step (1),

$$
\left\|\Pi_{h}^{n} S_{n-1} \bar{\Pi}_{h}^{n-1} \eta\right\| \leq c\left\|S_{n-1} \bar{\Pi}_{h}^{n-1} \eta\right\| \leq c\left\|\bar{\Pi}_{h}^{n-1} \eta\right\| \leq c\|\eta\|_{1} \leq c\|\mu\| .
$$

Combining with the bounds in step (3) and (2), this gives $\|\rho\|_{1} \leq$ $c(\|\omega\|+\|\mu\|)$. From (11.29), we then have $\left\|\mathrm{d} \bar{P}_{h} \rho\right\| \leq c(\|\omega\|+\|\mu\|)$. From (11.28) and the bound in step (1), $\left\|\bar{\Pi}_{h}^{n-1} \eta\right\| \leq c\|\eta\|_{1} \leq c\|\mu\|$. In view of the definition of $\sigma$, these two last bounds imply that $\|\sigma\| \leq$ $c(\|\omega\|+\|\mu\|)$, while $\|\mathrm{d} \sigma\|=\|\mu\|$, and thus we have the desired bound $(11.30)$.

We have thus verified the stability conditions (S1) and (S2), and so may apply the standard theory of mixed methods (see Brezzi (1974), Brezzi and Fortin (1991), Douglas and Roberts (1985), Falk and Osborn (1980)) and standard results about approximation by finite element spaces to obtain convergence and error estimates.

Theorem 11.5. Suppose $(\sigma, u, p)$ is the solution of the elasticity system (11.4) and $\left(\sigma_{h}, u_{h}, p_{h}\right)$ is the solution of discrete system (11.5), where the 
finite element spaces are given by (11.24) for some integer $r \geq 0$. Then there is a constant $C$, independent of $h$, such that

$$
\begin{aligned}
\left\|\sigma-\sigma_{h}\right\|_{H \Lambda}+\left\|u-u_{h}\right\|+\| p & -p_{h} \| \\
& \leq C \inf \left(\|\sigma-\tau\|_{H \Lambda}+\|u-v\|+\|p-q\|\right) .
\end{aligned}
$$

where the infimum is over all $\tau \in \Lambda_{h}^{n-1}(\mathbb{V}), v \in \Lambda_{h}^{n}(\mathbb{V})$, and $q \in \Lambda_{h}^{n}(\mathbb{K})$. If $u$ and $\sigma$ are sufficiently smooth, then

$$
\begin{aligned}
\left\|\sigma-\sigma_{h}\right\|+\left\|u-u_{h}\right\|+\left\|p-p_{h}\right\| & \leq C h^{r+1}\|u\|_{r+2}, \\
\left\|\mathrm{~d}\left(\sigma-\sigma_{h}\right)\right\| & \leq C h^{r+1}\|\mathrm{~d} \sigma\|_{r+1} .
\end{aligned}
$$

\subsection{Traction boundary conditions}

So far we have considered only the case of the Dirichlet boundary condition $u=0$ on $\partial \Omega$. In this subsection, we consider the modifications that need to be made to deal with the case of the traction boundary condition $\sigma n=0$ on $\partial \Omega$. For this boundary value problem, in order for a solution to exist, $f$ must be orthogonal in $L^{2}(\Omega ; \mathbb{V})$ to the space $\mathbb{T}$ of rigid motions, defined to be the restrictions to $\Omega$ of affine maps of the form $x \mapsto a+b x$ where $a \in \mathbb{V}$ and $b \in \mathbb{K}$. If $f$ does satisfy this compatibility condition, then $u$ is only unique up to addition of a rigid motion. One method of defining a well-posed weak formulations for this problem is to introduce a Lagrange multiplier to enforce the constraint on $f$. We are then led to the following weak formulation, analogous to (11.3).

Find $(\sigma, u, p, s) \in \stackrel{\circ}{H}(\operatorname{div}, \Omega ; \mathbb{M}) \times L^{2}(\Omega ; \mathbb{V}) \times L^{2}(\Omega ; \mathbb{K}) \times \mathbb{T}$ satisfying

$$
\begin{aligned}
\int_{\Omega}(A \sigma: \tau+\operatorname{div} \tau \cdot u+\tau: p) \mathrm{d} x & =0, & & \tau \in \stackrel{\circ}{H}(\operatorname{div}, \Omega ; \mathbb{M}), \\
\int_{\Omega}(\operatorname{div} \sigma \cdot v+s \cdot v) \mathrm{d} x & =\int_{\Omega} f \cdot v \mathrm{~d} x, & & v \in L^{2}(\Omega ; \mathbb{V}), \\
\int_{\Omega} \sigma: q \mathrm{~d} x & =0, & & q \in L^{2}(\Omega ; \mathbb{K}), \\
\int_{\Omega} u \cdot t \mathrm{~d} x & =0, & & t \in \mathbb{T},
\end{aligned}
$$

where

$$
\stackrel{\circ}{H}(\operatorname{div}, \Omega ; \mathbb{M})=\{\sigma \in H(\operatorname{div}, \Omega ; \mathbb{M}): \sigma n=0 \text { on } \partial \Omega\} .
$$

We shall show below that this problem is well-posed.

To restate this in the language of differential forms we introduce $\mathbb{T}^{\star}=$ $\star \mathbb{T} \subset \mathcal{P}_{1} \Lambda^{n}(\Omega ; \mathbb{V})$. The problem then takes the form: given $f \in L^{2} \Lambda^{n}(\Omega ; \mathbb{V})$, 
find $(\sigma, u, p, s) \in \stackrel{\circ}{H} \Lambda^{n-1}(\Omega ; \mathbb{V}) \times L^{2} \Lambda^{n}(\Omega ; \mathbb{V}) \times L^{2} \Lambda^{n}(\Omega ; \mathbb{K}) \times \mathbb{T}^{\star}$ such that

$$
\begin{aligned}
\langle A \sigma, \tau\rangle+\langle\mathrm{d} \tau, u\rangle-\left\langle S_{n-1} \tau, p\right\rangle & =0, & & \tau \in \stackrel{\circ}{H} \Lambda^{n-1}(\Omega ; \mathbb{V}), \\
\langle\mathrm{d} \sigma, v\rangle+\langle s, v\rangle & =\langle f, v\rangle, & & v \in L^{2} \Lambda^{n}(\Omega ; \mathbb{V}), \\
\left\langle S_{n-1} \sigma, q\right\rangle & =0, & & q \in L^{2} \Lambda^{n}(\Omega ; \mathbb{K}), \\
\langle u, t\rangle & =0, & & t \in \mathbb{T}^{\star} .
\end{aligned}
$$

We remark that taking $v \in \mathbb{T}^{\star}$ in the second equation and using the identity given in Lemma 11.8 below together with the third equation, implies that $s$ is the $L^{2}$-projection of $f$ into $\mathbb{T}^{\star}$.

We consider here the development of stable mixed finite elements for the linear elasticity problem with traction boundary conditions based on the variational formulation (11.31). To do so, we will follow the development for the Dirichlet problem. In particular, we will again use the link between stable mixed finite elements for elasticity and the existence of discrete versions of a corresponding elasticity complex and also the connection between the elasticity complex and the ordinary de Rham complex. Thus, the choice of stable finite element spaces for elasticity with traction boundary conditions will again have as its starting point discrete versions of an appropriate de Rham complex. Since the derivation is quite analogous to the case of Dirichlet boundary conditions, we will not provide all the details, but concentrate on the modifications that are needed. We will make use of finite element spaces of the form $\AA_{h}^{k}:=\Lambda_{h}^{k} \cap \stackrel{\circ}{H} \Lambda^{k}$ (where $\Lambda_{h}^{k}=\mathcal{P}_{r} \Lambda^{k}\left(\mathcal{T}_{h}\right)$ or $\left.\mathcal{P}_{r}^{-} \Lambda^{k}\left(\mathcal{T}_{h}\right)\right)$. The canonical projection operator $\Pi_{h}^{k}$ then maps $\stackrel{\circ}{H} \Lambda^{k}(\Omega)$ into $\AA_{h}^{k}$.

We begin with the BGG construction, parallel to Section 11.2. For the case of traction boundary conditions, the appropriate de Rham sequence is that with compact support, (2.13), and the corresponding $L^{2}$ complex (2.14). So our starting complex for the BGG construction is

$$
0 \rightarrow \AA^{0}(\mathbb{W}) \stackrel{\left(\begin{array}{ll}
\mathrm{d} & 0 \\
0 & \mathrm{~d}
\end{array}\right)}{\longrightarrow} \AA^{1}(\mathbb{W}) \stackrel{\left(\begin{array}{ll}
\mathrm{d} & 0 \\
0 & \mathrm{~d}
\end{array}\right)}{\longrightarrow} \cdots \stackrel{\left(\begin{array}{ll}
\mathrm{d} & 0 \\
0 & \mathrm{~d}
\end{array}\right)}{\longrightarrow} \AA^{n}(\mathbb{W}) \rightarrow 0
$$

where $\mathbb{W}=\mathbb{K} \times \mathbb{V}$ and $\AA^{k}(\mathbb{W}):=\AA(\Omega ; \mathbb{K}) \times \AA(\Omega ; \mathbb{V})$. With $\Phi$ and $\mathcal{A}$ as before, $\Phi$ is a cochain isomorphism from (11.32) to

$$
0 \rightarrow \AA^{0}(\mathbb{W}) \stackrel{\mathcal{A}}{\rightarrow} \AA^{1}(\mathbb{W}) \stackrel{\mathcal{A}}{\rightarrow} \cdots \stackrel{\mathcal{A}}{\rightarrow} \AA^{n}(\mathbb{W}) \rightarrow 0 .
$$

Introducing the spaces $\stackrel{\circ}{\Gamma}^{i}$ in analogy to the spaces $\Gamma^{i}$ of Section 11.2, we obtain the subcomplex

$$
0 \rightarrow \AA^{0}(\mathbb{W}) \stackrel{\mathcal{A}}{\longrightarrow} \cdots \stackrel{\mathcal{A}}{\longrightarrow} \AA^{n-3}(\mathbb{W}) \stackrel{\mathcal{A}}{\rightarrow} \stackrel{\circ}{\Gamma}^{n-2} \stackrel{\mathcal{A}}{\longrightarrow} \stackrel{\circ}{\Gamma}^{n-1} \stackrel{\mathcal{A}}{\longrightarrow} \AA^{n}(\mathbb{W}) \rightarrow 0,
$$

and a corresponding cochain projection. Identifying elements $(\omega, \mu) \in \stackrel{\circ}{\Gamma}^{n-2}$ 
with $\omega \in \stackrel{\circ}{\Lambda}^{n-2}(\Omega ; \mathbb{K})$ and elements $(0, \mu) \in \stackrel{\circ}{\Gamma}^{n-1}$ with $\mu \in \stackrel{\circ}{\Lambda}^{n-1}(\Omega ; \mathbb{V})$, we obtain the relevant elasticity complex

$$
\begin{aligned}
0 \rightarrow \AA^{0}(\mathbb{W}) \stackrel{\mathcal{A}}{\longrightarrow} & \stackrel{\mathcal{A}}{\longrightarrow} \AA^{n-3}(\mathbb{W}) \stackrel{\left(\mathrm{d},-S_{n-3}\right)}{\longrightarrow} \AA^{n-2}(\Omega ; \mathbb{K}) \\
& \stackrel{\mathrm{do} S_{n-2}^{-1} \text { od }}{\longrightarrow} \AA^{n-1}(\Omega ; \mathbb{V}) \stackrel{\left(-S_{n-1}, \mathrm{~d}\right)^{T}}{\longrightarrow} \AA^{n}(\mathbb{W}) \rightarrow 0 .
\end{aligned}
$$

The complex (11.32), and so the isomorphic complex (11.33) have a cohomology space of dimension $\operatorname{dim} \mathbb{V}+\operatorname{dim} \mathbb{K}=n(n+1) / 2$ at the highest order. Thus the highest-order cohomology space for the elasticity complex (11.34) has dimension at most $n(n+1) / 2$. In other words, solvability of the problem, given $(\omega, \mu) \in \AA^{n}(\mathbb{W})$, to find $\sigma \in \AA^{n-1}(\Omega ; \mathbb{V})$ such that

$$
\left(-S_{n-1} \sigma, \mathrm{d} \sigma\right)=(\omega, \mu)
$$

implies at most $n(n+1) / 2$ constraints on the data $(\omega, \mu)$. In fact, it implies exactly this many constraints, namely,

$$
\int_{\Omega} \mu=0, \quad \int_{\Omega} \omega=\int_{\Omega} K \mu \text {. }
$$

Indeed, the first equation ( $n$ constraints) follows immediately from the equation $\mathrm{d} \sigma=\mu$ and Stokes' theorem, while

$$
\int_{\Omega} \omega=\int_{\Omega}(K d-d K) \sigma=\int_{\Omega} K \mathrm{~d} \sigma=\int_{\Omega} \mu .
$$

Our next goal is to prove the well-posedness of (11.31). But first we prove a useful lemma.

Lemma 11.6. Given $a \in \mathbb{V}$ and $b \in \mathbb{K}$, there exists a unique $s \in \mathbb{T}^{\star}$ such that $\int_{\Omega} s=a, \int_{\Omega} K s=b$.

Proof. Since $\operatorname{dim} \mathbb{T}^{\star}=\operatorname{dim} \mathbb{V}+\operatorname{dim} \mathbb{K}$, it is enough to show that if $\int_{\Omega} s$ and $\int_{\Omega} K s=0$, then $s=0$. Now $s(x)=(g+c x)$ vol for some $g \in \mathbb{V}$ and $c \in \mathbb{K}$. From the vanishing of $\int s$ we can write $g$ in terms of $c$ and find that $s(x)=c(x-\bar{x})$ vol where $\bar{x}$ is the barycentre of $\Omega$. We have a simple linear algebra identity

$$
\langle v \wedge b v, b\rangle=2|b v|^{2}, \quad v \in \mathbb{V}, b \in \mathbb{K},
$$

where the inner product on the left is taken in $\mathbb{M}$ and the norm on the right is the norm in $\mathbb{V}$. Thus

$$
\left.|s(x)|^{2}=|c(x-\bar{x})|^{2}=\frac{1}{2}\langle(x-\bar{x}) \wedge c(x-\bar{x}), c\rangle . s(x), c\right\rangle .
$$

Integrating over $\Omega$ and using the fact that $\int s=0$, we get

$$
\int_{\Omega}|s(x)|^{2} \mathrm{vol}=\frac{1}{2}\left\langle\int_{\Omega} x \wedge s(x), c\right\rangle=\frac{1}{2}\left\langle\int_{\Omega} K s, c\right\rangle=0 .
$$


We now turn to the proof of well-posedness, i.e., the analogue of Theorem 11.1 for traction boundary conditions. The problem (11.31) is of the saddle point type to which Brezzi's theorem applies, with

$$
\begin{aligned}
& a(\sigma, s ; \tau, t)=\langle A \sigma, \tau\rangle, \\
& b(\sigma, s ; v, q)=\langle\mathrm{d} \sigma, v\rangle-\left\langle S_{n-1} \sigma, q\right\rangle+\langle s, v\rangle .
\end{aligned}
$$

The analogues of conditions (W1) and (W2) are:

$\left(\mathrm{W} 1^{\prime}\right)\|\tau\|_{H \Lambda}^{2}+\|t\|^{2} \leq c_{1}\langle A \tau, \tau\rangle$ whenever $\tau \in \stackrel{\circ}{H} \Lambda^{n-1}(\Omega ; \mathbb{V})$ and $t \in \mathbb{T}^{\star}$ satisfy $\langle\mathrm{d} \tau, v\rangle+\langle t, v\rangle=0 \quad \forall v \in L^{2} \Lambda^{n}(\Omega ; \mathbb{V})$ and $\left\langle S_{n-1} \tau, q\right\rangle=0$ $\forall q \in L^{2} \Lambda^{n}(\Omega ; \mathbb{K})$,

$\left(\mathrm{W} 2^{\prime}\right)$ for all nonzero $(v, q) \in \Lambda^{n}(\Omega ; \mathbb{V}) \times \Lambda^{n}(\Omega ; \mathbb{K})$, there exists nonzero $\tau \in \AA^{n-1}(\Omega ; \mathbb{V})$ and $t \in \mathbb{T}^{\star}$ with

$$
\langle\mathrm{d} \tau, v\rangle-\left\langle S_{n-1} \tau, q\right\rangle+\langle t, v\rangle \geq c_{2}\left(\|\tau\|_{H \Lambda}+\|t\|\right)(\|v\|+\|q\|),
$$

for some positive constants $c_{1}$ and $c_{2}$. Again, the first condition is easy (since for such $\tau$ and $t$, the rigid motion $t$ is an $L^{2}$-projection of $\mathrm{d} \tau$ ). We now prove $\left(\mathrm{W} 2^{\prime}\right)$.

Theorem 11.7. Given $(\omega, \mu) \in L^{2} \Lambda^{n}(\Omega ; \mathbb{K}) \times L^{2} \Lambda^{n}(\Omega ; \mathbb{V})$, there exists $\sigma \in H \Lambda^{n-1}(\Omega ; \mathbb{V}), s \in \mathbb{T}^{\star}$ such that $\mathrm{d} \sigma+s=\mu,-S_{n-1} \sigma=\omega$. Moreover we may choose $\sigma, s$ so that

$$
\|\sigma\|_{H \Lambda}+\|s\| \leq c(\|\omega\|+\|\mu\|),
$$

for a fixed constant $c$.

Proof. Again, the norm bound is automatic once the existence is established.

(0) Define $s \in \mathbb{T}^{*}$ by

$$
\int_{\Omega} s=\int_{\Omega} \mu, \quad \int_{\Omega} K s=\int_{\Omega}(K \mu+\omega) .
$$

By the lemma, this determines $s$.

(1) By Theorem 2.4, we can find $\eta \in \stackrel{\circ}{H}^{1} \Lambda^{n-1}(\Omega ; \mathbb{V})$ with $\mathrm{d} \eta=\mu-s$.

(2) Now $\omega+S_{n-1} \eta \in H \Lambda^{n}(\Omega ; K)$ and has vanishing integral, since

$$
\int_{\Omega} S_{n-1} \eta=\int_{\Omega} K \mathrm{~d} \eta=\int_{\Omega} K(\mu-s)=-\int_{\Omega} \omega .
$$

Thus we can apply Theorem 2.4 another time to find $\tau \in \stackrel{\circ}{H}^{1} \Lambda^{n-1}(\Omega ; \mathbb{K})$ with $\mathrm{d} \tau=\omega+S_{n-1} \eta$.

(3) Take $\rho \in \stackrel{\circ}{H}^{1} \Lambda^{n-2}(\Omega ; \mathbb{V})$ with $S_{n-2} \rho=\tau$. 
(4) Define $\sigma=\mathrm{d} \rho+\eta \in \stackrel{\circ}{H} \Lambda^{n-1}(\Omega ; \mathbb{V})$.

(5) From steps (1) and (4), it is immediate that $\mathrm{d} \sigma+s=\mu$.

(6) From (4),

$$
-S_{n-1} \sigma=-S_{n-1} \mathrm{~d} \rho-S_{n-1} \eta=d S_{n-2} \rho-S_{n-1} \eta=\mathrm{d} \tau-S_{n-1} \eta=\omega .
$$

We now turn to the discrete problem: find $\left(\sigma_{h}, u_{h}, p_{h}, s_{h}\right) \in \AA_{h}^{n-1}(\mathbb{V}) \times$ $\Lambda_{h}^{n}(\mathbb{V}) \times \Lambda_{h}^{n}(\mathbb{K}) \times \mathbb{T}^{\star}$ such that

$$
\begin{aligned}
\left\langle A \sigma_{h}, \tau\right\rangle+\left\langle\mathrm{d} \tau, u_{h}\right\rangle-\left\langle S_{n-1} \tau, p_{h}\right\rangle & =0, & & \tau \in \AA_{h}^{n-1}(\mathbb{V}), \\
\left\langle\mathrm{d} \sigma_{h}, v\right\rangle+\left\langle s_{h}, v\right\rangle & =\langle f, v\rangle, & & v \in \Lambda_{h}^{n}(\mathbb{V}), \\
\left\langle S_{n-1} \sigma_{h}, q\right\rangle & =0, & & q \in \Lambda_{h}^{n}(\mathbb{K}), \\
\left\langle u_{h}, t\right\rangle & =0, & & t \in \mathbb{T}^{\star} .
\end{aligned}
$$

The stability conditions for this system are then:

$\left(\mathrm{S}^{\prime}\right)\|\tau\|_{H \Lambda}^{2}+\|s\|^{2} \leq c_{1}\langle A \tau, \tau\rangle$ whenever $\tau \in \AA_{h}^{n-1}(\mathbb{V})$ and $s \in \mathbb{T}^{\star}$ satisfy $\langle\mathrm{d} \tau, v\rangle+\langle s, v\rangle=0 \quad \forall v \in \Lambda_{h}^{n}(\mathbb{V})$ and $\left\langle S_{n-1} \tau, q\right\rangle=0 \forall q \in \Lambda_{h}^{n}(\mathbb{K})$,

$\left(\mathrm{S} 2^{\prime}\right)$ for all nonzero $(v, q) \in \Lambda_{h}^{n}(\mathbb{V}) \times \Lambda_{h}^{n}(\mathbb{K})$, there exists nonzero $\tau \in$ $\bigwedge_{h}^{n-1}(\mathbb{V})$ and $s \in \mathbb{T}^{\star}$ with

$$
\langle\mathrm{d} \tau, v\rangle-\left\langle S_{n-1} \tau, q\right\rangle+\langle s, v\rangle \geq c_{2}\left(\|\tau\|_{H \Lambda}+\|s\|\right)(\|v\|+\|q\|),
$$

where $c_{1}$ and $c_{2}$ are positive constants independent of $h$.

We choose the same finite element spaces as before, except that the stress space now incorporates the boundary conditions:

$$
\begin{gathered}
\stackrel{\circ}{h}_{h}^{n-1}(\mathbb{V})=\stackrel{\circ}{\mathcal{P}}_{r+1} \Lambda^{n-1}\left(\mathcal{T}_{h} ; \mathbb{V}\right), \quad \Lambda_{h}^{n}(\mathbb{V})=\mathcal{P}_{r} \Lambda^{n}\left(\mathcal{T}_{h} ; \mathbb{V}\right), \\
\Lambda_{h}^{n}(\mathbb{K})=\mathcal{P}_{r} \Lambda^{n}\left(\mathcal{T}_{h} ; \mathbb{K}\right) .
\end{gathered}
$$

We show that, for any $r \geq 0$, this choice gives a stable finite element discretization of the system (11.36). The case when $r=0$ requires a bit of extra effort, because $\mathbb{T}^{\star} \nsubseteq \Lambda_{h}^{n}(\mathbb{V})$ in this case. We begin by assuming that $r \geq 1$ and remark on the case $r=0$ at the end.

The following simple identity will be useful in establishing stability.

Lemma 11.8. Let $s=(a+b x)$ vol $\in \mathbb{T}^{\star}$, with $a \in \mathbb{V}$ and $b \in \mathbb{K}$. Then

$$
\langle\mathrm{d} \tau, s\rangle=-\left\langle S_{n-1} \tau, b \text { vol }\right\rangle, \quad \sigma \in \stackrel{\circ}{H} \Lambda^{n-1}(\Omega ; \mathbb{V}) .
$$

First we verify the stability $\left(\mathrm{S}^{\prime}\right)$. We have $\tau \in \stackrel{\circ}{\mathcal{P}}_{r+1} \Lambda^{n-1}\left(\mathcal{T}_{h} ; \mathbb{V}\right)$ and $s \in \mathbb{T}^{\star}$ with

$$
\langle\mathrm{d} \tau, v\rangle+\langle s, v\rangle=0, v \in \mathcal{P}_{r} \Lambda^{n}\left(\mathcal{T}_{h} ; \mathbb{V}\right), \quad\left\langle S_{n-1} \tau, q\right\rangle=0, q \in \mathcal{P}_{r} \Lambda^{n}\left(\mathcal{T}_{h} ; \mathbb{K}\right) .
$$

Taking $v=s$ in the first equation, applying the lemma, and then the second 
equation, we conclude that $s=0$. Then we take $v=\mathrm{d} \tau$ and conclude that $\mathrm{d} \tau=0$, so the bound in $\left(\mathrm{S}^{\prime}\right)$ holds.

The proof of the second stability condition is very much as in the case of Dirichlet boundary conditions, with the minor extra complications which we have already seen in the continuous case in Theorem 11.7, so we just sketch the proof.

Theorem 11.9. Given $(\omega, \mu) \in \mathcal{P}_{r} \Lambda^{n}\left(\mathcal{T}_{h} ; \mathbb{K}\right) \times \mathcal{P}_{r} \Lambda^{n}\left(\mathcal{T}_{h} ; \mathbb{V}\right)$, there exists $\sigma \in \stackrel{\circ}{\mathcal{P}}_{r+1} \Lambda^{n-1}\left(\mathcal{T}_{h} ; \mathbb{V}\right)$ and $s \in \mathbb{T}^{\star}$ such that

$$
\mathrm{d} \sigma+s=\mu, \quad-\Pi_{h}^{n} S_{n-1} \sigma=\omega,
$$

and

$$
\|\sigma\|_{H \Lambda}+\|s\| \leq c(\|\omega\|+\|\mu\|),
$$

where the constant $c$ is independent of $\omega, \mu$ and $h$.

Proof. First define $s$ and $\eta$ as in steps (0) and (1) of Theorem 11.7. Note that

$$
\begin{aligned}
\int_{\Omega}\left(\omega+\Pi_{h}^{n} S_{n-1} \bar{\Pi}_{h}^{n-1} \eta\right) & =\int_{\Omega}\left(\omega-K \mathrm{~d} \bar{\Pi}_{h}^{n-1} \eta\right)=\int_{\Omega}\left(\omega-K \bar{\Pi}_{h}^{n}(\mu-s)\right) \\
& =\int_{\Omega}(\omega-K(\mu-s))=0 .
\end{aligned}
$$

Thus we can take $\tau \in \stackrel{\circ}{H} \Lambda^{n-1}(\Omega ; \mathbb{K})$ with $\mathrm{d} \tau=\omega+\Pi_{h}^{n} S_{n-1} \bar{\Pi}_{h}^{n-1} \eta$ and then $\rho=S_{n-2}^{-1} \tau$, and $\sigma=\mathrm{d} \bar{P}_{h}+\bar{\Pi}_{h}^{n-1} \eta$. The remainder of the proof is just as for Theorem 11.4.

Finally we remark on the modifications that have to be made in the case $r=0$. In the proof of $\left(\mathrm{S}^{\prime}\right)$, we cannot take $v=s$, since $\mathbb{T}^{\star} \nsubseteq \mathcal{P}_{0} \Lambda^{n}\left(\mathcal{T}_{h} ; \mathbb{V}\right)$. So we take $v=\bar{\Pi}_{h}^{n} s$, with $\bar{\Pi}_{h}^{n}$ simply the $L^{2}$-projection into the piecewise constant $n$-forms. Then

$$
\left\|\bar{\Pi}_{h}^{n} s\right\|^{2}=\langle s, v\rangle=-\langle\mathrm{d} \tau, v\rangle=-\langle\mathrm{d} \tau, s\rangle=0,
$$

with the last step following from the lemma, as before. The $\bar{\Pi}_{h}^{n} s=0$. But for $s \in \mathbb{T}^{\star}$ it is easy to see that this implies that $s=0$, at least for $h$ sufficiently small.

A similar issue arises in the verification of $\left(\mathrm{S}^{\prime}\right)$. Now we want to find $\sigma \in \stackrel{\circ}{\mathcal{P}}_{1} \Lambda^{n-1}\left(\mathcal{T}_{h} ; \mathbb{V}\right), s \in \mathbb{T}^{\star}$ with $\mathrm{d} \sigma+\bar{\Pi}_{h}^{n} s=\mu$. This requires us to define $s$ by

$$
\int_{\Omega} s=\int_{\Omega} \mu, \quad \int_{\Omega} K \bar{\Pi}_{h}^{n} s=\int_{\Omega}(K \mu+\omega) .
$$

The existence of such an $s$ follows from a variant of Lemma 11.6 which replaces $K s$ by $K \bar{\Pi}_{h}^{n} s$. The variant lemma can be proved using the identity (11.35), but taking $v=\bar{\Pi}_{h}(x-\bar{x})$, the $L^{2}$-projection of $x-\bar{x}$ into the 
piecewise constants, rather than $v=x-\bar{x}$ as before. It follows that $\bar{\Pi}_{h}^{n} s=0$, which again implies that $s$ vanishes for small $h$.

\subsection{Simplified elements}

The purpose of this subsection is to present a stable element for which the finite element spaces are slightly smaller than the simplest element derived so far, namely

$$
\Lambda_{h}^{n-1}(\mathbb{V})=\mathcal{P}_{1} \Lambda^{n-1}\left(\mathcal{T}_{h} ; \mathbb{V}\right), \Lambda_{h}^{n}(\mathbb{V})=P_{0} \Lambda^{n}\left(\mathcal{T}_{h} ; \mathbb{V}\right), \Lambda_{h}^{n}(\mathbb{K})=\mathcal{P}_{0} \Lambda^{n}\left(\mathcal{T}_{h} ; \mathbb{K}\right)
$$

(We return to the Dirichlet problem for this.) In the new element, the spaces $\Lambda_{h}^{n}(\mathbb{V})$ and $\Lambda_{h}^{n}(\mathbb{K})$ are unchanged, but $\Lambda_{h}^{n-1}(\mathbb{V})$ will be reduced from a full space of piecewise linear elements to one where some of the components are only a reduced space of linears. Since the full details for the cases $n=2$ and $n=3$ are provided in Arnold et al. (2006c) and Arnold et al. (2005), we only present the main ideas here.

By examining the proof of Theorem 11.4, we realize that we do not use the complete sequence (11.17) for the given spaces. We only use the sequences

$$
\begin{aligned}
& \mathcal{P}_{1}^{-} \Lambda^{n-1}\left(\mathcal{T}_{h} ; \mathbb{K}\right) \stackrel{\mathrm{d}}{\longrightarrow} \mathcal{P}_{0} \Lambda^{n}\left(\mathcal{T}_{h} ; \mathbb{K}\right) \longrightarrow 0, \\
& \mathcal{P}_{2}^{-} \Lambda^{n-2}\left(\mathcal{T}_{h} ; \mathbb{V}\right) \stackrel{\mathrm{d}}{\longrightarrow} \mathcal{P}_{1} \Lambda^{n-1}\left(\mathcal{T}_{h} ; \mathbb{V}\right) \stackrel{\mathrm{d}}{\longrightarrow} \mathcal{P}_{0} \Lambda^{n}\left(\mathcal{T}_{h} ; \mathbb{V}\right) \longrightarrow 0 .
\end{aligned}
$$

The purpose here is to show that it is possible to choose subspaces of some of the spaces in (11.37) such that the desired properties still hold. More precisely, compared to $(11.37)$, the spaces $\mathcal{P}_{2}^{-} \Lambda^{n-2}\left(\mathcal{T}_{h} ; \mathbb{V}\right)$ and $\mathcal{P}_{1} \Lambda^{n-1}\left(\mathcal{T}_{h} ; \mathbb{V}\right)$ are simplified, while the three others remain unchanged. If we denote by $\mathcal{P}_{2,-}^{-} \Lambda^{n-2}\left(\mathcal{T}_{h} ; \mathbb{V}\right)$ and $\mathcal{P}_{1,-} \Lambda^{n-1}\left(\mathcal{T}_{h} ; \mathbb{V}\right)$ the simplifications of the spaces $\mathcal{P}_{2}^{-} \Lambda^{n-2}\left(\mathcal{T}_{h} ; \mathbb{V}\right)$ and $\mathcal{P}_{1} \Lambda^{n-1}\left(\mathcal{T}_{h} ; \mathbb{V}\right)$, respectively, then the properties we need are that:

$$
\mathcal{P}_{2,-}^{-} \Lambda^{n-2}\left(\mathcal{T}_{h} ; \mathbb{V}\right) \stackrel{\mathrm{d}}{\longrightarrow} \mathcal{P}_{1,-} \Lambda^{n-1}\left(\mathcal{T}_{h} ; \mathbb{V}\right) \stackrel{\mathrm{d}}{\longrightarrow} \mathcal{P}_{0} \Lambda^{n}\left(\mathcal{T}_{h} ; \mathbb{V}\right) \longrightarrow 0
$$

is a complex and that the surjectivity assumption (11.20) holds, i.e., $S_{h}=$ $S_{n-2, h}$ maps $\mathcal{P}_{2,-}^{-} \Lambda^{n-2}\left(\mathcal{T}_{h} ; \mathbb{V}\right)$ onto $\mathcal{P}_{1}^{-} \Lambda^{n-1}\left(\mathcal{T}_{h} ; \mathbb{K}\right)$. We note that if the space $\mathcal{P}_{1}^{-} \Lambda^{n-1}\left(\mathcal{T}_{h} ; \mathbb{V}\right) \subset \mathcal{P}_{1,-} \Lambda^{n-1}\left(\mathcal{T}_{h} ; \mathbb{V}\right)$, then d maps $\mathcal{P}_{1,-} \Lambda^{n-1}\left(\mathcal{T}_{h} ; \mathbb{V}\right)$ onto $\mathcal{P}_{0} \Lambda^{n}\left(\mathcal{T}_{h} ; \mathbb{V}\right)$.

The key to this construction is to first show that a space $\mathcal{P}_{2,-}^{-} \Lambda^{n-2}\left(\mathcal{T}_{h} ; \mathbb{V}\right)$

can be constructed as a subspace of $\mathcal{P}_{2}^{-} \Lambda^{n-2}\left(\mathcal{T}_{h} ; \mathbb{V}\right)$, while still retaining the surjectivity assumption (11.20). This can be done locally on each simplex. We begin by recalling that the degrees of freedom on a face $f \in \Delta_{n-1}(T)$ of $\mathcal{P}_{2}^{-} \Lambda^{n-2}(T ; \mathbb{V})$ have the form

$$
\int_{f} \omega \wedge \mu, \quad \mu \in \mathcal{P}_{0} \Lambda^{1}(f, \mathbb{V})
$$


However, if we examine the proof of Theorem 11.2, we see that the only degrees of freedom that are used for an element $\omega \in \mathcal{P}_{2}^{-} \Lambda^{n-2}(T ; \mathbb{V})$ are the subset of the $\Delta_{n-1}(T)$ face degrees of freedom given by

$$
\int_{f} \omega \wedge \nu, \quad \nu \in \mathbb{K}
$$

where in the integral we view $\nu \in \mathbb{K} \subset \mathbb{M} \cong \operatorname{Alt}^{1}(\mathbb{V} ; \mathbb{V})$ as a 1 -form with values in $\mathbb{V}$.

To classify the degrees of freedom that we need to retain to establish Theorem 11.2 , we observe that the $n(n-1)$-dimensional space of test functions used in (11.39) can be decomposed into

$$
\mathcal{P}_{0} \Lambda^{1}(f ; \mathbb{V})=\mathcal{P}_{0} \Lambda^{1}\left(f ; T_{f}\right)+\mathcal{P}_{0} \Lambda^{1}\left(f ; N_{f}\right)
$$

i.e., into forms with values in the tangent space to $f, T_{f}$, or its orthogonal complement, $N_{f}$. This is an $(n-1)^{2}+(n-1)$-dimensional decomposition. Furthermore,

$$
\mathcal{P}_{0} \Lambda^{1}\left(f ; T_{f}\right)=\mathcal{P}_{0} \Lambda_{\text {sym }}^{1}\left(f ; T_{f}\right)+\mathcal{P}_{0} \Lambda_{\text {skw }}^{1}\left(f ; T_{f}\right),
$$

where $\mu \in \mathcal{P}_{0} \Lambda^{1}\left(f ; T_{f}\right)$ is in $\mathcal{P}_{0} \Lambda_{\text {sym }}^{1}\left(f ; T_{f}\right)$ if and only if $\mu(s) \cdot t=\mu(t) \cdot s$ for orthonormal tangent vectors $s$ and $t$. Note that when $n=2$, this space is 1-dimensional, so there is only $\mathcal{P}_{0} \Lambda_{\text {sym }}^{1}\left(f ; T_{f}\right)$. Finally, we obtain an $n(n-1) / 2+n(n-1) / 2$-dimensional decomposition

$$
\mathcal{P}_{0} \Lambda^{1}(f ; \mathbb{V})=\mathcal{P}_{0} \Lambda_{\text {sym }}^{1}\left(f ; T_{f}\right)+\mathcal{P}_{0} \Lambda_{\text {skw }}^{1}(f ; \mathbb{V}),
$$

where

$$
\mathcal{P}_{0} \Lambda_{\text {skw }}^{1}(f ; \mathbb{V})=\mathcal{P}_{0} \Lambda_{\text {skw }}^{1}\left(f ; T_{f}\right)+\mathcal{P}_{0} \Lambda^{1}\left(f ; N_{f}\right)
$$

It can be shown that the degrees of freedom corresponding to $\mathcal{P}_{0} \Lambda_{\text {skw }}^{1}(f ; \mathbb{V})$ are the ones that need to be retained, while those in $\mathcal{P}_{0} \Lambda_{\text {sym }}^{1}\left(f ; T_{f}\right)$ are not needed.

The reduced space $\mathcal{P}_{2,-}^{-} \Lambda^{n-2}(T ; \mathbb{V})$ that we now construct has two properties. The first is that it still contains the space $\mathcal{P}_{1} \Lambda^{n-2}(T ; \mathbb{V})$ and the second is that the unused face degrees of freedom are eliminated (by setting them equal to zero). We can achieve these conditions by first writing an element $\omega \in \mathcal{P}_{2}^{-} \Lambda^{n-2}(T ; \mathbb{V})$ as $\omega=\bar{\Pi}_{h} \omega+\left(I-\bar{\Pi}_{h}\right) \omega$, where $\bar{\Pi}_{h}$ denotes the usual projection operator into $\mathcal{P}_{1} \Lambda^{n-2}(T ; \mathbb{V})$ defined by the moments on the faces $f \in \Delta_{n-2}(T)$. Then the elements in $\left(I-\bar{\Pi}_{h}\right) \mathcal{P}_{2}^{-} \Lambda^{n-2}(T ; \mathbb{V})$ will have zero traces on these faces, so they are completely defined by their degrees of freedom on the faces $f \in \Delta_{n-1}(T)$ :

$$
\int_{f} \omega \wedge \mu, \quad \mu \in \mathcal{P}_{0} \Lambda^{1}(f ; \mathbb{V}), \quad f \in \Delta_{n-1}(T) .
$$

Thus, we henceforth denote $\left(I-\bar{\Pi}_{h}\right) \mathcal{P}_{2}^{-} \Lambda^{n-2}(T ; \mathbb{V})$ by $\mathcal{P}_{2, f}^{-} \Lambda^{n-2}(T ; \mathbb{V})$. 
We then define our reduced space

$$
\mathcal{P}_{2,-}^{-} \Lambda^{n-2}(T ; \mathbb{V})=\mathcal{P}_{1} \Lambda^{n-2}(T ; \mathbb{V})+\mathcal{P}_{2, f,-}^{-} \Lambda^{n-2}(T ; \mathbb{V})
$$

where $\mathcal{P}_{2, f,-}^{-} \Lambda^{n-2}(T ; \mathbb{V})$ denotes the set of forms $\omega \in \mathcal{P}_{2, f}^{-} \Lambda^{n-2}(T ; \mathbb{V})$ satisfying

$$
\int_{f} \omega \wedge \mu=0, \quad \mu \in \mathcal{P}_{0} \Lambda_{\mathrm{sym}}^{1}(f ; \mathbb{V}),
$$

i.e., we have set the unused degrees of freedom to be zero. The space $\mathcal{P}_{2,-}^{-} \Lambda_{h}^{2}(\mathbb{V})$ can then be defined from the local spaces in the usual way. The degrees of freedom for this space are then given by

$$
\begin{aligned}
& \int_{f} \omega \wedge \mu, \quad \mu \in \mathcal{P}_{1} \Lambda^{0}(f ; \mathbb{V}), \quad f \in \Delta_{n-2}(T), \\
& \int_{f} \omega \wedge \mu, \quad \mu \in \mathcal{P}_{0} \Lambda_{\text {skw }}^{1}(f ; \mathbb{V}), \quad f \in \Delta_{n-1}(T) .
\end{aligned}
$$

When $n=3$, the space $\mathcal{P}_{2,-}^{-} \Lambda^{1}(T ; \mathbb{V})$ will have 48 degrees of freedom $(36$ edge degrees of freedom and 12 face degrees of freedom).

The motivation for this choice of the space $\mathcal{P}_{2,-}^{-} \Lambda_{h}^{n-2}(\mathbb{V})$ is that it easily leads to a definition of the space $\mathcal{P}_{1,-} \Lambda_{h}^{n-1}(\mathbb{V})$ that satisfies the property that (11.38) is a complex. We begin by defining

$$
\mathcal{P}_{1,-} \Lambda^{n-1}(T ; \mathbb{V})=\mathcal{P}_{1}^{-} \Lambda^{n-1}(T ; \mathbb{V})+\mathrm{d} \mathcal{P}_{2, f,-}^{-} \Lambda^{n-2}(T ; \mathbb{V})
$$

When $n=3$, this space will have 24 face degrees of freedom. The space $\mathcal{P}_{1,-} \Lambda_{h}^{n-1}(\mathbb{V})$ is then defined from the local spaces in the usual way. It is clear that $\mathcal{P}_{1}^{-} \Lambda_{h}^{n-1}(\mathbb{V}) \subset \mathcal{P}_{1,-} \Lambda_{h}^{n-1}(\mathbb{V})$ and easy to check that the complex (11.38) is exact.

We define appropriate degrees of freedom for the space $\mathcal{P}_{1,-} \Lambda^{n-1}(T ; \mathbb{V})$ by using a subset of the degrees of freedom for $\mathcal{P}_{1} \Lambda^{n-1}(T ; \mathbb{V})$, i.e., of $\int_{f} \omega \wedge \mu$, $\mu \in \mathcal{P}_{1} \Lambda^{0}(f ; \mathbb{V}), f \in \Delta_{n-1}(T)$. In particular, we take as degrees of freedom for $\mathcal{P}_{1,-} \Lambda^{n-1}(T ; \mathbb{V})$,

$$
\int_{f} \omega \wedge \mu, \quad \mu \in \mathcal{P}_{1, \mathrm{skw}} \Lambda^{0}(f ; \mathbb{V}), \quad f \in \Delta_{n-1}(T),
$$

where $\mathcal{P}_{1, \mathrm{skw}} \Lambda^{0}(f ; \mathbb{V})$ denotes the set of $\mu \in \mathcal{P}_{1} \Lambda^{0}(f ; \mathbb{V})$ that satisfy $\mathrm{d} \mu \in$ $\mathcal{P}_{0} \Lambda_{\text {skw }}^{1}(f ; \mathbb{V})$.

Using an argument parallel to that used previously, it is straightforward to show that the simplified spaces also satisfy the surjectivity assumption (11.20). We can then complete the proof of stability and show that the convergence asserted in Theorem 11.5 for $r=0$ holds also for the reduced spaces.

When $n=3, \mathcal{P}_{1, \mathrm{skw}} \Lambda^{0}(f ; \mathbb{V})$ is a 6 -dimensional space on each face, so the 
above quantities specify 24 degrees of freedom for the space $\mathcal{P}_{1,-} \Lambda^{2}(T ; \mathbb{V})$. It is not difficult to check that these are a unisolvent set of degrees of freedom, and we can use the identification of an element $\omega \in \Lambda^{2}(\Omega ; \mathbb{V})$ with a matrix $F$ given by $\omega\left(v_{1}, v_{2}\right)=F\left(v_{1} \times v_{2}\right)$ to describe the six degrees of freedom on a face:

$$
\begin{aligned}
& \int_{f} F n \mathrm{~d} f \\
& \int_{f}(x \cdot t) n^{T} F n \mathrm{~d} f, \\
& \int_{f}(x \cdot s) n^{T} F n \mathrm{~d} f \\
& \int_{f}\left[(x \cdot t) s^{T}-(x \cdot s) t^{T}\right] F n \mathrm{~d} f .
\end{aligned}
$$

\section{Acknowledgments}

This work benefited substantially from the facilities and programming provided by the Institute for Mathematics and its Applications (IMA) at the University of Minnesota and by the Centre of Mathematics for Applications (CMA) at the University of Oslo. The workshop entitled Compatible Spatial Discretizations for Partial Differential Equations held in May 2004 at the IMA and the subsequent workshop entitled Compatible Discretizations for Partial Differential Equations held at the CMA in September 2005, were particularly important to the development of this field and this paper. We are grateful to the participants of these workshops for valuable discussions and presentations. We particularly acknowledge the help of Daniele Boffi, Snorre Christiansen and Joachim Schöberl, whose presentations at the workshops and subsequent discussions have influenced the presentations of parts of the paper. The first author is also grateful to Victor Reiner for his explanations of the representation theory used to characterize the affine-invariant spaces of polynomial differential form. We also gratefully acknowledge the support of this work by the National Science Foundation of the United States and the Norwegian Research Council. The work of the first author was supported in part by NSF grant DMS-0411388, the work of the second author by NSF grant DMS03-08347, and the work of the third author was supported by the Norwegian Research Council.

\section{REFERENCES}

S. Adams and B. Cockburn (2005), 'A mixed finite element method for elasticity in three dimensions', J. Sci. Comput. 25, 515-521.

M. Ainsworth and J. Coyle (2003), 'Hierarchic finite element bases on unstructured tetrahedral meshes', Internat. J. Numer. Methods Engrg. 58, 2103-2130.

M. Amara and J. M. Thomas (1979), 'Equilibrium finite elements for the linear elastic problem', Numer. Math. 33, 367-383.

C. Amrouche, C. Bernardi, M. Dauge and V. Girault (1998), 'Vector potentials in three-dimensional non-smooth domains', Math. Methods Appl. Sci. 21, 823864 . 
D. N. Arnold (2002), Differential complexes and numerical stability, in Proceedings of the International Congress of Mathematicians, Vol. I (Beijing, 2002), Higher Education Press, Beijing, pp. 137-157.

D. N. Arnold and R. S. Falk (1988), 'A new mixed formulation for elasticity', Numer. Math. 53, 13-30.

D. N. Arnold and R. Winther (2002), 'Mixed finite elements for elasticity', Numer. Math. 92, 401-419.

D. N. Arnold, P. B. Bochev, R. B. Lehoucq, R. A. Nicolaides and M. Shashkov, eds (2006a), Compatible Spatial Discretizations, Vol. 142 of The IMA Volumes in Mathematics and its Applications, Springer, Berlin.

D. N. Arnold, F. Brezzi and J. Douglas, Jr. (1984a), 'PEERS: a new mixed finite element for plane elasticity', Japan J. Appl. Math. 1, 347-367.

D. N. Arnold, J. Douglas, Jr. and C. P. Gupta (1984b), 'A family of higher order mixed finite element methods for plane elasticity', Numer. Math. 45, 1-22.

D. N. Arnold, R. S. Falk and R. Winther (1997a), 'Preconditioning discrete approximations of the Reissner-Mindlin plate model', RAIRO Modél. Math. Anal. Numér. 31, 517-557.

D. N. Arnold, R. S. Falk and R. Winther (1997b), 'Preconditioning in $H$ (div) and applications', Math. Comp. 66, 957-984.

D. N. Arnold, R. S. Falk and R. Winther (2000), 'Multigrid in $H$ (div) and $H$ (curl)', Numer. Math. 85, 197-217.

D. N. Arnold, R. S. Falk and R. Winther (2005), 'Mixed finite element methods for linear elasticity with weakly imposed symmetry', Math. Comput. submitted.

D. N. Arnold, R. S. Falk and R. Winther (2006b), Differential complexes and stability of finite element methods I: The de Rham complex, in Compatible Spatial Discretizations, Vol. 142 of The IMA Volumes in Mathematics and its Applications, Springer, Berlin, pp. 23-46.

D. N. Arnold, R. S. Falk and R. Winther (2006c), Differential complexes and stability of finite element methods II: The elasticity complex, in Compatible Spatial Discretizations, Vol. 142 of The IMA Volumes in Mathematics and its Applications, Springer, Berlin, pp. 47-68.

V. I. Arnold (1978), Mathematical Methods of Classical Mechanics, SpringerVerlag, New York.

I. Babuška and A. K. Aziz (1972), Survey lectures on the mathematical foundations of the finite element method, in The Mathematical Foundations of the Finite Element Method with Applications to Partial Differential Equations (Proc. Sympos., Univ. Maryland, Baltimore, Md., 1972), Academic Press, New York, pp. 1-359.

I. Babuška and J. Osborn (1991), Eigenvalue problems, in Handbook of Numerical Analysis, Vol. II, North-Holland, Amsterdam, pp. 641-787.

I. N. Bernštel̆n, I. M. Gel'fand and S. I. Gel'fand (1975), Differential operators on the base affine space and a study of $\mathfrak{g}$-modules, in Lie Groups and Their Representations (Proc. Summer School, Bolyai János Math. Soc., Budapest, 1971), Halsted, New York, pp. 21-64.

P. Bochev and M. D. Gunzburger (2005), 'On least-squares finite element methods for the Poisson equation and their connection to the Dirichlet and Kelvin principles', SIAM J. Numer. Anal. 43, 340-362. 
P. B. Bochev and J. M. Hyman (2006), Principles of mimetic discretizations of differential operators, in Compatible Spatial Discretizations, Vol. 142 of The IMA Volumes in Mathematics and its Applications, Springer, Berlin, pp. 89119.

D. Boffi (2000), 'Fortin operator and discrete compactness for edge elements', $\mathrm{Nu}$ mer. Math. 87, 229-246.

D. Boffi (2001), 'A note on the de Rham complex and a discrete compactness property', Appl. Math. Lett. 14, 33-38.

D. Boffi (2006), Compatible discretizations for eigenvalue problems, in Compatible Spatial Discretizations, Vol. 142 of The IMA Volumes in Mathematics and its Applications, Springer, Berlin, pp. 121-142.

D. Boffi and L. Gastaldi (2002), 'Edge finite elements for the approximation of Maxwell resolvent operator', M2AN Math. Model. Numer. Anal. 36, 293305.

D. Boffi, F. Brezzi and L. Gastaldi (1997), 'On the convergence of eigenvalues for mixed formulations', Ann. Scuola Norm. Sup. Pisa Cl. Sci. (4) 25, 131-154 (1998).

D. Boffi, F. Brezzi and L. Gastaldi (2000), 'On the problem of spurious eigenvalues in the approximation of linear elliptic problems in mixed form', Math. Comp. 69, 121-140.

A. Bossavit (1988), 'Whitney forms: A class of finite elements for three-dimensional computations in electromagnetism', IEE Trans. Mag. 135, Part A, 493-500.

R. Bott and L. W. Tu (1982), Differential Forms in Algebraic Topology, Vol. 82 of Graduate Texts in Mathematics, Springer, New York.

D. Braess and W. Hackbusch (1983), 'A new convergence proof for the multigrid method including the $V$-cycle', SIAM J. Numer. Anal. 20, 967-975.

J. H. Bramble (1993), Multigrid methods, Vol. 294 of Pitman Research Notes in Mathematics Series, Longman Scientific \& Technical, Harlow.

J. H. Bramble and J. E. Pasciak (1988), 'A preconditioning technique for indefinite systems resulting from mixed approximations of elliptic problems', Math. Comp. 50, 1-17.

J. H. Bramble and J. E. Pasciak (1997), 'Iterative techniques for time dependent Stokes problems', Comput. Math. Appl. 33, 13-30. Approximation theory and applications.

S. C. Brenner and L. R. Scott (2002), The mathematical theory of finite element methods, Vol. 15 of Texts in Applied Mathematics, second edn, Springer, New York.

F. Brezzi (1974), 'On the existence, uniqueness and approximation of saddle-point problems arising from Lagrangian multipliers', Rev. Française Automat. Informat. Recherche Opérationnelle Sér. Rouge 8, 129-151.

F. Brezzi and M. Fortin (1991), Mixed and Hybrid Finite Element Methods, Vol. 15 of Springer Series in Computational Mathematics, Springer, New York.

F. Brezzi, J. Douglas, Jr. and L. D. Marini (1985), 'Two families of mixed finite elements for second order elliptic problems', Numer. Math. 47, 217-235.

F. Brezzi, J. Douglas, Jr., R. Durán and M. Fortin (1987), 'Mixed finite elements for second order elliptic problems in three variables', Numer. Math. 51, 237-250. 
A. Čap, J. Slovák and V. Souček (2001), 'Bernstein-Gelfand-Gelfand sequences', Ann. of Math. (2) 154, 97-113.

S. H. Christiansen (2005), 'Stability of hodge decompositions of finite element spaces of differential forms in arbitrary dimensions', Preprint, Dept. of Math., University of Oslo.

P. G. Ciarlet (1978), The finite element method for elliptic problems, North-Holland Publishing Co., Amsterdam.

P. Clément (1975), 'Approximation by finite element functions using local regularization', Rev. Française Automat. Informat. Recherche Opérationnelle Sér. Rouge, RAIRO Analyse Numérique 9, 77-84.

M. Costabel (1991), 'A coercive bilinear form for Maxwell's equations', J. Math. Anal. Appl. 157, 527-541.

M. Desbrun, A. N. Hirani, M. Leok and J. E. Marsden (2005), 'Discrete exterior calculus'. available from arXiv.org/math.DG/0508341.

J. Douglas, Jr. and J. E. Roberts (1985), 'Global estimates for mixed methods for second order elliptic equations', Math. Comp. 44, 39-52.

M. Dryja and O. B. Widlund (1995), 'Schwarz methods of Neumann-Neumann type for three-dimensional elliptic finite element problems', Comm. Pure Appl. Math. 48, 121-155.

M. Eastwood (2000), 'A complex from linear elasticity', Rend. Circ. Mat. Palermo (2) Suppl. (63), 23-29.

R. S. Falk and J. E. Osborn (1980), 'Error estimates for mixed methods', RAIRO Anal. Numér. 14, 249-277.

M. Farhloul and M. Fortin (1997), 'Dual hybrid methods for the elasticity and the Stokes problems: a unified approach', Numer. Math. 76, 419-440.

H. Federer (1969), Geometric Measure Theory, Vol. 153 of Die Grundlehren der mathematischen Wissenschaften, Springer, New York.

G. J. Fix, M. D. Gunzburger and R. A. Nicolaides (1981), 'On mixed finite element methods for first-order elliptic systems', Numer. Math. 37, 29-48.

B. M. Fraeijs de Veubeke (1965), Displacement and equilibrium models in the finite element method, in Stress Analysis (O. C. Zienkiewicz and G. S. Holister, eds), Wiley, New York, pp. 145-197.

W. Fulton and J. Harris (1991), Representation Theory, Vol. 129 of Graduate Texts in Mathematics, Springer, New York.

M. P. Gaffney (1951), 'The harmonic operator for exterior differential forms', Proc. Nat. Acad. Sci. USA 37, 48-50.

V. Girault and P.-A. Raviart (1986), Finite element methods for Navier-Stokes equations, Vol. 5 of Springer Series in Computational Mathematics, Springer, Berlin.

J. Gopalakrishnan, L. E. García-Castillo and L. F. Demkowicz (2005), 'Nédélec spaces in affine coordinates', Comput. Math. Appl. 49, 1285-1294.

V. W. Guillemin and S. Sternberg (1999), Supersymmetry and Equivariant de Rham Theory, Mathematics Past and Present, Springer, Berlin.

W. Hackbusch (1994), Iterative Solution of Large Sparse Systems of Equations, Vol. 95 of Applied Mathematical Sciences, Springer, New York. Translated and revised from the 1991 German original. 
P. J. Hilton and U. Stammbach (1997), A Course in Homological Algebra, Vol. 4 of Graduate Texts in Mathematics, 2nd edn, Springer, New York.

R. Hiptmair (1997), 'Multigrid method for H(div) in three dimensions', Electron. Trans. Numer. Anal. 6, 133-152 (electronic). Special issue on Multilevel Methods (Copper Mountain, CO, 1997).

R. Hiptmair (1999a), 'Canonical construction of finite elements', Math. Comp. 68, $1325-1346$.

R. Hiptmair (1999b), 'Multigrid method for Maxwell's equations', SIAM J. Numer. Anal. 36, 204-225 (electronic).

R. Hiptmair (2001), Higher order Whitney forms, in Geometrical Methods in Computational Electromagnetics (F. Teixeira, ed.), Vol. 32 of PIER, EMW Publishing, Cambridge, MA, pp. 271-299.

R. Hiptmair (2002), Finite elements in computational electromagnetism, in Acta Numerica, vol. 11, Cambridge University Press, Cambridge, pp. 237-339.

R. Hiptmair and A. Toselli (2000), Overlapping and multilevel Schwarz methods for vector valued elliptic problems in three dimensions, in Parallel Solution of Partial Differential Equations (Minneapolis, MN, 1997), Vol. 120 of IMA Vol. Math. Appl., Springer, New York, pp. 181-208.

K. Jänich (2001), Vector Analysis, Undergraduate Texts in Mathematics, Springer, New York. Translated from the second German (1993) edition by Leslie Kay.

C. Johnson and B. Mercier (1978), 'Some equilibrium finite element methods for two-dimensional elasticity problems', Numer. Math. 30, 103-116.

T. Kato (1995), Perturbation Theory for Linear Operators, Classics in Mathematics, Springer, Berlin. Reprint of the 1980 edition.

A. Klawonn (1998), 'An optimal preconditioner for a class of saddle point problems with a penalty term', SIAM J. Sci. Comput. 19, 540-552 (electronic).

S. Lang (1995), Differential and Riemannian manifolds, Vol. 160 of Graduate Texts in Mathematics, Springer, New York.

J.-L. Loday (1992), Cyclic Homology, Vol. 301 of Grundlehren der Mathematischen Wissenschaften, Springer, Berlin.

B. Mercier, J. Osborn, J. Rappaz and P.-A. Raviart (1981), 'Eigenvalue approximation by mixed and hybrid methods', Math. Comp. 36, 427-453.

D. Mitrea, M. Mitrea and M. Taylor (2001), 'Layer potentials, the Hodge Laplacian, and global boundary problems in nonsmooth Riemannian manifolds', Mem. Amer. Math. Soc. 150, x+120.

M. Mitrea (2001), 'Dirichlet integrals and Gaffney-Friedrichs inequalities in convex domains', Forum Math. 13, 531-567.

P. Monk (1992), 'A finite element method for approximating the time-harmonic Maxwell equations', Numer. Math. 63, 243-261.

P. Monk (2003a), Finite element methods for Maxwell's equations, Numerical Mathematics and Scientific Computation, Oxford University Press, New York.

P. Monk (2003b), A simple proof of convergence for an edge element discretization of Maxwell's equations, in Computational electromagnetics (Kiel, 2001), Vol. 28 of Lecture Notes in Computer Science and Engineering, Springer, Berlin, pp. 127-141. 
P. Monk and L. Demkowicz (2001), 'Discrete compactness and the approximation of Maxwell's equations in $\mathbb{R}^{3}$, Math. Comp. 70, 507-523.

M. E. Morley (1989), 'A family of mixed finite elements for linear elasticity', Numer. Math. 55, 633-666.

J.-C. Nédélec (1980), 'Mixed finite elements in $\mathbf{R}^{3}$ ', Numer. Math. 35, 315-341.

J.-C. Nédélec (1986), 'A new family of mixed finite elements in $\mathbf{R}^{3}$, Numer. Math. 50, 57-81.

R. A. Nicolaides and K. A. Trapp (2006), Covolume discretization of differential forms, in Compatible Spatial Discretizations, Vol. 142 of The IMA Volumes in Mathematics and its Applications, Springer, Berlin, pp. 89-119.

J. E. Osborn (1979), Eigenvalue approximation by mixed methods, in Advances in Computer Methods for Partial Differential Equations, III (Proc. Third IMACS Internat. Sympos., Lehigh Univ., Bethlehem, Pa., 1979), IMACS, New Brunswick, NJ, pp. 158-161.

R. Picard (1984), 'An elementary proof for a compact imbedding result in generalized electromagnetic theory', Math. Z. 187, 151-164.

P.-A. Raviart and J. M. Thomas (1977), A mixed finite element method for 2nd order elliptic problems, in Mathematical aspects of finite element methods (Proc. Conf., Consiglio Naz. delle Ricerche (C.N.R.), Rome, 1975), Vol. 606 of Lecture Notes in Mathematics, Springer, Berlin, pp. 292-315.

T. Rusten and R. Winther (1992), 'A preconditioned iterative method for saddlepoint problems', SIAM J. Matrix Anal. Appl. 13, 887-904. Special issue on Iterative methods in numerical linear algebra (Copper Mountain, CO, 1990).

J. Schöberl (2005), 'A posteriori error estimates for Maxwell equations', RICAM Report No. 2005-10.

D. Silvester and A. Wathen (1994), 'Fast iterative solution of stabilised Stokes systems. II. Using general block preconditioners', SIAM J. Numer. Anal. 31, 1352-1367.

B. F. Smith, P. E. Bjørstad and W. D. Gropp (1996), Domain Decomposition: Parallel Multilevel Methods for Elliptic Partial Differential Equations, Cambridge University Press, Cambridge.

E. Stein and R. Rolfes (1990), 'Mechanical conditions for stability and optimal convergence of mixed finite elements for linear plane elasticity', Comput. Methods Appl. Mech. Engrg. 84, 77-95.

R. Stenberg (1986), 'On the construction of optimal mixed finite element methods for the linear elasticity problem', Numer. Math. 48, 447-462.

R. Stenberg (1988a), 'A family of mixed finite elements for the elasticity problem', Numer. Math. 53, 513-538.

R. Stenberg (1988b), Two low-order mixed methods for the elasticity problem, in The Mathematics of Finite Elements and Applications, VI (Uxbridge, 1987), Academic Press, London, pp. 271-280.

M. E. Taylor (1996), Partial Differential Equations. I: Basic Theory, Vol. 115 of Applied Mathematical Sciences, Springer, New York.

P. S. Vassilevski and J. P. Wang (1992), 'Multilevel iterative methods for mixed finite element discretizations of elliptic problems', Numer. Math. 63, 503-520.

V. B. Watwood, Jr. and B. J. Hartz (1968), 'An equilibrium stress field model for 
finite element solution of two-dimensional elastostatic problems', Internat. J. Solids Structures 4, 857-873.

J. P. Webb (1999), 'Hierarchal vector basis functions of arbitrary order for triangular and tetrahedral finite elements', IEEE Trans. Antennas and Propagation 47, 1244-1253.

H. Whitney (1957), Geometric Integration Theory, Princeton University Press, Princeton, NJ.

J. Xu (1992), 'Iterative methods by space decomposition and subspace correction', SIAM Rev. 34, 581-613. 